\title{
Offering a helping hand
}

Getting a grip on needs and preferences of stroke patients regarding soft-robotic technology supporting hand function

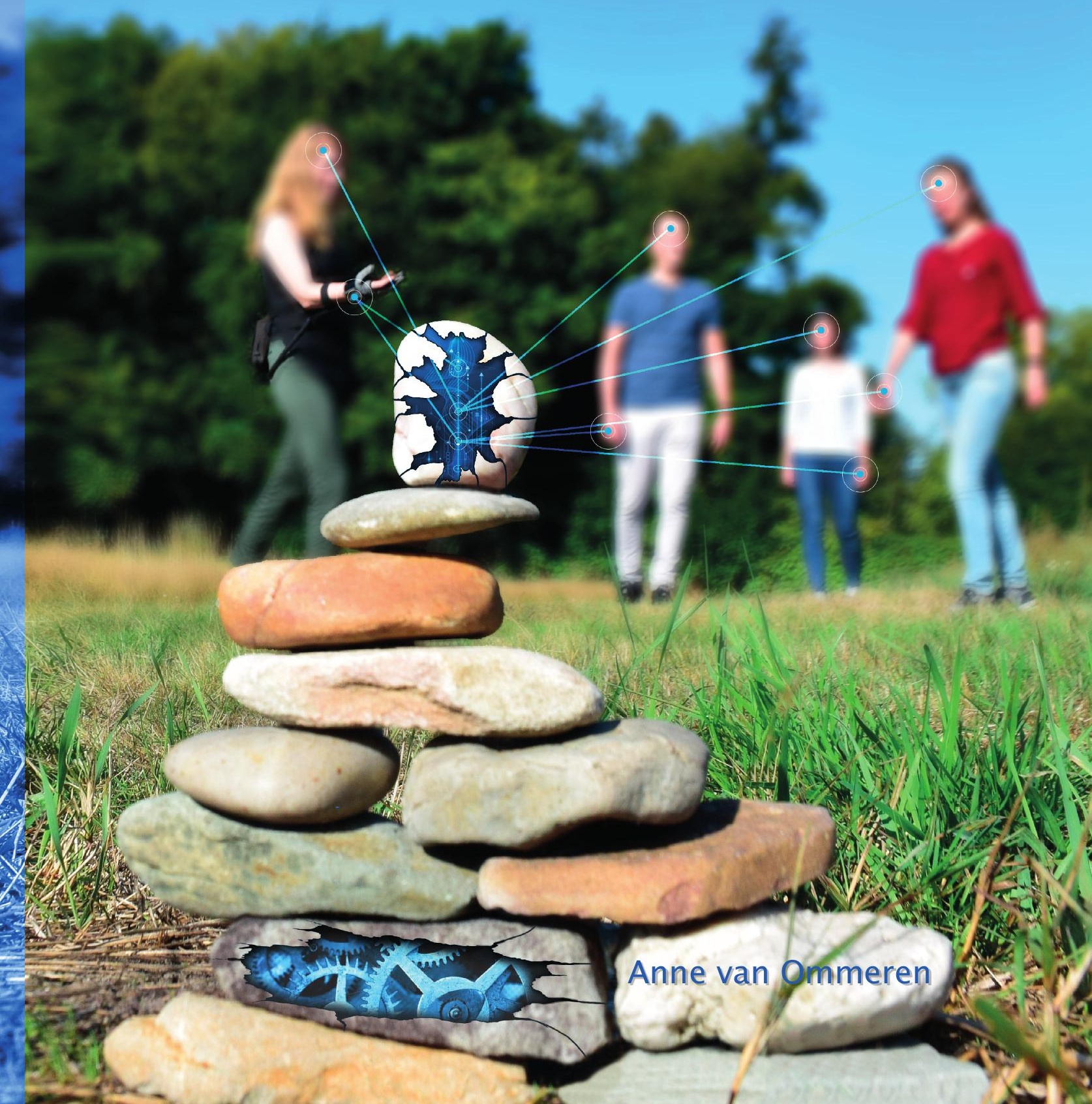





\section{OFFERING A HELPING HAND}

GETTING A GRIP ON NEEDS AND PREFERENCES OF STROKE PATIENTS REGARDING SOFT-ROBOTIC TECHNOLOGY SUPPORTING

HAND FUNCTION

Anne Lotte van Ommeren 


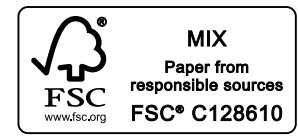

Part of the work in this thesis was supported by the European Union's Horizon 2020 research and innovation programme under grant agreement No 644000. The soft-robotic glove used in this thesis was provided by Bioservo Technologies AB, Sweden.

The publication of this thesis was generously supported by:
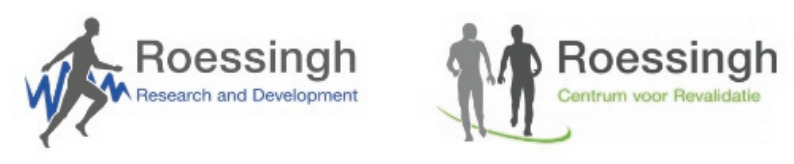

Cover design

Jos Spoelstra

Printed by Ipskamp, Enschede, the Netherlands

ISBN 978-90-365-4835-9

(C) Anne van Ommeren, Enschede, the Netherlands, 2019 All rights reserved. No part of this book may be reproduced, stored in a retrieval system, or transmitted, in any form or by any means, electronic, mechanical, photocopying, recording, or otherwise, without prior written permission of the holder of the copyright. 


\title{
OFFERING A HELPING HAND
}

\section{GETTING A GRIP ON NEEDS AND PREFERENCES OF STROKE \\ PATIENTS REGARDING SOFT-ROBOTIC TECHNOLOGY SUPPORTING \\ HAND FUNCTION}

\begin{abstract}
PROEFSCHRIFT
ter verkrijging van

de graad van doctor aan de Universiteit Twente,

op gezag van de rector magnificus

prof. dr. T.T.M. Palstra

volgens besluit van het College voor Promoties

in het openbaar te verdedigen

op donderdag 31 oktober 2019 om 14:45 uur
\end{abstract}

door

Anne Lotte van Ommeren

geboren op 5 september 1991

te Enschede 
Dit proefschrift is goedgekeurd door:

Promotoren Prof. dr. J.S. Rietman

Prof. dr. ir. P.H. Veltink

Co-promotor Dr. G.B. Prange-Lasonder 


\section{Promotiecommissie}

\section{Voorzitter}

Prof. dr. G.P.M.R. Dewulf

Universiteit Twente

\section{Promotoren}

Prof. dr. J.S. Rietman

Universiteit Twente

Prof. dr. ir. P.H. Veltink

Universiteit Twente

\section{Co-promotor}

dr. G.B. Prange-Lasonder

Universiteit Twente

\section{Leden}

Prof. dr. ir. H.F.J.M. Koopman

Universiteit Twente

Prof. dr. ir. H.J. Hermens

Universiteit Twente

Prof. dr. J.M.A. Visser-Meily

Universiteit Utrecht

Prof. dr. J.H. Burridge

University of Southampton

Prof. dr. Z. Matjačić

University of Ljubljana 



\section{Table of contents}

Chapter 1 General introduction

Chapter 2 Assistive technology for the upper extremities after stroke: systematic review of users' needs

Chapter 3 Identification of user requirements for assistive technology for support of upper limb daily life interactions from stroke patients

Chapter 4 Feasibility and user experience of control modalities for a gripsupporting soft-robotic glove: comparing force, muscle activity, voice and wink control with stroke patients

Chapter 5 Quantifying upper extremity performance with and without assistance of a soft-robotic glove in elderly patients: a kinematic analysis

Chapter 6 Quantifying the direct influence of a soft-robotic glove on task performance of the affected upper extremity after stroke

Chapter 7 The effect of prolonged use of a wearable soft-robotic glove post stroke - a proof-of-principle

Chapter 8 Detection of the intention to grasp during reach movements

Chapter 9 Detection of the intention to grasp during reaching in stroke using inertial sensing

Chapter 10 General discussion

\&

References

Summary

Samenvatting

Dankwoord

Author contributions

Progress range 



\section{General introduction Chapter 1}

\section{Stroke}

Stroke is one of the primary causes of permanent disability [1, 2]. It is estimated that the number of people older than 65 in the Netherlands will almost double, from 2.4 million to 4.5 million, between 2008 and 2040 [3]. Since the risk of stroke increases substantially with age, the incidence of stroke is likely to rise in the coming decades. Besides the rising trend of stroke incidence, the number of stroke related deaths reduced from 153 per 100.000 inhabitants in 2000 to 110 per 100.000 in 2016 [4]. The decrease in stroke mortality rate is likely due to improvements in acute and long-term care [5]. In addition to the improvements in care, the number of hospitalizations caused by stroke increased substantially in the last decades [4]. In the future, great strain will be placed on national healthcare services as a result of the rising trend of stroke incidence and hospitalizations [6].

Stroke is caused by an interrupted blood flow in the brain, either of hemorrhagic or ischemic cause, which leads to a disturbed generation and integration of neural commands. Resulting impairments of the interrupted blood flow may vary depending on the area in which the interruption manifests. Cognitive, emotional and sensory disorders are commonly experienced post stroke, yet upper extremity weakness or hemiparesis are the most common impairments [7]. Recovery of upper extremity function is one of the primary goals of rehabilitation. To stimulate motor recovery after stroke, several studies have shown that it is crucial to focus on functional activities with an active contribution of the stroke patient [8-10].

\section{Functional problems in daily life at home}

Yet after conventional rehabilitation, only $10-15 \%$ of the stroke patients have regained complete functional use of the upper limb during daily life activities (ADL) six months post stroke [11]. One year post stroke, an estimated $40 \%$ of the patients still needs assistance in ADL [8]. Due to the loss of functional use of the hand and arm, stroke patients experience difficulties in personal care activities, especially those involving handling of objects such as drinking, eating, opening buttons on clothing, and writing independently. Functional independence and the quality of life of stroke patients are often affected by this loss in dexterity and corresponding limitations in $\operatorname{ADL}[12,13]$. As a consequence of having diminished upper extremity function, stroke patients tend to avoid involvement of their affected side in ADL. Moreover, independence might be limited even more due to prolonged non-use of the hand [14]. 


\section{Robotic devices for the hand}

Use of the affected hand can be promoted by intensive use during repetitive, task-specific tasks or therapy based on the essential principles of motor relearning [15-17]. Over the last decades, attention is given to the development of robotic training devices for the UE, initially focused on the proximal part of the UE but the number of reported devices for hand and/ or wrist movements is increasing $[18,19]$. Although promising results of robot-assisted hand rehabilitation are reported that resulted in reduction of motor impairment of the hand and arm, robotic devices designed for upper limb therapy fail to transfer such gains into higher levels of functional independence [20]. One way for stroke patients to increase their functional independence is by being directly supported by technology during ADL in their homes. Currently, orthoses fixating a particular joint in a certain position (e.g., the wrist can be placed in a more functional orientation using a wrist extension splint) and ergonomic tools that can assist people during a specific task are used to regain some functionality during ADL [21]. More sophisticated fully robotic devices are also available for people with very severe limitations which allow more functionality [22]. While simple tools are usually easy to use and small, robotic systems often consist of heavy, bulky, complex and expensive pieces of equipment. In theory, use of assistive technology can reduce personal assistance or (in)formal care by 30 $41 \%[22,23]$. In this thesis, assistive technology is defined as electrical or mechanical devices designed to help people recover upper extremity function by offering direct assistance.

Although AT have the potential to increase independency of stroke patients by reducing informal and formal care, in practice it appears to be difficult to apply such robotic devices in ADL of stroke patients despite the growing number of assistive technology developed for the hand $[19,24]$. The mismatch between the needs and preferences of the end-users and their environment regarding the design of the device combined with the complexity of most robotic devices is believed to be the main reason for the low uptake of assistive technology. For assistive technology to be used during ADL it is of great importance not to interfere with the social and physical interactions encountered in everyday life of stroke patients. The importance of an assistive technology to be easy and intuitive to use, portable, easy to set up and lightweight is, among other factors, highlighted by end-users [5, 24-30]. However, no comprehensive set of user requirements is yet established.

\section{User-centered design}

The consideration of user preferences and wishes is crucial for the potential acceptance, or abandonment, of developed assistive technology [31]. Although it seems that the development of upper limb assistive technology still tends to be technology driven [32], the concept of user involvement throughout the design process is known for years [33]. Development according 
to a user-centered design, in which end-users are involved in the design, is believed to be an effective means to meet the preferences and wishes of the end-users [34].

Placing the user at the center of the design process can be achieved by numerous methods depending on the stage of development (Figure 1.1). Early in the design cycle, information via interviews, questionnaires, focus groups and on-site observations can be obtained. From the mid-point on, when a prototype is available, walkthroughs, simulations and usability testing are informative methods to gain additional information about user expectations, usability and user satisfaction.[34] Although applying a user-centered design can be costly and time consuming, the approach can result in development of more effective, efficient and safe products with higher chances of actual uptake of the device in daily life [34-36].

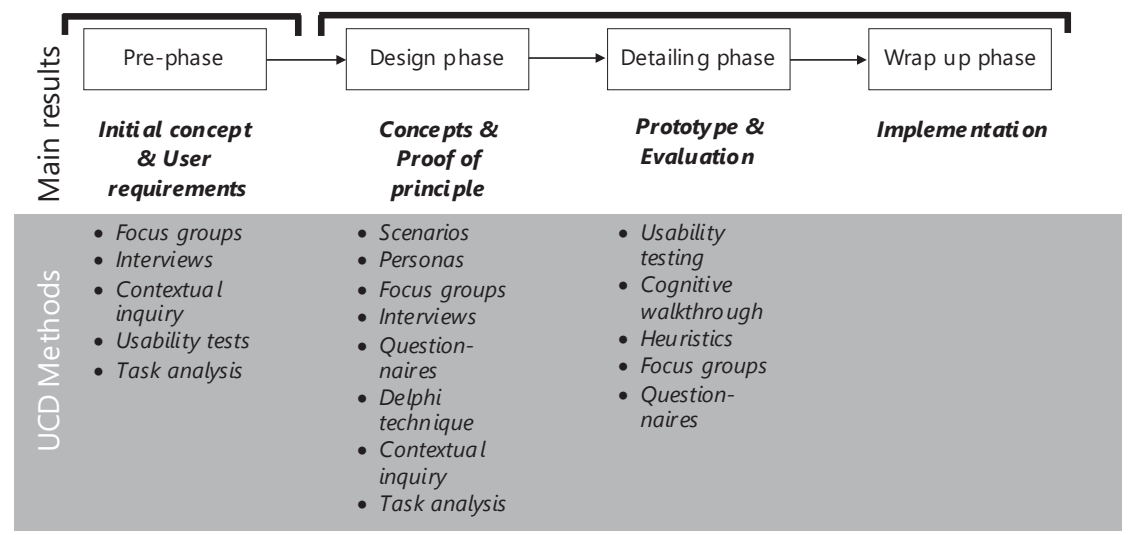

Figure 1.1 Typical phases of a design project (adapted from Eger et al.[37] and Martin et al.[38])

\section{eNHANCE}

A new device for home-use that supports the arm, wrist and hand was developed in the eNHANCE project (Figure 1.2). This project aimed to enable stroke patients in performing daily life interactions involving their affected hand and arm while maximizing their own contribution. The system was developed using a user-centered iterative cycle design methodology, initially starting with the identification of user requirements. Since there is a huge variation in experienced symptoms, stroke patients in the study of Elnady et al.[39] expressed the need for the design of an AT to be targeted at the individual. While the importance of personalization of treatment has been recognized in rehabilitation, is the design of AT for support of the upper limb of stroke patients not yet targeted at the individual user. Personalization could simply be reached by hardware adaptations such as offering different sizes, but personalization of modalities to control a device could make the device accessible for a wider group and will likely increase its usability. Currently, active devices for the hand are controlled by only one 
modality [40], which was chosen for without the involvement of end-users. Therefore, from the start of the eNHANCE project on, focus was placed on key concepts such as personalization, and sensing modalities to control the device. For pragmatic reasons we chose to focus on the hand support system in the thesis.

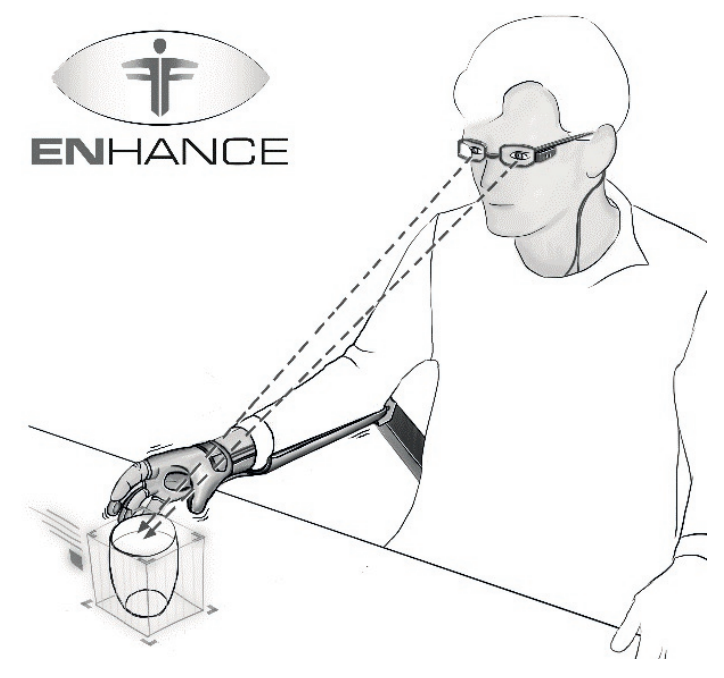

Figure 1.2 Schematic presentation of the eNHANCE system

\section{The ironHand system}

The ironHand, a wearable soft-robotic glove (Figure 1.3), was developed, and evaluated with elderly, in previous projects according to a user-centered design in order to support grip strength during daily life activities of people experiencing hand function problems. The ironHand system consists of a three-fingered glove (ca. 85 grams) and a control unit (ca. 600 grams) containing the embedded software to control the force needed to support grip strength, and the batteries. The glove and the control unit are connected via a cord in which the electrical cables for the sensors and the artificial tendons are located. Grip strength of the hand is supported by the glove via the thumb, middle and ring finger. Pressure sensors (Interlink Electronics) are located at the fingertips of the glove. The level of support is regulated by a tendon-driven mechanism which receives input from the pressure sensors. The glove is available in three different sizes to make sure that the glove tightly fits the hand of the user. 


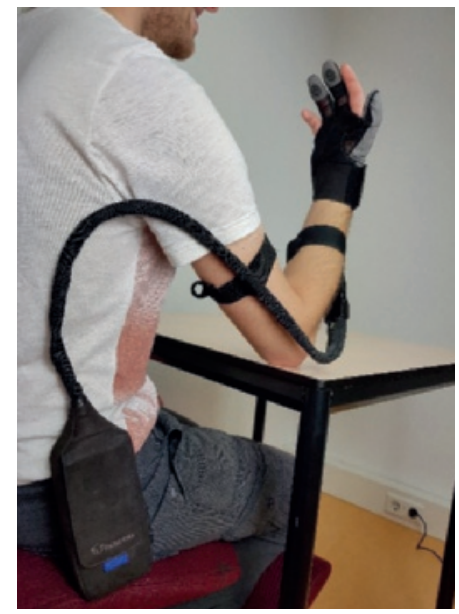

Figure 1.3 The ironHand system

\section{General aim and research questions}

The potential acceptance of AT depends on whether the user requirements (prior to use) are met, but probably also depends on usability and effectiveness of a device [19, 24]. To date, there is no comprehensive overview of what the stroke patients' needs and preferences are. Besides, information on usability and effect (both immediately and after prolonged use) of the ironHand system on movement execution and task performance in stroke is not present either. As a next step in the design of AT for stroke, this information about user requirements, usability and effectiveness could be used in the future to design AT with sensing and control that better meet the needs and preferences of the end-users.

This thesis aims to increase our understanding on user requirements on AT, and on the usability and effectiveness of the ironHand system, to contribute to the development of assistive technologies to support the hand of stroke patients during ADL at home that meet the needs and preferences of the end-user.

Based on the general objective, this thesis aims to answer the following research questions:

1. What defines the user preferences concerning AT designed for the upper limb of stroke patients?

2. Does a wearable soft-robotic glove improve task performance directly?

3. Does prolonged, unsupervised, use of a wearable soft-robotic glove during ADL at home improve task performance of stroke patients?

4. Considering the limitations of pressure sensing as control input for the device, could the control of the grip-supporting glove be improved? 


\section{Outline}

User perspectives on assistive technology for the upper limb post stroke are presented in this thesis. Prior to development, existing literature on end-user perspectives are combined into a theoretical framework in order to gain more insight into the facilitators and barriers that can bring the design of assistive technology for the upper limb to higher levels of user satisfaction (chapter 2). To supplement the information retrieved from literature, we aimed to identify technology specific end-users' requirements for upper extremity assistive technology to support daily life interactions in chapter 3 . To evaluate user preferences on the mechanism to control the wearable soft-robotic glove, we assessed and compared user perspectives on, and feasibility of, force, muscle activity, voice and wink controlled grasping in chapter 4 .

In the second part of this thesis, the direct effect of the ironHand system on movement execution is explored in both elderly (chapter 5) and stroke patients (chapter 6). After assessing the direct effect, the potential, and effect, of the assistive glove while being used for a prolonged time unsupervised at home was assessed as a next step towards use of a wearable assistive device in the home environment (chapter 7). In this proof-of-principle, the direct effect, as well as its assistive and therapeutic potential on hand function, were explored.

Based on the findings of the preceding chapters that focused on the user requirement identification, usability and effectiveness of the ironHand, arose the idea to research the possibility to detect the intention to grasp earlier than the pressure sensors of the current version of the ironHand system. This is investigated in healthy subjects (chapter 8) and subsequently in stroke patients (chapter $\mathbf{9}$ ).

Finally, in chapter 10, the main findings from preceding chapters, and conclusions of this thesis are discussed, along with suggestions for clinical implications and future research. 



\title{
Assistive technology for the upper extremities after stroke: systematic review of users' needs
}

Chapter 2

\begin{abstract}
Background: Technical innovations have the potential to compensate for loss of upper-limb motor functions after stroke. However, the majority of the designs do not completely meet the needs and preferences of the end users. User-centered design methods have shown that the attention to user perspectives during development of assistive technology (AT) leads to devices that better suit the needs of the users.
\end{abstract}

Objective: To get more insight into the factors that can bring the design of AT to higher levels of satisfaction and acceptance, studies about user perspectives on AT for the upper limb after stroke are systematically reviewed.

Methods: A database search was conducted in PubMed, EMBASE, CINAHL, PsycINFO, and Scopus from inception to August 2017, supplemented with a search of reference lists. Methodological quality of the included studies was appraised. User perspectives of stroke patients, carers, and healthcare professionals were extracted. A total of 35 descriptive themes were identified, from which 5 overarching themes were derived.

Results: In total, 9 studies with information gathered from focus groups, questionnaires, and interviews were included. Barriers and enablers influencing the adoption of AT for the upper limb after stroke emerged within 5 overarching, but highly interdependent themes: (1) promoting hand and arm performance; (2) attitude toward technology; (3) decision process; (4) usability; and (5) practical applicability.

Conclusions: Expected use of an AT is facilitated when it has a clear therapeutic base (expected benefit in enhancing function), its users (patients and healthcare professionals) have a positive attitude toward technology, sufficient information about the AT is available, and usability and practical applicability have been addressed successfully in its design. The interdependency of the identified themes implies that all aspects influencing user perspectives of AT need to be considered when developing AT to enhance its chance of acceptance. The importance of each factor may vary depending on personal factors and the use context, either at home as an assistive aid or for rehabilitation at a clinic. 


\section{Introduction}

Stroke is one of the main causes of permanent disability [1, 2]. The risk of stroke increases substantially with age as the stroke incidence almost doubles with each decade after the age of 45 years [4]. As a result of the aging population, the number of people older than 65 years in the Netherlands is estimated to almost double (from 2.4 million-4.5 million) between 2008 and 2040 [3]. On the basis of the demographic trends alone, the incidence of stroke will rise in the coming decades. Besides, the number of deaths because of stroke decreased from 153 per 100,000 inhabitants in 2000 to 110 per 100,000 in 2016 [4], and the number of hospitalizations caused by stroke increased from 370 per 100,000 inhabitants in the year 2000 to 482 per 100,000 inhabitants in 2016 [4]. In addition, the stroke mortality rate is likely to decrease because of improvements in acute and long-term care [5]. The rising trend of stroke incidence and hospitalizations will place great strain on national healthcare services in the future [6].

The cause of stroke is an interrupted blood flow in the brain, either of hemorrhagic or ischemic cause, leading to disturbed generation and integration of neural commands. Depending on the area in which the interruption manifests, resulting impairments vary. Cognitive, emotional, and sensory disorders are often present after a first-time stroke; however, upper extremity weakness or hemiparesis are the most common impairments [7]. With regard to the arm, only $10 \%$ to $15 \%$ of stroke patients regain complete functional use during activities of daily living (ADL) within 6 months after stroke, and approximately, another $40 \%$ will regain some dexterity in the paretic arm [11]. Recovery of upper extremity function is one of the primary goals of rehabilitation programs. About $40 \%$ of occupational therapy is directly targeted at improving ADL [41]. Several studies have shown that focusing on functional activities, with active contribution of the stroke patient, is vital in stimulating motor recovery after stroke [8-10]. Loss of functional use of the hand and arm causes severe difficulties in personal care activities, especially those involving handling of objects. This limits the independence of stroke patients and significantly reduces their quality of life $[12,13]$. By the end of the first year post stroke, an estimated $40 \%$ of stroke patients still need assistance in ADL [8].

Technical innovations, such as assistive technology (AT), provide the opportunity to compensate for loss of motor function by supporting the upper limb during the execution of ADL $[12,26]$. The definition of ATs used in this study is based on the definition proposed by Demain et al.[25] and Hughes et al.[5]. Assistive technology is defined as "Electrical or mechanical devices designed to help people recover movement by offering direct assistance to the movement of the upper extremity." ATs have great potential to assist in promoting intensive use of the arm and hand, without any increase in clinical contact time in the case of a therapeutic application or help from formal or informal carers in case of assistive application. AT can increase the 
amount of motivational activities that stroke patients perform, whether it be hobby or gaming activities they enjoy or work and ADL-related tasks that might help them regain a sense of independence. AT can be used both inside and outside the clinic $[5,42]$. Remarkably, only $25 \%$ of the robotic devices for upper extremity rehabilitation have been tested clinically within the stroke population [19], suggesting limited implementation of robotic devices in practice [24]. The complexity of robotic devices and a mismatch between the needs and preferences of the end users and their environment regarding the design of the device are believed to be the main reasons for this low implementation rate $[19,24]$. This assumption is also expected to be applicable to AT in a more general sense.

User-centered design (UCD) methods have shown that including user perspectives during the design of AT enables development of devices that better suit the needs of the users [34]. The rationale for user involvement during the design process is to design a device that will be usable, comfortable, understandable, and, ultimately, acceptable for the users [43]. Currently, the design of robotic technology for stroke rehabilitation tends to be technology-driven [32]. Although an extensive list of existing technical solutions for physical therapy of the upper limb has been provided [12], few are clinically tested [19]. When AT was tested clinically, devices that were developed according to UCD showed acceptable to promising usability scores, although room for improvement was left, mainly with regard to usability aspects $[44,45]$. This supports the importance of taking the perspectives of the end users into account during the design and development of AT.

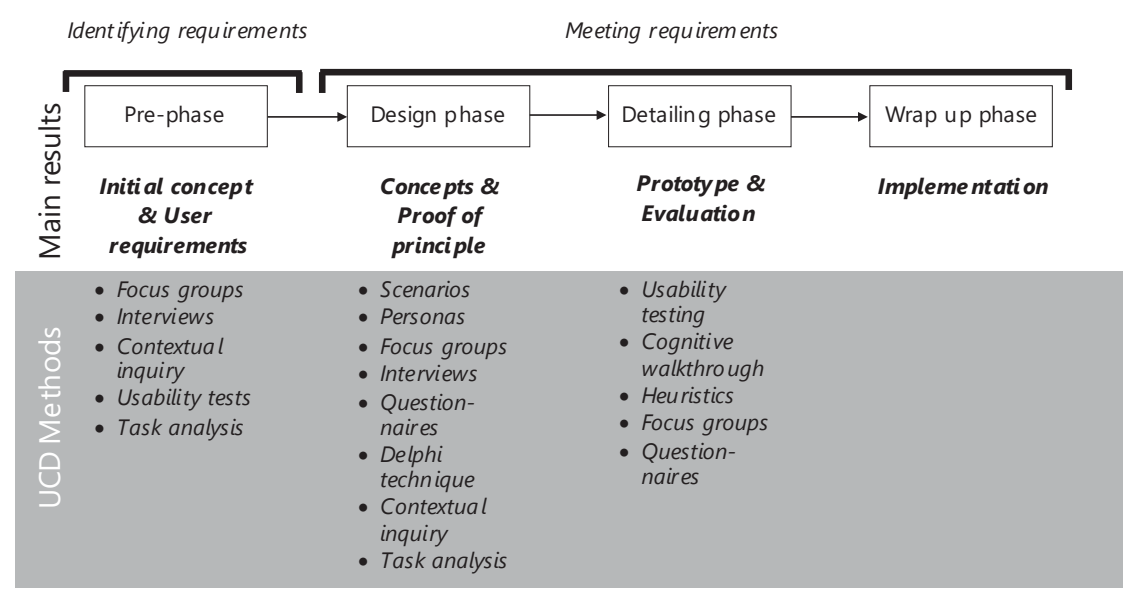

Figure 2.1 Typical phases of a design project. UCD: user-centered design. 
There is a clear need to bring assistive device design to higher levels of acceptance. Ideally, design projects should start with addressing user needs by collecting information about the target population through focus groups, interviews, questionnaires, or observation studies (Figure 2.1, adapted from Eger et al.[37] and Martin et al.[38]). Although some studies reported collection of needs and preferences of end users at the start of the design project [5, 24-30, 46], the questions asked to gather this information were often too generic.

This study, therefore, systematically reviews existing literature about user perspectives on AT for the upper extremity after stroke. The resulting insights could aid future developers in quickly determining essential user requirements that need to be addressed during the design of AT for the upper extremity after stroke to enhance its chances of acceptance by the users. The insights in this study can thus be used as a starting point for the first phase of AT development, from which developers can proceed to gather more in-depth information from their own use research, specific to their application and intended use. In the later stages of development, it remains important to involve users and incorporate UCD methods (Figure 2.1) to ensure the device will indeed meet the identified user requirements.

\section{Methods}

\section{Literature Search}

An electronic database search was conducted in PubMed, Scopus, CINAHL, Embase, and PsycINFO from inception to August 2017. The search strategy used in all these databases was a combination of the following keywords and related terms:

- Assistive technology, self-help devices, and assistive devices

- Rehabilitation robot, training devices, upper extremity rehabilitation equipment

- Robotics

- Upper extremity

- Cerebrovascular accident (CVA) and stroke

- User requirements, a priori user perspectives, and patient preferences

Reference lists of potentially relevant papers were scanned to supplement the computerized search results. Furthermore, an internet search (Google Scholar) was performed with regard to factors that affect the use of upper extremity assistive devices in the rehabilitation setting and at home.

\section{Study Selection}

The following criteria were used for the inclusion of studies: (1) studies involving qualitative or quantitative research into user perspectives; (2) involvement of stroke patients with upper extremity limitations, carers, or healthcare professionals (HCPs) of stroke patients; (3) studies 
concerning upper extremity AT; (4) studies written in English; and (5) published, full-length, and peer-reviewed papers. The definition of assistive technologies used in this review is "Electrical or mechanical devices designed to help people recover movement by offering direct assistance to the movement of the upper extremity," without distinguishing between devices designed for therapeutic purposes or home use. The included studies needed to comply with all the inclusion criteria. Thus, case studies and studies including user perspectives with regard to a product that will be designed for one specific task were excluded. Moreover, studies evaluating a prototype or product were excluded. After the duplicate citations had been excluded, 2 reviewers ( $A O$ and GP) screened titles and abstracts. Full text papers were read and summarized independently by 2 reviewers (AO and LS) and discussed subsequently. $A$ final list of papers to be included was created after consensus was reached. A third reviewer could be consulted if there was disagreement between the 2 principal reviewers (JB in case of titles and abstracts and GP in case of full papers).

\section{Methodological Research Quality Assessment}

The Critical Appraisal Skills Program (CASP) checklist was used to appraise the methodological quality of the included studies as it can guide the evaluation of a wide range of methodologies $[47,48]$. This methodological assessment tool, endorsed by the Cochrane Collaboration, contains 10 items on aims, research design and methodology, participant selection and ethics, data collection and analysis, and the statement of findings, each of which was scored as positive (yes), negative (no), or unclear (cannot tell). Each positive score received 1 point and each negative or unclear score received 0 points. Thus, the maximum possible methodological quality score was 10 . Studies were not excluded based on the CASP score; rather, the CASP score was used as reference to serve as a guide during interpretation of the results.

\section{Data Extraction}

The content of the included studies was analyzed using a structured approach, scanning for information (where available) regarding descriptive features of the population involved and the type of AT and its purpose. Subsequently, factors related to the successful or unsuccessful use of AT were collected and used as input for the analysis of this review. Therefore, information and quotations from participants under the headings results or findings were retrieved from each study.

\section{Data Synthesis}

Meta-synthesis attempts to integrate results from interrelated qualitative studies. In contrast to meta-analysis, meta-synthesis has an interpretive rather than aggregating intent [49]. In this study, the data synthesis was based on the 3-phase process from Thomas and Harden's thematic synthesis [50]. In the first phase of data synthesis, line-by-line coding of the findings 
of primary studies was performed by 2 reviewers (AO and LS). Second, descriptive themes based on the expressions found in the first phase were developed. Examples of those descriptive themes can be found in Table 2.1. Third, the descriptive themes were presented to a multidisciplinary team experienced in the field of rehabilitation technology to develop consensus-based analytical overarching themes that encompass all descriptive themes. The team consisted of a human movement scientist, electrical engineer, industrial design engineer, biomedical engineer, and a psychologist, of which the majority had not been involved in previous phases of this study. Each study was read several times by 2 reviewers (AO and LS) to ensure that all the perspectives of the participants were captured.

Table 2.1 Derivation and content of descriptive and analytical themes.

\begin{tabular}{lll}
\hline $\begin{array}{l}\text { Overarching theme and corresponding } \\
\text { descriptive factors }\end{array}$ & Derived from & $\begin{array}{l}\text { Example expressions and } \\
\text { citations }\end{array}$ \\
\hline
\end{tabular}

\section{Theme 1 Promoting hand and arm performance}

- Goal-oriented exercises

- Repetition

- Intensity

- Active contribution

- Focus on hand and arm

\section{Theme 2 Attitude toward technology}

- Motivation

- $\quad$ Familiarity and affinity with technology

- Digital security and privacy

[24, 26-30, 46]

[25, 28-30]

$[25,29,46]$

$[24,26,28,30]$

$[5,25,27]$
"Therapists stated that training should be oriented at a patient's goal(s) and his/her ability to accomplish these goal(s)" [29]

\begin{abstract}
"All participants believed that using home-based technology aimed at arm exercises would help them perform more arm exercises. It will motivate them to engage more in the exercise program" [27]
\end{abstract}

\section{Theme 3 Decision process}

- Knowledge

$[5,25,30]$

- Evidence-based practice

- Advice

- Time investment

- Safety aspects regulations

- $\quad$ Trust and expected usefulness

$[5,25,27,28,30]$

- Independence and self-management

$[5,25,27-29,46]$

- Money
"All patient participants were keen to self-manage. They were all actively engaged in looking for solutions to promote arm recovery and were prepared to spend time and, if necessary, money on potential solutions, including assistive technologies" [25] 
Table 2.1 Derivation and content of descriptive and analytical themes. (continued)

\begin{tabular}{|c|c|c|}
\hline $\begin{array}{l}\text { Overarching theme and corresponding } \\
\text { descriptive factors }\end{array}$ & Derived from & $\begin{array}{l}\text { Example expressions and } \\
\text { citations }\end{array}$ \\
\hline \multicolumn{3}{|l|}{ Theme 4 Usability } \\
\hline - Donning/doffing & [24-26] & "For stroke patients and \\
\hline - $\quad$ Setup & {$[5,25,27-30]$} & families, the devices needed \\
\hline - Initialization & {$[26,28-30]$} & a weak and/or contracted \\
\hline - $\quad$ Portable & {$[25,27,29,46]$} & $\begin{array}{l}\text { hand/arm ... and to be in- } \\
\text { tuitive in terms of correctly }\end{array}$ \\
\hline - $\quad$ Robustness & {$[5,27,29]$} & positioning the device" [25] \\
\hline - Instruction on exercises & {$[29,30]$} & \\
\hline - $\quad$ Comfort & {$[5,24,26]$} & \\
\hline - $\quad$ Lightweight & {$[24,26]$} & \\
\hline - $\quad$ Ease of use & {$[5,25-30]$} & \\
\hline - $\quad$ Compliant & $\begin{array}{l}{[24,25,27,28,} \\
30,46]\end{array}$ & \\
\hline - $\quad$ Adjustment to patient & {$[24,25,28,29]$} & \\
\hline - $\quad$ Technical support & [27] & \\
\hline - Maintenance & {$[25,27]$} & \\
\hline \multicolumn{3}{|l|}{ Theme 5 Applicability in practice } \\
\hline - $\quad$ Monitoring & {$[26,27,29,46]$} & "Hardware and software \\
\hline - $\quad$ Feedback & {$[25,26,28,29,46]$} & $\begin{array}{l}\text { design of technology should } \\
\text { facilitate adaptation to in- }\end{array}$ \\
\hline - Wrongly executed movements & {$[29,46]$} & dividual stroke patients or \\
\hline - Fatigue and overtraining & {$[46]$} & $\begin{array}{l}\text { patient target groups and } \\
\text { to patient progression over }\end{array}$ \\
\hline $\begin{array}{l}\text { - Adaptability (patient progression, task setting, } \\
\text { and patient group diversity) }\end{array}$ & {$[24,26-30,46]$} & time" $[29]$ \\
\hline - $\quad$ Physical comfort & {$[5,24,25,28,46]$} & \\
\hline
\end{tabular}

\section{Results}

\section{Study Selection}

Initially, 935 references were retrieved from bibliographic databases. After removal of duplicates, 658 potentially relevant papers were screened for retrieval, of which 30 were retained for full text review. After comparing with the selection criteria, 24 of the full text papers were excluded. In total, 3 studies were included via additional reference searches of relevant publications. Therefore, the review includes 9 publications. The number of studies included and excluded at various stages of the review process is shown in Figure 2.2. In all cases, consensus between the 2 raters was reached. Consequently, there was no need to consult the third reviewer. 


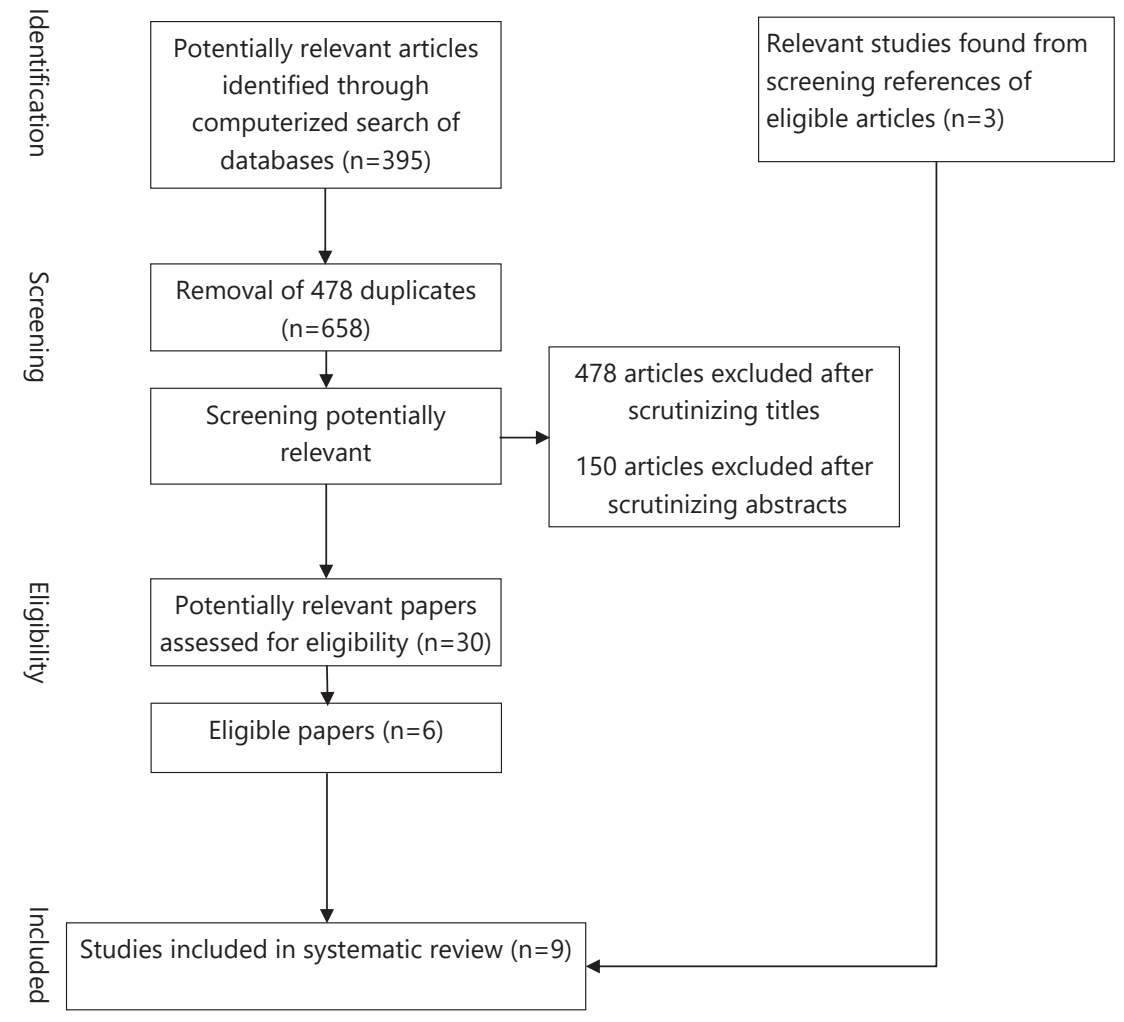

Figure 2.2 Flowchart of study inclusion.

\section{Study Characteristics}

In total, 9 studies covering 139 stroke patients and carers and 384 HCPs were included for analysis $[5,24-30,46]$. The majority of the studies had at most 20 participants except for 2 studies that applied questionnaires involving over 100 participants $[5,46]$. The characteristics of the studies are shown in Table 2.2. All studies described end user's experiences and perspectives regarding the design of AT for use after stroke. In total, 4 studies used interviews [24, 27-29], 3 studies used focus groups [25, 26, 30], and 2 studies questionnaires [5, 46] to elicit information from end users. 
Table 2.2 Characteristics of included studies.

Source and aim of the paper (N=9)
Lam et al.(2015) [30]
Aim: establish the current use and perceptions of
gaming, social media, and robotics technologies
for rehabilitative purposes from the perspective
of adults with upper-limb impairments to identify
barriers and enablers to their adoption and use

Nasr et al.(2015) [28]

Aim: examine stroke patients' experiences of Stroke patients $(n=10) \quad$ In-depth $\quad 8$ and caregivers $(n=8) \quad$ interviews \begin{tabular}{lll}
$\begin{array}{l}\text { Target population } \\
\text { (number) }\end{array}$ & $\begin{array}{l}\text { Method of } \\
\text { data collection }\end{array}$ & $\begin{array}{l}\text { Quality } \\
\text { score }\end{array}$ \\
\hline
\end{tabular} Stroke patients $(n=8) \quad$ Focus groups 9 living with stroke and technology to provide technology developers with insight into values, thoughts, and feelings of the potential users of a to-be-designed robotic technology for homebased rehabilitation of the hand and wrist

Prange et al.(2015) [24]

Aim: identify user requirements for development Stroke patients $(n=5) \quad$ Interviews 5 of an active assistive device to support hand opening during functional activities

Radder et al.(2015) [26]

Aim: identify user requirements as input for the development of a wearable soft-robotic assistive device for the support of hand function of elderly and stroke patients in a wide range of $A D L^{b}$

Hughes et al.(2014) [5]

Aim: understand HCPs', stroke patients', and carers' experience and views of upper-limb rehabilitation and $A \mathrm{As}^{c}$ to identify barriers and opportunities critical to effective translation of ATs into clinical practice

Sivan et al.(2014) [27]

Aim: investigate if the ICF ${ }^{d}$ framework is a useful basis to ensure that the key user needs are identified in the development of a home-based arm rehabilitation system for stroke patients and $\operatorname{HCPS}^{\mathrm{a}}(n=6)$

Stroke patients $(n=4) \quad$ Focus groups

7

and HCPs $(n=7)$

Stroke patients and

Questionnaire 9

carers $(n=79)$ and HCPs

$(n=120)$

Stroke patients $(n=9)$

and HCPs $(n=6)$

Semi-structured 9

interviews 
Table 2.2 Characteristics of included studies. (continued)

\begin{tabular}{l} 
Source and aim of the paper $(\mathbf{N}=\mathbf{9})$ \\
\hline Demain et al.(2013) [25] \\
Aim: investigate stroke patients', caregivers', and \\
stroke professionals' experiences and perceptions \\
of stroke upper-limb rehabilitation and AT use \\
and identify the barriers and facilitators to their \\
use in supporting stroke self-management
\end{tabular}

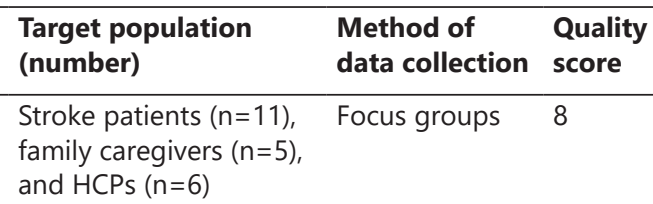

Aim: discover the needs and preferences of therapists with respect to a robot that focuses on upper-limb rehabilitation

aHCP: healthcare professional

${ }^{b} A D L$ : activities of daily living

'AT: assistive technology

dCF: international classification of function, disability and health

\section{Methodological Quality}

Quality scores retrieved from the CASP ranged from 4 to 9 points, with 7 studies having a score above 5 out of a possible score of 10 (Table 2.2). Scores per question of the CASP are shown in Table 2.3. Studies with lower scores tended to provide insufficient information about particularly the recruitment strategy, the relationship between researcher and participants, the ethical procedures, and the data analysis. A minority of the studies (2/9, approximately $22 \%)$ provided information about the role and potential bias of the researcher during the study. Nevertheless, studies with a low quality score were retained for inclusion because of their relevant contribution of data. 
Table 2.3 Questions of the Critical Appraisal Skills Program and the amount of studies that do or do not comply with each question.

\begin{tabular}{|c|c|c|}
\hline Question & Yes & Partially reported or No \\
\hline Was there a clear statement of the aims of the research? & 9 & \\
\hline Is a qualitative methodology appropriate? & 9 & \\
\hline $\begin{array}{l}\text { Was the research design appropriate to address the aims of the } \\
\text { research? }\end{array}$ & 9 & \\
\hline $\begin{array}{l}\text { Was the recruitment strategy appropriate to the aims of the } \\
\text { research? }\end{array}$ & 5 & $4[24,26,29,30]$ \\
\hline Was the data collected in a way that addressed the research issue? & 7 & $2[24,29]$ \\
\hline $\begin{array}{l}\text { Has the relationship between researcher and participants been } \\
\text { adequately considered? }\end{array}$ & 2 & $7[5,24-29]$ \\
\hline Have the ethical issues been taken into consideration? & 6 & $3[24,29,46]$ \\
\hline Was the data analysis sufficiently rigorous? & 8 & $1[29]$ \\
\hline Is there a clear statement of findings? & 4 & $5[24-26,28,29]$ \\
\hline How valuable is the research? & 9 & \\
\hline
\end{tabular}

\section{Synthesis}

Statements and sentences from primary data were discussed and organized into 35 descriptive themes (Figure 2.3). On the basis of the descriptive themes, 5 overarching analytical themes were derived: (1) promoting hand and arm performance; (2) attitude toward technology; (3) decision-making process; (4) usability; and (5) applicability in practice, illustrated in Figure 2.3.

Table 2.1 provides illustrative quotations from included studies and the corresponding descriptive and overarching themes. During third-stage discussions (analytical theme identification) within the multidisciplinary expert group, underlying relationships between those themes were identified (Figure 2.3). For an AT to be considered for the support of the upper-limb function in stroke, the device should address a therapeutic base for promoting hand and arm performance (theme 1). A positive attitude toward technology (theme 2) is a prerequisite for starting the decision-making process (theme 3), whether to use an AT. After it is decided to (consider to) use an AT, aspects determining the usability of the system (theme 4) play a crucial role in the level of user satisfaction. The applicability of an AT in practice (theme 5) depends on factors that may promote long-term use of the device, when properly implemented. 


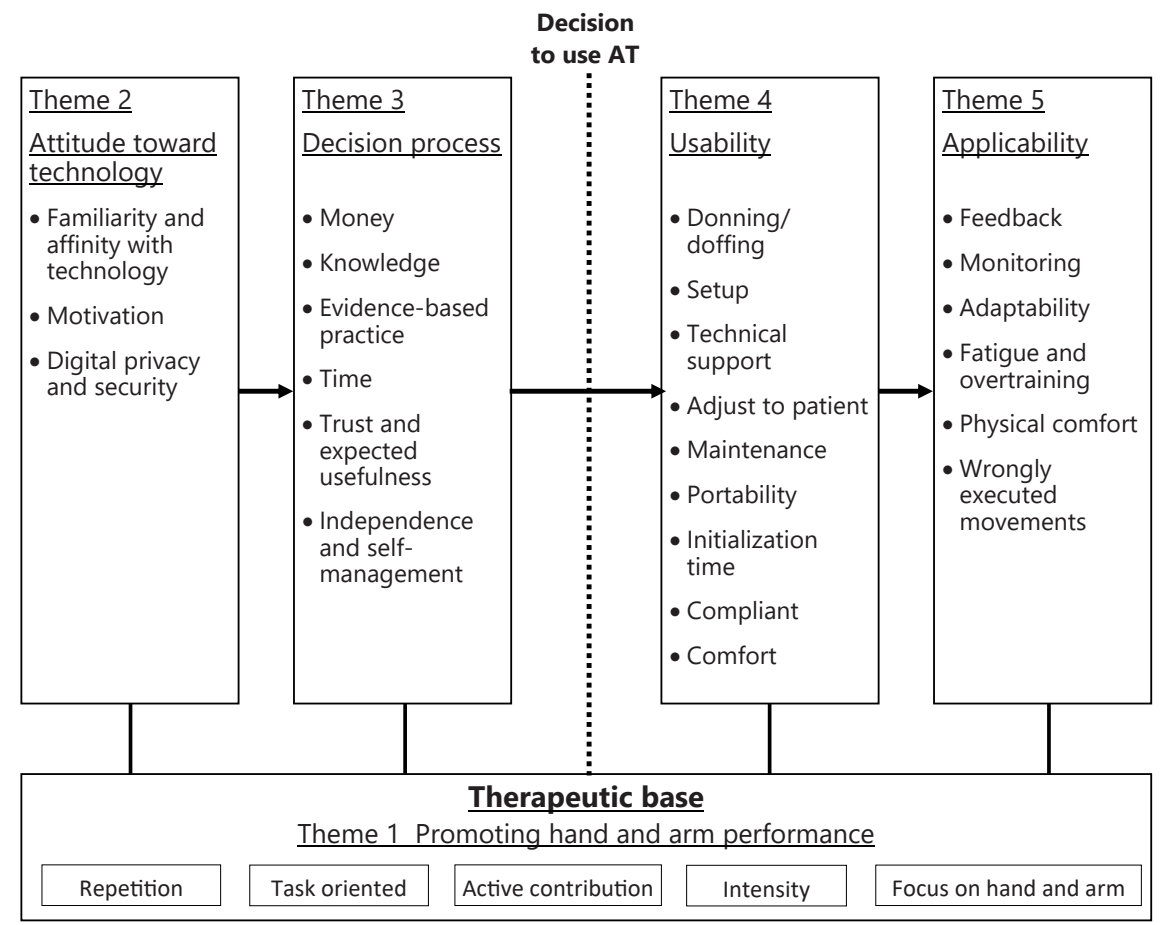

Figure 2.3 Themes in use and implementation of assistive technology (AT) for the arm and hand according to healthcare professionals and stroke patients.

\section{Theme 1 - Promoting Hand and Arm Performance}

\section{Repetition, Task Oriented, Active Contribution, Intensity, and Focus on Hand and Arm}

Therapeutic principles which are the foundation of motoric recovery should be addressed by AT. Stroke patients and carers have remarked that intensive movement repetition needs to be promoted to regain any degree of function and to optimize recovery $[25,28,30]$. In their eyes, meaningful movements are preferred during training $[28,30]$ as they want to improve their ability to use their affected limb in functional activities such as combing hair, washing, dressing, cooking, and eating with knife and fork [27]. HCPs in both qualitative [29] and quantitative studies (99\%) [46] agree that the intensity and frequency of meaningful taskoriented movements should be enhanced. So training should be tailored to the individual goals, which involves training of the specific task to accomplish the goal, and also comprises components of the tasks that stroke patients want to remaster [29]. When severely affected, active contribution and training of the severely affected side is preferred, to achieve the ability to use it as supporting hand in bimanual activities [24, 26], as is wished by stroke patients and carers. Tailored to the stroke patients' functional level, training should range from gross 
to fine manipulation and could be provided by games when these are used for rehabilitation purposes [28].

Technology aimed to be used to support the upper extremity should, therefore, offer variability in exercises and its functionality [29]. Computer exercises should enable (virtual) ADL-specific activities through meaningful and functionally relevant activities (88\%) [46] based on the principles of motor relearning [27]. Normal movement patterns needed for daily activities, active participation of the hand and arm, and frequent movement repetition should be promoted and trained in the games [28]. Games functionality should be as close as possible to the functionality of real analog games [29].

Over $75 \%$ of the stroke patients, carers, and HCPs mentioned that the current practice in therapy is insufficient [5], as there is therapeutic emphasis on the lower extremity [25, 27], whereas additional therapy would enhance their upper extremity functioning [27]. All the end users thought that time efficiency of therapy could be improved with AT allowing additional time for upper extremity training [5].

\section{Theme 2 - Attitude Toward Technology}

Motivation, Familiarity and Affinity With Technology, and Digital Security and Privacy

Before considering using an AT, the attitude toward technology in general can either play a facilitating role or form a barrier, for both the stroke patient or end user and the HCP. Beside the before-mentioned factors to promote hand and arm performance, HCPs stress the importance of training to be motivating and challenging for stroke patients. Motivation of stroke patients to regain control over movements of their affected side is usually very strong [28]. This can be achieved by dividing large goals into smaller, achievable goals, but it can also be enhanced by including a gaming element in the case of therapeutic devices [29]. Games, either web-based or offline as AT, are innovative means that can help to motivate stroke patients to do their therapeutic exercises [28]. Stroke patients, carers, and HCPs acknowledged the motivational aspect of AT as they were seen as an improvement on routine therapy because they are hightech and more enjoyable [25]. All participants, stroke patients and HCPs, in the study of Sivan et al.[27] thought that using a home-based technology aimed at arm exercises would help them to perform more arm exercises. More independence $[27,28]$ and regaining confidence in their own body are motivating aspects for stroke patients to engage in the exercise program [28].

However, mixed feelings are expressed about the affinity with technology [28, 30]. Feelings about AT are considerably influenced by the familiarity with technology; stroke patients with technology experience before they suffered the stroke tend to be more positive toward new 
technologies [28]. Stroke patients are willing to adopt new technologies if they are proven to be effective; however, a longer time is needed for learning to use the technology [30]. Time that some stroke patients do not want to waste [30]. Participants had limited exposure to technology for rehabilitation. Aging has stopped stroke patients from making full use of the benefits of technology [30].

Unlike the younger generation that grew up with the internet, stroke patients are not keen on going online [30]. In fact, security and safety of personal information were primary concerns of stroke patients when talking about connecting social networking websites to homebased rehabilitation technology [30]. Integration of social networking negatively influences the potential acceptance of such rehabilitation programs [30]. Therapists emphasized that a system should be able to save individual settings and data of a stroke patient [29].

\section{Theme 3 - Decision-Making Process}

Knowledge, Evidence-Based Practice, Advice, Time Investment, Safety Aspects and Regulations, Trust and Expected Usefulness, Independence and Self-Management, and Money

The decision-making process for AT consists of factors important to both stroke patients and their carers as well as HCPs. Stroke patients are eager to function independently during ADL through self-management [25]. Stroke patients expect that home-based technology would give them more independence in their rehabilitation program [27]. In addition, stroke patients, carers, and their HCPs mentioned that an AT should be used independently at home [27, 46], without the direct assistance and presence of an HCP (70\%) [5]. Independent use of the AT is something that should be facilitated by the hardware and software design $[25,29]$. The design of the device in terms of safety, such as suitable solutions for emergency situations (back-drivable mechanism and quickly removable from the stroke patient), electrical safety, and safety for the environment, plays a role in the decision-making process as well [24, 27].

Some of the participants are actively engaged in the search for solutions to promote arm recovery $[5,25]$, although there are many stroke patients who have little to no exposure and knowledge about AT [25, 30]. A major part of the HCPs, stroke patients, and carers experience difficulties in accessing training and advice on $\mathrm{AT}$, whereas stroke patients and carers rely on the information given to them by HCPs. Ideally, they would like to seek advice from an HCP they know and trust [25]. However, stroke patients feel that they receive too little information because HCPs lack knowledge and training about the availability of AT, HCPs are overworked, and because the therapists are reluctant to give information about devices that would not be state funded [25]. HCPs feel the tension about informing stroke patients about the existence of a device, which may help, but which is not available from state-funded services [25]. HCPs prefer not to proactively inform stroke patients about AT to prevent stroke patients from 
purchasing an upper-limb AT for which insufficient research evidence is available [25]. For HCPs, scientific evidence is crucial $[5,25,29]$, whereas stroke patients and carers are less interested in the generic scientific evidence [5] and are more willing to accept risks [25]. Stroke patients and carers point out that the evidence should be sought on a case-by-case basis because of the huge variety in the stroke population [25]. There is hope that AT could help stroke patients to regain lost capabilities [28], and despite a potential lack of scientific evidence, HCPs believe that AT can enhance hands on physiotherapy [27].

Although stroke patients are willing to spend time and money on potential solutions [25], the decision-making process to invest in an AT largely depends on the financial commitment they have to make [30]. Concerns were raised by stroke patients, carers, and HCPs about the current lack of financial support for AT and whether they will be cost-effective [5, 25, 27, 30]. The amount of money HCPs, or their institution, would be willing to spend on an AT is less than US $\$ 10,000$ for the majority (81\%) of the respondents [46].

\section{Theme 4 - Usability}

Donning and Doffing, Setup, Initialization Time, Portable, Robustness, Instruction on Exercises, Comfort, Lightweight, Ease of Use, Compliant, Adjustment to Patient, Technical Support, and Maintenance

When a device lacks in usability, using it will be less pleasant, which can ultimately lead to device abandonment. As previously mentioned, independence and self-management are very important to stroke patients. Usability factors that can contribute to independent and pleasant use of the device are (1) easy to setup [5, 25, 27-30], (2) simple to apply [25], (3) easy to don and doff without the aid of others [24-26], (4) quickly initialized [26, 28-30], (5) comfortable to use and wear [5, 24, 26], (6) portable [25, 27, 28, 46], and (7) lightweight [24, 26]. A common generic theme mentioned by stroke patients, carers, and HCPs in almost every paper is the ease of use of an AT [5, 25-30]. This theme comprises simplicity [28, 30], easily programmable [25], intuitive in terms of positioning, easy to operate [26], and short familiarization time [29] of an AT.

To be usable for both stroke patients and HCPs, adjustment to the stroke patient must be straightforward. An AT must comply with both left- and right-side affected stroke patients [28] and concerns are expressed about complex adjustment between stroke patients [25]. Both hardware and software should facilitate adaptation between stroke patients, but it should also be adaptable to the stroke patient's progression over time [24, 29].

For an AT to be used at home, stroke patients and their HCPs want the device to be compact enough to fit in the home environment $[27,28,46]$. The AT must be deployable in a living 
room, kitchen, or bedroom [27] and should not hinder during ADL [24]. Moreover, stroke patients and HCPs should be able to rely on the AT, therefore, it should be durable [5, 29]. As there is a chance of an AT breaking down, it is preferred that access to engineers and to HCPs who have knowledge about the technology is available at any time [27].

\section{Theme 5 - Applicability in Practice}

Monitoring, Feedback, Wrongly Executed Movements, Fatigue and Overtraining, Adaptability, and Physical Comfort

Stroke patients, carers, and HCPs acknowledge that ATs can potentially benefit functioning of stroke patients by providing intensive therapy and a means of self-management [25]; however, factors influencing the implementation define the chances of user acceptance of AT in the long run. All respondents were of the view that ATs are efficient use of therapy time [5] and could be used to promote the usage of the hand and arm at home. Technology with the purpose of promoting hand and arm performance should first and foremost address the therapeutic principles mentioned in theme 1 promoting hand and arm performance. Besides this, stroke patients and HCPs want the possibility of an AT to be used unsupervised at home, which is why monitoring of their progression and provision of feedback are preferred. Among other reasons, monitoring and feedback are needed to halt or prevent wrongly executed movements, which can cause injury or inhibit recovery $[29,46]$. Compensatory movements are most likely to occur when fatigued, so an AT must monitor the state of fatigue of the stroke patient [30]. The ability to monitor stroke patients'performance and quality of undertaken movements is seen as an important requirement to highlight possible problems [26, 27, 29]. Feedback not only plays a role for the HCP but also is key to support self-management [25]. Feedback on performance $[25,26,28]$ and biofeedback were said to be of importance to stroke patients and HCPs. However, stroke patients do not necessarily wish for feedback from the system but rather prefer to receive feedback from the HCP [26].

Individual physical and cognitive impairments that limit the ability of a stroke patient to perform tasks should be considered when applying a system in daily practice. HCPs are worried that different types of support are needed in ADL because of the individual impairments [26]; therefore, an AT must accommodate to the level of impairment and address movements that the stroke patient needs to improve [28]. A modular system might not only fit into the individual needs of impairment level [26] but also technological familiarity [28]. Concerns are also expressed about the potential risk of harm such as secondary tissue changes, obstruction of blood vessels, sharp parts, and high forces that might cause injuries $[5,24,28,46]$.

Besides adjustment between stroke patients, an AT must be adaptable to the stroke patient's progression over time by adapting, for example, the level of difficulty [29], provided resistance 
and assistance $[30,46]$, and the executed movements $[28,46]$. Automatic adaptation of task settings to account for the variation in impairment level is preferred as stroke patients only want assist-as-needed: support only during (parts of) activities that need assistance [24, 26].

\section{Relations Between Factors and Themes}

The previous paragraphs discussed the factors within each of the 5 overarching themes. From the included studies, it is clear that the factors can affect one another and there are also relations between the overarching themes. The main relations between factors and themes are mapped in Figure 2.4.

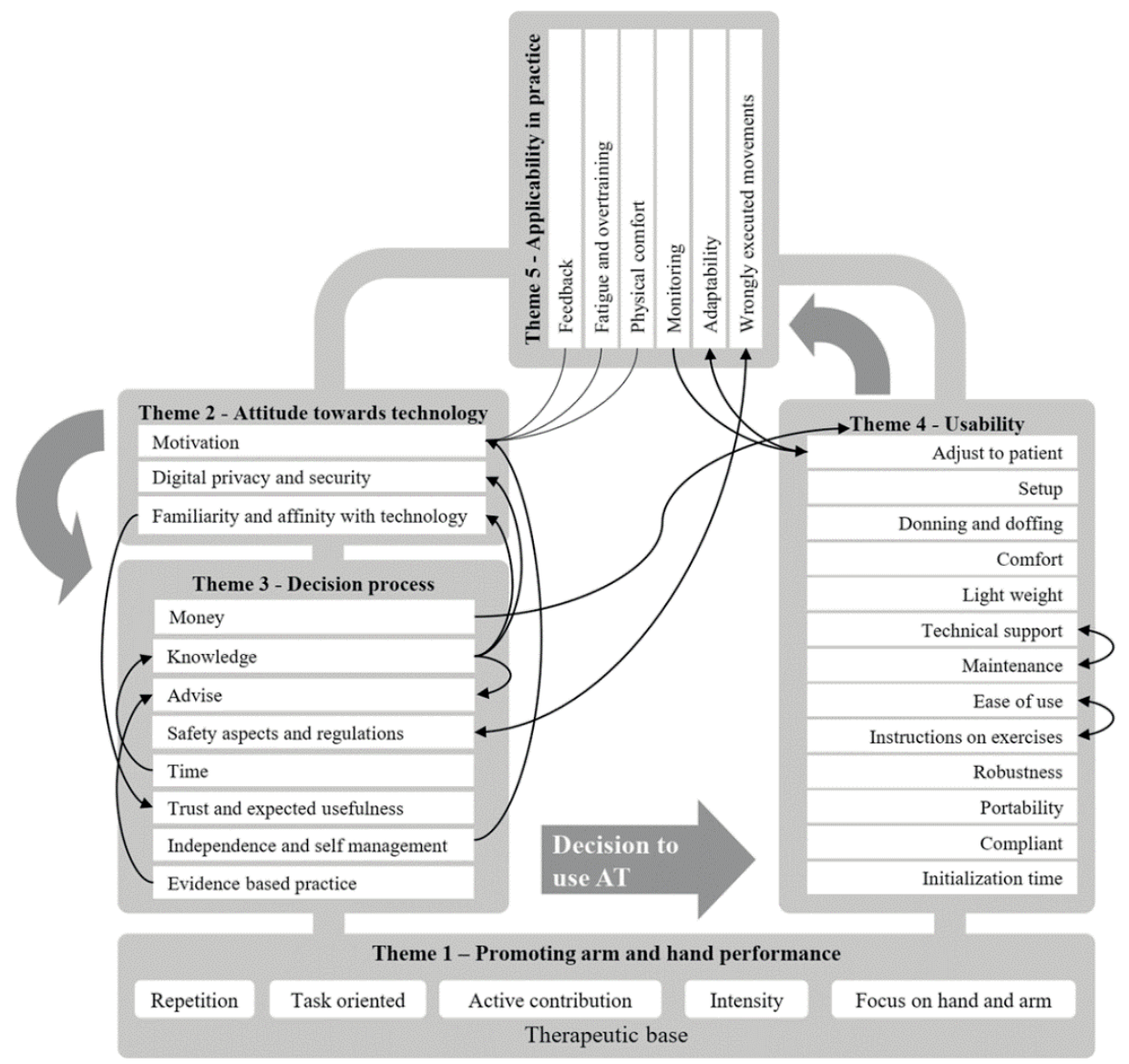

Figure 2.4 Factors and themes influence one another. Connecting lines indicate relationships between factors. AT: assistive technology. 


\section{Use Context of Assistive Technology}

ATs are designed to be used either in the clinic or during daily life in a domestic situation. Although the definition of all themes and factors will differ to some extent between an AT used in the clinic or at home, the most pronounced differences are displayed in Figure 2.5.

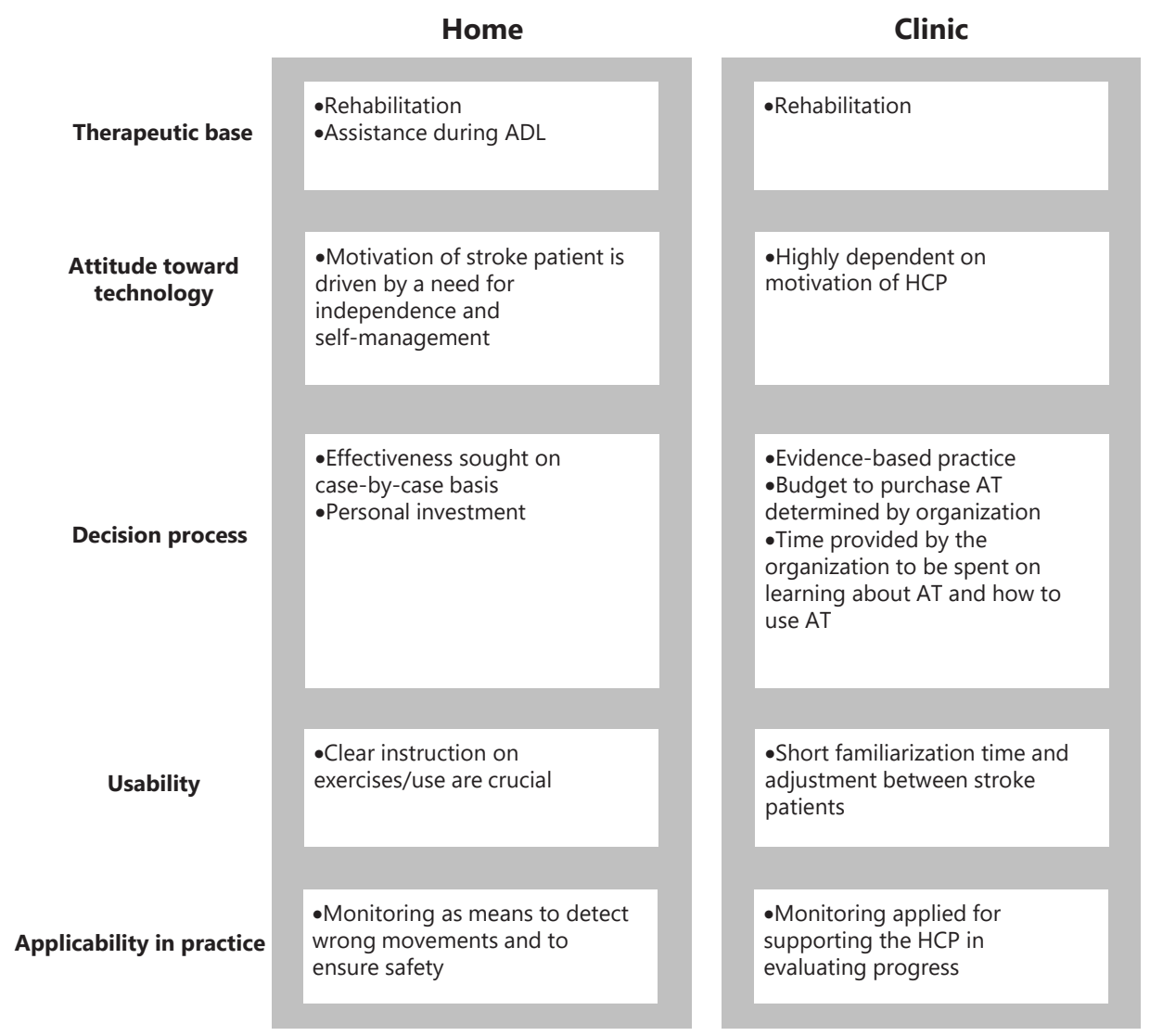

Figure 2.5 The importance and interpretation of the factors may differ depending on the use context of assistive technology. ADL: activities of daily living; AT: assistive technology; HCP:

healthcare professional.

\section{Discussion}

\section{Principal findings}

This review comprehensively investigated user needs, preferences, and expectations that are expected to be associated with acceptance and adoption of AT for promotion of hand and arm performance after stroke. Through a meta-synthesis, 5 overarching themes were identified from literature. Factors relevant to stakeholders who may purchase or decide to use AT are 
covered in the following themes: (1) promotion of hand and arm performance, (2) attitude toward technology, (3) decision-making process, (4) usability, and (5) applicability in practice. Although separately presented by themes, the findings of this review highlighted the diversity and interdependence of the numerous factors influencing the chances of acceptance and adoption of AT, as illustrated in Figure 2.4.

\section{Interdependency of Themes and Factors}

The potential of AT for the upper limb has been recognized by stroke patients, carers, and HCPs [25]. Multiple stakeholders are directly or indirectly involved in the use of AT. Where stroke patients and carers put more focus on self-management, HCPs put more focus on evidence-based practice. However, it is important to address the needs of every end-user category during the design process [34] as involvement of both HCPs and stroke patients will decrease the chance on discrepancy between expected and experienced usefulness. Unsatisfactory user interaction, or moreover, a lack of consideration of user needs, might lead to device abandonment $[5,51]$.

Results from this systematic review suggested that adoption of AT depends on multiple organizational and psychosocial factors and can be influenced at any stage, ranging from attitude toward technology to the practical applicability of AT designed to promote hand and arm performance after stroke. Previously, several general design criteria with a primary focus on usability have been developed [52]. The currently identified themes and underlying descriptive factors reflect many of those established design criteria. Moreover, several additional factors were identified in this review beyond those design criteria addressing predominantly usability, which are mainly represented by the themes attitude toward technology and decision process. Both themes affect the organizational process either by playing a facilitating role or by serving as barrier. Besides that, factors such as age, gender, and voluntariness of use as described by the Unified Theory of Acceptance of Use of Technology influence the chances of adoption of technology [53].

To bring AT design to higher levels of user satisfaction and acceptance, the interdependency of user needs as revealed in this review must be considered in every stage of the design process. This means that addressing one particular aspect of the user perspective will not be sufficient to enhance user acceptance as, that aspect, for example, usability, is influenced by other aspects as well, for example, the budget available to purchase the AT (which is in turn dependent on the use context, for instance). Therefore, when designing AT to promote hemiparetic arm and hand function, the complete specter of themes encompassing the user perspective, as identified in this review, should be addressed. 
Motivation to use AT for upper limb after stroke is driven by the wish for independence and self-management. Therefore, use of AT should have substantial added value for the performance of task-oriented activities with the upper limb. In particular, activities that the stroke patient would normally not be able to perform without assistance should be supported by AT. ATs are seen as efficient use of therapy time [5] and could be used to promote the usage of the hand and arm at home. However, before AT can be applied efficiently, the time required to (learn to) use AT plays a crucial role in the acceptance of AT for stroke patients as well as HCPs. The time it takes for acquaintance is highly dependent on usability aspects such as donning and doffing, initialization time, and time needed to setup the device. Additionally, the practical applicability in terms of time needed to adjust the settings between or within stroke patients affects the chance of acceptance. However, if an AT is effective in supporting self-management, stroke patients are willing to spend time, and if necessary money, on it [25]. Naturally, their willingness is dependent on the financial commitment they have to make. Costs associated with AT, and a potential lack of funding, are seen as one of the major factors influencing the decision on purchasing an AT. In terms of accessibility, concerns not only exist regarding purchasing the equipment and whether the time needed from staff can be billed at the insurance $[29,54]$ but also with regard to informing stroke patients about the existence of a device that may help, but is not available from state-funded services [25].

Cost-effectiveness is seen as a determinant for the adoption of any new treatment [5], it, however, does not automatically guarantee adoption into clinical practice or daily life $[5,55]$. Strength of scientific evidence has also been proposed to be an important factor influencing the translation of rehabilitation research into clinical practice, but there also appears to be a mismatch between the strength of the evidence and the clinical use of AT $[5,56]$.

The decision-making process of HCPs to purchase or use an AT, or even inform stroke patients about AT, is largely influenced by the level of knowledge about AT and the scientific evidence present. The decision-making process of stroke patients is influenced by the HCPs as the primary source of information about AT is their HCP whom they trust. As only $25 \%$ of the devices have been tested in stroke [19], the clinical application and implementation remain low $[54,57]$. Currently, HCPs rely on their own experience with AT because of the absence of clear research evidence [5]. As proposed by Hughes et al.[5], collaboration between clinical and developmental sites, healthcare providers, and the commercial sector would allow for a pragmatic approach for HCPs to learn about AT without awaiting publication, real dissemination, and reception of scientific evidence.

\section{Design Practice}

Currently, the design of robotic technology for stroke rehabilitation tends to be technology- 
driven [32]. The focus on high-tech may jeopardize the consideration for (clinical) needs of the target population, which is a major reason why development can benefit from UCD methods. Unfortunately, manufacturers of medical devices in general can be hesitant in the involvement of users in the later stages of the design process because of perceived barriers in obtaining ethical approval and time constraints, among other reasons [58].

Cherry et al.[59] reported on the perceived facilitators and barrier of stroke patients after use of a hand telerehabilitation system for 3 months at home. Although many reported barriers and facilitators are in line with usability factors identified in this review, stroke patients were able to point out the technical difficulties more specifically after actually using the device in their own homes.

For example, unresponsiveness of the system that required rebooting, limited adhesiveness of the Velcro that was used, and incompatibility with existing furniture. New information about perceived facilitators and barriers as a result of prolonged use of a prototype or product highlights the importance of including user perspectives in the beginning of the design as well as later during evaluation of the prototype or product.

Developers should be aware that not only the prototype of the device itself can be evaluated with users. The instructions for use, commonly created in the wrap-up phase of development when all product details are known, can have great impact on usability. Quality of the user manual can be easily improved by giving several end users some assignments with the manual to determine whether the device can be successfully applied by following the instructions. In case of digital applications, it may be possible to collect user feedback after implementation to continue to improve the device through software updates, but developers need to seriously consider any privacy concerns users have, particularly in case of digital applications.

\section{Study Limitations}

In this review, primary or secondary end users were not included during the sessions in which the overarching themes were defined. Instead, people who have experience in the design of assistive devices participated. Their backgrounds were diverse and with their different roles in device design, it was possible to combine the results into a complete framework that is useful to both developers of AT and those that evaluate or apply AT in practice. Inconsistent terminology about AT used among studies affected our ability to identify relevant studies. An iterative search strategy tailored to the databases was supplemented by scanning the reference lists of potentially relevant papers in an attempt to identify all relevant papers.

In addition, lack of distinction between AT used for therapeutic purposes and AT used during 
ADL in many studies made it difficult to design a framework for both purposes separately. Although the identified overarching themes are applicable in both situations, some factors may weigh heavier than others for either therapeutic or ADL purposes. For example, for a device that is to be used at home by only 1 stroke patient, a low adjustment time is not as crucial as when the device is intended to be used by several stroke patients on 1 day at the clinic. In this review, both focus groups and interviews and user survey studies were included in the meta-synthesis. Although the diversity in methods to elicit user perspectives might have influenced the results or its interpretation, the aim of this review was to include all relevant information on user perspectives about AT for the upper extremity after stroke. Valuable authentic information was retrieved from user survey studies, extending the development of factors and themes with unique data from a large( $r)$ sample of potential users. It may be that the importance of factors varies between studies (or user-interaction methods), but weighing factors could not reliably be assigned in this review. Of the included studies, 2 studies had a methodological quality score below $5[24,29]$. Those studies particularly contained insufficient information about the recruitment strategy, data collection, relationship between researcher and participants, consideration of ethical issues, and provided an unclear statement of findings. Although rated low, those studies contained authentic information that contributed valuably to the comprehensive overview of themes related to user needs for AT for the upper limb as identified in this study. Another limitation is a potential selection bias in the reviewed studies where only participants who were already interested in the use of technology for the upper extremity were included in the study. This may have biased the views expressed by the participants in those studies. On the other hand, the various papers collectively included participants both with and without prior knowledge about and experience with AT.

\section{Future Work}

The 5 themes as identified in this study are relevant to aid future AT developers in quickly determining essential user requirements as a first step of a UCD process. As stated before, the factors identified in this review have interdependency and the importance of a factor may change depending on the use context. Therefore, all factors need to be considered within the specific use context for which an AT is being developed. However, the reviewed studies did not indicate if certain user needs were more important than others. Therefore, insufficient information was present to rank the importance of the factors or themes, but it would be highly relevant to assess the weights that should be attributed to the identified factors and themes in future research. After identification of the user requirements, design solutions can be created and developed [34]. The results gained from the focus groups, interviews, and questionnaires of the studies included in this review primarily reflect the expectations about AT use before actual usage of technology. The chance of actual use of a device is probably related predominantly to the experienced ease of use and perceived usefulness of the system $[24,60]$, 
which cannot always be predicted beforehand. Therefore, subsequent evaluation of the newly designed AT in terms of a priori user preferences and corresponding user acceptance might give new and more specific insights into the (key) user preferences for an AT.

\section{Conclusions}

This systematic review on user perspectives on AT identified several factors and themes that reflect user preferences for AT for the upper limb post stroke, before its development. The findings from this study emerged barriers and enablers influencing the adoption of AT for the upper limb after stroke within the 5 overarching themes; (1) promoting hand and arm performance; (2) attitude toward technology; (3) decision process; (4) usability; and (5) practical applicability. Besides insight into relevant aspects for design of AT, this review showed that those aspects are highly interdependent. A potential purchaser of AT goes through a decision process. Prerequisite for entering the decision process is a sufficient positive attitude toward technology and the desire to increase independence and self-management of the stroke patient. The stroke patient and their carer(s) prefer to consult with a trusted HCP, who may or may not have experience with AT. By combining factors such as money, expected usefulness, and safety aspects, a decision can be reached to purchase AT. If AT incorporates therapeutic principles and can be used pleasantly in a time-efficient and safe manner, chances of acceptance increase. Time efficiency can be increased by usability factors such as setup time, clear and understandable instructions for use, easy donning or doffing, and adjustability. Features such as monitoring fatigue and detecting wrongly executed movements can contribute to safety. Depending on the use context, either at home for ADL purposes or for rehabilitation at a clinic, the importance of each factor may vary.

Due to this interdependency and a lack of weights attributed to the factors in the included studies, a ranking of most important themes could not be established within this review. Therefore, the current framework should be supplemented by future research evaluating the importance of the factors, while also considering differences in use contexts, such as clinical or domestic application of AT. 



\title{
Identification of user requirements for assistive technology for support of upper limb daily life interactions from stroke patients \\ Chapter 3
}

\begin{abstract}
The uptake of assistive technology to improve the functionality of the upper limb in people with disorders affecting the neuromuscular system, such as stroke, is often limited by a gap between the users' needs and the design of the technology. This study aims to identify the technology-specific end-users' requirements for the development of upper limb assistive technology to support daily life activities based on the results of a questionnaire, focus group and specialist consultation. The focus group results showed that unobtrusive support, intuitive use, and adaptiveness to the individual user and his or her disease severity are key for stroke patients. The technology-specific end-user requirements identified in this study can be used to supplement general user requirements identified in the literature, in order to improve the design of assistive technology for support of upper limb function in daily life
\end{abstract}




\section{Introduction}

People with disorders that affect the neuromuscular system, such as stroke, often suffer from difficulties in performing activities of daily living (ADL) due to reduced functionality of the upper limb (UL) $[19,61,62]$. Decreased functionality of the UL leads to a decrease in independence and impacts quality of life $[13,63]$.

The demand for technological solutions, which can support or compensate for loss of functionality in motor function, increases with reduced level of independence and UL function $[12,61,64]$. Nowadays, numerous technological solutions are available ranging from simple assistive tools (e.g., adapted cutlery) to robots that entirely substitute human movements in very severe cases $[22,64,65]$. In theory, personal assistance or (in)formal care can be reduced by $30-42 \%$ through use of assistive technology $[22,65]$. Unfortunately, the preferences and needs of end-users and their environment are often not met in the technical design of the device which results in many users abandoning these devices [5, 12, 25, 66-68]. In order to bridge the translational gap between the users' needs and the design of the technology, a user-centered design needs to be used in the development of the UL assistive technology [26]. Input from end-users at the beginning as well as throughout the design process, as done in a user-centered design, is regarded as effective to enhance the chance for uptake [34].

In the literature, focus is placed on general end-user perspectives for UL assistive technology. If assistive technologies are to be used to support independence during daily life activities, they need to be simple to apply [26, 67-69], easy to use [5, 25, 70, 71], safe [5], pleasurable $[69,70,72]$, of reasonable cost $[46,69,70,73]$, motivating and should be able to provide quantitative and or qualitative feedback [67]. The time taken to prepare, set up and maintain assistive devices are key issues for all stakeholders [5, 25, 69, 70]. For stroke patients and carers the device needs to be easy to get on and off in the presence of a weak, contracted hand/arm as well as intuitive in terms of correctly positioning the device $[25,70]$. The appearance of the device is not seen as important factor for either stroke patients nor healthcare professionals [5]. Thus, for self-management it is critical to incorporate the above mentioned features in the design of a device.

Nowadays, designers are focusing more and more on innovative and technically complex assistive technology. Where development of technology commonly was technology-driven [32], are user-centered design methods increasingly adopted nowadays. The People, Activities, Contexts and Technologies (PACT) framework was invented to cover all aspects of usercentered design including social and technological aspects. Although eliciting end-user input through analysis of the PACT aspects is considered as a useful starting point for design [74], the technological aspect is often neglected. In stroke, gathered end-user-input is often still 
targeted at rather generic information about the envisioned use of the assistive technology, for instance as reflected by the People, Activity and Context domains of the PACT framework $[5,25,72,74]$. There is little emphasis on end-users' views towards specific technical aspects, such as intention detection, options for support, and feedback.

The eNHANCE project aims to assist people with stroke and Duchenne Muscular Dystrophy in performing UL daily life activities with the environment. The focus of this project is on innovative aspects of the technology such as intention detection, performance assessment, and behavioral modelling. Therefore, the aim of this paper is to identify end-user requirements, specifically addressing the technical features, for the development of UL assistive technology. This project used questionnaires and focus groups in two target groups, namely stroke and Duchenne Muscular Dystrophy to determine these end-user requirements.

\section{Methods}

To elicit user input, the PACT framework [74] was used to design questions to be addressed during a disease-specific questionnaire and a focus group with patients, carers and clinicians. To determine the starting point for the technology-specific questions to be discussed during the focus groups, a questionnaire addressing the People, Activity and Context domains of the PACT framework was set up.

\section{Questionnaire}

In order to develop the questionnaire, a literature survey was performed in stroke, to determine the existing body of knowledge regarding user input for UL supporting assistive technologies. The design of the questionnaire was based on published literature on questionnaire design $[75,76]$. Questions and statements relating to the People, Activity and Context were addressed in the questionnaire. Information about patient characteristics (age, sex and time since stroke), their technological affinity and their current hand function was asked to gather information about the People domain. Questions with regard to the current usage of the affected hand and arm complemented with the question which activities they would prefer to perform while involving their affected side provided information relating to the Activity domain. Statements containing information about the Context domain are provided in Table 3.1. 
Table 3.1 Questionnaire statements on the Context domain of the PACT framework, answered on a 5-point Likert scale (from strongly disagree to strongly agree).

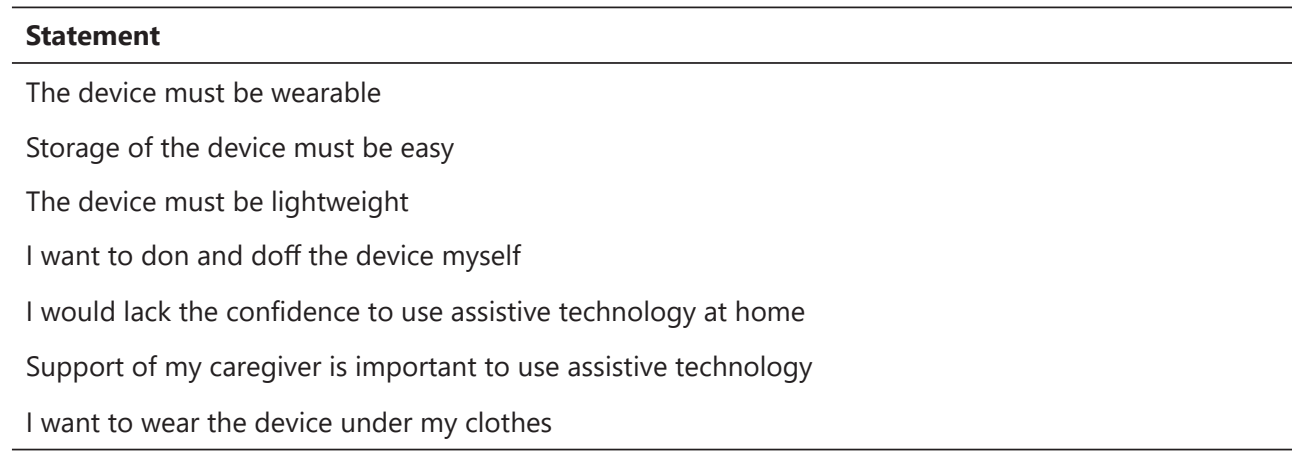

\section{Focus Groups}

A focus group with stroke patients and carers was held in July 2015. Stroke participants were recruited from the Roessingh Rehabilitation Centre, the Netherlands. During the focus group more specific information with regard to the technology was represented by the following themes: support options, intention detection, personalization, feedback and motivational aspects (Table 3.2). Several technical and biomedical experts were present to demonstrate current technologies, and provide feedback about the use of the state of the art technology for the hand and arm.

The focus group took place in the Netherlands. Prior to the start of the study, written informed consent, and agreement for audio-recording of the focus group was obtained from all participants. In the Netherlands, ethical clearance was obtained from the medical ethical committee Twente, in May 2015.

All topics were accompanied by examples (visual where possible) so that the participants could envisage the options more easily. Using an interactive presentation, those items were put up for discussion by asking the participants input via a variety of user interactions, such as voting and ranking, combined with plenary discussions between all participants about their thoughts, ideas, opinions, experiences and expectations.

\section{User requirement identification}

The qualitative data coming from audio-recordings and notes from the focus group was elaborated. Transcripts were discussed between researchers and direct comments were subsequently grouped together. From this, common topics were identified to describe the user perspectives per predefined theme. Thereafter, user requirements were compiled according to preferences expressed by the majority of the participants in each focus group. Subsequently, 
user requirements were discussed between clinical experts, involving rehabilitation physicians, physiotherapists and clinical researchers. All requirements were independently prioritized by at least three clinical experts per target population using the MoSCoW method; Must have (M), Should have (S), Could have (C), Won't have (W) [77]. The MoSCoW method is a technique used for prioritization of requirements with stakeholders to highlight the importance placed on each requirement. The final priority was based on most votes given for the corresponding user requirement.

Table 3.2 Main questions discussed during focus groups.

Introduction project, participants and focus group

Support options

How would you like to be supported by the system?

1. System takes over entire movement

2. System compensates for gravity

3. System supports as needed

Intention detection

Can you imagine in which way a system could detect your movement intention?

Which of the following options would you find best, acceptable and unacceptable:
A. Subconscious
B. Conscious
1. Eye-tracking
1. Voice recognition
2. Sensors (movement/pressure/force)
2. Joystick
3. Muscle activation
3. Pushing a button

Personalization

Would you like to have a system that can adapt itself to your personal preferences?

Can you imagine examples of how such a system could be personalized?

Would you like the system to detect the activity you are performing?

How much time is acceptable for the system to get used to your preferences?

Feedback and motivation

Would you like to receive feedback from the system? If so, about 'how well' and/or 'how much' you performed?

Audio, visual, graphs and tables or vibrotactile?

Would you like to be encouraged by the system to use your upper limb?

\section{Results}

In the result section, the findings from the questionnaire and the end-user requirements as derived from the focus group are presented. In total, ten stroke patients and two partners participated in this study, of which 7 stroke patients filled out the questionnaire. 


\section{Questionnaire}

People Domain Findings from the Questionnaire

Most of the questionnaire respondents were male, above the age of 60 and in the chronic phase after stroke (Table 3.3). Main problems in functional use of the hand and arm were a lack of fine motor skills and control of the hand.

Table 3.3 Primary end-user characteristics.

\begin{tabular}{lllll}
\hline & $\begin{array}{l}\text { Target } \\
\text { population }\end{array}$ & $\begin{array}{l}\% \\
\text { Male }\end{array}$ & $\begin{array}{l}\text { Mean age } \\
\text { (range) }\end{array}$ & Onset since disease in years (range) \\
\hline Questionnaire & Stroke $(n=7)$ & $71 \%$ & $66(45-78)$ & $3.4(2.5-4.5)$ \\
Focus group & Stroke $(n=3)$ & $100 \%$ & $70(67-75)$ & $3.3(2-4.5)$ \\
\hline
\end{tabular}

Activity Domain Findings from the Questionnaire

The majority of the respondents used their affected hand and arm at least sometimes. Dressing and undressing, biking and using the affected hand as supporting hand were the activities in which the most respondents used their affected hand and arm. With regard to activities in which they would like to use their affected arm; domestic chores, eating, drinking and cooking and dressing/grooming were reported most often. Personal hygiene, outdoor activities, mobility but also fine motor skill activities and hobbies were mentioned.

\section{Context Domain Findings from the Questionnaire}

Findings answering the statements of the 'Context' domain of the PACT framework are summarized in Figure 3.1. The majority of the respondents either agreed or strongly agreed that assistive technology should be easy to don and doff, wearable and light weight, and easy to store. All participants would be confident to use assistive technology independent at home. However, opinions about the amount of support needed from the caregiver and whether the device should be worn under the clothes or not were more diversified.

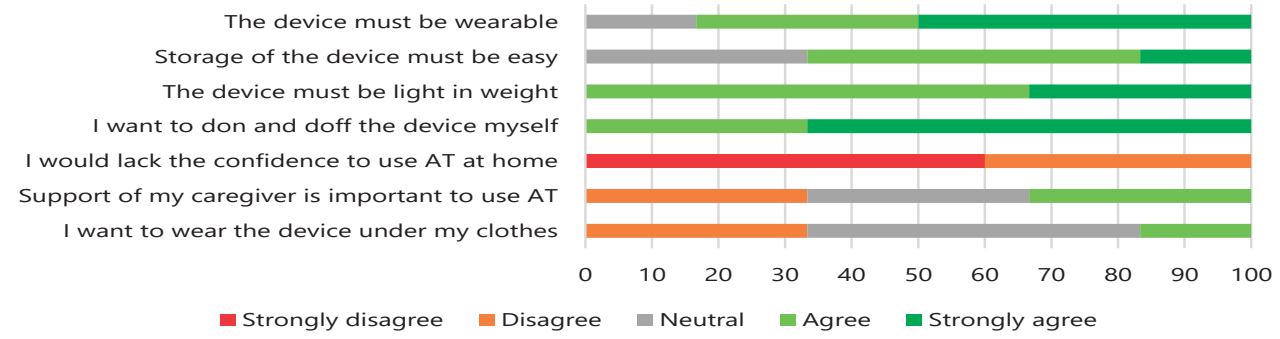

Figure 3.1 Perceptions of stroke patients on contextual questions with regard to assistive technology, expressed in percentages. 


\section{Focus group derived end-user requirements focused on technological aspects}

Three primary end-users (stroke patients) and two secondary end-users (partners) were included in the focus group. Patient characteristics of the stroke patients who participated in the focus group can be found in Table 3.3. In summary, the majority favored assistance only as needed, however, a more severely affected participant preferred the option to self-adjust the amount of support manually since his upper limb was a-functional. Opinions about ways to detect the intention of reach and grasp movements divided with some favoring conscious (e.g., button pressing) over subconscious control (muscle activation, eye-tracking and movement sensors). Participants pointed out that they always want to regain more functionality of the UL, which is not dependent on the severity of the consequences of the stroke they suffered. Therefore, all participants preferred personalization of the system but differed with regard to individual characteristics, which should take into account mainly disease-specific aspects related to nature and severity of motor and/or cognitive limitations, as a basis for personalization. If an assistive technology is to be used during $A D L$, feedback about the quality of their movements is not necessary. According to participants, the non-affected side of stroke patients has taken over many activities, which automatically disables the affected limb even when it is not necessary. Therefore, information about the quantity of use of the affected arm needs to be monitored and, preferably visually, fed back to stimulate participants.

In Table 3.4, a subset of the user requirements resulting from the translation of user expressions from end-users gathered during the focus groups can be found. Only requirements prioritized as must have, or should have are listed in Table 3.4.

\section{Discussion}

Reduced function of the upper limbs in people with stroke impacts their functional independence and quality of life [19]. Although the use of assistive technology is promising, user-centered design methods are needed to include end-users in the design process and to enable development of devices that better suit the needs of the users [26]. Findings from the questionnaire and the user requirements gained from the focus group in stroke provided useful and specific information on the technological features of assistive technologies for the UL from an end-users' perspective.

The functional benefit of a device must be balanced with its burden of use [64]. Minimization of the burden must result from including end-users in the design of the device. In addition to the end-user requirements about technological aspects of assistive technology gained from this study, there are a number of other aspects that are important for the adoption of assistive technology. In line with the findings from the questionnaire performed in this study, assistive 
technologies need to be: easy to use, portable, safe, and easy to don and doff during daily activities such as eating/drinking, preparing food, personal hygiene, as well as supporting hand in stroke in order to gain more independence and perform desirable activities [5, 24-26, 70-73, 78]. As stroke patients prefer to use assistive technology at home, storage of assistive technology must be easy. An assistive technology can preferably be used several times a day depending on the easiness with which the system can be put on by the patient. Accessibility or knowledge about the device is also identified as important factor for the uptake of UL assistive technology [78]. In general, the results of the questionnaire of this study and previous research incorporating input from both primary and secondary end-users are consistent with the 17 design and engineering criteria as set up by Batavia et al.[52].

Table 3.4 User requirements with corresponding priority. $M=$ Must Have, and $S=$ Should Have

\begin{tabular}{lc}
\hline User requirements & Priority \\
\hline Support options &
\end{tabular}

The system must allow the user to use their own existing power, movement and function, rather than replace their function

The amount of the support must consider the existing contractures on the upper M limb

The system must support arm function during reaching and fine motor control of $\quad M$ the hand

The amount of support given by the system must be manually adaptable

\section{Intention detection}

The system must subconsciously detect the intention of the user

The system must have the possibility to switch to conscious control of the user

\section{Personalization}

The system must be adaptable to personal limitations and needs

The system must be able to learn user preferences within one week $\mathrm{S}$

The system must be adaptable to different kind of movements in tasks related to M activities of daily life

The system must adapt support to fatigue

\section{Feedback and monitoring}

The system must not overproduce feedback M M

The system must give feedback about the quantity of the movements $\mathrm{S}$

The system must keep track of performance based on personal capacity and should $\quad M$ use that to motivate the user

The system must motivate the user to be active during the movements M

The system must document progression $\mathrm{S}$ 
Although previous research has identified a need for feedback, (mechanical) adjustment to patients and the ease of use, there has not been a specific focus on the technological aspects of assistive technology $[5,25,73]$. During the focus group, almost every participant pointed out that it is important that their own existing power, movement and function must be enhanced, rather than replaced, by the system in order to be as independent as possible. Within the population, highly individual aspects such as variety in disease severity, in addition to personal preferences and interests, needs to be taken into account during the personalization of interfaces. Therefore, it is of great importance that a system can be personalized not only to the personal preferences and interests of the user, but also to disease-specific needs in the motor and cognitive domains [78].

With regard to detection of the movement intention of the user, our participants predominantly preferred sub-conscious control, with the possibility to switch to or combine it with conscious control. Although subconscious control was most preferred, there was difference between participants in the preferred option (e.g., movement sensors, eye-tracking, muscle activation).

In order to reduce or reverse functional decline in motor function, active engagement during movement and intensive use of the arm or hand are crucial [79]. Although, stroke patients do not feel the urge to receive direct feedback from an assistive technology for home-use during ADL. In order to improve, people with stroke would like to be motivated by the system. In stroke, people can usually use their unaffected arm and hand unobtrusively to perform complex movements [80], which demotivates them to use their affected hand. Awareness of their movements and (possible) non-use of the affected side during daily life activities is important to them.

All patients, family caregivers and healthcare professionals were positive regarding the potential of assistive technologies to facilitate self-management and independence. Although participants did not have experience with assistive technology prior to their attendance, they were capable of describing their preferences, which was possibly due to the presented visuals during the focus group. Yet, it is throughout the entire design process of great importance to monitor whether the actual use matches the end-users' expectancies since they could only express their preferences based on the examples presented during the focus group.

This study provides valuable information about users' views regarding technology aspects of an assistive technology, and relevant insights into the most population-specific topics, despite the small number of participants. In this study, healthcare professionals were included in the prioritization of the user requirements afterwards, but they did not take part in the focus groups. Therefore, our findings as presented in this paper may be different if healthcare 
professionals were included earlier on in the process.

Actual use of a device can be related to the perceived usefulness and ease of use [60]. In order to improve the chance of acceptance, specific attention needs to be paid to ease of use and usefulness as well as a high priority should be given to accessibility and personalization of both hardware and software aspects of assistive technology [24]. The user requirements from this study, focused on technical domains, can be used to complement the existing information on user perspectives identified as important barriers and facilitators for UL assistive technology.

End-user input from this study has highlighted end-user preferences and needs with regard to options to (personalize) support technology designed for the hand and/or arm, to detect the intention of the user, to provide feedback and to monitor progression. This information can be used in the design of newly developed UL supporting assistive technology. Currently, the identified user requirements are being taken into account during the design of an intelligent, adaptive, unobtrusive UL supporting assistive technology within the eNHANCE project, aimed at assisting people with DMD or stroke in independently performing UL daily life activities. 



\title{
Feasibility and user experience of control modalities for a grip-supporting soft- robotic glove: comparing force, muscle activity, voice and wink control with stroke patients
}

Chapter 4

\begin{abstract}
Objective: To assess and to compare user perspectives on, and feasibility of, force, muscle activity, voice and wink to control a grip-supporting soft-robotic glove with stroke patients.

Design: Cross-sectional study

Patients: Ten chronic stroke patients with decreased hand function.

Method: Feasibility of each modality to control the glove was represented by the success rate of controlling the glove, and time needed to complete a reach-and-grasp task. User perspectives were assessed with the System Usability Scale, Quebec User Evaluation of Satisfaction with Assistive Technology, and a semi-structured interview.

Results: The success rate of controlling the glove was highest for muscle activity, followed by voice and wink control. Although highly variable, reach-and-grasp task was performed fastest with force control. User perspectives varied greatly, with four participants favoring force control, while all other modalities were chosen twice as favorite. On average, force and voice control received System Usability Scores above 70, and muscle activity and wink control between 50-70.
\end{abstract}

Conclusions: Due to the large variation in feasibility and user experience between participants, it is not possible to identify one modality as most suitable to control a grip-supporting softrobotic glove. A modular approach for its control is suggested to meet individual preferences and abilities of stroke patients. 


\section{Introduction}

Technological innovations have the potential to enhance the performance of the upper limb of stroke patients directly $[12,81]$. Yet, even existing assistive aids are often abandoned [31]. User-centered design approaches are advocated as a way for technology to better meet the preference of end-users, thereby stimulating adoption of a system [34]. However, frequently a mismatch exists between the expectations of the end-user and the actual design, possibly because the design of newly developed systems is often technology-driven [19].

After a stroke, difficulties with the performance of activities of daily living (ADL), such as eating/drinking, personal care and household activities, are commonly experienced due to a diminished functioning of the hand and arm. One year post stroke, around $40 \%$ of the patients still need assistance in $A D L$, which limits their independence and affects their quality of life $[11,13]$. Assistive technology (AT) that can be used to assist ADL could reduce formal and/or informal care and thereby increase independence $[22,23]$.

So far, a lot of research into technological devices for stroke rehabilitation purposes has been performed, whereas reported developments about AT to be used during daily activities are scarce $[18,82]$. In contrast with AT designed for rehabilitation, AT used as an aid during daily activities should not interfere with the patients' social and physical environment. Therefore, various requirements in terms of usability have to be addressed specifically with this type of technology [83]. Stroke patients and healthcare professionals, highlighted the importance of an AT to be easy to use, portable, easy to set up and intuitive to use, among other factors [5, 24-29].

Up to now, various robotic devices have been designed for use by stroke patients $[12,18]$. Different modalities to control a device, such as manual switches, kinematics, eye-tracking, forces and torques, surface electromyography, auditory sensors and movements of the unaffected limb have shown to be feasible in healthy subjects $[12,84]$. If end-users are involved in the design process to identify user requirements, their involvement usually focuses on a general, conceptual level [83]. Technical details of the mechanical design, actuation options, sensing or control, etc., are only rarely addressed specifically. This leads to the chance that the chosen control modality does not suit the preferences and/or abilities of the user. Therefore, simple and user friendly modalities to control a technology for daily use at home should be tested by end-users to find out what suits the preferences of the end-user best. An interesting question in this context is whether perceived usability and objective performance measures with the device are related. In this study, off-the-shelf, non-invasive, devices were selected to control a commercially available wearable soft-robotic glove, the SEM ${ }^{\mathrm{TM}}$ glove. The goal of this study was to assess and to compare the user perspectives on, and feasibility of, force, 
muscle activity, voice and wink modalities to control a grip-supporting soft-robotic glove. An additional aim was to get insight into the match between subjective experience and objective task performance measures.

\section{Methods}

\section{Participants}

Chronic stroke patients were recruited from a regional rehabilitation center if they had suffered a unilateral stroke more than three months ago and experienced hand function impairments causing problems in the execution of ADL. Additional inclusion criteria were: sufficient cognitive ability to understand two-step instructions, basic knowledge of the English language to understand simple commands to use the voice control modality, more than 10 degrees of active flexion and extension of the fingers, and (corrected to) normal vision. Participants were not eligible for inclusion if they experienced severe sensory problems, were diagnosed with aphasia, had an increased muscle tone (Perceived Resistance to Passive Movement (PRPM) score [85] > 2), experienced severe pain of the affected hand and/or arm, or when comorbidities or contractures limiting functional use of the hand were present. All participants were informed verbally and in writing about the purpose and procedures of the study. Written informed consent was provided by all participants prior to inclusion to the study. The study was approved by the local medical ethics committee Twente, Enschede, the Netherlands (study number NL64511.044.18).

\section{System}

The commercial SEM ${ }^{\mathrm{TM}}$ (Soft Extra Muscles) glove is a wearable soft-robotic glove developed to support grip strength (Bioservo Technologies $A B$, Sweden). The added grip strength is regulated by a tendon-driven mechanism that is controlled by sensor input from tactile sensors (Tekscan, Inc.) at the fingertips of the thumb, index- and middle finger. The glove solely provides force if and when the participant actively initiates contact with an object. The glove was used with its original control mechanism (interaction force between fingers and object) alongside three different system setups. In those setups, muscle activity, voice commands and eye winks were used to control the SEM ${ }^{\mathrm{TM}}$ glove instead of interaction forces.

Muscle activity - In order to control the glove using myoelectric signals, a commercial electromyographic sensor system, the Myo armband (Thalmic Labs, Kitchener, Ontario, Canada), was used. The Myo armband was placed onto the affected forearm with the placement in accordance to the manufacturer's instruction. A short calibration procedure was needed to determine a personalized profile in which the muscle activity pattern of five gestures were obtained. In this experiment, solely one gesture per participant was used to 
activate and deactivate the glove. The decoded gestures were streamed to a custom program in a Robot Operative System (ROS) [86] which was used to operate the glove

Voice - A commercially available voice command device (Logitech USB Headset H390) was used to control the glove by voice. The human voice as recorded by the microphone was detected by a voice detection algorithm which was programmed in a ROS environment. The glove could be activated and de-activated by the command word 'action'. At any time, the command word 'stop' deactivated the actuators of the glove. No user specific calibration was required.

Wink - Information from a binocular eye-tracking glasses SMI ETG 2W (SensoMotoric Instruments, Germany) was used to control the glove by winking. Winks are defined as instances where any of the eyes being closed for at least $400 \mathrm{~ms}$ while the other is kept open [87]. The procedure used to control the glove by winking has previously been described by Noronha et al.[87].

In every setting, the glove was worn on the affected hand and the force applied in the activated condition was set at $3000 \mathrm{~N}$.

\section{Evaluation}

The experiment took place in a controlled environment in the lab of Roessingh Research and Development, Enschede, the Netherlands. Participants visited Roessingh Research and Development once in this cross-sectional study. After explanation of the purpose of the study, motor status of the paretic upper limb was assessed using the upper extremity part of the Fugl-Meyer assessment [88]. Assessment of feasibility and user perspectives of the four control modalities was done during two tasks: a grasp-only task and a simulated ADL task involving reaching to and grasping of an object. The grasp task had to be performed with grip support from the glove controlled by muscle activity, voice and wink. The simulated ADL task had to be performed with force, muscle activity, voice and wink control. The order of the control modalities applied during the measurement was randomized using sealed envelopes. After this, user preferences were assessed.

\section{Grasp task}

Participants were asked to place their hand next to a cylindrical object, positioning the hand such that the object could be grasped without changing the position of the hand. They had to activate the glove based on one of the above mentioned commands to grasp the object. Subsequently, the command to deactivate the glove had to be performed to release the object. Both grasping and releasing the object was performed eight times per modality. The number of successful attempts were noted, as well as the total number of attempts needed to 
activate the glove to grasp, or to deactivate the glove to release the object, were measured to define the success rate. The number of unintended command triggers (false positives) were measured as well. Per person, the average of all movements was used for analysis.

\section{Simulated ADL task}

Participants were asked to grasp a cylindrical object placed upright on a table and move it to a platform. The object was released on a platform and the hand was placed back. The reverse task (grasp the object on the platform and place it onto the table) was performed thereafter. Both tasks were repeated four times with each modality, totaling 8 object replacements per modality. Time needed to perform the simulated ADL task was measured for each modality and each repetition. Per person, the average of all movements was used for analysis.

\section{User preferences}

To complement the objective evaluation, evaluation of each modality in terms of usability and satisfaction was performed with the System Usability Scale (SUS)[89] and the Dutch version of the Quebec User Evaluation of Satisfaction with Assistive Technology (D-Quest)[90], respectively.

The SUS consists of ten items with which the subjective experiences of ease of use was assessed, separately for each modality. The items were scored on a 5-point Likert scale ranging from 'strongly disagree' to 'strongly agree'. The calculated total score of the SUS ranges from $0-100$. Scores below 50 indicate a low probability of acceptance in the field. Promising scores, ranging between 50-70, do not guarantee high acceptance in the field, while a score above 70 does indicate a high probability of acceptance in the field [89, 91].

The Dutch version of the Quest consists of 12 items which can be scored on a 5-point Likert scale. Scores range from 'not satisfied at all' to 'very satisfied'. Questions with regard to service, durability and maintenance were removed from the D-Quest questionnaire as these concepts would not apply in this study. The domains safety, comfort, size, weight and effectiveness were scored, with higher score indicating higher levels of satisfaction.

At the end of the measurement session, a semi-structured interview was conducted comparing the different modalities. The following questions were asked: (1) Which modality could control the movement most precisely?; (2) Which modality worked the fastest?; (3) Which modality was the easiest to use?; (4) Which modality was least strenuous to use? Additionally, participants were asked to rank the modalities from best modality to worst modality. 


\section{Data analysis}

The IBM SPSS Statistics software package version 23.0 was used for statistical analysis. Descriptive statistics (mean \pm standard deviation) were used to describe patient characteristics and the outcome measures. Normal distribution of all parameters were checked with the Shapiro-Wilk test prior to selection of appropriate statistical tests. Subjective SUS scores and objective parameters (success rate and time) were analyzed with a repeated measures ANOVA or its non-parametric equivalent, the Friedman test. In case of significance, additional paired sample t-tests or Wilcoxon signed rank tests were performed for parametric and nonparametric outcome measures, respectively. Correlation between the scores on the SUS and objective measures, success rate and time, was tested with a Pearson's $r$ or its non-parametric equivalent, a Spearman's $\rho$ correlation coefficient. Effects were considered statistically significant for $\alpha<0.05$.

\section{Results}

\section{Participants}

Ten stroke patients participated in the study (Table 4.1). For the five females and five males included in this study, time since stroke varied from 2.5 to 10 years. Six (60\%) of the included stroke patients were mildly affected, and four (40\%) were moderately affected [92]. In six participants, the affected side was their dominant side before they suffered the stroke. One person experienced difficulties with winking, and from one person no successful muscle activity calibration profile could be created. The specific modality for those two persons were omitted from analysis.

Table 4.1 Patient characteristics.

\begin{tabular}{ll}
\hline & Participants $(\mathbf{N}=10)$ \\
\hline Sex (male/female) $^{\mathbf{a}}$ & $5 / 5$ \\
Age (years) $^{\mathbf{b}}$ & $61.0 \pm 7.6(51-74)$ \\
Time post stroke (years) $^{\mathbf{b}}$ & $5.8 \pm 2.3(2.5-10)$ \\
Affected body side (left/right) $^{\mathbf{a}}$ & $5 / 5$ \\
Dominant body side pre-stroke (left/right) $^{\mathbf{a}}$ & $1 / 9$ \\
Fugl-Meyer assessment score $^{\mathbf{b}}$ & $49.8 \pm 7.0(37-57)$ \\
\hline
\end{tabular}

${ }^{a}$ Absolute numbers, ${ }^{\mathrm{b}}$ mean \pm standard deviation (range)

\section{Attempts needed to control the glove}

For controlling the glove, the median success rate was highest for muscle activity, followed by voice and winking respectively (Table 4.2). However, individual differences were large, with 3 people performing better with muscle activity, 5 with voice and 2 with wink. The median 
percentage of false positives ranged from $0 \%$ when controlled by voice to $6.25 \%$ when controlled by muscle activity and winking. No significant difference in success rate was found between the modalities, $\chi^{2}(2)=0.45, p=0.80$.

Table 4.2 Number of attempts needed to control the glove, false negatives (FN), and false positives (FP) presented for each modality per individual. Hyphens indicate that the modality was not tested.

\begin{tabular}{c|c|c|c|c|c|c|c}
\hline & \multicolumn{2}{|c|}{ Muscle activity } & \multicolumn{2}{c|}{ Voice } & \multicolumn{2}{c}{ Wink } \\
& Success rate & FP (\%) & Success rate & FP (\%) & Success rate & FP (\%) \\
\hline P01 & $\mathbf{0 . 5 7}$ & 6.25 & $\mathbf{0 . 6 7}$ & 0 & $\mathbf{0 . 5 5}$ & 12.5 \\
\hline P02 & $\mathbf{0 . 8 4}$ & 0 & $\mathbf{0 . 7 3}$ & 0 & $\mathbf{0 . 8 4}$ & 62.5 \\
\hline P03 & $\mathbf{0 . 6 4}$ & 6.25 & $\mathbf{0 . 4 4}$ & 0 & $\mathbf{0 . 9 4}$ & 0 \\
\hline P04 & $\mathbf{0 . 8 4}$ & 0 & $\mathbf{1 . 0 0}$ & 6.25 & - & - \\
P05 & - & - & $\mathbf{0 . 7 6}$ & 0 & $\mathbf{0 . 9 4}$ & 6.25 \\
\hline P06 & $\mathbf{0 . 8 9}$ & 0 & $\mathbf{0 . 7 3}$ & 0 & $\mathbf{0 . 7 6}$ & 37.5 \\
P07 & $\mathbf{0 . 8 4}$ & 6.25 & $\mathbf{0 . 8 9}$ & 0 & $\mathbf{0 . 5 3}$ & 0 \\
\hline P08 & $\mathbf{0 . 9 4}$ & 12.5 & $\mathbf{0 . 8 4}$ & 0 & $\mathbf{0 . 4 8}$ & 18.8 \\
\hline P09 & $\mathbf{0 . 7 0}$ & 37.5 & $\mathbf{0 . 8 8}$ & 0 & $\mathbf{0 . 8 0}$ & 0 \\
\hline P10 & $\mathbf{0 . 8 4}$ & 12.5 & $\mathbf{0 . 9 4}$ & 0 & $\mathbf{0 . 8 9}$ & 0 \\
\hline Median & $\mathbf{0 . 8 4}$ & 6.25 & $\mathbf{0 . 8 0}$ & 0 & $\mathbf{0 . 8 0}$ & 6.25 \\
\hline
\end{tabular}

\section{Simulated reach-and-grasp task}

Time needed to perform the simulated reach-and-grasp task varied among participants as well as among modalities (Figure 4.1). The average time needed to perform the task was the lowest when performing the task controlled by the original pressure sensors of the SEM Glove. Time needed to perform the task with the glove supported by the pressure sensors was shorter than the time needed with all other modalities $(p \leq 0.01)$. 


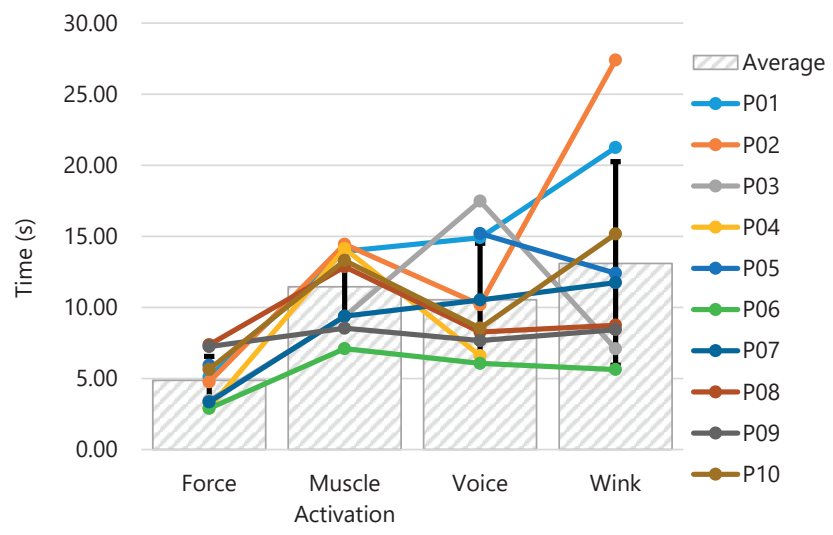

Figure 4.1 Individual and mean time needed to complete the simulated reach-and-grasp task, presented per modality. The error bars indicate the standard deviation.

\section{User preferences}

Preferences of participants varied greatly (Figure 4.2). Four participants out of 10 ranked force control highest, while each of all other modalities had highest rank in two participants. Force control was never indicated the lowest ranking option, while wink- and muscle activity controlled grasping were ranked lowest by four and three out of 9 participants, respectively. In general, the most preferred option was selected based on the perception of (de)activating the glove exactly when desired (sense of control over the glove).

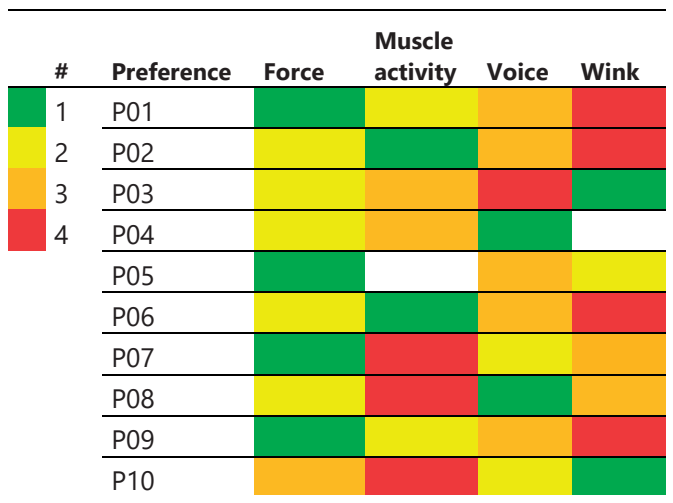

Figure 4.2 Ranking of the modalities per participant. 1 indicates the best option, 4 indicates the least preferred option. Blank fields indicate that the modality is not tested. 
Individual and mean SUS are displayed per modality in Figure 4.3. Mean SUS above 70 were found for force- $(77.8 \pm 13.04)$ and voice- $(71.8 \pm 12.6)$ controlled grasping, while muscle activity- (55.0 \pm 21.7$)$ and wink- (62.2 \pm 16.5$)$ controlled grasping scored between 50 and 70 points on SUS. A significant difference in SUS score was found between the modalities $(F(3$, $21.0)=5.0, p<0.01)$. The SUS score for force-controlled grasping was significantly higher than for control by muscle activity $(\mathrm{t}(8)=4.19, \mathrm{p}=0.03)$ and wink $(\mathrm{t}(8)=3.21, \mathrm{p}=0.01)$. In addition, muscle activity scored significantly lower on the SUS than wink $(t(8)=-2.39, p=0.04)$.

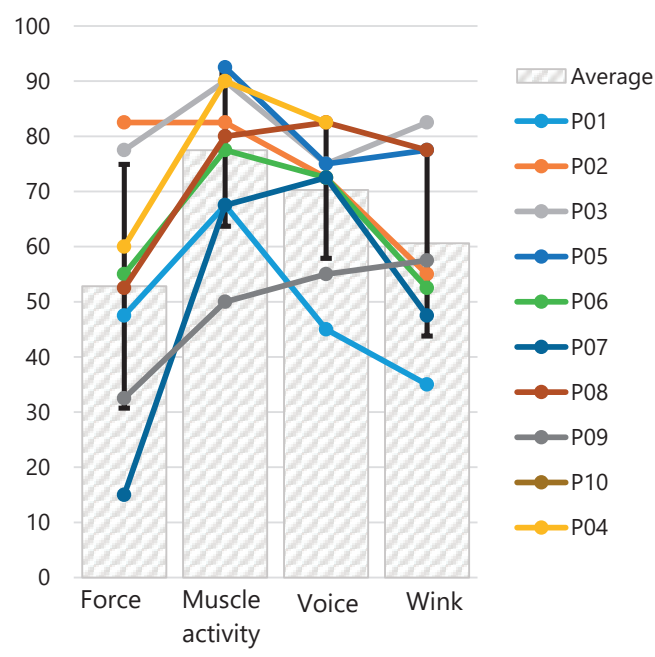

Figure 4.3 Individual and mean System Usability Scores per modality. The error bars indicate the standard deviation.

Overall, participants indicated that grasping controlled by pressure sensors could control the movement most precisely, worked the fastest, was most easy to use, and was least strenuous to use (Table 4.3).

Table 4.3 Preferences rated from 1 (best option) to 4 (least preferable). Averages (SD) are presented for each modality per question. The best option per question is displayed in bold font.

\begin{tabular}{lcccc}
\hline & Force & $\begin{array}{c}\text { Muscle } \\
\text { activity }\end{array}$ & Voice & Wink \\
\hline Which modality could control the & $1.6(1.0)$ & $2.7(1.0)$ & $2.6(1.0)$ & $2.9(1.2)$ \\
movement most precisely? & $1.3(0.5)$ & $2.9(0.8)$ & $2.8(1.2)$ & $2.8(1.0)$ \\
Which modality worked the fastest? & $1.9(1.0)$ & $2.6(1.1)$ & $2.4(1.1)$ & $2.9(1.2)$ \\
Which modality was the easiest to use? & $1.7(1.0)$ & $3.0(0.8)$ & $2.3(1.0)$ & $2.8(1.3)$ \\
Which modality was least strenuous to use? & & &
\end{tabular}




\section{User satisfaction}

The majority of the participants was satisfied with the weight, size, comfort, ease of use and safety of all modalities as expressed in D-QUEST scores (Figure 4.4), except for some aspects of wink and voice control. Specifically, at least half of the participants was less than (quite) satisfied with the size of the eye-tracker for wink control, and the safety of the eyetracker or the voice modality. Most satisfied were participants with the weight of muscle activity and force control modalities, and with the ease of use of the voice control modality.

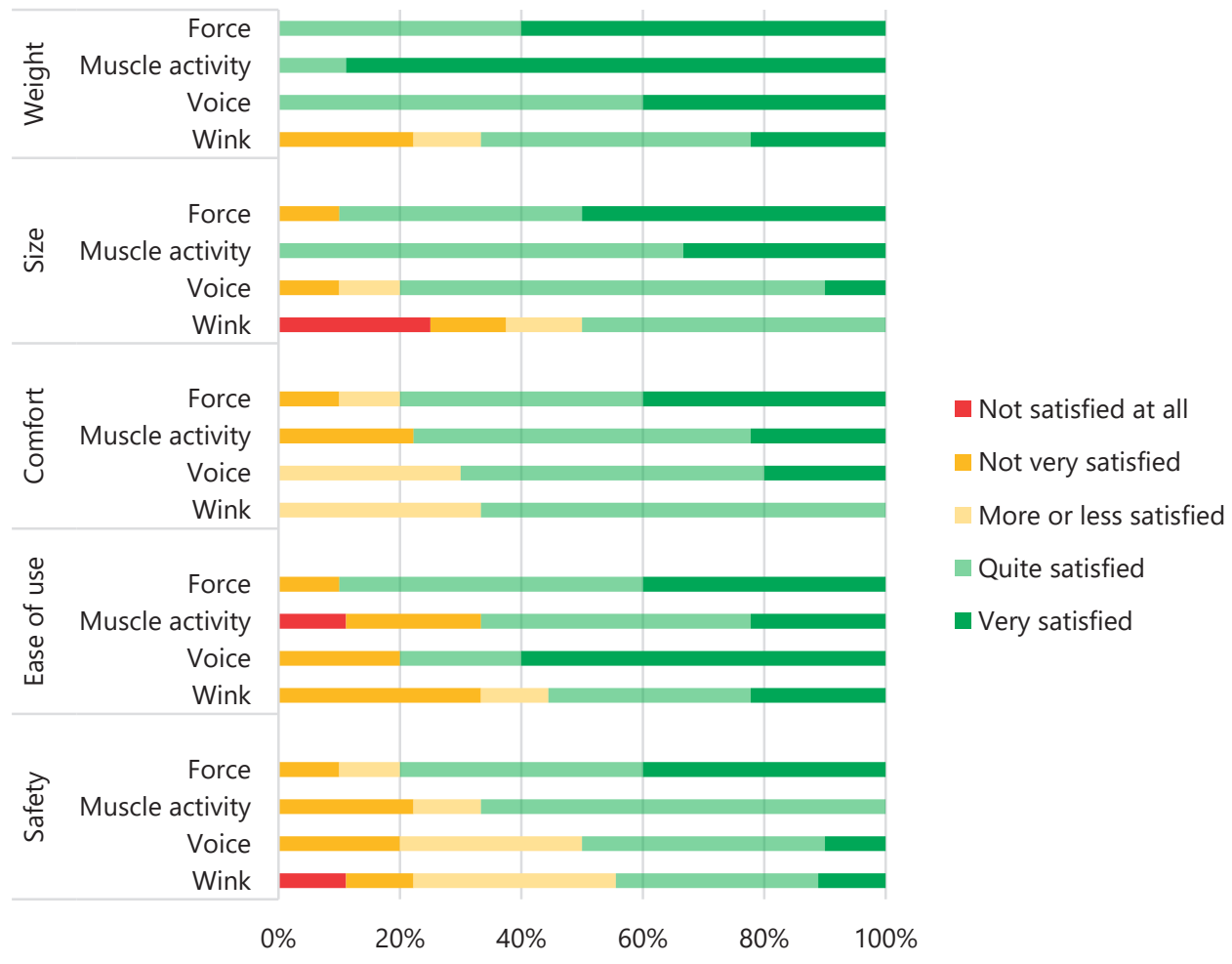

Figure 4.4 User satisfaction per modality scored on five domains.

\section{Correlation between subjective usability score and objective measures}

When assessing the correlation between the SUS scores and the two objective measures, number of attempts and time, weak, but significant, negative correlations are found $\left(r_{s}=-0.37\right.$, $\mathrm{n}=28, \mathrm{p}=0.05$ and $\mathrm{r}=-0.35, \mathrm{n}=38, \mathrm{p}=0.03$, respectively). 


\section{Discussion}

In the current study, we assessed and compared user perspectives on, and feasibility of, force, muscle activity, voice and wink controlled grasping by a soft-robotic glove. The results show that preferences and performance of the end-users are highly variable. Success rate of controlling the glove was similar for each modality. The percentage of false positive ranged from $0 \%$ when controlled by voice, to $6.25 \%$ when controlled by muscle activity and winking. The simulated ADL task was performed faster when the glove was controlled by the pressure sensors. In addition, a discrepancy appeared/seems to exist between user experience and user performance after first-time use of the wearable soft-robotic glove controlled by either muscle activity, pressure sensors, voice or wink.

Nowadays, focus is placed on the development of robot-assisted rehabilitation of the hand, yielding promising results [19]. However, the vast majority of the developed rehabilitation devices for the hand are tested solely in a healthy population during development or are not tested at all [19]. It is plausible that results from healthy subjects are not transferable to the stroke population. In the study of Noronha et al.[87], muscle activity was outperformed by wink and voice when assessing the error rate of grasping in healthy subjects. In the present study, we did not observe a statistical significant difference in success rate of the three modalities, but individual false positive rates were higher in wink controlled grasping when compared to the other modalities.

The discrepancy between performance of healthy subjects and stroke survivors can be explained by the fact that several physical, emotional and cognitive symptoms can be experienced due to a stroke. Besides the differences in functional performance between healthy subjects and stroke survivors, there also is a large variation in the experienced symptoms within the stroke population [7]. In addition to the experienced hand-arm motor execution difficulties, some of the participants in the current study had difficulties with voice while others were, for example, not able to wink properly. Since the location of stroke with resulting hand-arm motor execution difficulties is generally in the area of the middle cerebral artery, it is not uncommon to have problems with speech due to central facial palsy.

The large variation in the stroke population was also reflected in user experience outcomes. In general, participants were satisfied with the weight, comfort, and ease of use of all modalities. Although grasp controlled by the pressure sensors received the highest SUS score, all options were chosen at least twice as the best option. These findings clearly point out the problem to develop a single design that would fit everyone. This has already been pointed out by endusers describing their perceptions about existing wearable robotic devices for the upper limb in the study of Elnady et al.[39], and similar results were found when men with Duchenne 
Muscular Dystrohpy used different control modalities to control arm movements [93]. Endusers in their study suggest that a one-size-fits-all device does not exist since every stroke survivor has his/her own situation [39]. Stroke survivors in another study also pointed out that evidence should be sought on a case-by-case basis due to the variation in the stroke population [25]. This was also reflected by the results of the current study.

There seems to be a discrepancy between subjective and objective outcome measures after first-time use of the wearable soft-robotic glove controlled by either muscle activity, pressure sensors, voice or wink. Participants' attitude towards a control modality was mainly influenced by the intuitiveness of use and the perceived feeling of confidence in the glove ensuring that the object was tightly grasped, instead of based on the objectively measured performance. The mismatch between subjective and objective outcome measures has already been found in research assessing upper limb function of stroke survivors [94]. A recent systematic review highlighted that user experience is not solely depending on objective efficiency and feasibility of a device, but also depends on numerous other factors including familiarity with technology, usability aspects, and confidence in the device [83]. Although it is difficult to assess feasibility of a device after short-term usage because of a limited familiarization, first impressions are created. Even though no significant differences were found in the success rate between control modalities, a clear distinction in user preferences was seen. The current findings therefore indicate the need of considering, and testing, a modular system, at least in terms of control modalities.

Results of this study should be interpreted with caution due to the explorative character of the study, and the inclusion of a small group of stroke survivors with variable severity levels as measured with the Fugl-Meyer assessment. Furthermore, off-the-shelf devices were used in the current study. All modalities have the potential and possibilities to be personalized to the capabilities of the user. Personalization, and a combination, of the control modalities could be tested in future research to reach higher levels of usability, effectiveness, and user experience. Also, long(er) term usage of the wearable soft-robotic glove controlled by the modalities should be investigated in the future to avoid the possible effect of lacking familiarity with the system.

\section{Conclusion}

The current study provides a first insight into the user experience on, and feasibility of, force, muscle activity, voice and wink controlled grasping by a soft-robotic glove in stroke patients. Due to the large variation in user performance and user preferences, it seems not suitable to develop a one-size-fits-all robotic device. The current results indicate the 
importance of involvement of end-users in testing of prototypes of components, already early in the development process, to elicit highly useful, practical and valuable user input for further development. After first-time use of the soft-robotic glove controlled by either of the modalities, there appears to be a discrepancy between subjective and objective outcome measures. Future developers should consider development of modular systems, specifically concerning the input modality for control of a medical device, to enable actual tailoring of the system to the abilities and the preferences of the end-user. 



\title{
Quantifying upper extremity performance with and without assistance of a soft- robotic glove in elderly patients: a kinematic analysis
}

Chapter 5

\begin{abstract}
Objective: To explore the direct influence of a soft-robotic glove on movement duration and movement execution of elderly with decreased hand function during a reach-and-grasp task.

Design: Cross-sectional study.

Patients: Eight subjects, aged 55+, with decreased hand function.

Method: The direct effect of the glove was explored with kinematic analysis during a reachand-grasp task with a light (100 grams) and heavy (1000/2500 grams) cylindrical object, performed with and without glove.

Results: No difference in total movement time between performance with and without glove was found. With glove, relative time needed to transport the heavy object was shorter, while relative time needed to grasp the heavy object was longer. Additionally, transporting light objects occurred with a lower peak velocity and larger elbow extension, and grasping of the object involved a larger hand opening as compared to without glove.

Conclusion: As expected, no positive influence of the glove on total movement duration of elderly was found. The influence of the glove on movement execution varied with movement phase. The found positive and negative effects are possibly due to a perceived confidence while carrying heavy objects with glove or compensation for loss of sensation, respectively. This information can be used for design improvements.
\end{abstract}




\section{Introduction}

The function of the aging hand decreases as a result of loss of muscle mass (i.e., sarcopenia) [95], rheumatoid arthritis or age-related diseases [96, 97]. Symptoms of a decrease in hand function in the elderly population often include loss of grip/pinch strength, joint stiffness, decreased range of motion and increased fatigue or pain [98-101]. This loss of function results in limitations in performing activities of daily living (ADL), such as carrying heavy objects [98, $102,103]$.

An effective approach to improve hand function in daily life is exercise training. Exercise training for older adults with reduced hand strength should consist of components that contain progressive resistance and functional exercise [104]. Another approach to improve functional independence is the use of assistive devices [105], especially when exercise treatment does not solve all physical problems and people are left with, or experience, a diminished hand function. These assistive devices are available in different shapes and sizes, ranging from simple aids (e.g., a jar opener) that supports a specific task, to technological innovations that allow more functionality in daily life. Most of these robotic assistive devices consist of complex, bulky and expensive equipment, while often substituting upper limb movement of the user by robotic action [12].

Wearable assistive devices are focused less on substituting the user and more on assisting use of the hand where needed during ADL. An example is the soft-robotic ironHand glove, developed to support grip strength during ADL [106]. The soft-robotic ironHand glove has been evaluated regarding feasibility (in terms of user acceptance and usability) [107, 108] and its effect on functional performance [106, 108, 109], showing promising results on user acceptance and usability. Although pinch strength increased significantly, the daily life activities, such as picking up, and moving, objects, were performed slower with the ironHand glove compared to without the ironHand glove $[108,109]$. Clinical scales that score performance time may be less suitable to assess the direct effect of a soft-robotic glove on hand function, since other aspects than performance time can be influenced by use of such a glove that is developed to support grip strength. For instance, small changes in movement execution can make a difference in functional use of the hand in daily life $[110,111]$.

Assessing functional tasks through kinematic analysis is useful for evaluating actual functioning of the upper limb in daily life, since kinematics movement analysis is seen as a sensitive and objective method to assess differences in movement execution [112]. Therefore, the goal of the present study was to explore differences in movement duration of a reach-and-grasp task with and without the assistance of the ironHand glove. Secondly, the influence of the glove on duration of movement phases, movement smoothness, trunk displacement, peak hand 
velocity, hand opening and joint excursion of the elbow and wrist were explored.

\section{Methods}

\section{Participants}

A subgroup of 8 participants that also participated in an earlier cross-sectional study, investigating the overall orthotic effect of the ironHand glove [106], were included in this explorative study at Roessingh Research and Development (RRD), Enschede, the Netherlands. Inclusion criteria for participation in this study were: at least 55 years of age, experienced difficulties with performing $A D L$ involving the hand, the most-affected hand is the dominant hand, able to perform at least 10 degrees of active flexion/extension movement of the fingers, sufficient cognitive function to understand two-step instructions, (corrected to) normal vision and living at home. Exclusion criteria were: severe sensory problems, pain or wounds on the hand that may create problems when wearing the glove, severe contractures limiting passive range of motion, co-morbidities limiting functional use of the arms/hands, insufficient knowledge of the Dutch language to understand the purpose or methods of the study. All participants gave their written informed consent prior to the start of the study. The study was approved by the Medical Ethical Committee in Twente, the Netherlands (CCMO-number NL56746.044.16).

\section{ironHand system}

The ironHand glove is developed to support grip strength of the thumb, middle finger and ring finger (Figure 5.1) [106]. The ironHand system consisted of a three-fingered wearable soft-robotic glove (Figure 5.1, A) and a control unit (Figure 5.1, B) that contains the embedded software to control the amount of force needed to support grip strength and the batteries. The control unit was attached at the belt of the participant (Figure 5.1, B). Sensory input from pressure sensors (Interlink Electronics, Camarillo, California, US) at the fingertips is used to control the amount of extra grip that is regulated by a tendon-driven mechanism. An intention detection logic ensures that the grip is activated in a natural and intuitive way with more grip support supplied when a stronger grip is applied on the object. The gain of the control mechanism (i.e. sensitivity) and maximal amount of support from the glove can be tuned for each individual. In this study, the maximal amount of grip strength support was set at $20 \mathrm{~N}$ for each participant and the gain of the control mechanism was tuned for each patient between two preprogramed modes based on the participants' needs and experienced comfort. 


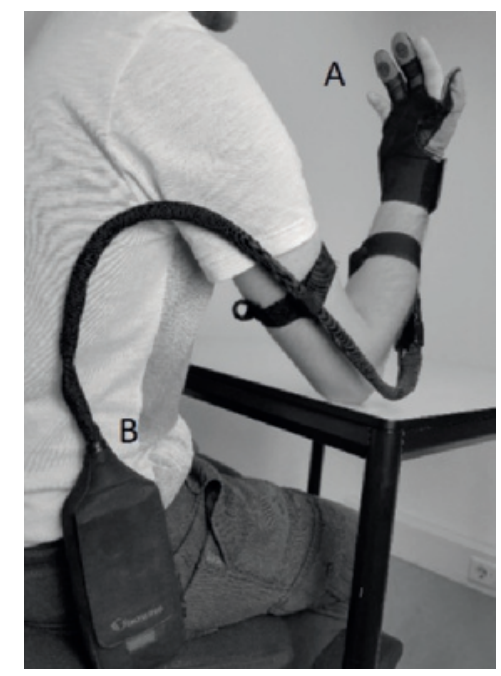

Figure 5.1 The ironHand system. A: Control unit, B: Glove.

\section{Study design}

Prior to performance of a standardized reach-and-grasp task, maximal handgrip strength was measured (without glove) to describe the degree of functional limitations of the present sample $[113,114]$. Additionally, information about the participants, such as gender, age, affected body side, dominant side and handgrip strength, was gathered. Next, participants performed a standardized reach-and-grasp task with various weighted cylindrical objects during a cross-sectional evaluation session. Both tasks were performed with the most-affected hand, once with and once without the ironHand glove, to evaluate differences in movement between both conditions using a 3D motion analysis system. Sealed envelopes were used to randomize the order of glove use (first with or without the glove). The primary focus was placed on total movement duration. Secondary, the effect of the glove on movement duration of movement phases, movement smoothness, trunk displacement, peak hand velocity, hand opening and joint excursion of the elbow and wrist was explored.

\section{Maximal handgrip strength}

Maximal handgrip strength of the most-affected hand was measured with a Jamar hydraulic hand dynamometer (Patterson Medical Ltd., Warrenville, IL, USA) with the handle position set at four for all subjects. The positioning of each subject was standardized as described by the American Society of Hand Therapists [115]. The participant had to squeeze the handgrip of the dynamometer maximally for 5 seconds. Handgrip strength was expressed in kilograms $(\mathrm{kg})$. The subject had three attempts, which were separated by at least 60 seconds rest. The average of the three attempts was used for analysis. 


\section{Standardized reach-and-grasp task}

Before participants started with the reach-and-grasp task, they were instructed how to use the ironHand system properly and they tried it for a few minutes until they felt comfortable with its use. Next, participants performed the standardized reach-and-grasp task (see Figure 5.2 for the experimental set up) to assess movement execution with and without the ironHand glove. In the starting position, each participant was seated with the upper arm aligned with the trunk, the elbow flexed 90 degrees and the palm of the hand positioned on the middle of the table at a predefined start position. The cylindrical object was placed in front of the hand and a platform was positioned within the maximal reaching range of motion of the participant. The task involved: (1) grasping a cylindrical object and, (2) moving the cylindrical object to the predefined position on the platform, (3) releasing the object at the platform and (4) returning the hand to the predefined start position. The task was performed with two differently weighted cylindrical objects (diameter $=5 \mathrm{~cm}$ ) of respectively 100 grams (light condition) and either 1000 grams or 2500 grams (heavy condition), both with and without glove. The light condition was included to assess the effect on movement execution without the weight of the object interfering with the execution of movement. The heavy condition was included to simulate an ADL task, in which usually weight is involved. In each condition, the task was repeated three times. Prior to the start of the measurements, participants had to lift an object of 2500 grams once to the platform/off the table. If they succeeded, the task was performed with the 2500 grams weight, otherwise, the task was performed with 1000 grams instead. The order of cylindrical weight was randomized.

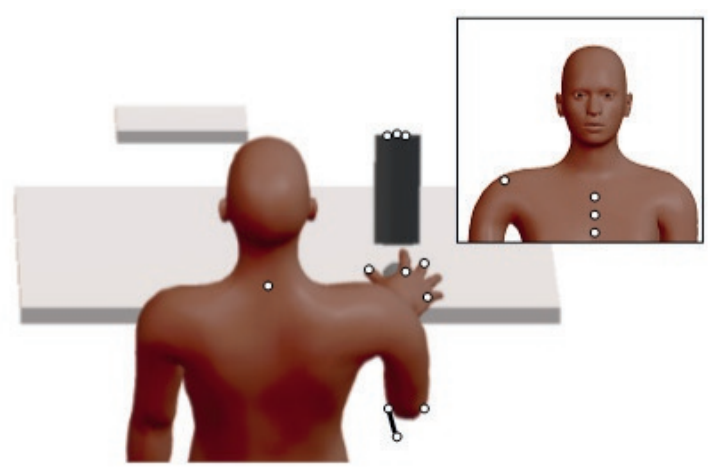

Figure 5.2 Schematic measurement setup with marker placement.

\section{D motion analysis}

During all trials, movements of the trunk and upper extremity segments were captured with six infrared cameras of the motion capture system VICON MX13+ (Oxford Metrics, Oxford, 
UK) by recording the position of reflective markers. In total, fifteen reflective markers were placed on the hand, arm, thorax and neck (Figure 5.2), according to the guidelines of the International Society of Biomechanics [116]. Additionally, three markers were placed on each of the cylindrical objects to record their movements during the task.

\section{Data analysis}

The recorded movement data were analyzed using VICON nexus 1.8.2 and transferred to MATLAB software (R2015a, MathWorks Inc., Natick, Massachusetts, USA) for further custom analysis. All position data were low pass filtered with a second order Butterworth filter of 20 $\mathrm{Hz}$ with zero phase shift.

\section{Segmentation}

The recorded data started at the first, and ended at the last moment that the tangential velocity of the hand exceeded $2 \%$ of the maximum velocity of the hand based on the second metacarpal marker [117]. From that recording, four task phases were identified as described below and presented in Figure 5.3.

1. The start of the grasping phase was identified through either the position of the cylindrical object relative to the hand or the velocity profile of the object within the timeframe between index 2 and index 3 (see Figure 5.3). The indices were chosen at $15 \%$ of the maximal velocity of the hand to facilitate the determination of the movement phases. The position of the object relative to the hand reaches a minimum when an attempt is made to grasp. Additionally, when the object's velocity is larger (mean + two times SD) for the first time than when the cylindrical object stood still, it is likely that the cylindrical object is moved by an external source. The start was set at the lowest frame number of the two options. The end of grasping was defined as the last frame number of either;

I. the difference in velocity of the object relative to the hand marker was smaller than the mean value minus two times the standard deviation of the velocity of the object as measured when that object was not moved. During the reach-with-object phase, the hand and cylinder were expected to have the same movement pattern. The difference in velocity of the hand and cylinder will therefore be minimal;

II. the latest minimum of the vertical position of the object in time between index 2 and index 3 (Figure 5.3). Before the object was lifted in vertical direction, a minimum was seen in the vertical position profile of the object;

III. the combined XYZ-position of the object is larger than two times the standard deviation plus the mean combined $X Y Z$ value of the object when 
not moved and if fifteen frames later the XYZ position of the object is more than twelve times the standard deviation plus mean of the object when not moved. If grasped, the object's movement was not necessarily in the vertical direction. In the case that the object was moved because of touching instead of measuring the end of grasping, the condition that the object had to be moved substantially (twelve times the SD + mean) some frames later was built in.

2. The start of the reach-with-object phase coincided with the end of the grasping phase. The end of this phase was set at the frame number when the object touched the platform. This event was chosen, because the object was always lifted higher than the height of the platform, after which the impact of the object with the platform caused a minimum in the vertical position of the object.

3. Releasing started at the end of the reach-with-object phase and ended with the last frame number of either;

I. the first time that the difference in position of the object and hand marker exceeded the minimal distance, as determined in the grasping phase, between object and hand plus 0.15 times the standard deviation, or

II. the last time that the velocity of the object exceeded the mean velocity plus two times standard deviation of the object as measured in rest.

4. The start of the final reach coincided with the end of the releasing phase and the end of this phase occurred at the first time that the tangential velocity of the hand was smaller than $2 \%$ of the maximum speed of the hand [117].

The grasping and releasing phases were manually checked by comparing the frame numbers to the visualized VICON data. If the algorithm and manual check deviated more than $0.05 \mathrm{~s}$ parameters associated with that grasp or release phase were removed from further analysis.

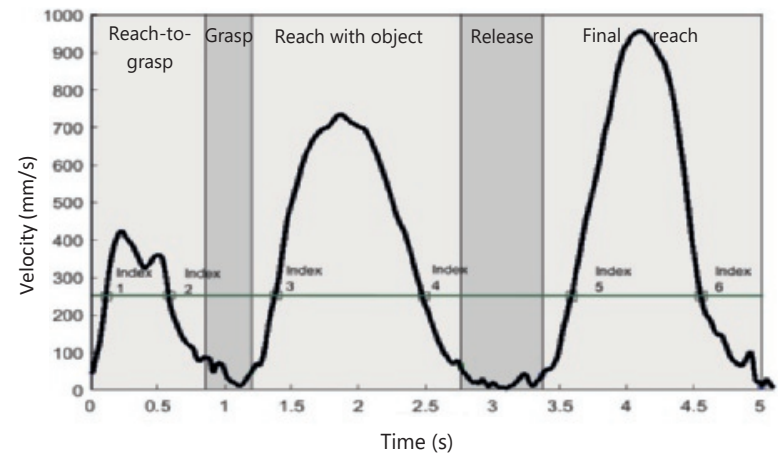

Figure 5.3 Division of tangential velocity profile of the hand marker in five phases. The horizontal line represent the threshold used for the detection of the five phases of the reach-and-grasp task (schematic representation). 


\section{Outcome measures}

The primary outcome measure, total movement duration, was calculated from the initiation of grasping until the end of the final reach. Secondly, the influence of the glove on time needed to execute each of the four phases was calculated in absolute and relative (percentage of total movement time) duration. The time prior to grasping was used to calculate the mean and standard deviation of the motionless cylindrical object. Outcome measures comparable to previous studies performing kinematic analyses of a reach-and-grasp task were calculated to assess the influence of the ironHand glove on movement execution [118-120]. Smoothness of the movement, expressed in the number of movement units (NMUs) [121], was calculated over the entire movement. Local minima and maxima in the tangential velocity profile of the marker on the second metacarpal head were searched for the determination of a movement unit. The difference between a consecutive minimum and maximum with an amplitude of 20 $\mathrm{mm} / \mathrm{s}$ or more indicated a velocity peak that corresponds to the smoothness and efficiency of movement [121]. A movement unit was identified when the time between two consecutive peaks exceeded 150 milliseconds [118]. Maximal trunk displacement (TD) was defined as the maximal 3D displacement of the trunk marker during the task when compared to the initial position in rest. Maximum speed during the reach-with-object phase was calculated from the tangential velocity profile of the hand based on the marker positioned at the head of the second metacarpal. Maximal hand opening prior to grasping was calculated as the maximal distance between the thumb and middle finger marker. The elbow angle during the entire task was calculated from the angle between the vector of the upper- and lower arm. Joint excursion of the elbow was determined by subtraction of the smallest angle from the largest angle between those vectors. Maximum elbow extension angle was measured, and determined as the largest angle between the upper- and lower arm. Excursion of the wrist was calculated by subtracting the smallest angle from the largest angle between the forearm and hand in flexion and extension direction.

\section{Statistical analysis}

Individual values across participants were averaged per task, glove condition and weight of the cylindrical object. Values per parameter were reported as median with interquartile range (IQR, 25th- 75th percentile). Due to the small sample size, differences between performance with and without the ironHand glove were non-parametrically tested for all kinematic variables using the Wilcoxon signed-ranks test. Effects were considered significant for $\alpha \leq 0.05$. No correction for multiple testing was applied since the nature of the study was explorative [122]. Effect sizes were calculated $(r=Z /(\sqrt{ } N))$ indicating a small $(r \leq 0.3)$, medium $(r \leq 0.5)$ or large $(r \geq 0.5)$ effect. IBM SPSS Statistics 23 was used for the statistical analysis. 


\section{Results}

\section{Participants}

Eight adults between 59 and 79 years old participated (Table 5.1). All participants experienced difficulties in performing daily activities due to heterogeneous diseases that affect hand function. Six participants were diagnosed with rheumatoid arthritis/osteoarthritis, one participant had Multiple Sclerosis and one participant had carpal tunnel syndrome. Baseline handgrip strength data indicate 'weak' handgrip strength for all participants, based on cut-off points related to increased risk for mobility limitations [123]. Four participants were able to lift 2500 grams.

Table 5.1 Demographic characteristics.

\begin{tabular}{|c|c|}
\hline & $N=8$ \\
\hline Gender (F/M) & $8 / 0$ \\
\hline Age (years) ${ }^{a}$ & $65.5(62.3-76.5)$ \\
\hline Most-affected body side (right/left/both) ${ }^{b}$ & $5 / 1 / 2$ \\
\hline Dominant side(right/left) & $7 / 1$ \\
\hline Handgrip strength $(\mathbf{k g})^{\mathrm{a}, \mathrm{c}}$ & $11.5(8.0-18.0)$ \\
\hline
\end{tabular}

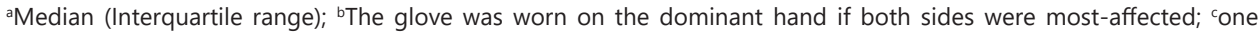
missing value

\section{Movement execution}

Movement execution parameters averaged over all subjects are presented in Table 5.2. A typical example of task execution, in terms of movement time, movement phases, speed and movement smoothness, is presented in Figure 5.4.

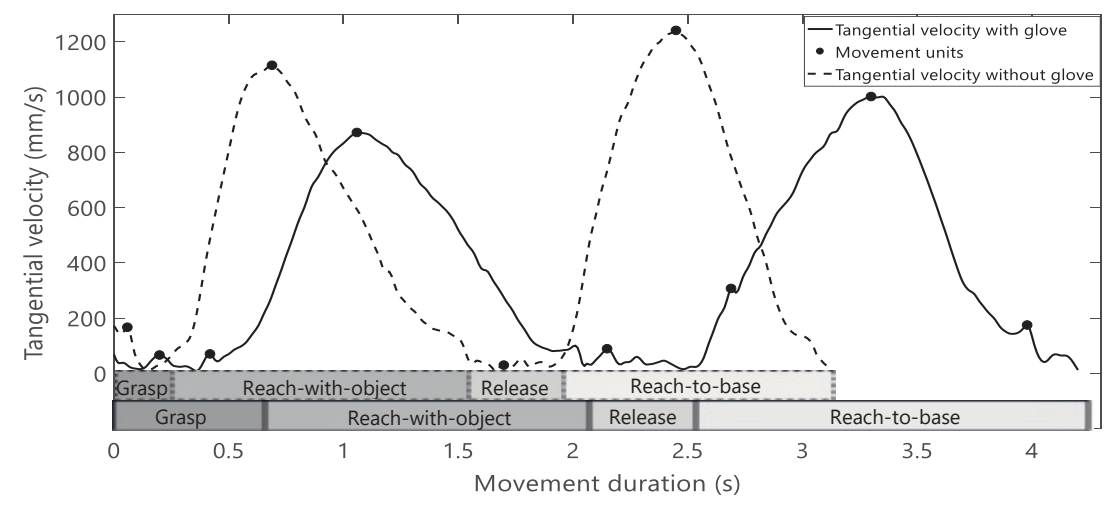

Figure 5.4 Representation of a typical example of total movement duration, absolute duration of movement phases and movement execution with and without glove. 
No negative influence on total movement duration was found (Table 5.2). After division of the movement into phases, an increase of the relative time needed to grasp the object and a decrease in time needed during the reach-with-object phase with glove compared to without glove ( $p=0.05$ and $p=0.04$, respectively) was found only in the heavy condition (Figure 5.5).

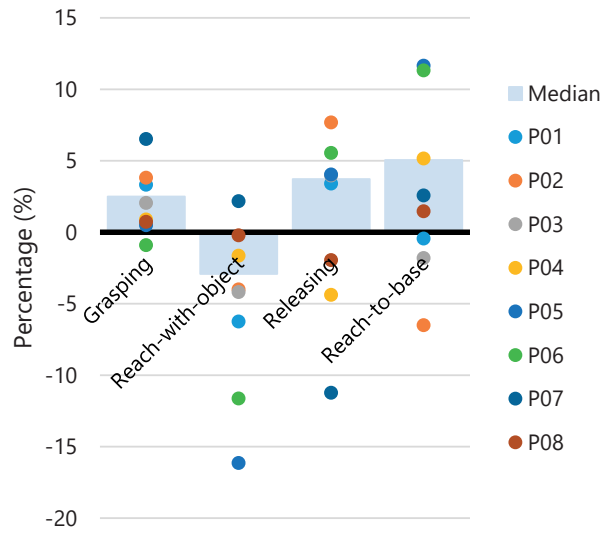

Figure 5.5 Difference scores (with minus without glove) of the heavy object are presented per participant and group median for the movement time for the grasping, reach-with-object, releasing and reach-to-base phases relative to the total movement time.

Peak velocity was smaller with support from the glove in the light condition $(p=0.01)$, but this effect was not observed with the heavy object (Figure 5.6).

Maximum hand opening was larger in the reach-to-grasp phase of the light object while receiving support from the glove $(p=0.05)$ (Figure 5.6).
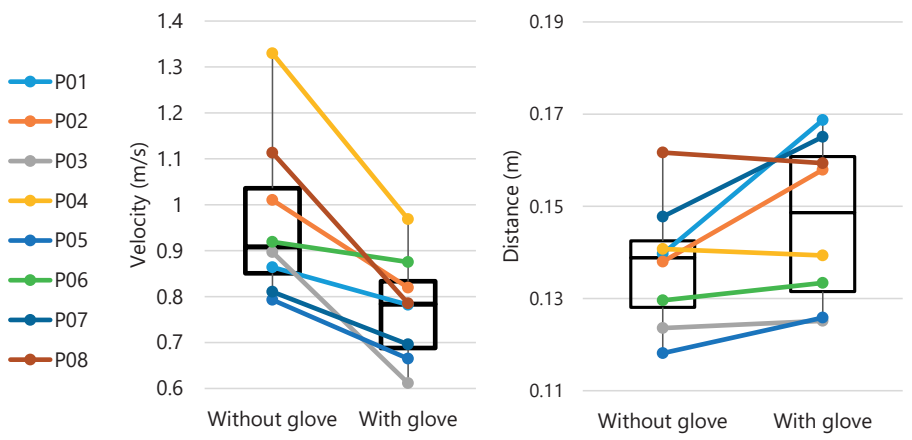

Figure 5.6 Individual scores per participant (lines) and group boxplot with and without glove of peak velocity (left) and maximum hand opening distance (right), using the light object. Note that the $y$-axes do not start at 0 . 
No differences in either movement smoothness or trunk displacement were found when comparing with and without glove in both the light and heavy condition (Table 5.2).

No significant differences in joint excursion of the elbow and wrist were found with glove when compared to without glove, except for a larger elbow extension excursion and a larger maximum elbow extension angle in the light condition $(p=0.04$ and $p=0.01$, respectively).

Table 5.2 Median (IQR) values of kinematic variables without and with glove.

\begin{tabular}{|c|c|c|c|c|c|c|}
\hline & $\mathbf{N}$ & $\begin{array}{l}\text { Weight } \\
\text { (g) }\end{array}$ & $\begin{array}{c}p \\
\text { value }\end{array}$ & $\begin{array}{l}\text { Effect } \\
\text { size }\end{array}$ & Without glove & With glove \\
\hline \multirow{2}{*}{ Total movement time (s) } & 8 & Light & 0.33 & -0.25 & $3.64(3.04-4.37)$ & $4.22(3.94-4.54)$ \\
\hline & 8 & Heavy & 0.09 & -0.42 & $4.43(4.17-5.42)$ & $4.80(3.96-5.70)$ \\
\hline \multirow{2}{*}{ Phase 'Grasping' (s) } & 7 & Light & 0.50 & -0.18 & $0.25(0.15-0.35)$ & $0.24(0.21-0.36)$ \\
\hline & 8 & Heavy & 0.12 & -0.38 & $0.49(0.39-0.86)$ & $0.65(0.38-1.00)$ \\
\hline \multirow{2}{*}{ Phase 'Reach-with-object' (s) } & 8 & Light & 0.12 & -0.38 & $1.25(1.04-1.49)$ & $1.49(1.14-1.59)$ \\
\hline & 8 & Heavy & 0.48 & 0.18 & $1.64(1.42-2.00)$ & $1.49(1.28-1.72)$ \\
\hline \multirow{2}{*}{ Phase 'Releasing object' (s) } & 8 & Light & 0.07 & -0.46 & $0.38(0.26-0.55)$ & $0.50(0.44-0.68)$ \\
\hline & 8 & Heavy & 0.33 & -0.25 & $0.87(0.45-1.21)$ & $0.93(0.65-1.31)$ \\
\hline \multirow{2}{*}{ Phase 'Final reach' (s) } & 8 & Light & 0.07 & -0.46 & $1.49(1.35-1.63)$ & $1.70(1.63-1.82)$ \\
\hline & 8 & Heavy & 0.07 & -0.46 & $1.29(1.24-1.77)$ & $1.64(1.51-1.90)$ \\
\hline \multicolumn{7}{|l|}{ Total movement time (\%) } \\
\hline \multirow[b]{2}{*}{ Phase 'Grasping' (\%) } & 7 & Light & 0.74 & -0.09 & $6.88(4.85-9.56)$ & $6.35(5.64-8.15)$ \\
\hline & 8 & Heavy & 0.05 & -0.49 & $\begin{array}{l}10.54(8.61- \\
16.38)\end{array}$ & $\begin{array}{l}13.04(10.79- \\
17.67)\end{array}$ \\
\hline \multirow{2}{*}{$\begin{array}{l}\text { Phase 'Reach-with-object' } \\
\text { (\%) }\end{array}$} & 8 & Light & 0.58 & -0.14 & $\begin{array}{l}38.03(29.17- \\
41.57)\end{array}$ & $\begin{array}{l}38.02(32.79- \\
39.49)\end{array}$ \\
\hline & 8 & Heavy & 0.04 & 0.53 & $\begin{array}{l}35.15(32.66- \\
45.68)\end{array}$ & $\begin{array}{l}32.23(29.02- \\
35.91)\end{array}$ \\
\hline \multirow{2}{*}{ Phase 'Releasing object' (\%) } & 8 & Light & 0.26 & -0.28 & $\begin{array}{l}12.13(7.30- \\
15.25)\end{array}$ & $\begin{array}{l}13.60(11.81- \\
15.02)\end{array}$ \\
\hline & 8 & Heavy & 0.58 & -0.14 & $\begin{array}{l}15.11(12.95- \\
27.34)\end{array}$ & $\begin{array}{l}18.83(16.31- \\
21.79)\end{array}$ \\
\hline \multirow{2}{*}{ Phase 'Final reach' (\%) } & 8 & Light & 0.58 & -0.14 & $\begin{array}{l}39.64(37.69- \\
48.35)\end{array}$ & $\begin{array}{l}41.95(37.70- \\
45.62)\end{array}$ \\
\hline & 8 & Heavy & 0.26 & -0.28 & $\begin{array}{l}31.22(29.35- \\
36.92)\end{array}$ & $\begin{array}{l}36.27(32.60- \\
40.18)\end{array}$ \\
\hline \multirow{2}{*}{$\begin{array}{l}\text { Movement smoothness } \\
\text { (NMU) }\end{array}$} & 7 & Light & 0.61 & -0.14 & $4.33(4.00-6.50)$ & $5.00(6.00-7.33)$ \\
\hline & 5 & Heavy & 0.79 & -0.09 & $7.33(5.67-13.25)$ & $9.33(4.75-14.17)$ \\
\hline
\end{tabular}


Table 5.2 Median (IQR) values of kinematic variables without and with glove. (continued)

\begin{tabular}{|c|c|c|c|c|c|c|}
\hline & $\mathbf{N}$ & $\begin{array}{l}\text { Weight } \\
\text { (g) }\end{array}$ & $\begin{array}{c}P \\
\text { value }\end{array}$ & $\begin{array}{l}\text { Effect } \\
\text { size }\end{array}$ & Without glove & With glove \\
\hline \multirow{2}{*}{ Peak velocity (m/s) } & 8 & Light & 0.01 & 0.63 & $0.91(0.82-1.09)$ & $0.78(0.67-0.86)$ \\
\hline & 8 & Heavy & 0.16 & 0.35 & $0.77(0.60-0.87)$ & $0.74(0.62-0.80)$ \\
\hline \multirow{2}{*}{ Max. hand opening (m) } & 8 & Light & 0.05 & -0.49 & $0.14(0.13-0.15)$ & $0.15(0.13-0.16)$ \\
\hline & 8 & Heavy & 0.07 & -0.42 & $0.14(0.12-0.15)$ & $0.15(0.13-0.16)$ \\
\hline \multirow{2}{*}{$\begin{array}{l}\text { Max. trunk displacement } \\
\text { (m) }\end{array}$} & 8 & Light & 0.40 & -0.21 & $0.20(0.10-0.26)$ & $0.18(0.10-0.25)$ \\
\hline & 7 & Heavy & 0.24 & 0.32 & $0.25(0.12-0.33)$ & $0.25(0.10-0.28)$ \\
\hline \multicolumn{7}{|l|}{ Joint excursion } \\
\hline \multirow{2}{*}{ Elbow excursion (degrees) } & 7 & Light & 0.04 & 0.54 & $\begin{array}{l}32.92(31.70- \\
53.96)\end{array}$ & $\begin{array}{l}35.97(26.62- \\
44.74)\end{array}$ \\
\hline & 8 & Heavy & 0.09 & 0.42 & $\begin{array}{l}42.58(30.40- \\
53.88)\end{array}$ & $\begin{array}{l}40.60(28.88- \\
46.26)\end{array}$ \\
\hline \multirow{4}{*}{$\begin{array}{l}\text { Max. elbow extension angle } \\
\text { (degrees) }\end{array}$} & 8 & Light & 0.01 & -0.63 & 122.26 & 123.67 \\
\hline & & & & & $\begin{array}{l}(109.94- \\
135.51)\end{array}$ & $(111.60-137.16)$ \\
\hline & 8 & Heavy & 0.26 & -0.28 & 124.90 & 125.69 \\
\hline & & & & & $\begin{array}{l}(103.94- \\
135.60)\end{array}$ & $(109.25-137.20)$ \\
\hline \multirow{2}{*}{$\begin{array}{l}\text { Wrist flexion-extension ex- } \\
\text { cursion (degrees) }\end{array}$} & 8 & Light & 0.58 & 0.14 & $\begin{array}{l}12.66(7.49- \\
15.90)\end{array}$ & $11.14(6.39-15.48)$ \\
\hline & 7 & Heavy & 0.40 & 0.23 & $\begin{array}{l}14.97(13.90- \\
17.89)\end{array}$ & $\begin{array}{l}14.95(11.83- \\
18.16)\end{array}$ \\
\hline
\end{tabular}

\section{Discussion}

The influence of grip support from the soft-robotic ironHand glove on movement execution of the upper extremity during a simulated reach-and-grasp task with a light (100 grams) and heavy cylindrical object ( $\geq 1000$ grams) has been investigated in the present study. As expected, no positive influence of the ironHand glove on total movement duration was found in either of the weight conditions. During the light condition, movements were executed within the lower range of a person's capacity. In the heavy condition, movements were performed more towards, but still within, upper limits of functional performance. With glove, transportation of light objects occurred with a lower peak velocity and higher elbow extension, and grasping of the object involved an increased hand opening, while with the heavy objects relative grasp duration was longer and relative transport duration with the object was shorter, compared to without glove.

So far, only very few studies have investigated functional performance with and without 
support from a soft-robotic glove [106-109, 124, 125]. The group of Polygerinos has assessed the direct effect of a soft-robotic glove on the Jebsen-Taylor Hand Function Test in one healthy subject, and reported that it took longer to perform several tasks with glove compared to normative performance times of healthy subjects without glove [125]. Although this finding is in line with previous studies performed with former versions of the current ironHand glove within the elderly population with or without age-related diseases [106-109], the longer time needed to perform a task with glove was not seen in the current study.

Although some studies assessed movement execution during ADL of older adults [118], to our knowledge no study assessed the direct influence of a wearable assistive technology of the hand for older adults on movement execution during a functional task. The light condition was well within the performance range of the subjects. Possibly, it is unnatural to perform a task, which can be performed without support, while wearing a glove that provides unnecessary grasp support and decreases sensation. Therefore, compensation for an unknown situation or decreased sensation because of wearing a glove might have affected the performance in the light condition. Peak velocity of the hand during the task was lower and elbow excursion was larger with glove in the light condition compared to without glove.

On the other hand, when participants had to perform a task closer to the upper limits of their functional capacity, the disadvantageous influence regarding peak velocity was absent. Moreover, transport of the heavy object, the phase in which the glove supports the user most, was faster with glove compared to without glove. This suggests that grasp support can be beneficial for older adults while performing a task close to the limits of their functional capacity. The participants might have felt more confident when using the glove with the heavy object, enabling them to increase their movement speed when holding the object. The high usability score and positive attitude towards the ironHand glove observed in previous studies with the glove [106] might support this improved confidence experienced when grasping and lifting objects.

Although participants needed less time to transport the heavy object with assistance of a soft-robotic glove as compared to without, a relatively longer time was needed to grasp the heavy object with glove support. It is likely that the observed positive effects (relative shorter transporting phase) were counterbalanced by the negative influences (relative longer grasping time), resulting in no differences in total movement time as observed in the present study. This might also play a role in the lack of improved performance time on functional level with the glove, as was found in previous studies [106, 108, 109]. One plausible explanation for the relatively longer grasping phase is that the participants waited for feedback from the system, in the form of noticeable force exerted on their hand, before they felt confident enough to lift 
the object. In this case, the time it takes between registration by the glove's control system that support is needed and actual force exertion being perceived by the participant is represented as a delay during grasping of the object. Therefore, it is beneficial to explore possibilities to reduce the time between grasp initiation and actual force transfer of the glove on the hand. One option is to detect grasping movements before actual contact with the object is made. To realize this, reach must be distinguished from reach-to-grasp movements. Possible ways for exploration of earlier grasp intention detection are with use of electromagnetic sensors, inertial measurement units, bend sensors and pressure sensors [126-128].

A factor that might have contributed to the extent to which effects were (not) observed in the present study, is the time dedicated to familiarization. Although participants used the glove for approximately 10 minutes before starting the reach-and-grasp tasks until they felt comfortable with it, it is possible that they did not reach its full potential [129]. Radder et al.[109] reported that functional performance time of older adults with hand function problems with a previous version of the ironHand glove increased during no more than three repetitions up to the level of unsupported movements, despite an initial slower performance with glove. Nevertheless, in the study of Radder et al.[109] no plateau in performance was reached yet after three repetitions, suggesting that performance time may have improved further beyond the familiarization time applied in the present study. Although so far there are no studies that assessed the effect of prolonged use of an orthosis that supports grip function of older adults, it is known that training is essential to improve the performance of older adults with declined hand function [104]. Therefore, prolonged low-intensity training with an assistive soft-robotic glove in a home setting might enhance hand function of older adults. Additional research, in which the ironHand glove is used for a prolonged period in everyday life, is planned to obtain more insight into its possible effects on functional performance.

A few limitations of this study should be taken into account when interpreting the results and generalization towards activities of daily living. Firstly, this study is only performed in a small group ( $n=8$ ) of participants (only women) with diverse pathologies. Secondly, it might be that participants were not completely familiarized with use of the system (as discussed above). Thirdly, even though we attempted to simulate an ADL task, it is not possible to mimic an ADL situation precisely and still standardize it for research purposes, due to the additional factors, such as cognitive load and environment, influencing task performance. Subsequent testing in a home situation is suggested to assess the effect of the glove on functional outcomes and its impact on everyday life. Fourthly, the task was only partly adapted to the participants' abilities. Although the capacity of participants was considered by adjusting the workspace to their reaching range of motion and selecting the heaviest object they could lift, the personal maximum performance was not precisely tested. 
All in all, when exploring the effect of the wearable soft-robotic ironHand glove on movement execution of elderly, both positive and negative influences of using a soft-robotic glove during a simulated ADL task in terms of movement kinematics were found. In contrast to previous studies, a negative influence on total time needed to perform a task was absent, using either light or heavy objects. In a situation in which an $A D L$, such as holding or carrying tableware, groceries or other weighted household items, is represented (object $\geq 1000$ grams), participants might have adapted their movement execution due to the perceived confidence while wearing the glove. However compensation for an unknown situation or loss of sensation due to wearing a glove may have affected the performance of the reach-and-grasp task that can easily be performed without assistance (object of 100 grams). Longer usage of the glove in ADL might overcome effects which are present due to unfamiliarity with glove usage. The obtained information can be used for improving the design of wearable robots for the hand, and to better understand how these systems can be applied successfully in practice. 



\title{
Quantifying the direct influence of a soft- robotic glove on task performance of the affected upper extremity after stroke
}

Chapter 6

\begin{abstract}
Background: Stroke patients frequently experience upper extremity weakness resulting in deterioration of function in activities of daily living. The wearable soft-robotic ironHand glove is developed to support handgrip strength during daily life. This study aimed to explore the direct influence of the grip-supporting soft-robotic glove on movement execution during functional task performance, as well as, user experience.

Methods: Eleven stroke patients with impaired hand function were included for this crosssectional research. Participants performed a standardized reach and grasp task resembling a common movement in ADL with two objects of different weight (100 grams and $\geq 500$ grams), in three different conditions; without glove, with activated glove, and with non-activated glove. Outcome measures included kinematic movement parameters of the upper arm, maximal pinch strength, the System Usability Scale and a semi-structured interview.

Results: Pinch strength increased significantly when using the glove. Wearing the glove in itself (non-activated glove) did affect movement execution of stroke patients in a negative way, predominantly in terms of slower movements and smaller joint excursion. Alterations in joint excursion were particularly found in the wrist and shoulder, with corresponding larger compensatory movements of the trunk. Active support of the glove had no negative influence on movement performance. Participants were positive about the ease of use of the system, and rated system usability with an average score of 71.6 on the System Usability Scale.
\end{abstract}

Conclusions: Positive effects of the glove were seen on grip strength and participants were positive about its usability. On the other side, wearing the glove in itself negatively influenced movement execution indicating that the design of the grip-supporting soft-robotic glove needs further attention. Considering that the soft-robotic glove is designed to support grip strength, it is plausible that the advantages of the glove become apparent in tasks involving prolonged usage of the hand. So, besides potential design adaptations, future studies should focus on assessing the effect of the glove during tasks involving grip strength and endurance aspects. 


\section{Introduction}

Sophisticated task performance of activities of daily living (ADL) requires proper functioning of the upper extremity [130]. Unfortunately, dysfunction of the motor control system is a frequent and widely recognized impairment after stroke. Upper extremity weakness or hemiparesis is the most prevalent impairment after a first-ever stroke.[7] As a result, stroke patients experience difficulties in independently performing tasks such as drinking, eating, opening buttons of clothing, and writing. This loss in dexterity and corresponding deterioration of $A D L$ often affects functional independence and reduces quality of life.[12, 13] Maximizing the possibilities to use the upper extremity during $A D L$ is important in order to regain or maintain a high level of independence.

Maximal grip force of the affected side often remains reduced after rehabilitation as compared to the non-affected side or healthy subjects [131]. Robotic devices have the potential to support the loss of functionality in upper extremity motor function $[10,20,132,133]$. Studies into the effect of robotic training on the proximal part of the upper extremity have shown predominantly improvements in motor function $[10,20,132,133]$. This therapeutic effect can be, among other things, gained by a variance in assistance, resistance, or movement amplitude [133]. Lambercy et al.[134] for example, used a robotic device that patients could grasp and manipulate by pronation/supination of the forearm while receiving visual feedback. When training the distal part of the upper extremity using robotics promising results were found, and it is suggested by Lambercy et al.[134] that training of solely the distal arm, e.g., the wrist and the hand, may lead to improved function of the entire arm in chronic stroke patients [19, 134, 135]. However, the reported improvements in motor function after robotics training of both the proximal and the distal arm did not transfer to an improved performance of daily activities as quantified by clinical measures [20,132, 134]. Hence, in addition to using therapeutic devices for training purposes of the distal part of the upper extremity, chronic patients could benefit from devices that assist ADL directly. If such devices could be used during ADL at home, functional activities can be supported directly, while at the same time these supported activities might operate as arm-hand function training [136]. Since these activities are part of the daily life of a specific patient, the training is highly intensive and taskspecific, two of the prerequisites for effective rehab training.

An easy-to-use system based on the concept of a wearable robotic glove, called the ironHand, was developed in order to support stroke patients and elderly with dexterity problems during ADL [137]. The grasp support system provides additional grip strength in an intuitive way, but only if the user initiates the movement actively. Only three fingers; the thumb, middle- and ring finger, are covered by the glove to maintain sensation during object manipulation. $A$ study into the feasibility of the wearable soft-robotic ironHand glove in an elderly population 
has shown that a mean System Usability Score of $70 \%$ was achieved [138], which indicates a good probability for acceptance by the users. However, the study also showed that the subjects performed timed tasks significantly slower with the soft-robotic glove as compared to without the glove. A similar effect was found in the study of Prange et al.[139], in which the direct effect of a five-fingered soft-robotic glove was assessed in five chronic stroke patients. Almost all participants performed the Jebsen-Taylor Hand Function Test slower with glove, except for the subtest 'lifting full cans', where performance was faster with glove. Similarly, when the Jebsen-Taylor Hand Function test was performed by a healthy subject when wearing an EMG-triggered wearable soft-robotic glove, a slower performance than expected based on normative data for unsupported healthy individuals was shown [84].

Although the Jebsen-Taylor Hand Function Test has a primary focus on task completion, and therefore assesses the activity levels of the ICF framework, only performance time is assessed while other aspects necessary for task accomplishment, that could be influenced by the glove, are not considered. For that reason it is plausible that potential specific changes in upper extremity function are not captured with such timed outcomes measures (e.g., faster transportation of objects or smoother movements), while those changes could make a difference in functional performance of the hand in daily life $[110,111]$. Therefore, additional kinematic movement analysis is useful as a more extensive and objective assessment to detect differences in motor performance and movement quality on the body function level of the ICF framework [112, 140,141]. Even though several studies quantified upper extremity movements of stoke patients during (functional) tasks [142], research on the effect of a robotic device on movement execution of stroke patients is still scarce [119]. Hence, it is unknown what the effect of a grip-supporting soft-robotic glove, both in activated an non-activated state, is on movement execution after stroke. The goal of this study was therefore to explore the direct influence of the grip-supporting soft-robotic glove on task performance of the affected arm and hand after stroke in a simulated functional setting. Secondary, user experience was assessed.

\section{Methods}

\section{Participants}

Eleven stroke patients (5 male; mean age 62.5 years, standard deviation 8.4) were recruited for this cross-sectional study performed at the movement analysis lab of Roessingh Research and Development, Enschede, the Netherlands. Participants met the following inclusion criteria: 1) clinically diagnosed with unilateral ischemic or hemorrhagic stroke at least three months before inclusion; 2) between 18-80 years old; 3) able to perform 10 degrees of active flexion and extension of the fingers; and 4) a sufficient cognitive status to understand two-step 
instruction in Dutch. Criteria for exclusion were severe sensory problems of the affected hand and arm, severe acute pain of the affected hand, severe contractures limiting the passive range of motion and co-morbidities limiting functional use of the hand. This study was approved by the Medical Ethical Committee Twente, the Netherlands (number NTR6169). Participants gave written informed consent prior to participation to this study.

\section{Soft-robotic glove}

The ironHand glove is a wearable soft-robotic glove consisting of a three-fingered glove $( \pm$ 85 grams) and a control unit ( \pm 600 grams) that contains the embedded software to control the amount of force needed to support grip strength, and the batteries [137]. Grip strength is supported by the glove via the thumb, middle- and ring finger. The amount of support is regulated by a tendon-driven mechanism receiving input from pressure sensors located at the fingertips of the ironHand glove, and bend sensors situated along the dorsal side of the fingers. The grip strength was limited at $20 \mathrm{~N}$ for safety. The amount of support was set on a level matching the participants' needs and experienced comfort. In order to further improve wearing comfort and optimal control of sensor activation, the glove was available in three different sizes (small, medium, and large), allowing participants to use the appropriate size during this research.

\section{Study design}

The experiment started with the performance of the upper extremity part of the Fugl-Meyer assessment (FMA), without reflexes, in order to determine the current upper extremity function, without any support [88]. Next, participants familiarized themselves with the soft-robotic glove prior to the start of the measurements for at least 20 minutes. All measurements were performed with the affected side. Pinch strength and movement execution during a functional task were assessed with and without wearing the glove. The functional task was performed once without and twice with the soft-robotic glove (once with and once without actuation of the system). The order of glove condition for all tests was randomized beforehand with a custom-made Matlab script. Perceived usability was assessed at the end of the measurement by means of the System Usability Scale (SUS) [143] and a semi-structured interview.

\section{Pinch strength}

Maximal pinch strength between thumb and index finger, and thumb and middle finger of the affected hand was measured with the Baseline ${ }^{\circledR}$ Lite $^{\mathrm{TM}}$ Hydraulic Pinch Gauge dynamometer (Fabrication Enterprises, White Plains, New York, USA). The participant squeezed the dynamometer maximally with the distal segment and ventral side of the thumb, and index finger or middle finger, which was repeated three times. Between the attempts there was a period of rest of at least 60 seconds. The average of the three scores was calculated. 


\section{Movement execution during functional task}

Movement execution was assessed during a standardized task representing a common movement in $\mathrm{ADL}$, including reaching to, grasping of, transportation of, and releasing of an object (Figure 6.1). The participants were shown and verbally instructed on how to perform the task. Participants sat in front of a height adjustable table with the affected upper arm aligned to the trunk, the elbow flexed 90 degrees and the hand positioned neutrally at a predefined start location. Participants were asked to place a cylindrical object with a diameter of $5 \mathrm{~cm}$, positioned at a predefined position in front of the hand, on a platform. The platform was located $35 \mathrm{~cm}$ in front of the participant, and was aligned with the midline of the trunk. The height of the platform was adjusted to $5 \mathrm{~cm}$ below the shoulder height of the participant. All participants were able to comfortably place the cylindrical object on the platform. Five different phases of the task were defined: (1) reaching to the cylindrical object; (2) grasping the object; (3) transporting the object to the platform; (4) releasing the object at the platform; and (5) reaching to the initial start location. The task was performed with two different weights of the cylindrical object: a light weight condition of 100 grams and a heavy weight condition of either 500,1000 or 2500 grams depending on the capability of the participant to lift a heavy object onto the platform, while receiving support from the soft-robotic glove. The light condition was included to assess the effect on movement execution without the weight of the object interfering with the movement execution. The heavy condition was included to simulate an ADL task, in which usually weight has to be lifted. The weight used for the heavy condition was determined prior to the start of the reach-and-grasp task based on the participant's ability to lift the heaviest weight possible onto the platform while receiving support from the glove. The reach-and-grasp task was then performed fifteen times for each weight: five times without glove (glove off), five times with the non-activated glove (glove on - support off, and five times with glove support (glove on - support on). The order of the weight condition was randomized, as well as the order of glove condition.
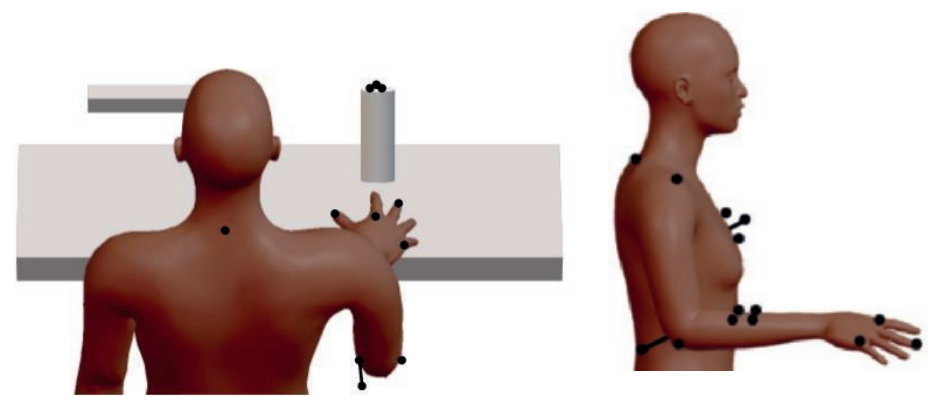

Figure 6.1 Left: Schematic measurement set-up with black dots indicating marker position. Right: Lateral view of participant with black dots indicating marker position. 
Upper extremity movements during the functional task were registered with the motion capture system VICON MX13+ (Oxford Metrics, Oxford, UK). Six infrared cameras recorded the position of the fifteen reflective markers as used in this study. Positioning of these reflective markers on the hand, arm, thorax and neck was equal to the set-up as used in the study with elderly by van Ommeren et al.[144], and was based on the guidelines of the International Society of Biomechanics [116]. Three additional markers were placed on the cylindrical object to record its movements during the task.

\section{User experience}

Perceived usability of the soft-robotic glove was measured with the SUS. The SUS contains ten items that can be scored on a five-point Likert scale ranging from 'strongly agree' to 'strongly disagree'. Higher scores (maximum 100), indicate a better usability. [143] Scores above 90 represent exceptional usability and scores between 50 and 70 represent promising usability, while a score below 50 indicates a high likelihood that the product will face usability difficulties in the field $[89,91]$. Additionally, a semi-structured interview provided additional insight into patients' opinions on usability and experience, and identified positive aspects and points for improvement of the system. The interview included the following four questions: (1) What is your opinion about the ease of use of the glove and the support it provides?; (2) What is your experience with donning and doffing the system?; (3) Overall, what were the best aspects of this prototype?; (4) What aspects of this prototype could be improved?

\section{Data analysis}

VICON nexus 1.8.2 was used to pre-process the recorded upper extremity movement data. The pre-processed data was transferred to MATLAB software (R2015a, MathWorks Inc., Natick, Massachusetts, USA) for further custom off-line analysis. A low pass filter with a second order Butterworth filter with a cut-off frequency of $20 \mathrm{~Hz}$ and zero phase shift was used to reduce noise levels.

\section{Segmentation}

The same set-up was used as in the study of Van Ommeren et al.[144]; the task was divided into five phases as described below and represented in Figure 6.2. In order to facilitate the determination of the five movement phases, indices were chosen beforehand at $15 \%$ of the maximal velocity of the hand, based on the second metacarpal marker (Figure 6.2).

1. Reach-to-grasp. The reach-to-grasp phase started at first moment that the tangential velocity of the hand exceeded $2 \%$ of the maximum velocity of the hand based on the second metacarpal marker [118]. The end of the reach-to-grasp phase was based on either the position of the cylindrical object relative to the hand, or the velocity profile 
of the cylindrical object within the timeframe between index 2 and index 3 (Figure 6.2). The difference in position of the cylindrical object relative to the hand reaches a minimum when an attempt is made to grasp. Additionally, it is assumed that the cylindrical object was moved by an external source if its velocity was for the first time larger than the mean plus two times the standard deviation of when the cylindrical object stood still. The end of the first reach was set at the lowest frame number of the two options.

2. Grasp. The start of this phase coincided with the end of the reach-to-grasp phase and the end of grasping was defined as the last frame number of either;

i. the difference in velocity of the cylindrical object relative to the hand marker was smaller than the mean value minus two times the standard deviation of the velocity of the cylindrical object, as measured during the reach-to-grasp phase. During the reach-with-object phase, the hand and cylinder were expected to have the same movement pattern. The difference in velocity of the hand and cylinder will therefore be minimal;

ii. the latest minimum of the vertical position of the cylindrical object in time between index 2 and index 3 (Figures 6.2). Before the object was lifted in vertical direction, a minimum was seen in the vertical position profile of the cylindrical object;

iii. the combined $X Y Z$-position of the cylindrical object is larger than two times the standard deviation plus the mean combined $X Y Z$ value of the cylindrical object when not moved and if fifteen frames later the XYZ position of the cylindrical object is more than twelve times the standard deviation plus mean of the cylindrical object when not moved. If grasped, the object's movement was not necessarily in the vertical direction. In the case that the object was moved because of touching instead of measuring the end of grasping, the condition that the object had to be moved substantially (ten times the standard deviation (SD) + mean) was built in.

The three criteria were needed to cover the possible situations to define the grasp phase.

3. Reach-with-object. The start of this phase coincided with the end of the grasp phase. The end of this phase was set at the frame number when the first minimum value of the vertical position of the cylindrical object occurred, after the maximum vertical peak value in vertical position of the cylindrical object. This event was chosen because the object was always lifted higher than the height of the platform (maximum in vertical position), after which the impact of the cylindrical object with the platform caused a minimum in the vertical position of the cylindrical object.

4. Release. This phase started at the end of the reach-with-object phase and ended with the last frame number of either; 
i. the first time that the difference in position of the cylindrical object and hand marker exceeded the minimal distance, as determined in the reachto-grasp phase, between cylindrical object and hand plus 15 times the standard deviation;

ii. the last time that the velocity of the cylindrical object exceeded the mean velocity plus two times standard deviation of the cylindrical object as measured in rest.

5. Final reach. The start of the final reach coincided with the end of the release phase and the end of this phase occurred at the first time that the tangential velocity of the hand was smaller than $2 \%$ of the maximum speed of the hand [118].

The grasp and release phases were manually checked by comparing the frame numbers to visual observation of VICON data. If the algorithm and manual check deviated more than 0.05 $\mathrm{s}$, parameters associated with that grasp or release phase were removed from further analysis.

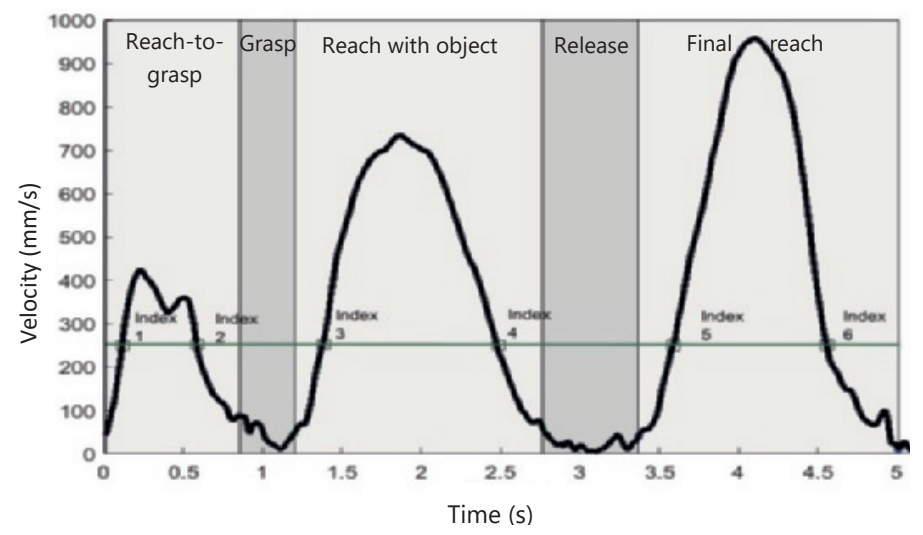

Figure 6.2 Division of tangential velocity profile of the hand marker in five phases. The horizontal line represents a threshold used for the detection of the five phases of the reach and grasp task (schematic representation).

\section{Kinematics}

Kinematic variables were calculated from the 3D upper extremity position data as measured during the task representing a movement common in ADL. The time needed to execute each of the five phases was calculated. Movement smoothness, defined by the number of movement units (NMU), was calculated during the movement. To define a NMU, local minima and maxima in the tangential velocity profile of the marker on the second metacarpal head were searched. The tangential velocity profile was calculated as: $V_{T}=\sqrt{V_{x}^{2}+V_{y}^{2}+V_{z}^{2}}$, with $V_{x^{\prime}} V_{y^{\prime}}$ and $V_{z}$ calculated by numerical differentiation of $x, y$, and $z$ projections of the spatial trajectories of the marker on the second metacarpal head [145]. The difference between a consecutive 
minimum and maximum with an amplitude of $20 \mathrm{~mm} / \mathrm{s}$ or more, indicated a velocity peak that corresponds to the smoothness and efficiency of movement [121]. If the time between two consecutive peaks exceeded 150 milliseconds, a movement unit was detected [118].

The peak tangential velocity of the hand (second metacarpal marker) was calculated as the maximal speed measured during the movement. Maximal hand opening during the reachto-grasp phase was determined as the maximal 3D distance between the thumb and middle finger marker.

Maximal trunk displacement was defined as the maximal 3D displacement of the trunk marker during the task when compared to the initial position in rest. Joint excursion was calculated from the 3D position data for the shoulder (plane of elevation, elevation angle, and internal or external rotation angle), the elbow and the wrist (flexion/extension, internal/external rotation and deviation). The ISB recommendations were used to calculate the shoulder and the wrist angles [116]. Shoulder excursion was calculated by subtracting the smallest angle between the humerus and thorax from the largest angle during the task in each of the three directions. The elbow angle during the entire execution of the task was calculated from the $3 \mathrm{D}$ joint angle between the vectors of the upper- and lower arm. Elbow excursion within the performed task was determined by subtraction of the smallest angle between those vectors from the largest angle. Maximum elbow extension angle was represented by the largest angle between the upper- and lower arm. Wrist excursion was calculated by subtracting the smallest angle between the forearm and hand from the largest angle during the task in each of the three directions (flexion/extension, radial/ulnar deviation, and pronation/supination). Due to cross talk between wrist flexion/extension and abduction/adduction movements caused by the choice in segment orientation and joint coordinate systems, wrist abduction/adduction excursion was omitted from the analysis.

\section{Statistical analysis}

Individual scores across participants were averaged per glove condition and weight of the cylindrical object. The IBM SPSS Statistics version 23.0 package for Windows was used to analyze the data. Prior to selection of appropriate statistical tests, normal distribution of all outcome measures was inspected using Q-Q plots and the Shapiro-Wilk test. Group scores per parameter were reported as mean with standard deviation, or median with interquartile range for non-parametric outcomes. Movement parameters were analyzed using mixed model repeated measures analysis or the non-parametric equivalent, Friedman's ANOVA. In case of significance for non-parametric outcome measures, additional Wilcoxon Signed Rank tests were performed. Adjustment for multiple comparisons was performed with the HolmBonferroni method, or the Benjamini-Hochberg method (false positive rate of $10 \%$ ) in case of 
non-parametric testing. Pinch strength parameters were analyzed by a paired samples t-test. Effects of all parameters were considered statistically significant if $\alpha<0.05$.

\section{Results}

\section{Participants}

Eleven participants were included in the study. The patient characteristics are displayed in Table 6.1. One participant could not grasp the cylindrical object properly while using the softrobotic glove since active hand opening was highly impaired. Due to limited active extension of the fingers, the patient tended to push the fingers around the cylindrical object. While wearing the glove, the pressure sensors got activated, and therefore support was already provided, before the fingers were completely around the object. Therefore, no movement execution data is available from this participant (FMA = 33). The group involved five (45\%) mildly affected (FMA score > 41), and six (55\%) moderately affected (28 $\leq$ FMA score $\geq 41$ ) stroke patients, based on a categorization of the FMA score [92]. Five participants had right hemiparesis and six had left hemiparesis. The affected side was the dominant side pre-stroke in six participants.

Table 6.1 Participant characteristics.

\begin{tabular}{|c|c|}
\hline & Participants $(\mathrm{N}=11)$ \\
\hline Sex (male/female) ${ }^{a}$ & $5 / 6$ \\
\hline Age (years) ${ }^{b}$ & $62.5 \pm 8.4(51-77)$ \\
\hline Time post stroke (years) ${ }^{b}$ & $5.7 \pm 2.2(2-9)$ \\
\hline Affected body side (right/left) ${ }^{a}$ & $5 / 6$ \\
\hline Dominant body side pre-stroke (right/left) ${ }^{\mathrm{a}}$ & $10 / 1$ \\
\hline Fugl-Meyer assessment score (range $0-66$ points) ${ }^{b}$ & $48.3 \pm 8.4(37-61)$ \\
\hline
\end{tabular}

${ }^{\text {a }}$ Absolute numbers, ${ }^{\mathrm{b}}$ mean \pm standard deviation (range)

\section{Pinch strength}

Pinch strength between thumb and middle finger improved on average by $28 \%(t(5)=-3.216$, $p=0.02)$ when receiving support from the glove $(4.62 \mathrm{kgs} \pm 1.27 \mathrm{kgs})$ with respect to glove off (3.75 kgs $\pm 1.34 \mathrm{kgs}$ ). Although not statistically significant, pinch strength between thumb and index finger increased on average 15\% when receiving support from glove on- support on as compared to glove off $(\mathrm{t}(10)=-2.082, \mathrm{p}=0.06)$.

\section{Movement execution}

When comparing kinematic parameters between glove on - support on and glove off 
conditions, a direct, but negative effect on movements execution was observed (Table 6.2). Duration of the reach-with-object phase with the light object was longer $(p=0.02)$, trunk displacement larger $(p=0.02)$ and wrist flexion/extension excursion smaller $(p=0.02)$ with glove on - support on compared to glove off. With the heavy object, total movement time was longer $(p<0.01)$, grasp duration was longer $(p<0.01)$, peak velocity of the hand was lower $(p=0.03)$, shoulder elevation excursion was smaller $(p<0.01)$ and wrist flexion/extension excursion was smaller $(p<0.01)$ with glove on - support on compared with glove off.

\section{Single effect of glove actuation}

When assessing the sole effect of glove support by comparing task performance between glove on- support off and glove on - support on, the only difference was observed with the light object. In line with the comparison of glove on - support on with glove off conditions, the reach-with-object phase was longer when glove on - support on was compared to glove on- support off (Table 6.2, $p<0.05$ ). No other significant differences in temporal variables and movement kinematics were found when comparing movement execution were found.

\section{Single effect of wearing the glove}

When assessing the influence of just having the glove on by comparing movement execution between glove on - support off and glove off conditions, remarkable differences were observed (Table 6.2). For the light object, duration of the reach-with-object phase was longer when wearing the glove $(p=0.02)$, and peak velocity of the hand was higher $(p=0.03)$ in glove on - support off condition compared to glove off. In addition, trunk displacement was larger $(p<0.05)$, wrist flexion/extension excursion was smaller $(p<0.05)$, excursion in shoulder elevation angle was larger $(p=0.03)$, and maximum elbow extension angle was smaller $(p=$ 0.03 ) with glove on - support off.

For the heavy object (Table 6.2), total time needed to perform the task, and time needed to grasp the object was larger ( $p<0.01$ and $p<0.01$, respectively), while peak velocity of the hand and wrist pronation/supination excursion were smaller $(p=0.03)$ with glove on support off when compared with glove off (Table 6.2). Wrist flexion and extension excursion was smaller with glove on - support off when performing the task with both the light and heavy object, as compared with glove off.

\section{User experience}

User experience as measured with the SUS (Figure 6.3) showed a mean score of 71.6 (SD = 19.6). On an individual level, six participants rated usability over 70 , three participants between 50 and 70 and two below 50 . 


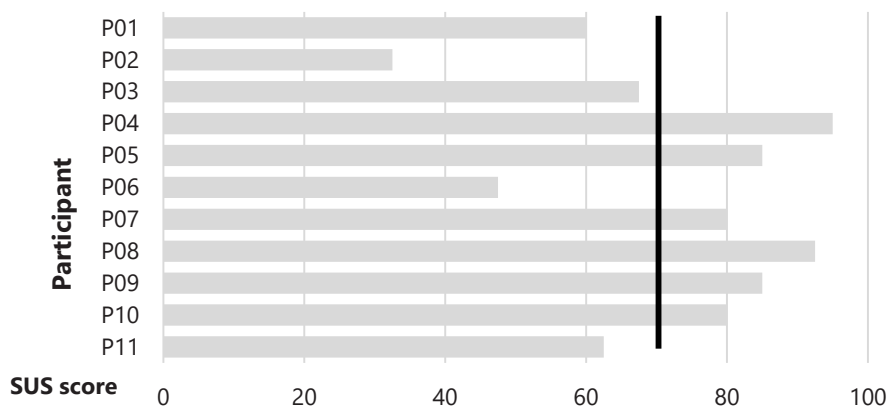

Figure 6.3 SUS scores presented per participant. The black line represents the mean SUS score.

According to the answers on the semi-structured interviews, most of the participants (82\%) explained that it was easy to use the soft-robotic glove. One of the participants made a remark that it became easier while using it longer, and another participant explained that he felt more strength but experienced less freedom of movement. With respect to donning and doffing of the glove, the participants with relatively better hand function, based on the FMA, did not experience any problems, whereas the relatively more affected patients reported more often that they struggled to get their fingers into the right glove compartment. As for the best aspects of this prototype, several participants (30\%) appreciated the glove having two glove-free fingers, which helped to maintain sensation during object manipulation. It was mentioned by seven participants that the experience that the glove actually supports grip strength increased their perceived grip assurance and confidence when moving. The points for improvement that were mentioned by participants include the limitation in freedom of movement (27\%), the glove being not water-resistant (27\%), and the loss of sensation in the finger tips combined with the type of fabric that makes smaller objects slip away (36\%). 
Table 6.2 Outcome values without glove, with glove - actuated, and with glove - non actuated for light and heavy condition.

\begin{tabular}{|c|c|c|c|c|c|c|c|c|c|}
\hline & & & & & & & \multicolumn{3}{|c|}{ Post-hoc p value } \\
\hline $\begin{array}{l}\text { Outcome } \\
\text { measure }\end{array}$ & p value & Weight & $\mathbf{N}$ & $\begin{array}{l}\text { Glove off } \\
\text { (without) }\end{array}$ & $\begin{array}{c}\text { Glove } \\
\text { on - } \\
\text { support } \\
\text { on } \\
\text { (withA) }\end{array}$ & $\begin{array}{c}\text { Glove } \\
\text { on - } \\
\text { support } \\
\text { off } \\
\text { (withN) }\end{array}$ & $\begin{array}{c}\Delta_{\text {withA- }} \\
\text { without }\end{array}$ & $\begin{array}{c}\Delta_{\text {withN- }} \\
\text { without }\end{array}$ & $\underset{\text { withA }}{\Delta_{\text {withN }}}$ \\
\hline \multirow{2}{*}{ Total time (s) } & $0.061^{b}$ & Light & 10 & $3.4(2.5)$ & $4.1(0.5)$ & $3.7(1.9)$ & & & \\
\hline & $0.014^{b}$ & Heavy & 10 & $4.1(1.4)$ & $4.9(1.2)$ & $4.4(1.8)$ & 0.009 & 0.013 & 0.386 \\
\hline \multirow{2}{*}{$\begin{array}{l}\text { Time reach- } \\
\text { to-grasp } \\
\text { phase (s) }\end{array}$} & $0.082^{b}$ & Light & 10 & $0.6(0.4)$ & $0.6(0.4)$ & $0.8(0.2)$ & & & \\
\hline & $0.213^{a}$ & Heavy & 10 & $0.7(0.2)$ & $0.8(0.4)$ & $0.7(0.2)$ & & & \\
\hline \multirow{2}{*}{$\begin{array}{l}\text { Time grasping } \\
\text { (s) }\end{array}$} & $0.497^{b}$ & Light & 10 & $0.2(0.3)$ & $0.2(0.4)$ & $0.3(0.3)$ & & & \\
\hline & $0.002^{b}$ & Heavy & 10 & $0.2(0.3)$ & $0.4(0.4)$ & $0.3(0.4)$ & 0.009 & 0.005 & 0.878 \\
\hline \multirow{2}{*}{$\begin{array}{l}\text { Time reach- } \\
\text { with-object } \\
\text { (s) }\end{array}$} & $0.014^{b}$ & Light & 10 & $1.0(0.2)$ & $1.2(0.3)$ & $1.1(0.4)$ & 0.022 & 0.047 & 0.047 \\
\hline & $0.061^{\mathrm{a}}$ & Heavy & 10 & $1.2(0.2)$ & $1.3(0.2)$ & $1.3(0.2)$ & & & \\
\hline \multirow{2}{*}{$\begin{array}{l}\text { Time release } \\
\text { (s) }\end{array}$} & $0.071^{\mathrm{b}}$ & Light & 10 & $0.4(0.8)$ & $0.4(0.7)$ & $1.3(0.8)$ & & & \\
\hline & $0.55^{\mathrm{a}}$ & Heavy & 10 & $0.8(0.3)$ & $0.9(0.3)$ & $0.7(0.2)$ & & & \\
\hline \multirow{2}{*}{$\begin{array}{l}\text { Time to base } \\
\text { (s) }\end{array}$} & $0.15^{\mathbf{b}}$ & Light & 10 & $1.1(0.5)$ & $1.3(0.6)$ & $1.3(0.5)$ & & & \\
\hline & $0.497^{b}$ & Heavy & 10 & $1.2(0.7)$ & $1.4(0.6)$ & $1.5(1.0)$ & & & \\
\hline \multirow{2}{*}{$\begin{array}{l}\text { Peak velocity } \\
(\mathrm{m} / \mathrm{s})\end{array}$} & $0.007^{a}$ & Light & 10 & $1.0(0.2)$ & $0.9(0.2)$ & $0.9(0.2)$ & 0.073 & 0.032 & 1.000 \\
\hline & $0.002^{a}$ & Heavy & 10 & $0.9(0.1)$ & $0.8(0.2)$ & $0.8(0.1)$ & 0.031 & 0.001 & 1.000 \\
\hline \multirow{2}{*}{$\begin{array}{l}\text { Movement } \\
\text { smoothness } \\
\text { (NMU) }\end{array}$} & $0.67^{\mathbf{b}}$ & Light & 10 & $7.2(8.0)$ & $9.2(8.1)$ & $7.8(6.2)$ & & & \\
\hline & $0.355^{\mathrm{a}}$ & Heavy & 10 & $11.6(4.0)$ & $12.3(3.9)$ & $12.0(4.5)$ & & & \\
\hline \multirow{2}{*}{$\begin{array}{l}\text { Maximum } \\
\text { hand opening } \\
(\mathrm{cm})\end{array}$} & $0.686^{a}$ & Light & 10 & $15.1(2.0)$ & $15.4(1.6)$ & $15.2(1.6)$ & & & \\
\hline & $0.355^{\mathrm{a}}$ & Heavy & 10 & $15.0(2.2)$ & $14.5(1.7)$ & $15.0(1.7)$ & & & \\
\hline \multirow{2}{*}{$\begin{array}{l}\text { Trunk } \\
\text { displacement } \\
\text { (cm) }\end{array}$} & $0.007^{b}$ & Light & 10 & $2.8(14.9)$ & $8.5(12.4)$ & $7.3(11.4)$ & 0.022 & 0.047 & 0.139 \\
\hline & $0.469^{a}$ & Heavy & 10 & $12.0(6.3)$ & $13.7(6.9)$ & $13.1(6.9)$ & & & \\
\hline \multirow{2}{*}{$\begin{array}{l}\text { Shoulder } \\
\text { plane of } \\
\text { elevation } \\
\text { excursion } \\
\text { (deg) }\end{array}$} & $0.492^{\mathrm{a}}$ & Light & 10 & $59.4(14.3)$ & $56.6(7.3)$ & $56.6(7.3)$ & & & \\
\hline & $0.005^{a}$ & Heavy & 10 & $63.4(13.0)$ & $\begin{array}{c}54.9 \\
(13.2) \\
\end{array}$ & $\begin{array}{c}57.2 \\
(14.5) \\
\end{array}$ & 0.003 & 0.114 & 1.000 \\
\hline \multirow{2}{*}{$\begin{array}{l}\text { Shoulder } \\
\text { elevation } \\
\text { excursion } \\
\text { (deg) }\end{array}$} & $0.025^{b}$ & Light & 10 & $58.9(11.7)$ & $\begin{array}{c}57.5 \\
(12.0 .)\end{array}$ & $\begin{array}{c}57.5 \\
(11.5)\end{array}$ & 0.074 & 0.037 & 0.878 \\
\hline & $0.905^{b}$ & Heavy & 10 & $57.1(17.0)$ & $\begin{array}{c}61.1 \\
(10.8)\end{array}$ & $\begin{array}{c}61.2 \\
(11.8)\end{array}$ & & & \\
\hline
\end{tabular}


Table 6.2 Outcome values without glove, with glove - actuated, and with glove - non actuated for light and heavy condition. (continued)

\begin{tabular}{|c|c|c|c|c|c|c|c|c|c|}
\hline & & & & & & & \multicolumn{3}{|c|}{ Post-hoc $p$ value } \\
\hline $\begin{array}{l}\text { Outcome } \\
\text { measure }\end{array}$ & p value & Weight & $\mathbf{N}$ & $\begin{array}{l}\text { Glove off } \\
\text { (without) }\end{array}$ & $\begin{array}{l}\text { Glove } \\
\text { on - } \\
\text { support } \\
\text { on } \\
\text { (withA) }\end{array}$ & $\begin{array}{l}\text { Glove } \\
\text { on - } \\
\text { support } \\
\text { off } \\
\text { (withN) }\end{array}$ & $\begin{array}{l}\Delta_{\text {withA- }} \\
\text { without }\end{array}$ & $\begin{array}{l}\Delta_{\text {withN- }} \\
\text { without }\end{array}$ & $\begin{array}{c}\Delta_{\text {withN- }} \\
\text { withA }\end{array}$ \\
\hline \multirow{2}{*}{$\begin{array}{l}\text { Shoulder } \\
\text { axial rotation } \\
\text { excursion } \\
\text { (deg) }\end{array}$} & $0.261^{a}$ & Light & 10 & $56.9(8.5)$ & $\begin{array}{c}61.0 \\
(11.6)\end{array}$ & $55.1(8.8)$ & & & \\
\hline & $0.043^{a}$ & Heavy & 10 & $60.6(9.2)$ & $\begin{array}{c}53.2 \\
(12.2)\end{array}$ & $\begin{array}{c}54.9 \\
(13.6)\end{array}$ & 0.122 & 0.346 & 1.000 \\
\hline \multirow{2}{*}{$\begin{array}{l}\text { Maximum } \\
\text { elbow } \\
\text { extension } \\
\text { (deg) }\end{array}$} & $0.027^{a}$ & Light & 10 & $62.3(14.1)$ & $\begin{array}{c}59.6 \\
(11.9)\end{array}$ & $\begin{array}{c}58.8 \\
(11.7)\end{array}$ & 0.213 & 0.036 & 0.51 \\
\hline & $0.525^{a}$ & Heavy & 10 & $62.8(11.3)$ & $\begin{array}{c}61.6 \\
(10.1)\end{array}$ & $\begin{array}{c}61.0 \\
(10.6)\end{array}$ & & & \\
\hline \multirow{2}{*}{$\begin{array}{l}\text { Elbow } \\
\text { excursion } \\
\text { (deg) }\end{array}$} & $0.569^{a}$ & Light & 10 & $42.6(13.1)$ & $\begin{array}{c}42.8 \\
(11.0)\end{array}$ & $\begin{array}{c}44.0 \\
(13.1)\end{array}$ & & & \\
\hline & $0.441^{a}$ & Heavy & 10 & $42.8(11.0)$ & $\begin{array}{c}44.0 \\
(13.1)\end{array}$ & $\begin{array}{c}42.0 \\
(12.4)\end{array}$ & & & \\
\hline \multirow{2}{*}{$\begin{array}{l}\text { Wrist flexion/ } \\
\text { extension } \\
\text { excursion } \\
\text { (deg) }\end{array}$} & $<0.001^{a}$ & Light & 10 & $47.3(13.3)$ & $\begin{array}{c}35.8 \\
(10.2)\end{array}$ & $\begin{array}{c}34.0 \\
(10.1)\end{array}$ & 0.004 & 0.001 & 0.447 \\
\hline & $<0.001^{a}$ & Heavy & 10 & $50.9(13.9)$ & 37.7 (9.9) & $\begin{array}{c}37.4 \\
(10.2)\end{array}$ & 0.006 & 0.012 & 1.000 \\
\hline \multirow{2}{*}{$\begin{array}{l}\text { Wrist } \\
\text { pronation/ } \\
\text { supination } \\
\text { excursion } \\
\text { (deg) }\end{array}$} & $0.012^{a}$ & Light & 10 & $48.3(13.9)$ & $40.8(6.2)$ & $39.9(6.9)$ & 0.110 & 0.076 & 1.000 \\
\hline & $0.043^{a}$ & Heavy & 10 & $54.3(14.2)$ & $44.2(7.7)$ & 40.4 (7.6) & 0.129 & 0.028 & 0.864 \\
\hline \multicolumn{10}{|c|}{$\begin{array}{l}\text { Mean or median values without glove, with actuated glove, and with non-actuated glove are } \\
\text { displayed for the light and heavy condition seperately. P-values of the main effect are displayed } \\
\text { in the first column and in case of significance, P-values of post-hoc analysis can be found in the } \\
\text { last three columns (significant P-values after adjustment for mutiple testing are displayed in bold). } \\
\text { a = normally distributed variables are displayed by mean (standard deviation) and analyzed by repeated measures } \\
\text { analysis. } \\
\text { b = non-parametric variables are displayed by median (interquartile range) and analyzed by the Friedman test. }\end{array}$} \\
\hline
\end{tabular}

\section{Discussion}

The aim of the present study was to explore the direct, assistive influence of a wearable softrobotic glove on pinch strength and movement execution in stroke patients, while performing a reach-and-grasp task representing a daily life task. Moreover, we assessed differences between task performance with glove off, glove on - support on, and glove on - support off, to obtain insight in the single effects of glove actuation and wearing the glove in itself. Wearing the glove in itself (glove on - support off) did affect movement execution of stroke patients in a negative way, predominantly in terms of slower movements and smaller joint 
excursion. Alterations in joint excursion were particularly found in the wrist and shoulder, with corresponding larger compensatory movements of the trunk. In contrast to just having the glove on, activating the glove itself (glove on - support on) did not negatively affect movement performance as compared to using the non-activated glove (glove on - support off). In addition, pinch strength of stroke patients increased $>15 \%$ when using the activated glove compared to glove-off.

Pinch strength, as measured between thumb and middle finger, was, on average, $28 \%$ larger with use of the soft-robotic glove. Furthermore, although not significant, pinch force between thumb and index finger also increased on average with 15\%. These gains in pinch force are in line with previous research by Radder et al.[108], who found an average increase of pinch strength of $22 \%$ when compared with the ironHand glove in an elderly population. The fact that the glove does not support the index finger in strength could be the reason for a slightly smaller improvement in the current study since stroke patients are most likely unable to match the increased force, coming from their supported thumb, with their unsupported index finger. Although pinch strength between thumb and index finger is expected to be enhanced when the index finger is supported by the glove too, participants appreciate that the index finger is not covered by the glove, since it maintains tactile information, which is found to be important for maintaining a secure grip while manipulating objects [146].

Time needed to complete a simulated ADL task is longer when performed with either an activated or non-activated glove as compared to glove off. Those findings are in line with previous research from Radder et al.[138] and Polygerinos et al.[84], in which the time needed to perform functional tasks and the the Jebsen-Taylor Hand Function Test respectively was longer with glove. However, our study additionally assessed the transparency of the softrobotic glove. Transparency refers to how the device alters free movements [147]. Transparency is seen as a key feature for a robotic device to have to provide valuable assistance [148, 149]. Except for the reach-with-object phase with the light object, no difference in performance was found between movement execution with the activated glove and the non-activated glove. So although movement execution was affected by the glove, it seems that this is not due to the support coming from the glove but due to wearing the glove in itself. As expected, longer time needed to complete the reach-and-grasp task was found during the condition with the light weighted object. It is expected that this has to do with patients waiting for perceiving actual support of the system before moving the object. This can be explained by the fact that there is a certain delay between sensing pressure at the fingertips, activation of the system, and feedback in the form of actual support and this being perceived by the participant.

The most pronounced alteration in joint rotations were found in the wrist flexion/extension 
and pronation/supination excursion angles (over 10 degrees) when wearing the glove during lifting both the light and heavy objects (over 10 degrees). Even though patients are clearly hindered in their freedom of movement, the resulting excursion values are still within the ranges necessary to execute the movement successfully. The joint excursions observed during the current reach-and-grasp task are comparable to those reported for a similar task in which a can was lifted from a shelf [150]. Since the design of the glove includes a relatively large Velcro strap around the wrist to secure the glove on the hand during actuation, the glove may provide more stability to the wrist, which could be beneficial in certain patients with limited active wrist function. At the same time, it is likely that this limits their degrees of freedom. Nevertheless, the extent of the reduction in wrist ROM does suggest that potential design adaptations should be considered. For example, Polygerinos et al.[125] did report an accurate and comfortable range of motion of the hand and wrist when using their glove compared to free movement. Their soft robotic glove has two smaller Velcro's around the wrist instead of one relatively big Velcro as used in the current version of the glove in the present study.

Wearing the glove in itself also affected movements of joints remote from the glove itself. Maximal extension of the elbow and shoulder elevation excursions were smaller with glove on - support off compared to movements with glove off. Trunk displacement increased with glove on - support off to compensate for this. Compensational strategies are often used by stroke patients in order to execute a reach-and-grasp task, to overcome reductions in motor function/movement ability [151]. Although the differences in joint angles are small in the present study, these compensational strategies could be related to the weight of the system. The study of Nijenhuis et al.[119] involved a training device to support wrist extension for home use, for which an arm support device was suggested to add to compensate for the additional weight of the wrist and hand orthoses used in that study. However, for use during $A D L$ at home as intended with the soft-robotic ironHand glove, it is not desirable to have a fixed support system to compensate for the weight of the system. Although the current version is already light in weight, a potential solution would be to further reduce the weight of the part of the ironHand system that is worn on the hand and forearm. Especially the stiffness and weight of the cable, but also the weight or positioning of the connection between cable and glove could be improved.

Previous research by Radder et al.[109] demonstrated a steep learning curve in elderly who used the ironHand system for a short amount of time. Timed functional performance increased across no more than 3 repetitions, within 20 minutes, of using the glove. This might indicate that a longer familiarization time with the glove could result in stabilizing the quality of movement execution to a point that users actually know how to use the glove and perform at their maximum ability. Even though participants used the glove at least 20 minutes before the 
start of the reach-and-grasp tasks in the current study, it could be that some participants were already familiarized while others were not finished learning how to use the glove optimally. Although participants were given the time to familiarize as long as they desired, there could be a discrepancy between perceived performance and objectively measured performance.

The negative influence of wearing the glove on movement execution could also be caused by participants feeling less confident due to a reduced sensation they experienced because of the glove's textile covering larger parts of the ventral side of the fingers and palm of the hand. In general participants were satisfied with usability, represented by a mean score of $71.6 \%$ on the SUS on group level (ranging from 32.5 to 95), indicating a good probability for acceptance by users [91]. Based on the positive usability scores, it could be that patients have made a trade-off between the gain in pinch strength and the slightly slower and smaller movements. In addition to the promising SUS scores, all participants were able to don and doff the glove by themselves. Participants who were less enthusiastic were mainly disappointed by the inability to grasp smaller objects. Since the glove is currently made from a very smooth fabric, smaller objects tend to slip away. On the other hand, participants appreciated the support during the gross motoric movements, which gave them a feeling of confidence. At the moment, the ironHand glove is not yet suitable for patients with more severe motor limitations.

\section{Limitations}

The sample of participants in the current study comprises mildly and moderately affected stroke patients, so the current findings cannot be generalized to more severely affected stroke patients. Other limitations of this study should also be noted. This study included a fairly small group size $(n=10)$. Therefore, outcomes should be interpreted with care. Additionally, the affected hand of the participants was not always the dominant hand. Task performance might differ when the affected hand is the dominant hand. On top of that, stroke patients often use their affected hand to support the non-affected hand, instead of using it to perform a reachand-grasp task as was done in the current study. Furthermore, the amount of time during which the participants used the system might not have been sufficient to completely familiarize with the system. In addition, even though the task was partly adapted to the person in terms of reaching range of motion and weight of the object, personal maximum performance was not defined precisely in the current study.

\section{Future direction}

The activated glove enhanced pinch strength without influencing the quality of movements. By contrast, wearing the glove in itself influenced the quality of movements. Although, the magnitude of those changes due to wearing a glove in itself is small, raising questions about the clinical relevance of those changes in quality of movement. Besides, it is possible that 
other factors, such as endurance, come into play when assessing the influence of a softrobotic glove on the performance of ADL. Given that the soft-robotic glove is designed to support grip strength, it is possible that the advantages of the glove become apparent in tasks involving prolonged usage of the hand. So, besides potential design adaptations (e.g., Velcro strap, donning/doffing, increase sensation, delay of grasping), future studies should focus on assessing the effect of the glove during tasks involving pinch strength and endurance aspects.

\section{Conclusion}

In conclusion, positive effects of the glove were seen when assessing pinch strength. On the contrary, negative effects on movement execution were found, in line with other studies investigating the direct effects of soft-robotic gloves. However, the current study revealed that this negative influence is due to wearing a glove in itself. Nevertheless, most of the alterations in joint excursion and other temporal parameters are so small, raising questions about the clinical relevance of such disturbance of spatial and temporal movement characteristics. Supported by the positive usability scores, it is probable that patients have made a trade-off between the gain in pinch strength and the slight reduction in movement quality. 



\title{
The effect of prolonged use of a wearable soft-robotic glove post stroke - a proof-of-principle
}

Chapter 7

\begin{abstract}
Many stroke patients encounter difficulties in the performance of activities of daily life due to limitations in functional use of the hand. Robotic technology has the potential to compensate for this loss by providing the support that is required to perform activities of daily living, especially when these devices are wearable comfortably for many hours at home. As a first step towards the implementation of assistive technology in the homes of stroke patients, usability along with the potential effect of prolonged use of a wearable soft-robotic glove during activities of daily life on functional task performance was assessed in this study. Therefore, five chronic stroke patients were asked to use a wearable soft-robotic glove for four weeks at home during preferred activities of daily life. Before and after the home use of the glove, functional task performance was assessed in a lab environment. After the use of the glove, system usability was assessed. The prolonged use of the glove resulted in an improved supported and unsupported functional performance during tasks related to activities of daily life, as measured with the Jebsen-Taylor Hand Function Test. Promising system usability results were found indicating a good probability for acceptance of the glove. The results from this study indicate the potential of the current glove to be used as assistive tool, which even showed a therapeutic effect. Yet, the glove should be tested in a larger sample for better interpretation and confirmation of these promising results.
\end{abstract}




\section{Introduction}

Limited independence of stroke patients associated with upper extremity impairments severely affects quality of life [12]. Only $10-15 \%$ of the stroke patients regain complete functional use in the activities of daily life (ADL) within six months after stroke [11]. The remaining proportion of the stroke patients often need support from formal and informal caregivers to overcome limitations in ADL.

Prolonged non-use of the hand in daily life resulting from the decreased hand function might limit the independence even more [14]. To promote use of the hand, intensive use of the affected hand by means of repetitive, task-specific therapy based on the essential principles of motor relearning is suggested [15-17]. New technologies have shown positive results on motor function after robot-assisted training of the upper extremity (UE) [10, 132, 152], but these devices could also be used as support during functional daily activities $[12,84,125]$. To allow prolonged support during ADL at home, a wearable three-fingered soft-robotic glove, the ironHand system, was developed.

Assistance of the hand during ADL might enable intensive use of the hand during functional activities, transforming assistive support into improved unsupported hand function after prolonged use in daily life. Several soft-robotic hand systems have been, and are being, developed for providing robot-assisted hand function in daily life [82, 153, 154]. However to our knowledge, only one previous study of our research group performed a first exploration of applicability of home use of a soft-robotic glove in two stroke patients so far, using an earlier and different, five-fingered, version of the current glove [139]. Use of former versions of the ironHand glove was feasible and perceived as usable both at home and in a lab environment $[108,109,138]$. Although some practical use issues were mentioned, the concept of the system was well received and appreciated by the users [109]. During the studies performed to assess the direct effect of the glove, no positive effects were found on functional task performance [108]. Nevertheless, Radder et al.[138] observed a learning curve in functional performance with glove but probably no plateau was reached yet. Prolonged use of such a glove might allow for more progression in functional task performance [129, 138].

As a next step towards use of a wearable assistive device in the home environment, the potential of an assistive glove while being used for a prolonged time unsupervised at home during ADL was assessed in the current study. Therefore, the direct effect, as well as its assistive and therapeutic potential on hand function were explored with five stroke patients. 


\section{Methods}

\section{Participants}

Chronic stroke participants were recruited from Eskilstuna Kommun Vård- och omsorgsförvaltningen, (Eskilstuna, Sweden) and Roessingh Center for Rehabilitation (Enschede, the Netherlands). Participants were included if they were above the age of 55 and living at home; suffered a first ischemic or hemorrhagic stroke resulting in a hemiparesis at least 6 months before inclusion; had limited motor function in their hand but with at least $10^{\circ}$ active flexion and extension of the fingers; and had no other impairments limiting hand and arm function or received currently hand therapy. This study was approved by local ethics committees and all participants provided written informed consent prior to the inclusion.

\section{Study design}

This longitudinal study consisted of two cross-sectional sessions (pre and post), with in between an intervention of four weeks in which participants were asked to use the glove during ADL. Participants were advised to use the glove for 180 minutes per week. They were free to choose during which activities, when and how long they wanted to use the glove. The direct effect of the glove was evaluated in the sessions pre- and post-intervention. Supported and unsupported functional performance were measured pre- and post-intervention to assess the effect of prolonged home use of the glove during $A D L$, i.e. the assistive and therapeutic effect. At the end of the post-intervention session, the system usability was assessed by means of the system usability scale questionnaire [143].

\section{Soft-robotic glove}

The ironHand system is a wearable soft-robotic glove. The system consists of a three-fingered glove and a control unit that contains the embedded software to control the amount of force needed to support grip strength, and the batteries. Grip strength of the hand is supported by the glove via the thumb, middle and ring finger. The amount of support is regulated by a tendon-driven mechanism, receiving input from pressure sensors (Interlink Electronics) located at the fingertips of the fingers. The force added to the grip of the user is provided by the actuators, and is in proportion to the grip force applied by the user. The level of sensitivity was set on a level matching the participants' needs and experienced comfort.

\section{Evaluation}

The evaluation sessions pre- and post-intervention took place in a lab environment in the Netherlands or Sweden. To assess motor status and degree of synergy development preintervention, the upper extremity part of the Fugl-Meyer assessment was used [88]. A maximum of 66 points can be assigned. 
A hydraulic hand dynamometer (Jamar hydraulic hand dynamometer, Patterson Medical Ltd., Warrenville, IL, USA) was used to assess the maximal grip strength both pre- and postintervention. A standardized position was used as described by the American Society of Hand Therapists [115]. The participants were asked to squeeze three times maximally for five seconds with their affected hand. Between the attempts were at least 60 seconds of rest. The best of the three attempts was included in the analysis. This test was executed both supported and unsupported, and the maximal support provided by the glove was set at $20 \mathrm{~N}$ for every subject.

To assess functional performance of the supported and unsupported affected hand during tasks related to ADL, the Jebsen-Taylor Hand Function Test (JTHFT) was performed pre- and post-intervention. The JTHFT consists of seven different unilateral hand skills tasks: 1 ) writing one sentence of 24 letters; 2) turning over cards; 3) picking up small, common objects and move these to a box; 4) simulated feeding; 5) stacking checkers; 6) picking up large empty cans, and; 7) moving weighted cans $[155,156]$. The writing-task was omitted from the analysis afterwards, because of the variation in hand dominance related to the affected hand between participants. Time needed to complete the task in seconds was recorded with a stopwatch, and the time was summated for the total score.

At the end of the post evaluation session, system usability was assessed with the System Usability Scale (SUS) [143]. It uses a 5-point Likert scale for 10 questions about system usability. The answers can range from 'strongly disagree' to 'strongly agree'. The total score of the questions was multiplied by 2.5, so that the maximum score is 100 [143], with higher scores indicating better usability of the system. A score below 50 predicts usability difficulties in the field, a SUS score between 50-70 indicates fair usability and scores above 70 indicates a good probability of acceptance or even excellent usability for scores above 85.5 [89, 91]. In addition, participants were asked to report any adverse events to assess the practical applicability of the system.

\section{Data analysis}

Descriptive statistics of all outcome measures were reported as median with interquartile range (IQR, 25th - 75th percentile). In addition, SUS scores were presented per individual. To assess the direct influence of the glove, performance with and without glove pre-intervention were compared. Supported functional performance with glove post-intervention was compared to performance with glove pre-intervention to assess the assistive effect of using the glove for four weeks at home. Additionally, unsupported functional performance without glove was compared pre- and post-intervention to assess the therapeutic effect. Due to the small sample size, outcomes were non-parametrically tested for statistical significance with the Wilcoxon 
signed ranks test. Statistical analysis was performed with IBM SPSS Statistics 19, and effects were considered statistically significant for $p<0.05$.

\section{Results}

\section{Participants}

Five chronic stroke patients participated in this study. Patient characteristics and FM scores are summarized in Table 7.1. Of one of the participants no FM was registered, two participants had moderate hemiparesis $(26 \leq \mathrm{FM} \geq 50$ ) and two participants had mild hemiparesis ( $F M>50)$.

Table 7.1 Patient Characteristics.

\begin{tabular}{lll}
\hline Variable & N & Outcome \\
\hline Age & 5 & 65 (IQR: 57-73) \\
Gender & 5 & 2 male/ 3 female \\
Affected body side & 5 & 2 right/ 3 left \\
Dominant body side & 5 & 3 right/ 2 left \\
FM upper extremity score & 4 & 45 (IQR: 36-54) \\
\hline
\end{tabular}

\section{(Functional) performance}

Median scores and IQR of the maximal pinch strength test are presented in Table 7.2. Maximal grip strength did not significantly change as direct effect of the glove $\left(p_{\text {pre }}=1.00\right.$ and $p_{\text {post }}=$ $0.18)$, or after prolonged use of the glove at home $\left(p_{\text {assistive }}=0.72\right.$ and $\left.p_{\text {therapeutic }}=0.41\right)$.

Table 7.2 Outcome measures.

\begin{tabular}{lrcc}
\hline Condition & Time & \multicolumn{2}{c}{ Variable } \\
\hline \multirow{2}{*}{ With glove } & & Maximum Grip Strength (kg) & JTHFT (seconds) \\
& PRE & $15.90(14.75-21.00)$ & $206.54(71.96-369.10)$ \\
\multirow{2}{*}{ Without glove } & POST & $16.00(13.00-21.50)$ & $118.62(57.92-315.80)$ \\
& PRE & $15.90(14.00-22.50)$ & $175.99(56.56-404.44)$ \\
& POST & $16.50(15.50-22.00)$ & $134.43(68.68-346.13)$ \\
\hline
\end{tabular}

There was no significant direct effect of the glove found on the time needed to perform the JTHFT ( $p_{\text {pre }}=0.96$ and $p_{\text {post }}=0.89$ ). Post-intervention, a significant decrease in time needed to perform the JTHFT (improved performance) was found both in supported ( $p_{\text {assistive }}=0.04$ ) and unsupported condition $\left(p_{\text {therapeutic }}=0.04\right)$. 
Time needed to perform the different subtasks of the JTHFT are presented in Figure 7.1. Without glove, participants needed less time to turn cards $(p=0.04)$, and to pick up empty ( $p$ $=0.04)$ and weighted cans $(p=0.04)$ after four weeks of glove use. With glove, time needed to turn cards $(p=0.04)$ and to pick up empty cans $(p=0.04)$ also decreased significantly postintervention as compared to pre-intervention.

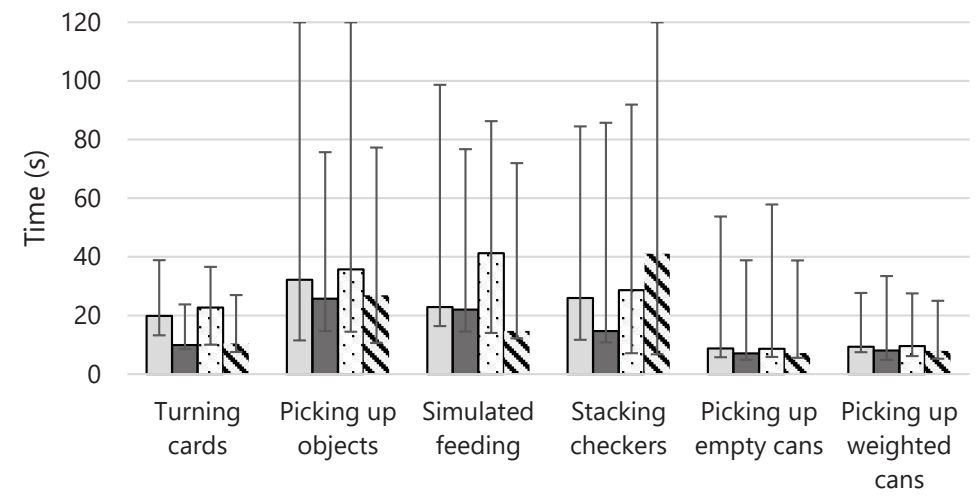

$\square$ Pre With glove $\square$ Post With glove $\square$ Pre Without glove $\mathbf{S}$ Post without glove

Figure 7.1 Time, presented as median value and IQR, needed to perform subtasks of the JTHT before intervention with glove and without glove, and after intervention with and without glove. * indicates $\mathrm{p}<0.05$.

\section{System usability}

Usability of the glove was rated as good (1 participant) to excellent (4 participants), with SUS scores ranging from 75-100 (median score of 87.5). Individual scores are presented in Figure 7.2. No adverse events were reported by the participants during the course of the study.

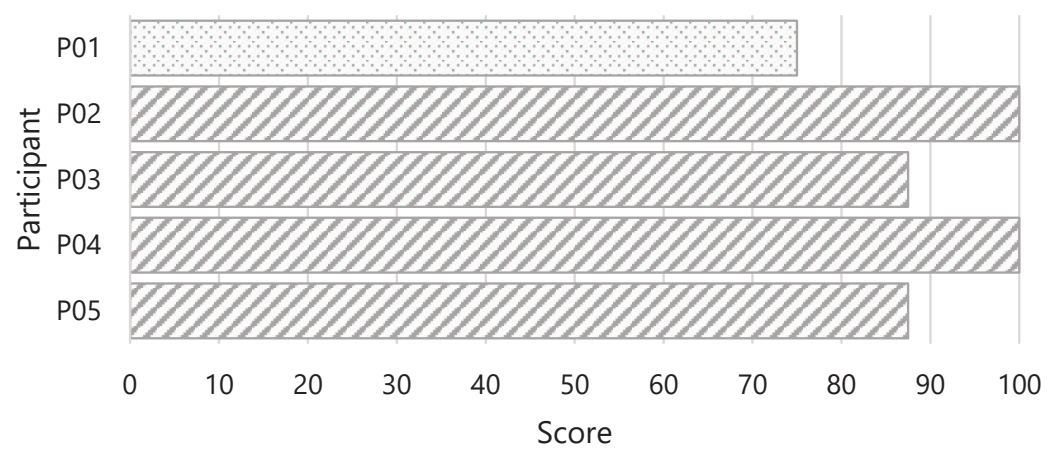

Figure 7.2 Individual SUS Scores. Bars filled with dots indicate scores $>70$, Upward diagonal filled bars indicate scores $>85$. 


\section{Discussion}

The current study is one of the first studies in which a fully wearable assistive device for the hand was used for four weeks unsupervised during ADL in the patient's home. As a first step towards the implementation of assistive technology in the homes of stroke patients, usability along with potential clinical changes of the prolonged use of a wearable soft-robotic glove during $A D L$ on functional task performance were explored in this study among five stroke patients. Our findings showed good to excellent perceived usability of the glove among the participants when using it at home, suggesting a high chance of adoption in daily life [91]. In addition, both supported and unsupported hand function during tasks related to ADL improved after four weeks of glove use as assistive device at home, implying an assistive and therapeutic effect.

Remarkably, no significant effect was found on grip strength, even though the glove was developed to support grip strength. However, this is likely due to a suboptimal positioning of the pressure sensors of the glove to perform the maximum grip strength test as applied in this study. A custom designed pressure sensor, such as a cylinder, should be considered in future research $[84,154]$.

Additionally, there was no significant direct effect of the glove on the time needed to perform the JTHFT, both pre- and post-intervention. The JTHFT was performed even slightly slower with glove than without pre-intervention. Similar findings were observed by Polygerinos et al.[125], in which a healthy subject performed the JTHFT tasks slower with a glove controlled by EMG developed for assistive and therapeutic purposes [84, 125]. Compared to normative data, the healthy subject performed several JTHFT tasks slower with glove in a direct effect study [125]. As suggested by Radder et al.[138], more time to get used to the system might be needed to find effects of the glove. This suggestion is confirmed by the results of this study with regard to the assistive effect of the glove, since supported JTHFT was performed significantly faster after four weeks of home use of the glove. Although the glove was reported as easy and intuitive to use [138], these results imply that participants need time to familiarize with the system and use it on regular basis in order to enhance their functional performance. These results might have implications for the implementation of the glove in practice.

Especially during the performance of gross motoric subtasks, such as picking up cans, participants improved after the intervention period. This result is in line with previous findings that the glove would be more useful to support gross motor activities [139]. However, also improvements in supported and unsupported execution of fine motoric subtask were observed in the present study, involving turning cards and to a lesser extent picking up small objects. The subtask simulated feeding was also performed faster, with a trend towards statistical 
significance. Although no results have been published so far about the longitudinal evaluation of an assistive glove to assist hand function at home post-stroke, the therapeutic effect is seen as well in studies examining the therapeutic effect of robotic technology used for upper extremity rehabilitation $[44,45,157,158]$. In these studies, a therapeutic effect on upper extremity functioning was shown after six weeks (or more) home training with a robotic device $[44,45,157,158]$. However, inconclusive findings were reported about the existence of this therapeutic effect two months post-intervention $[45,157]$. In contrast to technology designed for home-based rehabilitation, the current glove has the potential to continue the assistance, and possible training, in everyday life without taking additional time for therapy.

The present findings need cautious interpretation due to the small sample size. Usability of the soft-robotic glove at home was rated as good to excellent, implying a high chance of adoption in daily life. In addition, no adverse events were reported by the participants, showing the practical applicability of home use of the wearable soft-robotic glove. Before implementing the glove at home, design improvements need to be taken into account, such as the ability to use the glove with water and easier donning/doffing [138]. Besides, usage time of the glove was not measured in the current study, while 180 minutes per week for six weeks was advised to achieve clinically relevant functional improvements [159].

Despite the mentioned recommendations for future development and testing, our findings suggest a potential therapeutic effect, as a result of using the glove during ADL. Since usually not only the hand but also the arm is affected after stroke, adding the glove to other modules supporting the arm and/or wrist, depending on the physical limitation of the user, might benefit stroke patients even more. The eNHANCE project currently explores the possibility to connect the glove with an arm and/or wrist support to provide assistance to a larger part of the stroke population during ADL in the future. Furthermore, additional research is needed with a larger sample size to underpin the results found in this study and to assess the effects of using the glove for a longer period of time during ADL.

\section{Conclusion}

Home-use of the wearable soft-robotic glove during ADL showed promising effects on supported and unsupported performance of ADL-related tasks in stroke patients, indicating a possible assistive but also therapeutic effect of the assistive glove. Results from the current study suggest that it is feasible to use a soft-wearable glove unsupervised at home to assist ADL with a high probability of adoption. In future studies, a next version of the glove should be tested in a larger sample to underpin the found results. 



\title{
Detection of the intention to grasp during reach movements
}

Chapter 8

\begin{abstract}
Soft-robotic gloves have been developed to enhance grip to support stroke patients during daily life tasks. Studies showed that users perform tasks faster without the glove as compared to with the glove. It was investigated whether it is possible to detect grasp intention earlier than using pressure sensors to enhance the performance of the glove. This was studied by distinguishing reach-to-grasp movements from reach movements without the intention to grasp, using minimal inertial sensing and machine learning. Both single-user and multi-user support vector machine classifiers were investigated. Data were gathered during an experiment with healthy subjects, in which they were asked to perform grasp and reach movements. Experimental results show a mean accuracy of $98.2 \%$ for single-user and of $91.4 \%$ for multiuser classification, both using only two sensors: one on the hand and one on the middle finger. Furthermore, it was found that using only $40 \%$ of the trial length, an accuracy of $85.3 \%$ was achieved, which would allow for an earlier prediction of grasp during the reach movement by $1200 \mathrm{~ms}$. Based on these promising results, further research will be done to investigate the possibility to use classification of the movements in stroke patients.
\end{abstract}




\section{Introduction}

Motor impairment of the upper extremities due to stroke results in limited performance of daily life tasks. Worldwide, there are 62 million stroke patients [160], of which 77\% suffer from upper limb motor deficits [7]. From this group, at least 60 percent failed to incorporate the affected hand in their daily life [11]. One of the major limiting factors is that they experience difficulties with grasping objects, due to muscle weaknesses, atypical muscle synergies, and spasticity [161]. Task-oriented therapy shows great benefits for regaining function in the affected hand, as compared to other physical therapies [159, 162]. To support the patients' grasp at home, several soft-robotic gloves have been developed to enhance grip, such as the $\mathrm{SEM}^{\mathrm{TM}}$ Glove (Soft Extra Muscle Glove) from Bioservo Technologies AB. Using pressure sensors, it detects when the user grasps an object, which subsequently activates the grasp support [163].

A study on the feasibility of the ironHand (a soft-robotic glove based on the concept of the $\mathrm{SEM}^{\mathrm{TM}}$ Glove) has shown that a mean System Usability Score of $70.1 \%$ was achieved [138], which indicates a good probability for acceptance by the users. However, the study also showed that the subjects performed tasks significantly faster without the glove as compared to with the glove [109], since the glove detects the intention to grasp using interface pressure sensors, which allows grasp detection not earlier than the moment at which an object is actually touched. These findings are in line with the study of Polygerinos et al.[84] with an EMG controlled soft-robotic glove. To further enhance the performance of daily life tasks of the users while using a soft-robotic glove, it could be of interest to detect the intention to grasp an object as early as possible, e.g. using inertial sensing. In case the intention to grasp is detected earlier than using the pressure sensors, the support could be activated earlier, which might result in improved performance.

Several studies have been done to detect grasp intention in healthy subjects $[126-128,164$, 165]. These studies investigated whether it was possible to detect and predict the final hand posture as early as possible. Using position data from bend sensors and pressure sensors, De Souza et al.[126] achieved a recognition rate of $87 \%$ with multiple subjects. Heumer et al.[164] showed that a highly reliable recognition of grasp types can be achieved using bend sensors, if the user of the data glove trains the classifier (single-user classification); a reasonably good recognition was achieved for users who were not among those who trained the classifier (multi-user classification). Ekvall and Kragic[127] showed that the positions of the fingertips are very important to predict the final hand posture, as well as the roll angle and roll angle velocity of the hand. Furthermore, they showed that their method recognized the final hand postures with a $95 \%$ accuracy at $60 \%$ of grasp completion (the moment the subject touches the object), for a single-user model. For a multi-user model, the model performed optimally 
at $80 \%$ of grasp completion, with an accuracy of $65 \%$. Naish et al.[128] used electromagnetic sensors in combination with electromyography, to study the difference between grasp-to-eat and grasp-to-place movements. They showed that there were significant differences between the movements in timing of peak acceleration of thumb, index finger, and wrist.

These studies show promising results regarding grasp recognition using classification methods. However, to detect grasp intention before it can be detected by interface pressure sensors, the algorithm should be able to distinguish a reach-to-grasp movement from a reach movement. None of the above studies investigated whether they could distinguish a reachto-grasp movement from a reach movement without grasp (e.g. pointing at something or waving). All studies only included reach-to-grasp movements with different kinds of grasps in their experiments. Therefore, an experiment should be done containing both movements. In addition, all studies used multiple sensors to detect the grasp intention. However, it is of interest to investigate the minimal number of sensors needed to accurately distinguish between the two movements: the fewer sensors are needed, the more robust the system will be for application and the easier and cheaper it is to implement the sensors in soft-robotic gloves. It was hypothesized that a minimal number of sensors could be achieved by detecting reach-to-grasp movements using hand opening and closing or flexion and extension of the wrist. This was measured using the relative angular velocity of the fingers and forearm with respect to the hand respectively.

Hence, the goal of this study was to investigate the best sensor combination for the classification of reach-to-grasp and reach movements without the intention to grasp, based on tracking of hand and finger movement using inertial sensing. Subsequently, it was investigated whether it was possible to predict the intention to grasp during reach movements. Both single-user and multi-user classification were studied using support vector machine (SVM) classifiers.

\section{Methods}

\section{Subjects}

Sixteen healthy subjects were recruited for the experiment ( 6 male, 10 female; age: $24.78 \pm 7.3$ years; all dominant right-handed). Subjects met the following inclusion criteria: (1) the subject should be at least 18 years old; (2) be healthy with full arm function intact; and (3) be dominant right-handed. Exclusion criteria were: (1) motor impairment; and (2) wounds or other limiting factors on the hand for applying the sensors or the glove or while performing the tasks. All subjects provided informed consent prior to the start of the experiment. Ethical clearance was obtained from a local ethics committee. 


\section{Instrumentation}

The angular velocities of the hand segments were measured using an inertial measurement system [166], with inertial measurement units (IMUs) placed on phalanges of the fingers, thumb, dorsal side of the hand and distal end of the arm. The sensors' placings chosen to be analyzed to detect flexion and extension of the fingers were the distal phalanges of the thumb, index finger, and middle finger, and the dorsal side of the hand. To detect flexion and extension of the wrist, an additional sensor was placed on the distal end of the forearm on the dorsal side, next to the ulnar styloid process.

Next to the IMUs, the SEM ${ }^{\mathrm{TM}}$ Glove from Bioservo Technologies AB[163] was used. The SEM ${ }^{\mathrm{TM}}$ Glove records the time at which the pressure sensors detect that the user grasps objects.

\section{Set-up}

The table was prepared by taping the directions of five horizontal locations $\left(0^{\circ}, 45^{\circ}, 90^{\circ}, 135^{\circ}\right.$, and $180^{\circ}$ ). The distances of the locations were determined using the reaching range of motion of the subject. The initial hand position was located in the middle of the semicircle, at the edge of the table, with the elbow flexed to $90^{\circ}$ and held close to the body. See Figure 8.1 for a schematic of the set-up.

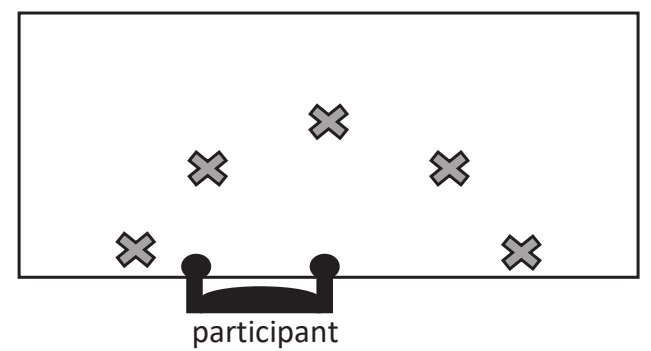

Figure 8.1 Task locations for the experiment. The subject is seated with his right hand in the middle of the circle.

\section{Protocol}

The experiment was started with performing exercises for the sensor-to-segment calibration (as described in the next section). Then, the subjects were verbally instructed to perform four different tasks towards one of the five locations: (1) grasp a bottle of water (2) grasp a tennis ball; (3) reach to hold the hand above location with the palmar side of the hand facing downward towards the table; and (4) reach while supinating the arm to hold the hand above location with the dorsal side of the hand facing downward towards the table. The grasp-related tasks represent the two most common grasps in stroke patients: cylindrical and spherical grasps [26]; the reach-related tasks closely resemble the grasp-related tasks, 
with respect to the direction of movement and the rotation of the lower arm. The tasks were performed six times for each location. Besides, all tasks were performed for two different initial hand postures, i.e. (1) opened hand, resting on the table with the palmar side of the hand facing down; and (2) a fist, resting on the table with the medial side of the hand facing down. In total, the subject performed 120 grasp and 120 reach movements. The order of the tasks was randomized, as well as the order of the initial hand posture and the locations.

\section{Sensor-to-segment calibration}

Each experiment started with a sensor-to-segment calibration as described by Luinge et al.[167] in order to estimate the anatomical rotation axes. For the calibration, the subject was asked to perform the following tasks: (1) to stand upright with the elbow of the right arm flexed in $90^{\circ}$ and the dorsal side of the hand facing upwards, (2) to flex and extend the fingers, (3) abduct and adduct the fingers and thumb, (4) flex and extend the thumb, (5) flex and extend the wrist, (6) adduct and abduct the wrist, and (7) to flex and extend the elbow.

The coordinate systems of all segments are defined according to the coordinate system of the whole body when in anatomical position, i.e. the $x$-axis is defined to be the anteriorposterior axis pointing forward, the $y$-axis is the medial-lateral axis pointing to the right, and the $z$-axis is the vertical axis pointing down. The $x$-axis represents the movements abduction and adduction; the $y$-axis flexion and extension; and the $z$-axis supination and pronation. The direction of the $y$-axis was determined using the direction of the angular velocity during flexion (second task for the fingers, fourth task for the thumb, fifth task for the dorsal side of the hand, and seventh task for the lower arm); the direction of the $x$-axis was determined by measuring the direction of gravity while standing upright with the elbow flexed to $90^{\circ}$ (first task); the direction of the $z$-axis was determined using the cross-product of the direction of the $x$ - and $y$-axis. To make the system orthogonal, the direction of the $x$-axis was recomputed using the cross-product of the $y$-axis and the $z$-axis. Using the rotation matrices containing the three unit vectors of the segments, the data from the gyroscopes measured in the IMU coordinate systems were rotated to the coordinate systems of the body segments [167].

\section{Data analysis}

Pre-processing. The data from the gyroscopes and the SEM ${ }^{\mathrm{TM}}$ Glove were pre-processed by removing the bias. The bias of the sensors was removed by manually selecting a baseline at the beginning of the recording, in which there is no movement of the hand and no force on the SEM ${ }^{\mathrm{TM}}$ Glove pressure sensors. The data from the IMUs were then filtered with a 4 th order, zero-lag, low pass Butterworth filter with a cut-off frequency of $6 \mathrm{~Hz}[168,169]$.

Classification. Two discrete classes were defined for the classification: Grasp and Reach. Since 
an SVM classifier is able to find patterns in high dimensional, nonlinearly separable data and since it is very accurate for classification of exactly two classes, the SVM classifier was used for classification of the data $[170,171]$. Classification was done for both single-user and multiuser scenarios. In case of a single-user classifier, the classifier was trained and tested by the same subject. In case of a multi-user classifier, the classifier was trained by all subjects except one and tested by the subject excluded from the training set. The SVM classifiers were trained using 10-fold cross-validation. Six kernels were investigated: the Linear kernel, Quadratic kernel, Cubic kernel, Fine Gaussian kernel, Medium Gaussian kernel and the Coarse Gaussian kernel. The accuracy of all kernels was investigated for five subjects for both single-user and multi-user classification, after which the best kernel was selected for further analysis with all subjects.

Database. The data from the gyroscopes were cut into small pieces, which are called 'trials'. One trial contains the data from the moment the subject starts performing one of the tasks, until the moment that the subject either grasps the object, or reaches the location for the reach-related tasks. The beginning and end of the trials were found using a threshold detector algorithm for the angular velocity, with a threshold of $\pm 0.1 \mathrm{rad} / \mathrm{s}$. In case of the grasp-related tasks, the end of the trial was considered the moment the SEM ${ }^{\mathrm{TM}}$ Glove detected the grasp. The data were cut into 240 trials per subject. After selecting the trials for data analysis, the resulting database was subdivided into a training set and a test set. The minimum number of trials needed to train the classifier accurately was 70, as examined using a learning curve [172] from data obtained during a trial prior to this experiment. Two different types of databases were created: for single-user and for multi-user classification.

Single-user database. From both the grasp and reach trials, 25\% was randomly selected for the test set. Hence, the test set consisted of 60 trials (30 grasp, 30 reach) and the training set consisted of 180 trials (90 grasp, 90 reach).

Multi-user database. The database for multi-user classification consisted of the trials of one subject in the test set, and the trials of the remaining subjects $(n-1)$ in the training set. In total $n$ databases were created; one database for each test subject.

Feature extraction. To easily detect flexion and extension of the fingers and wrist, all segment movements were expressed with respect to the dorsal side of the hand. This was done using the norm of the angular velocity vectors and using the relative angular velocity of the segments. The norm of the angular velocity vector defines the movement of segment $a$ with respect to the dorsal side of the hand using the norm ${ }_{a}$ of the difference in angular velocity between the $x-, y^{-}$, and $z$-axis of the segments and hand. The relative angular velocity of a segment with 
respect to the dorsal side of the hand $\left(\omega_{\mathrm{a}}\right)$ was expressed using sensor-to-segment calibration. The angular velocity from the dorsal side of the hand was subtracted from the angular velocity of other segments, after performing the sensor-to-segment calibration for both segments. The $x$-axis represents $\mathrm{ab}$ - and adduction of the fingers and wrist, the $y$-axis flexion and extension, and the $z$-axis pronation and supination. For all trials, the mean and standard deviation (SD) were determined for the norm of the angular velocity vectors and for the relative angular velocities. These features were used as input for the SVM classifier. In total, 8 features were extracted from the trials based on the norm of the angular velocity vectors of the segments 4 segments $x 2$ (mean and SD)); and 24 features were extracted based on the relative angular velocity of the segments (4 segments $x 3$ directions $(x, y$, and $z) \times 2$ (mean and SD)).

Feature selection. To find the best sensor combination for the classification of reach-tograsp and reach movements without the intention to grasp (first goal of this study), the best predicting features were found by training SVM classifiers for 14 different combinations of the features, see Table 8.1. The selection of the combinations was based on combining only two sensors: the sensor on the dorsal side of the hand with one of the other segments; on the output measures: both the norm of the angular velocity vector and the relative angular velocity were investigated; and on the directions of movement for the relative angular velocity: all directions were investigated $\left(x^{-}, y^{-}\right.$, and $z$-axis) as well as the direction representing flexion and extension ( $y$-axis). Apart from the $y$-axis, the $x$-axis and $z$-axis were studied for the thumb as well, since the thumb can easily move over these axes due to the saddle joint. The training of all 14 combinations was done for five (test) subjects for both single-user and multi-user classification, after which the two best combinations were selected for further analysis with all subjects. The selection of the best features and SVM classifier was based on the accuracy of the classifier, visual inspection of the scatter plots of the features, the ROC-curve of the classifier (showing sensitivity relative to specificity), and the training and prediction speed of the classifier.

Grasp intention detection. In order to investigate whether it was possible to predict the intention to grasp during reach movements (second goal of this study), the influence of selecting only a certain part (\%) of the signal for both training and testing of the classifier on the accuracy of the classifier was analyzed. This was investigated using the data sets for singleuser classification. The part of the signal selected for training and testing (e.g., a classifier trained using $30 \%$ of the trial length was tested on a test set with also $30 \%$ of the trial length) is from the beginning of the trial until a certain percentage of the original trial length. 
Table 8.1 Combinations of features used to train SVM classifiers. In total, the SVM classifiers were trained for 14 different combinations of features.

\begin{tabular}{|c|c|c|c|}
\hline$\#$ & Features & Output measure & Segment $a$ \\
\hline 1 & Mean + SD & norm $_{a}$ & Middle finger \\
\hline 2 & Mean + SD & norm $_{a}$ & Index finger \\
\hline 3 & Mean + SD & norm $_{a}$ & Forearm \\
\hline 4 & Mean + SD & norm $_{a}$ & Thumb \\
\hline 5 & Mean + SD & $\omega_{a}(x-y-$, and $z$-axis $)$ & Middle finger \\
\hline 6 & Mean + SD & $\omega_{a}(x-y-$, and $z$-axis $)$ & Index finger \\
\hline 7 & Mean + SD & $\omega_{a}(x-, y-$, and $z-a x i s)$ & Forearm \\
\hline 8 & Mean + SD & $\omega_{a}(x-y-$, and $z$-axis $)$ & Thumb \\
\hline 9 & Mean + SD & $\omega_{a}(y$-axis) & Middle finger \\
\hline 10 & Mean + SD & $\omega_{a}(y$-axis $)$ & Index finger \\
\hline 11 & Mean + SD & $\omega_{a}(y$-axis $)$ & Forearm \\
\hline 12 & Mean + SD & $\omega_{a}(y$-axis $)$ & Thumb \\
\hline 13 & Mean + SD & $\omega_{a}(x-a x i s)$ & Thumb \\
\hline 14 & Mean + SD & $\omega_{a}$ (z-axis) & Thumb \\
\hline
\end{tabular}

\section{Results}

\section{Subjects}

Due to hardware malfunction, five subjects were excluded from data analysis. Therefore, the data of 11 subjects were analyzed ( 4 male, 7 female; age: $25.7 \pm 8.6$ years; all dominant righthanded).

\section{Kernel and feature selection}

Based on visual inspection of the scatter plots of the features (for an example, see Figure 8.2) and on the accuracies of the SVM classifiers after cross-validation (Figure 8.3), the two best feature combinations were selected: the mean and SD of the relative angular velocity about the anatomical $x-, y-$, and $z$-axis middle finger and index finger (combination 5 and 6 , respectively, as listed in Table 8.1 ). 


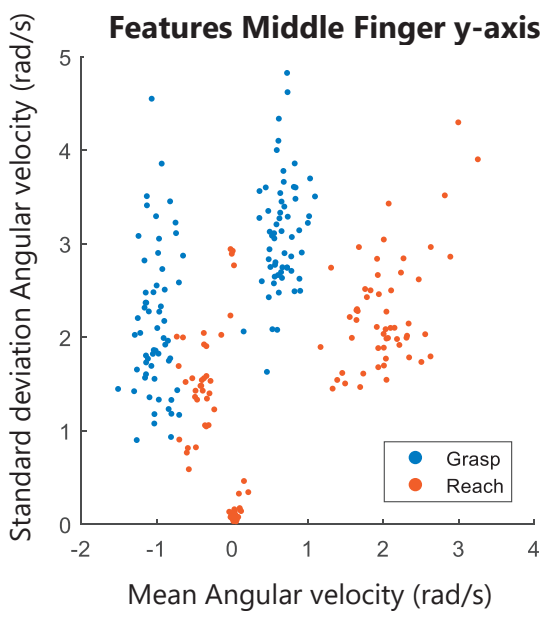

Figure 8.2 Scatter plot of the features mean and SD of the relative angular velocity about the anatomical $y$-axis of the middle finger. The $y$-axis represents flexion and extension.

The $x$-axis represents $a b$ - and adduction, the $y$-axis flexion and extension, and the z-axis pronation and supination. The kernel achieving the highest accuracy for single-user classification was the Cubic kernel, for multi-user classification the Fine Gaussian kernel (Table 8.2). The ROC-curve, training and prediction speed were analyzed as well, but did not show considerable differences.
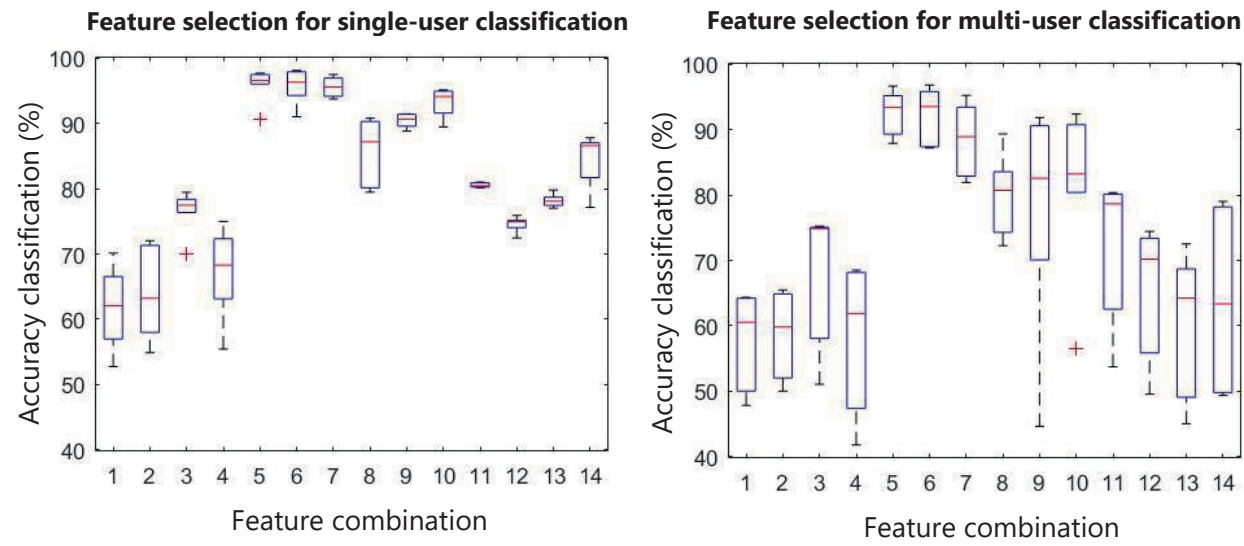

Figure 8.3 Boxplots showing the accuracies of single-user (left) and multi-user (right) SVM classifiers for all feature combinations. The SVM classifiers were trained using six different kernels for each feature combination. The numbers representing feature combinations correspond with he numbers in Table 8.1. 
Table 8.2 Mean kernel performance for single-user and multi-user classification of the top two feature combinations.

\begin{tabular}{lll}
\hline Kernel & $\begin{array}{l}\text { Single-user } \\
\text { Mean } \pm \text { SD }(\%)\end{array}$ & $\begin{array}{l}\text { Multi-user } \\
\text { Mean } \pm \text { SD (\%) }\end{array}$ \\
\hline Linear & $95.2 \pm 1.0$ & $85.7 \pm 3.3$ \\
Quadratic & $97.0 \pm 0.5$ & $90.4 \pm 2.7$ \\
Cubic & $97.0 \pm 0.3$ & $94.4 \pm 0.9$ \\
Fine Gaussian & $94.8 \pm 1.1$ & $96.2 \pm 0.9$ \\
Medium Gaussian & $96.7 \pm 0.3$ & $93.8 \pm 2.9$ \\
Coarse Gaussian & $91.7 \pm 1.7$ & $86.5 \pm 3.3$ \\
\hline
\end{tabular}

Note: The best feature combinations are combination 5 and 6 (see Figure 8.3).

\section{Classification single-user}

Table 8.3 shows the accuracy of classifier predictions for the two best single-user classifiers. The highest mean accuracy of $98.2 \%$ was achieved using a Cubic SVM classifier, with the features mean and SD of the relative angular velocity of the middle finger about the anatomical $x-, y-$, and $z$-axis (combination 5). The mean training speed is $0.83 \mathrm{~s}$ and the mean prediction speed is 7400 observations/s. The best classifier is closely followed by the classifier with the features regarding the relative angular velocity of the index finger, with an accuracy of $97.6 \%$.

\section{Classification multi-user}

Table 8.3 also shows the accuracy of classifier predictions for the two best multi-user classifiers. The highest mean accuracy of $91.4 \%$ was achieved using a Fine Gaussian SVM classifier, with the features mean and SD of the relative angular velocity of the middle finger about the anatomical $\mathrm{x}-, \mathrm{y}-$, and $\mathrm{z}$-axis. The mean training speed is $2.2 \mathrm{~s}$ and the mean prediction speed is 34,000 observations/ $\mathrm{s}$. The next best feature for multi-user classifier is the relative angular velocity of the index finger about the anatomical $x_{-}, y_{-}$, and $z$-axis, with an accuracy of $91.2 \%$.

Table 8.3 Accuracy of classifier predictions for single-user and multi-user classifiers.

\begin{tabular}{llll}
\hline \multicolumn{2}{l}{ Single-user classification - Cubic kernel } & \multicolumn{2}{l}{ Multi-user classification - Fine Gaussian kernel } \\
& Mean \pm SD $(\%)$ & Features & Mean \pm SD (\%) \\
\hline Middle finger & $98.2 \pm 0.02$ & Middle finger & $91.4 \pm 0.06$ \\
Index finger & $97.6 \pm 0.03$ & Index finger & $91.2 \pm 0.07$ \\
\hline
\end{tabular}

Note: The single-user classifiers were trained using a training set of 180 trials and a test of 60 trials. The multiuser classifiers were trained using a training set of 2400 trials from 10 subjects and a test set of 240 trials. 


\section{Grasp intention detection}

Table 8.4 shows the results of training and testing classifiers with only a part of the signal. Using only $40 \%$ trial length, a mean accuracy of $85.3 \%$ was achieved. To achieve an accuracy of at least $90 \%$, grasp intention was detected at $70 \%$ trial length. The average duration of a reach-to-grasp movement is $2.05 \mathrm{~s}$. Hence, detecting the grasp intention at $70 \%$ trial length makes the detection of grasp intention approximately $600 \mathrm{~ms}$ earlier as compared to the SEM $^{\text {TM }}$ Glove; and at 40\%, 1200 ms earlier.

Table 8.4 Mean kernel performance for single-user and multi-user classification of the top two feature combinations.

\begin{tabular}{ll}
\hline Trial length & Mean \\
\hline $10 \%$ & $69.5 \%$ \\
$20 \%$ & $78.6 \%$ \\
$30 \%$ & $81.3 \%$ \\
$40 \%$ & $85.3 \%$ \\
$50 \%$ & $84.5 \%$ \\
$60 \%$ & $87.1 \%$ \\
$70 \%$ & $90.6 \%$ \\
$80 \%$ & $95.1 \%$ \\
$90 \%$ & $98.0 \%$ \\
\hline
\end{tabular}

Note: The SVM classifier was trained using a training set of 180 trials, a Cubic kernel, and 10-fold cross-validation. The features used for training were the mean and SD of the relative angular velocity of all anatomical axes of the middle finger. The trials used for training contained only a part (\%) of the original trial. The trained classifiers were tested on a test set containing the corresponding part of the trials.

\section{Discussion}

The results of the experiment clearly show that it is possible to distinguish reach-to-grasp from reach movements without the intention to grasp using machine learning. High accuracies were achieved for both single-user (98.2\%) and multi-user classification (91.4\%) using only two sensors: one on the dorsal side of the hand and one on the distal phalange of the middle finger. Compared to related work, the results are very promising: the single-user classifier achieved a similar accuracy to the one found by Heumer et al.[164] and the multi-user classifiers achieved a higher accuracy than found by De Souza et al.[126] and Heumer et al.[164]. The performance of the multi-user classifier could be enhanced by scaling the features to for example the maximum velocity used by the user. Since every user uses a different velocity for opening the hand, it could be easier to distinguish the two classes Grasp and Reach using feature scaling in case of multi-user classification. 
The results for grasp intention detection, as investigated using only a part of the trials, are very promising for the development of a real-time grasp intention algorithm. The detection is 600 to $1200 \mathrm{~ms}$ earlier with an accuracy of $90.6 \%$ and $85.3 \%$, respectively. Hence, depending on the accuracy that is preferred to achieve, the IMU sensors can distinguish the movements much earlier than the pressure sensors detect the grasp. This is essential in order to improve grasp-supporting devices, such as the $\mathrm{SEM}^{\mathrm{TM}}$ Glove, since at this point in time, it takes longer to grasp with the supporting glove as compared to without the glove. A trade-off must be made between the preferred accuracy, and the preferred time between the prediction and the actual grasping of the object.

When developing the real-time grasp intention algorithm, the method used to filter the signal should be changed compared to the method used in this study. Since the data were filtered off-line, it was possible to filter the data zero-lag. However, this is not possible in real-time detection. Therefore, a latency will be introduced by the filter. How big the latency will be, depends on the filter and window used. As an alternative for classification using machine learning, it could be of interest to look at a threshold detector in combination with a matched filter for real time detection. Using a matched filter, it is possible to analyze the signal over time, which could hold very useful information. This information is not used in the machine learning methods that have been evaluated in the current study.

During training of the SVM classifiers, it was found that the accuracy differs considerably when different kernels are used. Since it is preferred to define one SVM classifier to be trained, an optimal combination of kernel and features was found which resulted on average in the highest accuracy. Hence, it could be that there are better performing SVM classifiers for single users than the average best classifier. Therefore, when using single-user machine learning to detect grasp intention, it could be considered to find the best kernel for each user to enhance the performance of the system. The same yields for the features needed to predict grasp. For some users, the anatomical y-axis (representing flexion and extension) performs better than combining all three anatomical axes. In all cases, the features regarding the relative angular velocities of segments as determined using the sensor-to-segment calibration performed better than using the norm of the angular velocity vector.

Next to the kernel and features, the performance of the algorithm can be improved by personalizing the trade-off between sensitivity and specificity. Someone that uses his hand most of the time to grasp something would benefit from a high sensitivity in order to detect every grasp. Whereas if someone still moves his hand a lot while for example talking, the person would benefit from a higher specificity, since it would be irritating to continuously make a fist gesture while moving your hand around. The system would be even more improved 
if it is possible to set the sensitivity and specificity according to the activity the user would like to perform.

For application of the grasp intention detection in wearable soft-robotic devices for support of hand function for people with hand limitations, it is needed to repeat this research with the target population. Since the degree of disability differs per patient, it could be possible that a multi-user classifier is not an option, despite the results of this research. In that case, it would be interesting to define the number of trials needed for training to see whether it is feasible to use single-user classifiers for this application.

\section{Conclusion}

In conclusion, both single-user and multi-user classifiers achieve high accuracies $(98.2 \%$ and 91.4\% respectively) in distinguishing reach-to-grasp from reach-without-grasp movements in healthy subjects. Promising results were found for the detection of grasp intention. At $70 \%$ sample length, an accuracy of $90.6 \%$ was achieved; at $40 \%$ sample length, an accuracy of $85.3 \%$ was achieved. These findings allow for a faster detection of grasp by $600 \mathrm{~ms}$ to 1200 ms (depending on the preferred accuracy). If similar results are achieved with this method in stroke patients, two IMU's could be used to control grasp-supporting devices to support hand function during daily life activities. 



\title{
Detection of the intention to grasp during reaching in stroke using inertial sensing
}

Chapter 9

\begin{abstract}
To support stroke survivors in activities of daily living, wearable soft-robotic gloves are being developed. An essential feature for use in daily life is detection of movement intent to trigger actuation without substantial delays. To increase efficacy, the intention to grasp should be detected as soon as possible, while other movements are not detected instead. Therefore, the possibilities to classify reach and grasp movements of stroke survivors, and to detect the intention of grasp movements, were investigated using inertial sensing. Hand and wrist movements of 10 stroke survivors were analyzed during reach and grasp movements using inertial sensing and a Support Vector Machine classifier. The highest mean accuracies of $96.8 \%$ and $83.3 \%$ were achieved for single- and multi-user classification respectively. Accuracies of up to $90 \%$ were achieved when using $80 \%$ of the movement length, or even only $50 \%$ of the movement length after choosing the optimal kernel per person. This would allow for an earlier detection of 300-750 ms, but at the expense of accuracy. In conclusion, inertial sensing combined with the Support Vector Machine classifier is a promising method for actuation of grasp-supporting devices to aid stroke survivors in activities of daily living. Online implementation should be investigated in future research.
\end{abstract}




\section{Introduction}

In 2013, 25.7 million people of the world population suffered a stroke [173], of which $77.4 \%$ show motor impairments of the upper extremities [7]. The impairments manifest as muscle weakness, changes in muscle tone and a decrease in motor control, which results in difficulty of successfully performing basic hand movements such as reaching, grasping objects, and holding objects [174]. Even though strategies in rehabilitation therapy exist to aid recovery, $40 \%$ of the total stroke population suffers from chronic motor impairment $[174,175]$. Stroke survivors with only one functional hand are restricted in the performance of daily life tasks like preparing meals, housework, and shopping $[175,176]$.

Stroke survivors can be supported by means of robotic orthoses, functional aids, casts, splints, biofeedback, and electrical stimulation [21, 177]. However, most of these technologies either are bulky, restrict movement or are uncomfortable [177]. The development of orthoses to support the hand during activities of daily living ( $A D L)$ is becoming increasingly prevalent [40]. The majority of them is controlled by either surface electromyography, interaction forces or human movement [40]. One developed orthosis that is slim, portable, and developed to support grip strength in ADL, is the Soft Extra Muscle (SEM) Glove ${ }^{\mathrm{TM}}$ from Bioservo Technologies $A B$ [163]. Pressure sensors measure forces during grasping, which are located at the gray circles on the distal phalanges of the thumb, middle- and ring finger when worn (Figure 9.1a). The SEM Glove ${ }^{\mathrm{TM}}$ is developed to support users in grasping and holding objects by actuation of artificial tendons to apply extra force to the object as soon as a grasping force is detected. This could make the SEM Glove ${ }^{\mathrm{TM}}$ a suitable solution for stroke survivors that do not show spasticity or contractures but still experience problems with executing grasping movements. Up to now, a comparable, further developed, version of the SEM Glove ${ }^{\mathrm{TM}}$ is the only wearable soft-robotic glove that has shown to be feasible when used independently during ADL at home by stroke patients [136].

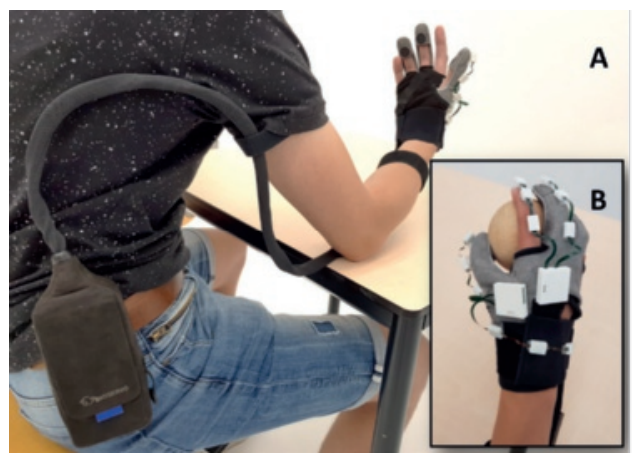

Figure 9.1 Representation of instrumentation of the experiment. A) shows the entire SEM Glove ${ }^{\mathrm{TM}}$ with pressure sensors placed on the grey circles, B) shows the SEM Glove with the white inertial measurement system. 
The pressure sensors on this glove are currently the only source of detection of grasp movements, which means that detection occurs after the subject touches the object. In a study performed by Radder et al.[109], elderly people with declined hand function performed tasks considerably faster without a comparable glove having the same pressure sensors compared with performance of the same task with glove. To increase efficacy of the SEM Glove ${ }^{\mathrm{TM}}$ to support stroke survivors in $A D L$, the intention to grasp should be detected as soon as possible while other movements, such as reaching, are distinguished from grasp movements.

The possibility to detect different final hand postures of both healthy subjects and stroke survivors was already investigated by several studies [126-128, 164, 165, 178-180]. To classify the final hand posture, bend sensors, pressure sensors, position of the fingertips, electromyography, electromagnetic sensors in combination with electromyography, and segment angles were used. In contrast to classifying different final hand postures, De Vries et al.[181] studied the possibility to distinguish reach from grasp movements while using a minimal amount of inertial sensing. In that study reach and grasp movements were classified in healthy subjects using single- and multi-user classification support vector machine (SVM) classifiers with accuracies up to $98.2 \%$ and $91.4 \%$ respectively. By using $40 \%$ of the available data of a single movement, an accuracy of $85.3 \%$ was achieved. Although at the expense of accuracy, grasp movements could be detected up to $1200 \mathrm{~ms}$ before the subject touches the object. However, stroke survivors show altered upper limb kinematic movement characteristics within a reach and grasp movement as compared to healthy subjects [182]. Therefore, as a next step, this research needs to be translated such that ultimately the prospective solution could be applied in the grip-supporting glove for stroke if proven useful. Hence, the goal of this study was to investigate the possibilities to classify reach and grasp movements of stroke survivors by analyzing their finger and hand movements using a minimal number of inertial sensors. Furthermore, the possibilities to detect a grasp movement by analyzing the intention of the movement was investigated.

\section{Methods}

\section{Participants}

Ten stroke survivors were recruited for this cross-sectional study performed at Roessingh Research and Development (RRD), Enschede, the Netherlands. Criteria for inclusion into this study were: 1) clinically diagnosed with unilateral ischemic or hemorrhagic stroke at least three months before inclusion; 2) between 18-80 years of age; 3 ) able to actively extend the fingers enough to grasp a cylindrical object with a diameter of $6 \mathrm{~cm} ; 4)$ able to actively extend the fingers enough to grasp a ball with a diameter of $7.5 \mathrm{~cm} \mathrm{5}$ ) a sufficient cognitive status to understand two-step instructions in Dutch; and 6) having (corrected to) normal vision. 
Criteria for exclusion were: 1 ) severe sensory problems or pain of the affected hand; 2 ) severe contractures limiting the passive range of motion and; 3) co-morbidities limiting functional use of the hand. The Medical Research Ethics Committee (MREC) Twente, the Netherlands, approved the study (CCMO number NL64511.044.17). All stroke survivors provided written informed consent prior to the start of this study.

\section{Instrumentation}

The pressure sensors in the three-fingered SEM Glove ${ }^{\mathrm{TM}}$ were used to detect, and determine the duration of, contact with the object in the case of a grasping movement. The thumb, middle- and ring finger are covered by the glove. The pressure sensors are located at the distal part of those fingers. Relaxation of active finger flexion enables releasing of the object. An inertial measurement system with inertial measurement units [166] was placed on the ulnar styloid, the dorsal side of the hand, and the phalanges of the thumb, index and middle finger to measure the angular velocities in order to detect flexion and extension movements of the thumb, fingers and wrist. A combination of the SEM Glove $\mathrm{T}^{\mathrm{TM}}$ and the inertial measurement system as used in the current study can be seen in Figure 9.1.

\section{Experimental set-up}

The set-up used in this study (Figure 9.2) was similar to study design of De Vries et al.[181]. The participant was seated at a table with adjustable height to make sure that the elbow of the affected side was flexed $90^{\circ}$ and aligned with the trunk. Targets were presented in five horizontal directions $\left(0^{\circ}, 45^{\circ}, 90^{\circ}, 135^{\circ}\right.$, and $\left.180^{\circ}\right)$ on locations within the active reaching range of motion of the participant. The affected hand was initially positioned in the middle of the semi-circle.

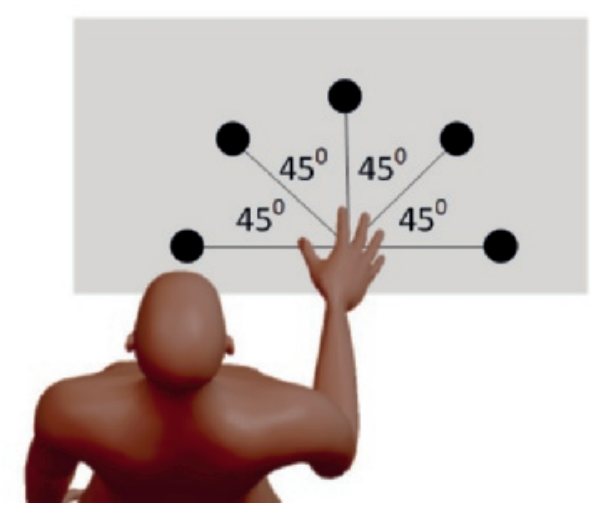

Figure 9.2 Schematic representation of the task locations for the experiment. 


\section{Protocol}

Prior to the start of the reach and grasp movements, the upper extremity part of the FuglMeyer assessment was performed to evaluate the motor status and degree of synergies in the upper limb [88]. Thereafter, anatomical rotation axes were defined with a sensor-to-segment calibration, described in the section below.

As described by De Vries et al.[181], two different grasp tasks and two different reach tasks towards the five different locations were performed: 1) grasping a wooden ball with a diameter of $7.5 \mathrm{~cm}$; 2) grasping a cylindrical object with a diameter of $6 \mathrm{~cm} ; 3$ ) reaching towards a target location whilst in pronation; and 4) reaching towards a target location whilst in supination. The grasp gestures, cylindrical and spherical, represent two common grasps in stroke [26]. The protocol allowed compensatory movements to ensure that natural movements from each individual were captured. In healthy subjects, a minimum number of 70 movements were needed to train the classifier accurately [181]. Based on the results from De Vries et al.[181], it was decided to repeat all tasks 5 times per location. Besides a specific task and location, the patient was also instructed to have one of two different starting hand postures; either flat on the table with the dorsal side upwards or making a fist with the medial side resting on the table. Therefore, a total of 100 grasp and 100 reach movements were performed of which the order of the tasks as well as the order of initial hand posture and the locations were randomized.

\section{Sensor-to-segment calibration}

A sensor-to-segment calibration as described by Luinge et al.[167] was performed to determine the anatomical rotation axes. Participants were instructed to stand upright and hold their elbow in a flexion angle of $90^{\circ}$ while the dorsal side of the hand faced upwards as reference position. Participants were then asked to perform and repeat six tasks five times: 1 ) flexion and extension of the fingers; 2 ) ab- and adduction of the fingers; 3 ) flexion and extension of the thumb; 4) flexion and extension of the wrist; 5) ab- and adduction of the wrist; 6 ) flexion and extension of the elbow. The coordinate system of each segment was defined according to the coordinate system of the whole body in anatomical position. The x-axis was defined as the anteroposterior axis pointing in anterior direction representing ab-and adduction, the $y$-axis was defined as the mediolateral axis pointing in lateral direction of the right hand representing flexion and extension, and the z-axis was the longitudinal axis pointing in caudal direction, which represents pro- and supination. The direction of the $x$-axis was determined by measuring the gravitational force at the reference position. By analyzing the direction of angular velocity during flexion of the different segments (middle finger, index finger, thumb, wrist and arm), the direction of the $y$-axis was determined. The direction of the $z$-axis was then determined by the cross-product of the $x$ - and $y$-axis. To correct for unwanted movements performed in 
the reference position and to make the coordinate system orthogonal, the direction of the $x$-axis was recalculated by computing the cross-product of the $y$ - and $z$-axis. Finally, to acquire the segment data with the coordinate system of the human body, the gyroscope data was multiplied with the rotation matrices specified by the unit vectors.

\section{Data analysis}

Data was acquired from the SEM Glove ${ }^{\mathrm{TM}}$ using Tera Term version 4.98 and from the inertial measurement system using MATLAB version 2016b on a laptop running a 64-bit Windows 10 OS with a $2.20 \mathrm{GHz}$ i7-2670QM Intel ${ }^{\circledR}$ Core $^{\mathrm{TM}} \mathrm{CPU}$ and $6 \mathrm{~GB}$ of RAM. For safety reasons, the SEM Glove ${ }^{\mathrm{TM}}$ was connected to the laptop with a USB isolator (Model UH401) from Advantech. Data analysis was performed using MATLAB version 2017b on a desktop running a 64-bit Windows 10 OS with a $3.40 \mathrm{GHz}$ i5-7500 Intel ${ }^{\circledR}$ Core $^{\mathrm{TM}} \mathrm{CPU}$ and $8 \mathrm{~GB}$ of RAM.

Pre-processing. The offset of the sensors from the inertial measurement system and the SEM Glove ${ }^{\mathrm{TM}}$ was removed from a manually determined baseline at the beginning of each recording. Throughout the baseline, no movement of the hand and no force on the SEM Glove $^{\mathrm{TM}}$ was present. After removal of the offset, data from the inertial measurement system was filtered with a $4^{\text {th }}$ order, zero-lag, low pass Butterworth filter. The cut-off frequency was set at $6 \mathrm{~Hz} .[168,169]$

Training and classification. Within the classification learner toolbox of MATLAB, the Support Vector Machine (SVM) was used for classification of the data since it is able to find patterns in high dimensional, non-linearly separable data and can accurately distinguish between two discrete classes $[170,171]$. The two defined classes in the current study were 'reach' and 'grasp' and the SVM classifiers were trained and validated using 10-fold cross-validation. Besides the standard linear boundary, the quadratic, cubic, fine Gaussian, medium Gaussian and coarse Gaussian kernels were investigated in the current study. Classification was performed in two scenarios: 1 ) by splitting the dataset of one patient into a test and training set, i.e. single-user analysis; 2 ) by splitting the dataset of all patients into a test set of one patient and a training set of other patients, i.e. multi-user analysis.

Database. The data were divided into 200 trials per subject, each trial containing a reach or grasp movement. During a reach movement, the beginning and end of a trial were determined using a threshold detector algorithm for the angular velocity (threshold $\pm 0.1 \mathrm{rad} / \mathrm{s}$ ) [181]. In case of a grasp movement, the end of a trial is defined as the moment the pressure sensors in the SEM Glove ${ }^{\mathrm{TM}}$ detected contact.

Single-user. In case of single-user classification, 25\% of the 200 trials (25 grasp and 25 reach 
trials) was randomly selected for the test set. The remaining 150 trials of the same participant were used for training.

Multi-user. Two methods of multi-user classification were performed. First, the trials of one participant were selected for the test set, while the trials of the remaining subjects were selected for the training set. Second, the participants were first divided into categories of stroke severity (mild, moderate and severe [88]) following the FMA score after which the first classification method was done for each group. For each participant, a separate database was created.

Feature extraction. The extraction of features was performed in a similar way as by De Vries et al.[181]. The movements of each segment were expressed with respect to the dorsal side of the hand by determining the relative angular velocity of a segment $s\left(\omega_{s}\right)$ in each rotation axis separately and the norm angular velocity vector of a segment $s\left(\right.$ norm $\left._{s}\right)$. The previously described sensor-to-segment calibration was used to determine the relative angular velocity, $\omega_{s^{\prime}}$ by subtracting the data of the dorsal side of the hand from the data of a segment $s$. The second parameter, norm $s^{\prime}$ was calculated by taking the norm of the difference in angular velocities on the $x^{-}, y^{-}$, and $z$-axes between a segment $s$ and the dorsal side of the hand.

Two features of the segments, the mean and standard deviation (SD), were calculated for both parameters to get eight features per segment relative to the dorsal side of the hand; two for the norm and six for the three components of the relative angular velocity. In total four segments were used to calculate the separate features: the distal part of the forearm and the distal phalanges of the thumb, index and middle finger. So in total, a number of 32 features were extracted from each trial.

Several different combinations of these features were determined to train and test the SVM classifier. To limit the number of sensors, at most two sensors were used in each feature combination: the dorsal side of the hand with one other segment. Table 9.1 shows the combinations of features used in the experiment. The first four feature combinations consist of the mean and SD of the norm angular velocity vector of the middle finger, index finger, forearm and thumb with respect to the dorsal side of the hand. Feature combinations 5 to 8 represent the mean and SD of the relative angular velocities of all axes of the described segments. Because the $y$-axis of each segment represents flexion and extension, the mean and SD of the relative angular velocity of only the $y$-axis were used as separate feature combinations, which are numbers 9 to 12. Finally, due to the saddle joint of the thumb, the mean and SD of the relative angular velocities of the $x$ - and $z$-axis of the thumb were also used as the last two feature combinations. This means that all feature combinations consist of two features per 
combination, except for combinations 5 to 8 , which contain six features each.

Grasp intention detection. The possibilities to detect a grasp movement prior to touching the object were investigated by analyzing the intention of the movement. This means that each trial was cut into various shorter lengths, varying from $10 \%$ of the total trial length to $90 \%$, in steps of $10 \%$. Then for every step the single- and multi-user classification was performed to determine the accuracies as the trial length declines.

Table 9.1 Feature combinations of the experiment. Each described feature implies the mean and SD of said feature.

\begin{tabular}{lll}
\hline$\#$ & Feature combination & Segment \\
\hline $\mathbf{1}$ & norm $_{s}$ & Middle finger \\
$\mathbf{2}$ & norm $_{s}$ & Index finger \\
$\mathbf{3}$ & norm $_{s}$ & Forearm \\
$\mathbf{4}$ & norm & Thumb \\
$\mathbf{5}$ & $\omega_{s}(x-, y$-, and $z$-axis $)$ & Middle finger \\
$\mathbf{6}$ & $\omega_{s}(x-, y-$ - and $z$-axis $)$ & Index finger \\
$\mathbf{7}$ & $\omega_{s}(x-, y-$ - and $z$-axis $)$ & Forearm \\
$\mathbf{8}$ & $\omega_{s}(x-, y-$, and $z$-axis $)$ & Thumb \\
$\mathbf{9}$ & $\omega_{s}(y$-axis $)$ & Middle finger \\
$\mathbf{1 0}$ & $\omega_{s}(y$-axis $)$ & Index finger \\
$\mathbf{1 1}$ & $\omega_{s}(y$-axis & Forearm \\
$\mathbf{1 2}$ & $\omega_{s}(y$-axis $)$ & Thumb \\
$\mathbf{1 3}$ & $\omega_{s}(x$-axis $)$ & Thumb \\
$\mathbf{1 4}$ & $\omega_{s}(z$-axis $)$ & Thumb \\
\hline
\end{tabular}

\section{Results}

\section{Participants}

Ten chronic stroke survivors were included in this study (Table 9.2). Based on a categorization of the FMA score without reflexes [92], six (60\%) of the included stroke survivors were mildly affected (FMA score $>41$ ), and four (40\%) were moderately affected ( $28 \geq$ FMA score $\geq 41)$. In six of the participants, the affected side was the dominant side pre-stroke. 
Table 9.2 Participant characteristics.

\begin{tabular}{ll}
\hline & Participants $(\mathbf{N}=\mathbf{1 0})$ \\
\hline Sex (male/female) & $5 / 5$ \\
${\text { Age }(\text { years })^{\mathrm{b}}}^{\mathrm{b}}$ & $61.0 \pm 7.6(51-74)$ \\
Time post stroke (years) $^{\mathrm{b}}$ & $5.8 \pm 2.3(2.5-10)$ \\
Affected body side (left/right) $^{\mathrm{a}}$ & $5 / 5$ \\
Dominant body side pre-stroke (left/right) $^{\mathrm{a}}$ & $1 / 9$ \\
Fugl-Meyer assessment score $^{\mathrm{b}}$ & $49.8 \pm 7.0(37-57)$ \\
\hline
\end{tabular}

${ }^{a}$ Absolute numbers, ${ }^{b}$ mean \pm standard deviation (range)

\section{Single-user classification}

Using single-user classification for all combinations of feature combination and kernel, which required on average a computing time of $175.0 \mathrm{~s}$ ( $\pm 40.4 \mathrm{~s}$ ), the highest mean accuracy of $96.8 \%$ ( $\pm 4.54 \%$ ) was achieved by the mean and SD of the relative angular velocities of all axes of the middle finger (feature combination 5) with the medium Gaussian kernel (Table 9.3). This combination of the SVM classifier was trained in $0.073 \mathrm{~s}$ on average and showed a mean sensitivity and specificity of $98.0 \%$ ( $\pm 3.37 \%)$ and $96.1 \%( \pm 6.25 \%)$ respectively. When optimizing the feature combination and kernel for each person, accuracies ranging from $96.0 \%-100 \%$ were found, with an average accuracy of $99.0 \%$.

Table 9.3 Mean accuracies and SD (\%) of combinations of feature combination and kernels for the single-user classification. Only the feature combinations where the best performing kernel per feature combination showed a mean accuracy of at least $90 \%$ are reported. The numbers of the feature combinations correspond to the described feature combinations in Table 9.1. The kernel with the highest accuracy for each feature combination is marked in bold text and the highest accuracy overall is underlined.

\begin{tabular}{lllllll}
\hline Kernel & Linear & Quadratic & Cubic & $\begin{array}{l}\text { Fine } \\
\text { Gaussian }\end{array}$ & $\begin{array}{l}\text { Medium } \\
\text { Gaussian }\end{array}$ & $\begin{array}{l}\text { Coarse } \\
\text { Gaussian }\end{array}$ \\
$\begin{array}{l}\text { Feature } \\
\text { combination }\end{array}$ & & & & & & \\
\hline 5 & $93.4 \pm 5.89$ & $95.2 \pm 7.25$ & $96.2 \pm 4.76$ & $94.6 \pm 4.22$ & $\underline{96.8} \pm \mathbf{4 . 5 4}$ & $83.0 \pm 15.4$ \\
6 & $93.0 \pm 6.06$ & $95.4 \pm 6.19$ & $\mathbf{9 4 . 8} \pm \mathbf{5 . 4 3}$ & $94.2 \pm 5.03$ & $95.2 \pm 5.67$ & $84.8 \pm 13.2$ \\
7 & $80.2 \pm 9.11$ & $\mathbf{9 0 . 4} \pm \mathbf{6 . 5 2}$ & $89.8 \pm 4.76$ & $89.0 \pm 6.62$ & $90.4 \pm 6.17$ & $76.6 \pm 9.00$ \\
8 & $88.0 \pm 9.43$ & $92.9 \pm 5.30$ & $\mathbf{9 3 . 3} \pm \mathbf{5 . 5 7}$ & $86.4 \pm 6.15$ & $90.4 \pm 7.20$ & $83.8 \pm 11.5$ \\
9 & $84.8 \pm 14.1$ & $86.0 \pm 13.4$ & $86.8 \pm 12.9$ & $\mathbf{9 0 . 6} \pm \mathbf{7 . 3 1}$ & $88.2 \pm 12.6$ & $78.6 \pm 14.8$ \\
10 & $87.6 \pm 12.9$ & $90.8 \pm 8.65$ & $90.6 \pm 9.66$ & $\mathbf{9 1 . 4} \pm \mathbf{8 . 3 3}$ & $90.8 \pm 10.4$ & $80.4 \pm 17.1$ \\
\hline
\end{tabular}




\section{Multi-user classification}

The highest mean accuracy of $83.3 \%$ ( \pm 9.99\%) for the multi-user classification for all combinations of feature combination and kernel was achieved by the mean and SD of the relative angular velocities of all axes of the middle finger (feature combination 5) with the medium Gaussian kernel (Table 9.4). This combination of the SVM classifier was trained in 0.43 S on average and showed a mean sensitivity and specificity of $87.2 \%( \pm 8.22 \%)$ and $83.0 \%$ ( \pm $12.8 \%)$ respectively. The highest mean accuracy after the categorical multi-user classification for the mildly affected stroke survivors, $85.3 \%$ ( $\pm 8.31 \%$ ), was achieved by the mean and SD of the relative angular velocities of all axes of the middle finger (feature combination 5 ) with the linear kernel and was trained in $0.5 \mathrm{~s}$ on average. For the moderately affected category, the highest accuracy was $77.4 \%$ ( $\pm 12.7 \%$ ) by the mean and SD of the relative angular velocity of the $y$-axis of the index finger (feature combination 10) with the coarse Gaussian kernel and was trained in $0.12 \mathrm{~s}$ on average.

Table 9.4 Mean accuracies and SD (\%) of combinations of feature combination and kernels for the multi-user classification. Only the feature combinations where the best performing kernel per feature combination showed a mean accuracy of at least $90 \%$ in the single-user classification are reported. The numbers of the feature combinations correspond to the described feature combinations in Table 9.1. The kernel with the highest accuracy for each feature combination is marked in bold text and the highest accuracy overall is underlined.

\begin{tabular}{lllllll}
\hline Kernel & Linear & Quadratic & Cubic & $\begin{array}{l}\text { Fine } \\
\text { Gaussian }\end{array}$ & $\begin{array}{l}\text { Medium } \\
\text { Gaussian }\end{array}$ & $\begin{array}{l}\text { Coarse } \\
\text { Gaussian }\end{array}$ \\
$\begin{array}{l}\text { Feature } \\
\text { combination }\end{array}$ & & & & & & \\
\hline 5 & $80.7 \pm 12.0$ & $82.8 \pm 11.1$ & $80.3 \pm 9.54$ & $80.0 \pm 9.09$ & $\underline{\mathbf{8 3 . 3} \pm \mathbf{9 . 9 9}}$ & $81.9 \pm 11.5$ \\
6 & $80.3 \pm 12.2$ & $79.0 \pm 13.4$ & $74.3 \pm 9.45$ & $76.1 \pm 8.97$ & $78.9 \pm 11.2$ & $\mathbf{8 2 . 3} \pm \mathbf{1 4 . 8}$ \\
7 & $61.6 \pm 7.67$ & $59.2 \pm 8.78$ & $52.5 \pm 12.5$ & $61.7 \pm 5.63$ & $61.2 \pm 9.50$ & $\mathbf{6 2 . 8} \pm \mathbf{1 0 . 3}$ \\
8 & $69.2 \pm 11.5$ & $\mathbf{7 4 . 7} \pm \mathbf{9 . 9 3}$ & $68.2 \pm 12.1$ & $67.4 \pm 10.2$ & $74.4 \pm 11.3$ & $72.7 \pm 10.7$ \\
9 & $81.1 \pm 12.8$ & $66.7 \pm 13.7$ & $50.5 \pm 7.37$ & $79.5 \pm 13.8$ & $80.2 \pm 12.8$ & $\mathbf{8 1 . 3} \pm \mathbf{1 3 . 7}$ \\
10 & $80.2 \pm 13.7$ & $80.0 \pm 14.3$ & $42.7 \pm 11.2$ & $78.7 \pm 13.4$ & $81.5 \pm 14.5$ & $\mathbf{8 2 . 0} \pm \mathbf{1 4 . 9}$ \\
\hline
\end{tabular}

\section{Grasp intention detection}

The mean accuracies and SD using the described variation of trial lengths for the single-user classification are shown in Table 9.5. Using $80 \%$ of the movement length, a mean accuracy of $87.6 \%$ ( $\pm 9.42 \%$ ) was achieved by taking the mean and SD of the relative angular velocities of all axes of the middle finger with a medium Gaussian kernel. After optimization of the kernel per person, an accuracy of $90.4 \%$ ( $\pm 6.10 \%$ ) was achieved when using only $50 \%$ of the 
movement length. The intention to grasp could be detected approximately $750 \mathrm{~ms}$ earlier with the inertial measurement units and SEM Glove ${ }^{\mathrm{TM}}$ setup combined with the SVM classifier than the SEM Glove ${ }^{\mathrm{TM}}$ using the pressure sensors when using only $50 \%$ of the movement length.

Table 9.5 Mean accuracies and SD of the relative angular velocities of all axes of the middle finger of different trial lengths for the single-user classification with the medium Gaussian kernel and the best kernel combination per person.

\begin{tabular}{|c|c|c|c|}
\hline Trial length (\%) & Kernel & Median Gaussian & Tailored fit \\
\hline 10 & & $71.0 \pm 10.6$ & $78.0 \pm 5.33$ \\
\hline 20 & & $75.6 \pm 5.87$ & $80.2 \pm 3.46$ \\
\hline 30 & & $76.0 \pm 8.79$ & $84.1 \pm 4.51$ \\
\hline 40 & & $80.6 \pm 8.75$ & $88.8 \pm 6.41$ \\
\hline 50 & & $82.8 \pm 8.44$ & $90.4 \pm 6.10$ \\
\hline 60 & & $84.0 \pm 8.89$ & $90.8 \pm 5.75$ \\
\hline 70 & & $84.2 \pm 9.35$ & $93.6 \pm 3.86$ \\
\hline 80 & & $87.6 \pm 9.42$ & $94.8 \pm 3.55$ \\
\hline 90 & & $92.6 \pm 8.49$ & $96.8 \pm 3.16$ \\
\hline
\end{tabular}

\section{Discussion}

The current study explored possibilities to classify reach and grasp movements, as well as to detect the intention to grasp, of stroke survivors by analyzing their finger, hand and wrist movements using a minimal number of inertial sensors. From the results of the experiment, it can be said that reach movements can be distinguished from grasp movements by using only two inertial measurement units: one sensor on the dorsal side of the hand and one on a distal phalange of the thumb, middle- or index finger or distal part of the forearm. By using the single-user classification method, the highest mean accuracy of $96.8 \%$ was achieved whereas the multi-user classification method achieved a highest mean accuracy of $83.3 \%$. In both cases these highest accuracies were achieved by the mean and SD of the relative angular velocities of all axes of the middle finger (feature combination 5) with the medium Gaussian kernel. After optimizing the feature combination and kernel per person, accuracies ranging from $96 \%$ to $100 \%$ were reached. Accuracies up to $90 \%$ were achieved when using $80 \%$ of the movement length by taking the mean and SD of the relative angular velocities of all axes of the middle finger with a medium Gaussian kernel, or even only $50 \%$ of the movement length after optimizing the kernel per person. This would allow for an earlier grasp detection of 300 ms ( $80 \%$ movement length) to $750 \mathrm{~ms}$ ( $50 \%$ movement length) compared to grasp detection by the pressure sensors in the original setup of the SEM Glove ${ }^{\mathrm{TM}}$. 
So far, studies distinguishing different grasps in stroke show comparable or lower accuracies (87\% [179], 89.5\% [178] and 73\% [180]), using segment angles [179] or EMG signals [178, 180] for classification. In contrast to those studies, the present study used a minimal number of two inertial sensors and distinguished between reach and grasp, instead of using multiple sensors and distinguishing between different grasps. In the work of De Vries et al.[181], singleand multi-user classification accuracies of $98.2 \%$ and $91.4 \%$ respectively were achieved when distinguishing reach from grasp in healthy participants. In the case of single-user classification, our result is similar to the results of the De Vries study, but the multi-user classification showed a substantially lower accuracy. As previously described, stroke survivors show different and more variable movement patterns as compared to healthy controls [182], which can explain that a multi-user classification method for stroke survivors is not as robust as for healthy subjects with less variable movement patterns.

Moderately affected stroke patients generally have a larger movement variability than mildly affected stroke patients [182]. When dividing the stroke participants from the present study in two categories for the multi-user classification and comparing the mean accuracies to the first grouped multi-user classification, comparable results for the mild category were achieved, but worse results were found for the moderate category. A similar trend, in which lower accuracies occurred when subjects were more severely impaired, was also found in the study of Lee et al.[180]. Altogether, for the SVM classifier to be feasible to distinguish reach from grasp of stroke survivors accurately, a single-user classification method might be the better option. Because optimizing the feature combination and kernel per person showed possible increases of the accuracy up to $100 \%$, for future developments a tailor-made system, in terms of software, should be considered. Concerning computation time, this should not propose any problems.

The results from the user dependent grasp intention detection showed that the intention to grasp could be detected $300 \mathrm{~ms}$ ( $80 \%$ movement length) to $750 \mathrm{~ms}$ ( $50 \%$ movement length) earlier than when using the SEM Glove ${ }^{\mathrm{TM}}$ in its original configuration using pressure sensors. Depending on the accuracy that is desired, grasp intention could be detected even earlier than 750 ms before the SEM Glove ${ }^{\mathrm{TM}}$ currently does by optimizing sensitivity and specificity tradeoffs individually. If a stroke survivor, for example, performs solely grasp movements with the affected hand, the system can be more sensitive, but if the movements involve a high variety in reach and grasp, the system could be set at a higher specificity.

This research showed promising results for both single- and multi-user classification to distinguish reach from grasp movements in chronic stroke survivors. The inertial measurement unit setup used in the present study is suitably small to integrate in the glove itself, if desired. 
Although the multi-user classification achieved lower accuracies than single-user classification, higher accuracies for a specific stroke survivor could be achieved by adjusting the sensitivity and specificity in a multi-user setting as well. Only two sensors were used for each separate analysis in this experiment. Although from the perspective of cost and robustness it would be preferred to incorporate no more than two sensors in the SEM Glove ${ }^{\mathrm{TM}}$, it might be that higher multi-user classification accuracies could be achieved with combinations that use more than two sensors. In the future, combinations of sensors/features such as the mean and SD of the relative angular velocity of all axes of the thumb and middle finger, could be included in the analysis using SVM to determine whether this yields generalizable results. Although adding complexity to the system, this solution might be highly advantageous when applied as intention detection method in assistive technologies, since there is no need for establishing a dataset for training the algorithm prior to use by a subject, and the system can be used in a plug-and-play manner.

All filtering and classification was performed offline in the current study, but ultimately the system should be able to function online before implementing it in the SEM Glove ${ }^{\mathrm{TM}}$. When filtering data in real-time, a latency is introduced which should be accounted for during classification. However, this latency can be minimized by choosing a suitable filter and window. Ultimately, it might be beneficial for stroke survivors to not only distinguish between reach and grasp movements, but also between different grasp movements. If the system should be able to classify between different reach and different grasp movements online using the SVM classifier, computational complexity increases and thus detection time will increase and detection accuracies will possibly decrease. Methods of using the SVM online have been proposed [183], but other analysis methods such as a Matched Filter (MF) with a threshold detection algorithm or a finite state model such as a Hidden Markov Model (HMM) [184] might be more suitable in terms of computational complexity. If the SVM classifier were to be used online, it needs to wait for the whole data sequence before doing a classification, whereas a HMM is able to update its prediction every time a part of the sequence is presented and would mean that computational load is lower [185]. A MF algorithm would only involve simplistic calculations which are easily computed. Therefore, the most appropriate method for online implementation while taking into account the computational complexity and its properties and performance for this specific application should be investigated.

\section{Conclusion}

In this study, grasp movements of stroke survivors could be accurately distinguished from reach movements using a minimal number of inertial sensors. Promising results for both single-user (96.8\%) and multi-user classification (83.3\%) were achieved. While using only part 
of the movement length, accurate grasp detection would allow for a faster grasp detection than the current method for detection in the glove. When in future research comparable results could be achieved after online implementation, inertial measurement units could be used to control devices that aid in daily life activities that involve grasp movements. 



\section{General discussion \\ Chapter 10}

\section{"How far have we reached and what have we grasped?" Alt Murphy 2015}

This thesis aimed to increase our understanding on user requirements of assistive technology (AT) in general, and on the usability and effect of specifically the grip-supporting ironHand system as an assistive device to support stroke patients in their activities of daily living, to contribute to the development of assistive technologies to support hand function of stroke patients during activities of daily living at home that meet the needs and preferences of the end-user. Based on the general objective, this thesis aimed to answer four research questions.

1. What defines the user preferences concerning assistive technology designed for the upper limb of stroke patients?

User preferences and user experience were gathered among stroke patients both before use of an AT (chapter 2 and 3 ) as well as after usage of the grip-supporting soft-robotic ironHand glove (chapter 4, 6, 7). In general, user preferences of stroke patients were defined by the wish to regain more functionality of their affected hand in daily life, regardless of the severity level of the limitations of the patients. Before use, envisaged intuitiveness and ease of use were reflected by the choice for subconscious control and receiving assistance solely as needed (chapter 3) After use of the ironHand system, user preferences were defined mainly based on usability, and intuitiveness of its use (chapter 4,6,7). Experienced intuitiveness greatly varied between participants. Our systematic review (chapter 2) revealed that several factors other than usability-related aspects, and interdependencies between those factors, affect the chance of successful implementation of an assistive device as well.

\section{Does a wearable soft-robotic glove improve task performance directly?}

The direct effect of the wearable soft-robotic glove was assessed by maximal pinch strength (chapter 6), the Jebsen-Taylor Hand function Test (JTHFT) (chapter 7), and through kinematic analyses (chapter $5 \& 6$ ). While maximal pinch strength did improve due to using the glove, a positive influence of the glove on task performance, as measured using the JTHFT and kinematic analyses, was absent. In fact, a lower task performance with glove was found, which was related solely to wearing the glove itself (non-activated). The added grip support did not influence task performance in a negative way (chapter 6).

3. Does prolonged, unsupervised, use of a wearable soft-robotic glove during ADL at home improve task performance of stroke patients? 
Despite no clear instantaneous benefit, both glove-supported and unsupported performance of the JTHFT improved after four weeks of home-use (chapter 7), highlighting the potential of the glove to support hand function during daily life activities at home without supervision.

4. Considering the limitations of pressure sensing as control input for the device, could the control of the grip-supporting glove be improved?

Fed by the findings of the preceding chapters that focused on the evaluation of usability and effectiveness of the ironHand system, chapter 8 and 9 dug deeper into potential ways to circumvent its current drawbacks and explore opportunities to harvest its assets. This showed that the intention to grasp could be detected earlier by using inertial movement sensors, instead of fingertip pressure sensors as implemented in the current version of the ironHand system. In this way, it would be possible to improve the control of the grip-supporting glove in order to ultimately improve usability and task performance.

While each chapter in this thesis provides insight into a specific topic; this discussion will combine all knowledge gained from those studies. All information taken together reveals a set of factors (needs) that are important to address, in order to contribute to the successful development and implementation of AT for use during ADL at home in stroke in a way that better meets the needs and preferences of stroke patients. We will elaborate on those factors in this discussion. In addition, the gained insights gave rise to a set of recommendations that should be considered for future research and developments of AT for home-use in general, and the ironHand system specifically, to potentially increase the chance of adoption by stroke patients.

\section{The need for a holistic approach}

To create meaningful and effective designs, the People, Activity, Context and Technologies (PACT) framework [74] and the Users Task Environment (UTE) approach [186, 187] can be used, among others, in the early design phases to cover all social and technological aspects. For the design of the ironHand system, both the PACT framework (chapter 3) and the UTE approach [26] were used to gather information about all the domains (i.e., People/User, Activities, Context/Environment, and Technology) through focus groups. In general, our insights are in line with information from existing literature that used focus groups, questionnaires and interviews to gather information about AT for the upper extremity [5, 25, 27-29]. However, only after putting all information from existing literature systematically together (chapter 2), the interdependency between identified factors (e.g., costs with usability, knowledge with motivation, familiarity and affinity with technology with trust and expected usefulness) and the importance of facilitating factors were highlighted. For example, a potential user of AT goes 
through a decision process. Prerequisite for entering the decision process is a sufficiently positive attitude towards technology and the desire to increase independence and selfmanagement of the stroke patient. The stroke patient and their carer(s) prefer to consult with a trusted healthcare professional (HCP), who may or may not have experience with AT. By combining factors such as costs, expected usefulness, and safety aspects, a decision can be reached to purchase AT. If AT incorporates therapeutic principles (such as repetition, task oriented exercises and active contribution) and can be used pleasantly in a time-efficient and safe manner, chances of acceptance increase. Time efficiency can be increased by usability factors such as setup time, clear and understandable instructions for use, easy donning or doffing, and adjustability. Features such as monitoring fatigue and detecting wrongly executed movements can contribute to safety. The interdependency of the identified themes implies that all aspects influencing user perspectives of assistive technology need to be considered when developing assistive technology to enhance its chance of acceptance. The importance of each factor may vary depending on personal factors and the use context. Although the research field of soft-robotic devices has attained considerable attention in recent years [188, 189], not all those of factors, and their interdependencies, are currently targeted, or at least not reported, in research on AT [26, 84, 124, 190].

Usually, design projects start with the elicitation of user requirements of end-users, such as stroke patients and healthcare professionals, after which those requirements are balanced with the technical possibilities and available time and money. Envisaged concepts of endusers are often not feasible from a technical point of view and constraints in time and money. Additionally, although valuable information can be gathered prior to actual use, the chance of actual use of a device is believed to be predominantly related to the experienced ease of use and perceived usefulness of the system [19,24], which cannot always be predicted beforehand. In the current research, we have assessed preferences to control a device before and after development of prototypes. When comparing users' opinions before and after actual use, we observed a remarkable mismatch. Whereas the majority of the stroke patients stated to prefer sub-conscious control beforehand (chapter 3), their opinions were more diversified after actual use (chapter 4). Users are generally having difficulties in accurately visualizing a product from an abstract concept [191], which usually is the case in the early design phases. So, we can ask ourselves what the added value of incorporating stroke patients in the conceptual phase is, especially since there is already a lot of information present in existing literature about preferences for AT coming from end-users. When users are having difficulties in visualizing a potential device, and even if users are able to express their preferences accurately; there is a significant chance that the expressed preference is not practically or technically feasible, or not as technologically advanced or innovative enough. 
The results from the systematic review (chapter 2) indicate a need for a holistic design approach when developing AT for the upper limb post stroke. In this case, holistic refers to emphasizing the importance of the whole and the interdependency of its parts. Now at completion of this thesis, with the current knowledge, we strongly suggest to address user-centered design in a new way. Instead of involving end-users (only) prior to a design to elicit (too general) user requirements, it seems more valuable to join them specifically and extensively with testing of prototypes, preferably repeatedly. Moreover, technicians from companies, clinical experts experienced with technology, and health insurance companies should be involved from the start to ensure that relevant themes and factors, such as technical feasibility, therapeutic principles, costs, and knowledge, are joined and targeted directly to start the product development.

To complement this, several models exist that examine behavioral intention to use technology, to explain how and why people adopt technologies (i.e. Theory of Reasoned Action [192], Technology Acceptance Model (TAM [193], TAM2 [194]), Theory of Planned Behavior [195], and the Unified Theory of Acceptance and Use of Technology (UTUAT) [53]). Although the UTAUT has a primary focus on IT-related technology, facilitating conditions (defined as "the degree to which an individual believes that an organizational and technical infrastructure exists to support use of the system") are incorporated as influencing factor of technology adoption. In future developments, this information can be used from the start to tailor existing models to the field of AT to ensure that all relevant factors and their interdependencies are considered.

\section{The need for customization}

To realize technology development that better suits the needs and preferences of the user, focus should not only be placed on a holistic design approach, but there is also a need for customization of AT design. There is a large variation in the experienced symptoms after stroke [7]. Recently, research has proposed cut-off points for specific clinical scales for choosing the most appropriate personal intervention, taking time post-stroke and stroke severity, such as the presence of hand movement and the presence of spasticity, into consideration [174, 196]. The fact that every patient has different needs, which should be reflected in AT, is also expressed by healthcare professionals (HCPs) and patients in the study of Elnady et al.[39]. Although challenging, advances in the field of AT are made to develop devices in such a way that these can be used in a variety of activities instead of providing support during solely one activity [84, $109,124,125,139]$. In this way, the activity in which the AT is used can be personalized towards the individual. Despite this good start, there is still a focus on the development of one-size fits all devices, and not yet on personalization to the individual needs.

Attempting to develop a device that meets the preferences of its end-users, our focus group 
(chapter 3) addressed specific and detailed aspects such as intention detection, personalization, feedback and motivational aspects. In this focus group (chapter 3), preferences for support options were depending on the degree of severity; whereas the majority preferred only assistance as needed, a more severely affected stroke patient liked to receive any kind of support. Stroke patients predominantly preferred a sub-conscious method to detect the intention of the user (e.g., eye-tracking, movement sensors or muscle activation) with the possibility to switch to, or combine it with, conscious control (e.g., voice recognition, joystick and pushing a button) to reduce the cognitive load. Subsequently, after use of the soft-robotic glove controlled by pressure, muscle activity, voice or wink in stroke (chapter 4), a high variety in personal preference to control a device was found. In a study with men with Duchenne Muscular Dystrophy was also found that subjective preference for a control modality; surface electromyography or force-based control, differed between participants. This variety in preferences was depending on the level of arm function [93]. Unfortunately, the majority of AT for the hand are currently controlled by either one of those subconscious or conscious control modalities [40]. Instead of the conventional method, where developers decide on the use of solely one control modality, a modular plug-and-play system with different options to control the device would allow for personalization based on experienced limitations and preferences, and would allow for adaptation to the situation in which a person finds himself. For example, people with more severe hand function problems might opt for an on/off signal, while mildly affected participants might prefer assist-as-needed, and people experiencing problems with their speech probably choose another modality than voice controlled grasping. Besides personalization of hardware, various data analysis techniques that can deal with the individual, such as (multilevel) longitudinal analyses [197], and machine learning based methods that can determine the existence and strength of interrelationships of various factors using neural networks [198, 199], are available for personalization.

If we continue designing one-size-fits-all devices, both disease-specific needs in the motor, sensory and cognitive domains and personal needs and preferences are not taken into account. Even though it appears to be difficult to envisage a product in the concept phase, especially early in the design phase, design choices are made, at least partly, based on those insights as gained from the end-users. Although it is time consuming and costly to involve users throughout the course of the development, the chance of developing products that fit the end-user will most likely increase if several modules can be tested interchangeably during initial testing phases. 


\section{The need for familiarization}

Besides the need for factors related to the design of an AT, attention should also be paid to the evaluation of AT since it influences further design choices, and thereby the potential of an AT to be adopted by its end-user. In the current era in which technology has become indispensable, it is not surprising that older people in particular, who do not necessarily have experience and/or affinity with technology, need some time to get used to new technological developments. It is almost self-evident that familiarization time is also necessary for daily use of AT, and should be addressed in research into the effect of AT as well. The insights gained in this thesis support this assumption (chapter 4-6).

Time needed to complete tasks, as measured during a simulated ADL task (chapter 5 and 6) and during the JTHFT (chapter 7), was not improved by the glove. Those findings are also seen in elderly and stroke patients performing ADL tasks or the JTHFT using the same threefingered glove or a five-fingered soft-robotic glove, respectively [109, 137, 200]. Besides our research group, Polygerinos et al.[84] assessed four subtasks of the JTHFT with a healthy subject using an EMG-controlled soft-robotic glove. Although the outcome of the test with the EMGcontrolled glove was compared to normative data of healthy subjects on the JTHFT, instead of a direct comparison of the same subject with and without the glove, the performance of the healthy subject with glove was found to be slower as well. According to Magiera et al.[201] it is common that, in the beginning, new ways to perform a task take more time than the old, routine, method. Radder et al.[109] showed in their study that timed performance of elderly increased across no more than 3 repetitions, within 20 minutes, of using the glove. Although they are not sure whether a plateau in performance was reached yet, this might indicate that a longer familiarization time with the glove could result in stabilizing the quality of movement execution. This idea seems plausible as we do see effects of using the glove on task performance after 4 weeks of glove use at home (chapter 7). In many cases, a (short) period of familiarization is included [202-205], however, similar to our studies did they not verify whether participants were actually familiarized. So, we are not sure if and when in a period of 30 minutes to 4 weeks all stroke patients are adequately familiarized with use of the ironHand system. Therefore, we could not say whether we measured the actual effect of the soft-robotic glove or the unfamiliarity of the participants with its use.

A specific part of sufficient familiarization for assessing the actual effect is getting acquainted with the control of an AT. In our studies, we observed a difference in preference for control modality before and after use (chapter 3 and 4). Whereas end-users predominantly opted for subconscious control before use (chapter 3 ), the conscious control modalities were ranked as preferred control modality by 4 out of 10 participants after testing the actual control methods (chapter 4). Participants in our study expressed to be having difficulties with estimating when 
the glove reacted after giving the command in some of the (more subconscious) modalities, so a participant likely got more easily acquainted with a modality that is more predictable in its response, than to the others that were more unconscious in nature. A possible explanation for the variety in user preferences could be that the opinion of stroke patients was influenced by the possibility that patients were not adequately familiarized with the control modality upon its first use when a prototype becomes available.

On top of that, the task in which the object had to be grasped and placed onto the platform should ideally be executed with the command to grasp or release being given during the reaching phase. This requires the participant to perform two tasks in parallel (moving the hand/object and producing the control command). We observed that participants performed the task in a sequential order: first reaching towards the object, and subsequently giving the command. Again, it is possible that participants were not familiarized with the task and/or the control. Besides, several studies have shown that either the cognitive or motoric task were affected by the performance of a dual task in stroke [206]. When performing two motoric tasks (e.g., walking and a bilateral tray-carrying task), either one of the dual tasks was performed worse when compared with a single task [206-208]. So besides the possibility that our participants were not familiarized with the control method or task, we could ask ourselves whether a subset of the stroke population will be capable of performing a dual task at all, even after familiarization. This is especially relevant in the case of conscious control methods, but can also play a role during control using sub-conscious control methods. So, even though the use of cross-sectional design with one measurement session can be useful, the importance of familiarization with the system prior to measuring the effectiveness needs to be stressed, as well as considering which users are suitable for which type of control taking into account cognitive ability or specifically dual tasking capacity. In later evaluation phases when the system has reached a higher maturity level, familiarization could be achieved by applying a longitudinal study design.

\section{The need for suitable outcome measures}

There is, next to the need for familiarization with an AT, also a need for suitable outcome measures when evaluating the effectiveness and usability of an AT. According to Choi et al.[209], methods for evaluating assistive technology prototypes during design have not been adequately described or evaluated. Commonly, standardized questionnaires such as the System Usability Scale (SUS) [45, 109, 137, 139, 143, 210] and intrinsic motivation inventory [109, 200, 211-213], are used to obtain subjective information on AT use. According to a user centered design, those mentioned methods are helpful to iteratively design devices [34]. However, the review (chapter 2) has highlighted the high interdependency between factors 
that affect potential adoption of AT which are not limited to usability factors only. It is crucial to consider the interdependency of factors, which influences the implementation and chance of adoption of AT from the beginning and should be intertwined with design and evaluation, rather than addressing it as a post-design activity [214]. Based on an open interview, valuable, complementary, usability information was retrieved in addition to the information of the SUS (chapter 6). For example, difficulties to don and doff the glove, and a loss of sensation were mentioned. Therefore, open interviews addressing topics specified at the design stage of the device are crucial to obtain additional information. Besides, developers should also be aware that not only the prototype of the device itself can be evaluated with users. One might even think of assessing simple aspects such as instructions for use. The instructions for use, commonly created in the wrap up phase of development when all product details are known, can have great impact on usability. Quality of the user manual can be easily improved by giving several end-users some assignments with the manual, to determine whether the device can be successfully applied by following the instructions.

Not only subjective measures but also suitable objective measures are needed to supplement the subjective findings. In this thesis, the Jebsen Taylor Hand Function Test (JTHFT) (chapter 7), pinch strength (chapter 6) and kinematics (chapter 5 and 6) are measured. Chapter 6 revealed that the soft-robotic ironHand glove, designed to support grip strength, indeed improves pinch strength of stroke patients. Especially pinch strength between thumb and middle finger increased significantly, but even pinch strength between thumb and index finger (not supported by the glove) increased on average with $15 \%$ when using the glove. These gains are in line with previous research by Prange-Lasonder et al.[139] who found, in a very small group of stroke patients $(n=4)$, an increase in pinch strength of $11-27 \%$ using the same glove, but adapted to stroke. Except for pinch strength, no beneficial direct effect of the ironHand glove on JTHFT and a simulated functional reach-and-grasp task, analyzing specifically timed performance, was found. In contrast to studies that are performed with the ironHand [109, 137], object manipulation improved with use of other soft-robotic gloves in two stroke patients [190], patients with a spinal cord injury [124] and a patient with muscular dystrophy [125]. The pilot study of Yap et al.[190] with two stroke patients showed that the performance to grasp an empty bottle and tin can (subtasks of the JTHFT) improved with their soft-robotic glove when compared to without glove. In the study of Capello et al.[124], a five-fingered glove manually controlled by the researcher with a switch was tested in one patient with a C4-C7 spinal cord injury. The score on the Toronto Rehabilitation Institute Hand Function Test (measuring unilateral gross motor function) was improved with use of the soft-robotic glove. In the study of Polygerinos et al.[125], the Box and Blocks Test (assessing fine motoric skills) of a patient with muscular dystrophy was performed better with glove than without. 
The discrepancy in the effect of a grip-supporting glove between their and our studies is likely related, at least in part, to a difference in the level of hand function of the included participants. In all three studies, participants were not able to perform the task without support while the participants in our studies were less severely impaired and were able to perform the tasks without support. It is obvious that meaningful improvements are more challenging to achieve in people that have better hand function. It is conceivable that 'meaningful improvements' will refer to different kind of improvements in this population. For instance, it is not a case of being able or being unable to perform a task, but the specific quality with which a task is performed, the endurance with a particular task or the cognitive effort it takes to complete a task, which is likely to make a difference. Therefore, in future studies concerning AT such as the soft-robotic glove, severity level should be considered carefully in the choice of appropriate outcome measures. Specifically, additional outcome measures beyond the 'traditional' function tests, addressing for instance dual tasking capacity, endurance and fatigue, or spatial and temporal aspects of movement performance, should be considered in relatively highly functioning patients.

A first exploration of such additional outcome measures was done in this research, by including kinematic analyses of movement execution, such as in chapter 5 assessing the direct effect with and without glove in the elderly population. Both a light and heavy weighted object had to be grasped and lifted onto a platform. The same experiment was performed in stroke as well, where glove worn non-activated was added as additional condition to research the transparency of the glove (chapter 6). The direct effect of the same glove was also assessed with the JTHFT (chapter 7). Movement time was not positively influenced by the glove, as measured during the JTHFT (chapter 7). While the JTHFT has a primary focus on (time needed for) task completion, other aspects necessary for task accomplishment that could be influenced by the glove are not taken into account in that outcome measure. Therefore, spatial and temporal parameters were measured during a simulated reach-and-grasp task in chapter 5 and 6 . Chapter 6 revealed that differences in movement execution (slower movements, smaller joint excursion and a larger trunk displacement) with and without glove were primarily due to wearing a glove in itself rather than receiving support from the glove. Chapter 5 and 6 also revealed that a longer time needed to execute the task was related to a longer time needed to grasp with the glove, highlighting the added value of supplementing timed performance measures, with kinematics of simulated ADL tasks.

A mismatch between subjective and objective outcomes was found in chapter 4 and 6 . In chapter 4, patients had to perform a grasp task in which the number of attempts needed to activate the glove were counted, and a timed simulated ADL task with four different control modalities: force, muscle activation, voice and wink. In addition, patients were asked to fill out 
the SUS for each control modality. In general, participants were positive about the control modalities, however, a weak correlation of subjective outcomes and objective measures was found. This mismatch of positive subjective experience not being reflected in objective outcome measures could have been the result of the lack of familiarization with each control modality, or of an inadequate choice for objective- and subjective outcome measures. As stated previously, future studies concerning AT such as the soft-robotic glove should consider severity level when choosing the appropriate outcome measure. In addition, attention should be paid to the definition of outcome measures, both subjective and objective, that reflect the added value of the device as defined by the end-user.

\section{Design of the ironHand system - moving technology towards the end- user}

In chapters 2-7, user requirements prior to use, as well as user experience and effectiveness during and after use of the ironHand glove were established and examined. Results from chapter 5 and 6 showed that grasping with the ironHand glove tends to be slower compared to without glove. This is possibly due to a delay within the system. In general participants were enthusiastic about the usability of the glove, as reflected by high SUS scores in chapter 6 and 7. Participants who were less enthusiastic were mainly disappointed by the inability to grasp smaller objects, partly because of a reduced sensation (chapter 6). The reduced sensation is likely due to wearing the glove in itself since the pressure sensors currently are located at the fingertips. This results in small objects slipping away since tactile information is of importance to maintain a secure grip while manipulating objects [146]. As a next step to increase our understanding whether the ironHand glove could better match the preferences and needs of the end-user, the possibility to use a minimal number of inertial sensors was explored to detect the intention to grasp earlier than the pressure sensors on the current ironHand system. The accuracy of using inertial sensors to distinguish reach from reach-to-grasp movements was explored offline in both healthy subjects (chapter 8) and in stroke patients (chapter 9). Promising results with accuracies over $95 \%$ were achieved when applying a user-dependent model in both healthy and stroke subjects. Although slightly lower, still promising results were found with offline multi-user classification $(91.4 \%$ and $83.3 \%$ for healthy and stroke patients, respectively). Applying a model that took individual differences into account achieved higher accuracies over a model that was based on data of all patients pooled. This further confirms the notion that personalization is important in this context, as it resulted in better results in grasp classification in particularly stroke patients.

In this thesis, the potential to improve the design of the ironHand system was addressed from a clinical point of view. From this point, engineers could continue to research the potential 
of using inertial sensors in a real-time setting. When in future research comparable results could be achieved after online implementation, inertial measurement units could be used to control devices for support of daily life activities that involve grasp movements. By that time, personal factors, such as whether participants use their hand solely to grasp or also to reach without the intention to grasp, should be taken into account to tune the sensitivity and specificity of the glove towards personal preferences. Furthermore, the intention to release should be detected subsequently before removing the pressure sensors from the glove. Another possibility would be to use movement sensors to supplement intention detection based on the pressure sensors. In that way, the delay in grasping (chapter 5 and chapter 6) could be bypassed. A negative consequence would be that the pressure sensors would still be present at the fingertips. Combining control modalities or providing a modular plug-and-play system in which several control modalities can be plugged in might fit the preferences and abilities of the end-user even better.

\section{Limitations}

One of the major limitations of this thesis is the limited sample size of our studies. This affected the statistical power. Not only the small number, but also the subset of the stroke and elderly population should be mentioned. Only chronic stroke patients were included in our studies. The time post-stroke was more than 10 years for several of them. Those patients have likely mastered several compensational strategies, including learned non-use, in those years to make sure that they can function properly in daily life. The inclusion of (sub)-acute patients might have resulted in different results. In general, 'enthusiasts' of technology did participate while this might not be representative for the entire population. As can be read throughout this thesis, the glove in its current form still suffers from several limitations. Participants knew that the glove as used during the experiments was still a prototype, but recognized the potential of the glove. It might be that participants scored the SUS based on the foreseen potential instead of solely scoring the current version of the glove. Another limitation is that although severely affected patients could probably benefit more from support from the glove in its current version, only mild to moderately affected patients could participate since more severely affected patients could not don the glove or activate the fingertip sensors. On the other hand, if severely affected patients could have donned and activated the glove, they probably would have needed support to use the arm as well. Therefore, one might even question if the stroke population is the population that benefits most from the ironHand system. Future studies should explore which patients or patient groups could benefit from the glove. Lastly, a limitation is the inclusion of only patients, carers and HCPs as stakeholders. When applying a holistic approach, not only end-users, but other stakeholders such as health insurance companies and technology companies should be taken into account as well. 


\section{Conclusions and future directions}

In order to contribute to the development of assistive technologies to support the hand of stroke patients during $A D L$ at home that meet the needs and preferences of the end-user, this thesis aimed to increase our understanding on user requirements on AT, and on the usability and effectiveness of the grip-supporting ironHand system as an assistive device to support stroke patients in their ADL. Whereas the potential of technology to support rehabilitation has been recognized before, now the potential of AT to support hand function during daily life activities at home without supervision is examined in this thesis. A point for consideration is that usually one body-side is affected, while the unaffected side can be used unobtrusively during ADL. Prerequisite for AT is therefore that it should be of great added value before stroke patients will consider to use it in daily routine. All steps taken in this thesis gradually provided more insight and gave rise to a set of recommendations that should be considered for future developments in the field of upper limb AT to be used unsupervised at home.

- Development of AT could benefit from a holistic design approach in which underlying relationships between factors, and the influence of contextual factors on the acceptance of AT, should be addressed from the beginning. At the start, stakeholders such as technicians from companies, healthcare professionals with technology experience, and people from health organizations should be involved. Involvement of stroke patients may be shifted from the start towards the stage of prototype testing.

- Strive for a plug-and-play system with the possibility to, in this particular situation, switch between various control modalities to allow testing a range to find the best (range of) option(s). We have identified a clear need for customization of (the control of) a device based on the abilities and preferences of the end-user.

- Time to familiarize with the system should be taken into account during research when testing the performance of a person with the system. Adequate familiarization is needed before assessing the effectiveness of a device.

- Strive for use of tailored outcome measures that fit the intended goal and population (e.g., level of functional limitations). In our studies, both subjective and objective outcome measures were not ideally tailored to this particular device and its population, in hindsight.

More specifically, at the moment the ironHand system is the only wearable soft-robotic device that has successfully been used unsupervised at home by stroke patients during daily activities. The potential of the glove has been shown in this thesis since both supported and 
unsupported task performance had been improved after four weeks. We have seen that stroke patients are motivated and able to use the ironHand glove independently. However, successful adoption requires more than motivation, good usability and potential, and should be targeted from a holistic point of view. Based on insights gained from this thesis, we therefore have to conclude as well that we still have much ground to cover before successfully implementing AT in the homes of stroke patients.

In the future, not only should attention be paid to design adaptations of the ironHand system, but we should also critically examine which patients could benefit most from the glove. Chronic stroke patients are usually acquainted with several compensational strategies to perform daily life activities. Sub-acute, or even acute, stroke patients could already benefit from the glove in their rehabilitation process since training intensity can be increased when the ironHand glove is used during $A D L$, even in-clinic. Besides the stroke population, people with other diagnoses affecting grip strength might benefit from the glove as well.

As shown in this discussion, there are many possibilities to further explore and enhance the development of the wearable soft-robotic ironHand system. However, the success of a product depends on the acceptance by its users. This process of acceptance starts already with familiarity of technology and their knowledge about AT. Even for AT to be used at home, the role of HCPs is crucial, since stroke patients depend on the information about AT they retrieve from their HCP. Whereas patients are searching for evidence on a case-by-case base, HCPs are reluctant to give information about AT of which no evidence-based information on the effect is present. Therefore, it would be ideal to have the ironHand system in innovative rehabilitation environments such as the Roessingh Novalab [215]. In this way, patients will have the opportunity to try out, and get acquainted with, the device, in terms of donning/doffing and other usability aspects, without putting therapists in the position to let patients purchase a device which is not proven to be effective. Even though costs are important in the decision process, patients are willing to spend money on an AT when it works for them. Furthermore, if the ironHand system can be tuned to a modular plug-and-play system, patients have the opportunity to find out which control strategy will fit them. In addition, use of the ironHand does not have to be limited to the stroke population only in such a rehabilitation environment as the Roessingh Novalab. Ultimately, this will lead to successful implementation and adoption of the ironHand glove in the homes of patients who will benefit most from the grip support. 



\section{References}

1. Go AS. Heart disease and stroke statistics - 2013 update: a report from the American Heart Association (vol 127, pg e6, 2013). Circulation 2013; 127(23).

2. Hong KS, Saver JL. Quantifying the value of stroke disability outcomes: WHO global burden of disease project disability weights for each level of the modified Rankin Scale. Stroke. 2009;40(12):3828-33.

3. Van Duin C. Demographic projections 2008-2050 [in Dutch: Bevolkingsprognose 2008-2050: naar 17,5 miljoen inwoners]. CBS Bevolkingstrend. 2009; Den Haag/Heerlen, the Netherlands.

4. Bots ML, Buddeke J, van Dis I, Vaartjes I, Visseren FLJ. Cardiovascular diseases in the Netherlands 2017 (in Dutch: Hart- en vaatziekten in Nederland 2017). the Dutch Heart Foundation (in Dutch: Nederlandse Hartstichting). 2017; Den Haag, the Netherlands.

5. Hughes A-M, Burridge JH, Demain SH, Ellis-Hill C, Meagher C, Tedesco-Triccas L, et al. Translation of evidence-based assistive technologies into stroke rehabilitation: users' perceptions of the barriers and opportunities. BMC health services research. 2014;14(1):124.

6. Truelsen T, Ekman M, Boysen G. Cost of stroke in Europe. European Journal of Neurology. 2005;12(s1):78-84.

7. Lawrence ES, Coshall C, Dundas R, Stewart J, Rudd AG, Howard R, et al. Estimates of the prevalence of acute stroke impairments and disability in a multiethnic population. Stroke. 2001;32(6):1279-84.

8. Nelles $G$, Jentzen W, Jueptner M, Müller $\mathrm{S}$, Diener H. Arm training induced brain plasticity in stroke studied with serial positron emission tomography. Neuroimage. 2001;13(6):1146-54.

9. Fisher BE, Sullivan KJ. Activity-dependent factors affecting poststroke functional outcomes. Topics in stroke rehabilitation. 2001;8(3):31-44.

10. Prange GB, Jannink MJ, Groothuis-Oudshoorn CG, Hermens HJ, IJzerman MJ. Systematic review of the effect of robot-aided therapy on recovery of the hemiparetic arm after stroke. Journal of Rehabilitation Research \& Development. 2006;43(2):171-84.

11. Kwakkel G, Kollen BJ, van der Grond J, Prevo AJ. Probability of regaining dexterity in the flaccid upper limb: impact of severity of paresis and time since onset in acute stroke. Stroke. 2003;34(9):2181-6.

12. Maciejasz P, Eschweiler J, Gerlach-Hahn K, Jansen-Troy A, Leonhardt S. A survey on robotic devices for upper limb rehabilitation. Journal of neuroengineering and rehabilitation. 2014;11(1):3.

13. Nichols-Larsen DS, Clark P, Zeringue A, Greenspan A, Blanton S. Factors influencing stroke survivors' quality of life during subacute recovery. Stroke. 2005;36(7):1480-4.

14. Taub E, Uswatte G, Mark V, Morris D. The learned nonuse phenomenon: implications for rehabilitation. Eura Medicophys. 2006;42:241-55.

15. Veerbeek JM, van Wegen E, van Peppen R, van der Wees PJ, Hendriks E, Rietberg $M$, et al. What is the evidence for physical therapy poststroke? A systematic review and meta-analysis. PloS one. 2014;9(2):e87987.

16. Schaechter JD. Motor rehabilitation and brain plasticity after hemiparetic stroke. Progress in neurobiology. 2004;73(1):61-72.

17. Kwakkel G. Impact of intensity of practice after stroke: issues for consideration. Disability and rehabilitation. 2006;28(13-14):823-30.

18. Heo P, Gu GM, Lee S-J, Rhee K, Kim J. Current hand exoskeleton technologies for rehabilitation and assistive engineering. International Journal of Precision Engineering and Manufacturing. 2012;13(5):807-24.

19. Balasubramanian S, Klein J, Burdet E. Robot-assisted rehabilitation of hand function. Current opinion in neurology. 2010;23(6):661-70.

20. Veerbeek JM, Langbroek-Amersfoort AC, Van Wegen EE, Meskers CG, Kwakkel G. Effects of robot-assisted therapy for the upper limb after stroke: a systematic review and meta-analysis. 
Neurorehabilitation and neural repair. 2017;31(2):107-21.

21. Farmer SE, Durairaj V, Swain I, Pandyan AD. Assistive technologies: can they contribute to rehabilitation of the upper limb after stroke? Archives of Physical Medicine and Rehabilitation. 2014;95(5):968-85.

22. Romer G, Stuyt HJ, Peters A. Cost-savings and economic benefits due to the assistive robotic manipulator (ARM). Proceedings of the $9^{\text {th }}$ IEEE International Conference on Rehabilitation Robotics (ICORR), Chicago, United States, 2005 June 28- July 1; p. 201-204.

23. Maheu V, Archambault PS, Frappier J, Routhier F. Evaluation of the JACO robotic arm: Clinicoeconomic study for powered wheelchair users with upper-extremity disabilities. Proceedings of the $12^{\text {th }}$ IEEE International Conference on Rehabilitation Robotics (ICORR), Zurich, Switzerland, 2011 June 27- July 1; p. 1-5.

24. Prange GB, Smulders LC, Van Wijngaarden J, Lijbers G, Nijenhuis S, Veltink P, et al.. User requirements for assistance of the supporting hand in bimanual daily activities via a robotic glove for severely affected stroke patients. Proceedings of the $14^{\text {th }}$ IEEE International Conference on Rehabilitation Robotics (ICORR), Nanyang Technological University, Singapore, 2015 Aug 11-14; p. 357-361.

25. Demain S, Burridge J, Ellis-Hill C, Hughes A-M, Yardley L, Tedesco-Triccas L, et al. Assistive technologies after stroke: self-management or fending for yourself? A focus group study. BMC health services research. 2013;13(1):334.

26. Radder B, Kottink A, Van Der Vaart N, Oosting D, Buurke J, Nijenhuis SM, et al. User-centred input for a wearable soft-robotic glove supporting hand function in daily life. Proceedings of the $14^{\text {th }}$ IEEE International Conference on Rehabilitation Robotics (ICORR), Nanyang Technological University, Singapore, 2015 Aug 11-14; p. 502-507.

27. Sivan $M$, Gallagher J, Holt $R$, Weightman A, Levesley $M$, Bhakta B. Investigating the international classification of functioning, disability, and health (ICF) framework to capture user needs in the concept stage of rehabilitation technology development. Assistive Technology. 2014;26(3):164-73.

28. Nasr N, Leon B, Mountain G, Nijenhuis SM, Prange G, Sale P, et al. The experience of living with stroke and using technology: opportunities to engage and co-design with end users. Disability and rehabilitation: assistive technology. 2016;11(8):653-60.

29. Hochstenbach-Waelen A, Seelen HA. Embracing change: practical and theoretical considerations for successful implementation of technology assisting upper limb training in stroke. Journal of neuroengineering and rehabilitation. 2012;9(1):52.

30. Lam MY, Tatla SK, Lohse KR, Shirzad N, Hoens AM, Miller KJ, et al. Perceptions of technology and its use for therapeutic application for individuals with hemiparesis: findings from adult and pediatric focus groups. JMIR rehabilitation and assistive technologies. 2015;2(1).

31. Phillips $B$, Zhao H. Predictors of assistive technology abandonment. Assistive technology. 1993;5(1):36-45.

32. Hung $\mathrm{Y}-\mathrm{H}$, Chen P-J, Lin W-Z. Design factors and opportunities of rehabilitation robots in upper-limb training after stroke. Proceedings of the $14^{\text {th }}$ International Conference on Ubiquitous Robots and Ambient Intelligence (URAI), Jeju, Korea, 2017 Jun 28- Jul 1; p. 650654.

33. Norman DA, Draper SW. User centered system design: New perspectives on human-computer interaction: CRC Press; 1986.

34. Abras C, Maloney-Krichmar D, Preece J. User-centered design. Bainbridge, W Encyclopedia of Human-Computer Interaction Thousand Oaks: Sage Publications. 2004;37(4):445-56.

35. Preece J, Rogers $Y$, Sharp H. Interaction design: beyond human-computer interaction: John Wiley \& Sons; 2015.

36. Sharples S, Martin J, Lang A, Craven M, O'Neill S, Barnett J. Medical device design in context: A model of user-device interaction and consequences. Displays. 2012;33(4-5):221-32.

37. Eger A, Bonnema G, Lutters D, van der Voort M, Productontwerpen. Lemma, 2006. ISBN: 978905931553.

38. Martin J, Norris B, Murphy E, Crowe J. Design for patient safety: User testing in the development of medical devices. London: National Patient Safety Agency. 2010. 
39. Elnady A, Mortenson WB, Menon C. Perceptions of Existing Wearable Robotic Devices for Upper Extremity and Suggestions for Their Development: Findings From Therapists and People With Stroke. JMIR rehabilitation and assistive technologies. 2018;5(1).

40. Bos RA, Haarman CJ, Stortelder T, Nizamis K, Herder JL, Stienen AH, et al. A structured overview of trends and technologies used in dynamic hand orthoses. Journal of neuroengineering and rehabilitation. 2016;13(1):62.

41. Latham NK, Jette DU, Coster W, Richards L, Smout RJ, James RA, et al. Occupational therapy activities and intervention techniques for clients with stroke in six rehabilitation hospitals. American Journal of Occupational Therapy. 2006;60(4):369-78.

42. Reinkensmeyer DJ, Bonato P, Boninger ML, Chan L, Cowan RE, Fregly BJ, et al. Major trends in mobility technology research and development: overview of the results of the NSF-WTEC European study. Journal of neuroengineering and rehabilitation. 2012;9(1):22.

43. van der Linden J, Waights V, Rogers Y, Taylor C. A blended design approach for pervasive healthcare: bringing together users, experts and technology. Health informatics journal. 2012;18(3):212-8.

44. Sivan M, Gallagher J, Makower S, Keeling D, Bhakta B, O'Connor RJ, et al. Home-based Computer Assisted Arm Rehabilitation (hCAAR) robotic device for upper limb exercise after stroke: results of a feasibility study in home setting. Journal of neuroengineering and rehabilitation. 2014;11(1):163.

45. Nijenhuis SM, Prange GB, Amirabdollahian F, Sale P, Infarinato F, Nasr N, et al. Feasibility study into self-administered training at home using an arm and hand device with motivational gaming environment in chronic stroke. Journal of neuroengineering and rehabilitation. 2015;12(1):89.

46. Lu EC, Wang RH, Hebert D, Boger J, Galea MP, Mihailidis A. The development of an upper limb stroke rehabilitation robot: identification of clinical practices and design requirements through a survey of therapists. Disability and Rehabilitation: Assistive Technology. 2011;6(5):420-31.

47. Bradley C, McGowan J, Michelson D. How does homelessness affect parenting behaviour? A systematic critical review and thematic synthesis of qualitative research. Clinical child and family psychology review. 2018;21(1):94-108.

48. Critical appraisal skills programme (CASP). Qualitative research: Appraisal tool. 10 Questions to help you make sense of qualitative research. Oxford: Public Health Resource Unit. 2014.

49. Walsh D, Downe S. Meta-synthesis method for qualitative research: a literature review. Journal of advanced nursing. 2005;50(2):204-11.

50. Thomas J, Harden A. Methods for the thematic synthesis of qualitative research in systematic reviews. BMC medical research methodology. 2008;8(1):45.

51. Cook AM, Polgar JM, Livingston NJ. Need-and task-based design and evaluation. Design and Use of Assistive Technology: Springer; 2011. p. 41-8.

52. Batavia A, Hammer G. Consumer criteria for evaluating assistive devices: Implications for technology transfer. Proceedings of the 12th annual Conference on Rehabilitation technology; 1989.

53. Venkatesh V, Morris MG, Davis GB, Davis FD. User acceptance of information technology: Toward a unified view. MIS quarterly. 2003:425-78.

54. Brewer BR, McDowell SK, Worthen-Chaudhari LC. Poststroke upper extremity rehabilitation: a review of robotic systems and clinical results. Topics in stroke rehabilitation. 2007;14(6):2244.

55. Intercollegiate Stroke Working Party. National clinical guideline for stroke 4th edition. London: Royal College of Physicians. 2012.

56. Burridge $\mathrm{JH}$, Hughes A-M. Potential for new technologies in clinical practice. Current opinion in neurology. 2010;23(6):671-7.

57. Lum P, Reinkensmeyer D, Mahoney R, Rymer WZ, Burgar C. Robotic devices for movement therapy after stroke: current status and challenges to clinical acceptance. Topics in stroke rehabilitation. 2002;8(4):40-53.

58. Money AG, Barnett J, Kuljis J, Craven MP, Martin JL, Young T. The role of the user within the 
medical device design and development process: medical device manufacturers' perspectives. BMC medical informatics and decision making. 2011;11(1):15.

59. Cherry COB, Chumbler NR, Richards K, Huff A, Wu D, Tilghman LM, et al. Expanding stroke telerehabilitation services to rural veterans: a qualitative study on patient experiences using the robotic stroke therapy delivery and monitoring system program. Disability and Rehabilitation: Assistive Technology. 2017;12(1):21-7.

60. Davis FD. User acceptance of information technology: system characteristics, user perceptions and behavioral impacts. International journal of man-machine studies. 1993;38(3):475-87.

61. Herder JL, Vrijlandt N, Antonides T, Cloosterman M, Mastenbroek PL. Principle and design of a mobile arm support for people with muscular weakness. Journal of rehabilitation research and development. 2006;43(5):591.

62. Kooren PN, Dunning AG, Janssen MM, Lobo-Prat J, Koopman BF, Paalman Ml, et al. Design and pilot validation of A-gear: a novel wearable dynamic arm support. Journal of neuroengineering and rehabilitation. 2015;12(1):83.

63. Dunning A, Herder J. A review of assistive devices for arm balancing. Proceedings of the $13^{\text {th }}$ IEEE International Conference on Rehabilitation Robotics (ICORR), Seatlle, United States, 2013 June 24-26; p. 1-6.

64. Van der Heide LA, van Ninhuijs B, Bergsma A, Gelderblom GJ, van der Pijl DJ, de Witte LP. An overview and categorization of dynamic arm supports for people with decreased arm function. Prosthetics and orthotics international. 2014;38(4):287-302.

65. Frappier J. Clinico-economic study of the JACO robotic arm for powered wheelchair users with upper-extremity disabilities. by Data4Actions Inc for Kinova, Canada. 2011.

66. Gitlin LN. Why older people accept or reject assistive technology. Generations. 1995;19:41-6.

67. Haworth R, Dunscombe S, Nichols PJR. Mobile arm supports: an evaluation. Rheumatology. 1978;17(4):240-4.

68. Kumar A, Phillips MF. Use of powered mobile arm supports by people with neuromuscular conditions. Journal of rehabilitation research and development. 2013;50(1):61.

69. Landsberger S, Leung P, Vargas V, Shaperman J, Baumgarten J, Yasuda L, et al. Mobile arm supports: history, application, and work in progress. Topics in Spinal Cord Injury Rehabilitation. 2005;11(2):74-94.

70. Stehle C, Albrecht-Buehler C. Developing more desirable products for stroke survivors. Topics in stroke rehabilitation. 2008;15(2):109-17.

71. He J, Koeneman E, Schultz R, Herring D, Wanberg J, Huang $H$, et al.. RUPERT: a device for robotic upper extremity repetitive therapy. Proceedings of the $27^{\text {th }}$ IEEE Annual International Conference on Engineering in Medicine and Biology Society (EMBC); Shanghai, China, 2005 Sep 1-4; p. 6844- 6847.

72. Yasuda YL, Bowman K, Hsu JD. Mobile arm supports: criteria for successful use in muscle disease patients. Archives of physical medicine and rehabilitation. 1986;67(4):253-6.

73. Shakya Y, Johnson MJ. A mobile robot therapist for under-supervised training with robot/ computer assisted motivating systems. Proceedings of the $30^{\text {th }}$ IEEE Annual International Conference on Engineering in Medicine and Biology Society (EMBC); Vancouver, Canada, 2008 Aug 20-24; p. 4511-4514.

74. Benyon D, Turner P, Turner S. Designing interactive systems: People, activities, contexts, technologies: Pearson Education; 2005.

75. Boynton PM. Administering, analysing, and reporting your questionnaire. Bmj. 2004;328(7452):1372-5.

76. Boynton PM, Greenhalgh T. Selecting, designing, and developing your questionnaire. Bmj. 2004;328(7451):1312-5.

77. Clegg D, Barker R. Case method fast-track: a RAD approach: Addison-Wesley Longman Publishing Co., first edition. November 1994.

78. van Ommeren AL, Prange-Lasonder G, Rietman JS, Veltink PH, Buurke J. Preliminary extraction of themes from a review about user perspectives on assistive technology for the upper limb after stroke. Converging Clinical and Engineering Research on Neurorehabilitation II: Springer; 2017. p. 323-7. 
79. Mahncke HW, Bronstone A, Merzenich MM. Brain plasticity and functional losses in the aged: scientific bases for a novel intervention. Progress in brain research. 2006;157:81-109.

80. Bullock IM, Zheng JZ, De La Rosa S, Guertler C, Dollar AM. Grasp frequency and usage in daily household and machine shop tasks. IEEE transactions on haptics. 2013;6(3):296-308.

81. Lum PS, Godfrey SB, Brokaw EB, Holley RJ, Nichols D. Robotic approaches for rehabilitation of hand function after stroke. American journal of physical medicine \& rehabilitation. 2012;91(11):S242-S54.

82. Meng Q, Xiang S, Yu H. Soft Robotic Hand Exoskeleton Systems: Review and Challenges Surrounding the Technology. 2017.

83. van Ommeren AL, Smulders LC, Prange-Lasonder GB, Buurke JH, Veltink PH, Rietman JS. Assistive Technology for the Upper Extremities After Stroke: Systematic Review of Users' Needs. JMIR rehabilitation and assistive technologies. 2018;5(2):e10510.

84. Polygerinos P, Galloway KC, Sanan S, Herman M, Walsh CJ. EMG controlled soft robotic glove for assistance during activities of daily living. Proceedings of the $14^{\text {th }}$ IEEE International Conference on Rehabilitation Robotics (ICORR), Nanyang Technological University, Singapore, 2015 Aug 11-14; p. 55-60.

85. Pandyan AD, Hermens HJ, Conway BA. Clinical Management of Spasticity and Contractures in Stroke. Neurological Rehabilitation: Spasticity and Contractures in Clinical Practice and Research: CRC Press; 2018.

86. Quigley M, Conley K, Gerkey B, Faust J, Foote T, Leibs J, et al.. ROS: an open-source Robot Operating System. ICRA workshop on open source software, Kobe, Japan, 2009 May 17; p. 5.

87. Noronha B, Dziemian S, Zito GA, Konnaris C, Faisal AA. "Wink to grasp"-comparing eye, voice \& EMG gesture control of grasp with soft-robotic gloves. Proceedings of the $15^{\text {th }}$ IEEE International Conference on Rehabilitation Robotics (ICORR), Londen, United Kingdom, 2017 July 17-20 11-14; p. 1043-1048.

88. Fugl-Meyer AR, Jääskö L, Leyman I, Olsson S, Steglind S. The post-stroke hemiplegic patient. 1. a method for evaluation of physical performance. Scandinavian journal of rehabilitation medicine. 1975;7(1):13-31.

89. Bangor A, Kortum PT, Miller JT. An empirical evaluation of the system usability scale. Intl Journal of Human-Computer Interaction. 2008;24(6):574-94.

90. Wessels R, Witte LD. Reliability and validity of the Dutch version of QUEST 2.0 with users of various types of assistive devices. Disability and rehabilitation. 2003;25(6):267-72.

91. Bangor A, Kortum P, Miller J. Determining what individual SUS scores mean: Adding an adjective rating scale. Journal of usability studies. 2009;4(3):114-23.

92. Woytowicz EJ, Rietschel JC, Goodman RN, Conroy SS, Sorkin JD, Whitall J, et al. Determining levels of upper extremity movement impairment by applying a cluster analysis to the FuglMeyer assessment of the upper extremity in chronic stroke. Archives of physical medicine and rehabilitation. 2017;98(3):456-62.

93. Lobo-Prat J, Nizamis K, Janssen MM, Keemink AQ, Veltink PH, Koopman BF, et al. Comparison between sEMG and force as control interfaces to support planar arm movements in adults with Duchenne: a feasibility study. Journal of neuroengineering and rehabilitation. 2017;14(1):73.

94. Essers B, Meyer S, De Bruyn N, Van Gils A, Boccuni L, Tedesco Triccas L, et al. Mismatch between observed and perceived upper limb function: an eye-catching phenomenon after stroke. Disability and rehabilitation. 2018:1-7.

95. Cruz-Jentoft AJ, Baeyens JP, Bauer JM, Boirie Y, Cederholm T, Landi F, et al. Sarcopenia: European consensus on definition and diagnosisReport of the European Working Group on Sarcopenia in Older PeopleA. J. Cruz-Gentoft et al. Age and ageing. 2010;39(4):412-23.

96. Dahaghin S, Bierma-Zeinstra SM, Ginai A, Pols H, Hazes J, Koes B. Prevalence and pattern of radiographic hand osteoarthritis and association with pain and disability (the Rotterdam study). Annals of the rheumatic diseases. 2005;64(5):682-7.

97. Horsten NC, Ursum J, Roorda LD, van Schaardenburg D, Dekker J, Hoeksma AF. Prevalence of hand symptoms, impairments and activity limitations in rheumatoid arthritis in relation to disease duration. Journal of rehabilitation medicine. 2010;42(10):916-21.

98. Bagis S, Sahin G, Yapici Y, Cimen OB, Erdogan C. The effect of hand osteoarthritis on grip 
and pinch strength and hand function in postmenopausal women. Clinical rheumatology. 2003;22(6):420-4.

99. Estes JP, Bochenek C, Fassler P. Osteoarthritis of the fingers. Journal of hand therapy: official journal of the American Society of Hand Therapists. 2000;13(2):108-23.

100. Nordenskiöld $U$, Grimby G. Assessments of disability in women with rheumatoid arthritis in relation to grip force and pain. Disability and rehabilitation. 1997;19(1):13-9.

101. Semble EL, Loeser RF, Wise CM. Therapeutic exercise for rheumatoid arthritis and osteoarthritis. Semin Arthritis Rheum. 1990;20(1):32-40.

102. Taekema DG, Gussekloo J, Maier AB, Westendorp RG, de Craen AJ. Handgrip strength as a predictor of functional, psychological and social health. A prospective population-based study among the oldest old. Age and ageing. 2010;39(3):331-7.

103. Shipham I. Rheumatoid arthritis: hand function, activities of daily living, grip strength and essential assistive devices. Curationis. 2003;26(3):98-106.

104. Weening-Dijksterhuis E, de Greef MH, Scherder EJ, Slaets JP, van der Schans CP. Frail institutionalized older persons: A comprehensive review on physical exercise, physical fitness, activities of daily living, and quality-of-life. American journal of physical medicine $\&$ rehabilitation. 2011;90(2):156-68.

105. Hoenig H, Taylor Jr DH, Sloan FA. Does assistive technology substitute for personal assistance among the disabled elderly? American Journal of Public Health. 2003;93(2):330-7.

106. Radder B, Prange-Lasonder GB, Kottink Al, Holmberg J, Sletta K, Van Dijk M, et al. The effect of a wearable soft-robotic glove on motor function and functional performance of older adults. Assistive technology. 2018:1-7.

107. Radder B, Prange-Lasonder G, Kottink A, Gaasbeek L, Holmberg J, Melendez-Calderon A, et al. User acceptance of a therapeutic system that enables hand training exercises in a motivating environment. Converging Clinical and Engineering Research on Neurorehabilitation II: Springer; 2017. p. 1251-6.

108. Radder B, Prange-Lasonder G, Kottink A, Gaasbeek L, Sletta K, Holmberg J, et al. Preliminary evaluation of a wearable soft-robotic glove supporting grip strength in ADL. Converging Clinical and Engineering Research on Neurorehabilitation II: Springer; 2017. p. 1245-50.

109. Radder B, Prange-Lasonder GB, Kottink Al, Gaasbeek L, Holmberg J, Meyer T, et al. A wearable soft-robotic glove enables hand support in ADL and rehabilitation: a feasibility study on the assistive functionality. Journal of Rehabilitation and Assistive Technologies Engineering. 2016;3:2055668316670553.

110. Aprile I, Rabuffetti M, Padua L, Di Sipio E, Simbolotti C, Ferrarin M. Kinematic analysis of the upper limb motor strategies in stroke patients as a tool towards advanced neurorehabilitation strategies: a preliminary study. BioMed research international. 2014;2014.

111. McCrea PH, Eng JJ, Hodgson AJ. Biomechanics of reaching: clinical implications for individuals with acquired brain injury. Disability and rehabilitation. 2002;24(10):534-41.

112. Subramanian SK, Yamanaka J, Chilingaryan G, Levin MF. Validity of movement pattern kinematics as measures of arm motor impairment poststroke. Stroke. 2010;41(10):2303-8.

113. Rantanen $T$, Avlund $K$, Suominen $H$, Schroll M, Frändin K, Pertti E. Muscle strength as a predictor of onset of ADL dependence in people aged 75 years. Aging clinical and experimental research. 2002;14(3 Suppl):10-5.

114. Giampaoli S, Ferrucci L, Cecchi F, Lo Noce C, Poce A, Dima F, et al. Hand-grip strength predicts incident disability in non-disabled older men. Age and ageing. 1999;28(3):283-8.

115. Fess EE. Grip Strength. $2^{\text {nd }}$ edition Chicago: American Society of Hand Therapists. 1992.

116. Wu G, Van der Helm FC, Veeger HD, Makhsous M, Van Roy P, Anglin C, et al. ISB recommendation on definitions of joint coordinate systems of various joints for the reporting of human joint motion-Part II: shoulder, elbow, wrist and hand. Journal of biomechanics. 2005;38(5):981-92.

117. Alt Murphy M, Willén $C$, Sunnerhagen KS. Movement kinematics during a drinking task are associated with the activity capacity level after stroke. Neurorehabilitation and neural repair. 2012;26(9):1106-15.

118. Alt Murphy $M$, Willén $C$, Sunnerhagen KS. Kinematic variables quantifying upper-extremity 
performance after stroke during reaching and drinking from a glass. Neurorehabilitation and neural repair. 2011;25(1):71-80.

119. Nijenhuis SM, Prange GB, Stienen A, Buurke J, Rietman JS. Direct effect of a dynamic wrist and hand orthosis on reach and grasp kinematics in chronic stroke. Proceedings of the $14^{\text {th }}$ IEEE International Conference on Rehabilitation Robotics (ICORR), Nanyang Technological University, Singapore, 2015 Aug 11-14; p. 404-409.

120. DeJong SL, Birkenmeier RL, Lang CE. Person-specific changes in motor performance accompany upper extremity functional gains after stroke. Journal of applied biomechanics. 2012;28(3):304-16.

121. Kamper DG, McKenna-Cole AN, Kahn LE, Reinkensmeyer DJ. Alterations in reaching after stroke and their relation to movement direction and impairment severity. Archives of physical medicine and rehabilitation. 2002;83(5):702-7.

122. Bender R, Lange S. Adjusting for multiple testing-when and how? Journal of clinical epidemiology. 2001;54(4):343-9.

123. Sallinen J, Stenholm S, Rantanen T, Heliövaara M, Sainio P, Koskinen S. Hand-grip strength cut points to screen older persons at risk for mobility limitation. Journal of the American Geriatrics Society. 2010;58(9):1721-6.

124. Cappello L, Meyer JT, Galloway KC, Peisner JD, Granberry R, Wagner DA, et al. Assisting hand function after spinal cord injury with a fabric-based soft robotic glove. Journal of neuroengineering and rehabilitation. 2018;15(1):59.

125. Polygerinos P, Galloway KC, Savage E, Herman M, O'Donnell K, Walsh CJ. Soft robotic glove for hand rehabilitation and task specific training. Proceedings of the 2005 IEEE International Conference on Robotics and Automation (ICRA), Seatlle, Washington, 2015 May 26-30; p. 2913-2919.

126. De Souza R, El-Khoury S, Santos-Victor J, Billard A. Recognizing the grasp intention from human demonstration. Robotics and Autonomous Systems. 2015;74:108-21.

127. Ekvall S, Kragic D. Grasp recognition for programming by demonstration. Proceedings of the 2005 IEEE International Conference on Robotics and Automation (ICRA), Barcelona, Spain, 2005 Apr 18-22; $p$ 748-753.

128. Naish KR, Reader AT, Houston-Price C, Bremner AJ, Holmes NP. To eat or not to eat? Kinematics and muscle activity of reach-to-grasp movements are influenced by the action goal, but observers do not detect these differences. Experimental brain research. 2013;225(2):261-75.

129. Prasad SM, Maniar HS, Soper NJ, Damiano Jr RJ, Klingensmith ME. The effect of robotic assistance on learning curves for basic laparoscopic skills. The American journal of surgery. 2002;183(6):702-7.

130. Shumway-Cook A and Woollacott MH. Motor Control: Translating Research Into Clinical Practice. 3 ed. Philadelphia: Lippincott Williams \& Wilkins, 2007.

131. Ye Y, Ma L, Yan T, Liu H, Wei X, Song R. Kinetic measurements of hand motor impairments after mild to moderate stroke using grip control tasks. Journal of neuroengineering and rehabilitation. 2014;11(1):84.

132. Kwakkel G, Kollen BJ, Krebs HI. Effects of robot-assisted therapy on upper limb recovery after stroke: a systematic review. Neurorehabilitation and neural repair. 2008;22(2):111-21.

133. Mehrholz J, Pohl M, Platz T, Kugler J, Elsner B. Electromechanical and robot-assisted arm training for improving activities of daily living, arm function, and arm muscle strength after stroke. Cochrane Database of Systematic Reviews. 2015.

134. Lambercy O, Dovat L, Yun H, Wee SK, Kuah CW, Chua KS, et al. Effects of a robot-assisted training of grasp and pronation/supination in chronic stroke: a pilot study. Journal of neuroengineering and rehabilitation. 2011;8(1):63.

135. Takahashi CD, Der-Yeghiaian L, Le V, Motiwala RR, Cramer SC. Robot-based hand motor therapy after stroke. Brain. 2007;131(2):425-37.

136. van Ommeren A, Radder B, Buurke J, Kottink A, Holmberg J, Sletta K, et al.. The Effect of Prolonged Use of a Wearable Soft-Robotic Glove Post Stroke-a Proof-of-Principle. Proceedings of the $7^{\text {th }}$ IEEE International Conference on Biomedical Robotics and Biomechatronics (Biorob), Enschede, the Netherlands, 2018 Aug 26-29; p. 445-449. 
137. Radder B, Prange-Lasonder GB, Kottink Al, Holmberg J, Sletta K, Van Dijk M, et al. The effect of a wearable soft-robotic glove on motor function and functional performance of older adults. Assistive Technology. 2018:1-7.

138. Radder B, Prange-Lasonder G, Kottink A, Gaasbeek L, Holmberg J, Meyer T, et al.. Preliminary findings of feasibility of a wearable soft-robotic glove supporting impaired hand function in daily life. Proceedings of the International conference on Information and Communication Technologies for Ageing Well and e-Health; 2016.

139. Prange-Lasonder GB, Radder B, Kottink Al, Melendez-Calderon A, Buurke JH, Rietman JS. Applying a soft-robotic glove as assistive device and training tool with games to support hand function after stroke: Preliminary results on feasibility and potential clinical impact. Proceedings of the $15^{\text {th }}$ IEEE International Conference on Rehabilitation Robotics (ICORR), Londen, United Kingdom, 2017 July 17-20 11-14; p. 1401-1406.

140. Santisteban L, Térémetz M, Bleton J-P, Baron J-C, Maier MA, Lindberg PG. Upper limb outcome measures used in stroke rehabilitation studies: a systematic literature review. PloS one. 2016;11(5):e0154792.

141. Patterson TS, Bishop M, McGuirk T, Sethi A, Richards L. Reliability of upper extremity kinematics while performing different tasks in individuals with stroke. Journal of motor behavior. 2011;43(2):121-30.

142. Collins KC, Kennedy NC, Clark A, Pomeroy VM. Getting a kinematic handle on reach-to-grasp: A meta-analysis. Physiotherapy. 2017.

143. Brooke J.SUS-A quick and dirty usability scale. Usability evaluation in industry. 1986;189(194):47.

144. Van Ommeren A, Radder B, Kottink A, Buurke J, Prange-Lasonder G, Rietman J. Quantifying Upper Extremity Performance with and Without Assistance of a Soft-robotic Glove in Elderly Patients: a Kinematic Analysis. Journal of Rehabilitation Medicine. 2019;51(4):298-306.

145. Grujic T, Bonkovic M. Measurement and analysis of human hand kinematics. International Journal of Medical, Health, Biomedical and Pharmaceutical Engineering. 2015;9:97-102.

146. Augurelle A-S, Smith AM, Lejeune T, Thonnard J-L. Importance of cutaneous feedback in maintaining a secure grip during manipulation of hand-held objects. Journal of Neurophysiology. 2003;89(2):665-71.

147. Chiavenna A, Scano A, Malosio M, Molinari Tosatti L, Molteni F. Assessing user transparency with muscle synergies during exoskeleton-assisted movements: a pilot study on the LIGHTarm device for neurorehabilitation. Applied bionics and biomechanics. 2018;2018.

148. Jarrassé N, Proietti T, Crocher V, Robertson J, Sahbani A, Morel G, et al. Robotic exoskeletons: a perspective for the rehabilitation of arm coordination in stroke patients. Frontiers in human neuroscience. 2014;8:947.

149. Proietti T, Crocher V, Roby-Brami A, Jarrasse N. Upper-limb robotic exoskeletons for neurorehabilitation: a review on control strategies. IEEE reviews in biomedical engineering. 2016;9:4-14.

150. Gates DH, Walters LS, Cowley J, Wilken JM, Resnik L. Range of motion requirements for upper-limb activities of daily living. American Journal of Occupational Therapy. 2016;70(1):7001350010p1-p10.

151. Cirstea M, Levin MF. Compensatory strategies for reaching in stroke. Brain. 2000;123(5):94053.

152. Mehrholz J, Hädrich A, Platz T, Kugler J, Pohl M. Electromechanical and robot-assisted arm training for improving generic activities of daily living, arm function, and arm muscle strength after stroke. Cochrane database of systematic reviews. 2012(6).

153. Xiloyannis M, Cappello L, Binh KD, Antuvan CW, Masia L. Preliminary design and control of a soft exosuit for assisting elbow movements and hand grasping in activities of daily living. Journal of Rehabilitation and Assistive Technologies Engineering. 2017;4:2055668316680315.

154. In H, Kang BB, Sin M, Cho K-J. Exo-glove: A wearable robot for the hand with a soft tendon routing system. IEEE Robotics \& Automation Magazine. 2015;22(1):97-105.

155. Hackel ME, Wolfe GA, Bang SM, Canfield JS. Changes in hand function in the aging adult as determined by the Jebsen Test of Hand Function. Physical Therapy. 1992;72(5):373-7. 
156. Jebsen RH, Taylor N, Trieschmann R, Trotter MJ, Howard LA. An objective and standardized test of hand function. Archives of physical medicine and rehabilitation. 1969;50(6):311-9.

157. Chen J, Nichols D, Brokaw EB, Lum PS. Home-based therapy after stroke using the hand spring operated movement enhancer (HandSOME). IEEE Transactions on Neural Systems and Rehabilitation Engineering. 2017;25(12):2305-12.

158. Wolf SL, Sahu K, Bay RC, Buchanan S, Reiss A, Linder S, et al. The HAAPI (Home Arm Assistance Progression Initiative) trial: a novel robotics delivery approach in stroke rehabilitation. Neurorehabilitation and neural repair. 2015;29(10):958-68.

159. Kwakkel G, van Peppen R, Wagenaar RC, Wood Dauphinee S, Richards C, Ashburn A, et al. Effects of augmented exercise therapy time after stroke: a meta-analysis. stroke. 2004;35(11):2529-39.

160. Strong K, Mathers C, Bonita R. Preventing stroke: saving lives around the world. The Lancet Neurology. 2007;6(2):182-7.

161. Raghavan P. The nature of hand motor impairment after stroke and its treatment. Current treatment options in cardiovascular medicine. 2007;9(3):221-8.

162. Dobkin BH. Rehabilitation after stroke. New England Journal of Medicine. 2005;352(16):167784.

163. Nilsson $M$, Ingvast J, Wikander J, von Holst $H$. The Soft Extra Muscle system for improving the grasping capability in neurological rehabilitation. Proceedings of the 2012 IEEE-EMBS Conference on Biomedical Engineering and Sciences (IECBES), Langkawi, Malaysia, 2012 Dec 17-19; p.412-417.

164. Heumer G, Amor HB, Jung B. Grasp recognition for uncalibrated data gloves: A machine learning approach. Presence: Teleoperators and Virtual Environments. 2008;17(2):121-42.

165. Castellini C, Orabona F, Metta G, Sandini G. Internal models of reaching and grasping. Advanced Robotics. 2007;21(13):1545-64.

166. Kortier HG, Sluiter VI, Roetenberg D, Veltink PH. Assessment of hand kinematics using inertial and magnetic sensors. Journal of neuroengineering and rehabilitation. 2014;11(1):70.

167. Luinge HJ, Veltink PH, Baten CT. Ambulatory measurement of arm orientation. Journal of biomechanics. 2007;40(1):78-85.

168. Carpinella I, Cattaneo D, Ferrarin M. Quantitative assessment of upper limb motor function in Multiple Sclerosis using an instrumented Action Research Arm Test. Journal of neuroengineering and rehabilitation. 2014;11(1):67.

169. Mason CR, Gomez JE, Ebner TJ. Hand synergies during reach-to-grasp. Journal of neurophysiology. 2001;86(6):2896-910.

170. Hastie T, Tibshirani R, Friedman J. Support vector machines and flexible discriminants. The elements of statistical learning: Springer; 2009. p. 417-58.

171. Hastie T, Tibshirani R, Friedman J. Overview of supervised learning. The elements of statistical learning: Springer; 2009. p. 9-41.

172. Beleites C, Neugebauer U, Bocklitz T, Krafft C, Popp J. Sample size planning for classification models. Analytica chimica acta. 2013;760:25-33.

173. Feigin VL, Norrving B, Mensah GA. Global burden of stroke. Circulation research. 2017;120(3):439-48.

174. Hatem SM, Saussez G, della Faille M, Prist V, Zhang X, Dispa D, et al. Rehabilitation of motor function after stroke: a multiple systematic review focused on techniques to stimulate upper extremity recovery. Frontiers in human neuroscience. 2016;10:442.

175. Dobkin BH. Strategies for stroke rehabilitation. The Lancet Neurology. 2004;3(9):528-36.

176. Mayo NE, Wood-Dauphinee S, Côte R, Durcan L, Carlton J. Activity, participation, and quality of life 6 months poststroke. Archives of physical medicine and rehabilitation. 2002;83(8):103542.

177. Webster JB, Darter BJ. Principles of Normal and Pathologic Gait. Atlas of Orthoses and Assistive Devices: Elsevier; 2019. p. 49-62.

178. Gandolla M, Ferrante S, Ferrigno G, Baldassini D, Molteni F, Guanziroli E, et al. Artificial neural network EMG classifier for functional hand grasp movements prediction. Journal of International Medical Research. 2017;45(6):1831-47. 
179. Leon B, Basteris A, Infarinato F, Sale P, Nijenhuis S, Prange G, et al. Grasps recognition and evaluation of stroke patients for supporting rehabilitation therapy. BioMed research international. 2014;2014.

180. Lee SW, Wilson KM, Lock BA, Kamper DG. Subject-specific myoelectric pattern classification of functional hand movements for stroke survivors. IEEE Transactions on Neural Systems and Rehabilitation Engineering. 2010;19(5):558-66.

181. de Vries J, van Ommeren A, Prange-Lasonder G, Rietman J, Veltink P. Detection of the intention to grasp during reach movements. Journal of Rehabilitation and Assistive Technologies Engineering. 2018;5:2055668317752850.

182. Collins KC, Kennedy NC, Clark A, Pomeroy VM. Kinematic components of the reach-to-target movement after stroke for focused rehabilitation interventions: systematic review and metaanalysis. Frontiers in neurology. 2018;9.

183. Zhou X, Zhang X, Wang B. Online support vector machine: a survey. Harmony Search Algorithm: Springer; 2016. p. 269-78.

184. Zucchini W, MacDonald IL, Langrock R. Hidden Markov models for time series: an introduction using R: Chapman and Hall/CRC; 2016.

185. Wissel T, Pfeiffer T, Frysch R, Knight RT, Chang EF, Hinrichs H, et al. Hidden Markov model and support vector machine based decoding of finger movements using electrocorticography. Journal of neural engineering. 2013;10(5):056020.

186. Spinhof L, Calvi L. User and task analysis in a home care environment. 20th international symposium on human factors in telecommunication, Sofia Antipolis, France; 2006 March 20-2: Citeseer 2006.

187. Nedopil C, Schauber C, Glende S. Guideline: the art and joy of user integration in AAL projects. Ambient Assisted Living Association: Brussels, Belgium. 2013.

188. Chu C-Y, Patterson RM. Soft robotic devices for hand rehabilitation and assistance: a narrative review. Journal of neuroengineering and rehabilitation. 2018;15(1):9.

189. Shahid T, Gouwanda D, Nurzaman S. Moving toward soft robotics: A decade review of the design of hand exoskeletons. Biomimetics. 2018;3(3):17.

190. Yap HK, Lim JH, Nasrallah F, Yeow C-H. Design and preliminary feasibility study of a soft robotic glove for hand function assistance in stroke survivors. Frontiers in neuroscience. 2017;11:547.

191. Leonard-Barton D. Core capabilities and core rigidities: A paradox in managing new product development. Strategic management journal. 1992;13(S1):111-25.

192. Fishbein $M$, Ajzen I. Belief, attitude, intention and behavior: An introduction to theory and research. Addison-Wesley, Reading, MA. 1975.

193. Davis FD. Perceived usefulness, perceived ease of use, and user acceptance of information technology. MIS quarterly. 1989:319-40.

194. Venkatesh V, Davis FD. A theoretical extension of the technology acceptance model: Four longitudinal field studies. Management science. 2000;46(2):186-204.

195. Ajzen I. The theory of planned behavior. Organizational behavior and human decision processes. 1991;50(2):179-211.

196. Wolf SL, Kwakkel G, Bayley M, McDonnell M, Group UESAW. Best practice for arm recovery post stroke: an international application. Physiotherapy. 2016;102(1):1-4.

197. Cabrita M, op den Akker H, Tabak M, Hermens HJ, Vollenbroek-Hutten MM. Persuasive technology to support active and healthy ageing: An exploration of past, present, and future. Journal of biomedical informatics. 2018;84:17-30.

198. Conner TS, Mehl MR. Handbook of research methods for studying daily life. Guilford Press, New York, United States. 2011.

199. Singer JD, Willett JB, Willett JB. Applied longitudinal data analysis: Modeling change and event occurrence: Oxford university press. 2003.

200. Radder B, Prange-Lasonder GB, Kottink Al, Melendez-Calderon A, Buurke JH, Rietman JS. Feasibility of a wearable soft-robotic glove to support impaired hand function in stroke patients. Journal of rehabilitation medicine. 2018;50(7):598-606.

201. Kintsch A, DePaula R. A framework for the adoption of assistive technology. SWAAAC 2002: 
Supporting learning through assistive technology. 2002:1-10.

202. Holmes D, Charles D, Morrow P, McClean S, McDonough S. Usability and performance of Leap Motion and Oculus Rift for upper arm virtual reality stroke rehabilitation. Proceedings of the 11th International Conference on Disability, Virtual Reality \& Associated Technologies (ICDVRAT). Los Angeles, United States, 2016 Sep 20-22.

203. Hu X, Tong KY, Song R, Tsang VS, Leung PO, Li L. Variation of muscle coactivation patterns in chronic stroke during robot-assisted elbow training. Archives of physical medicine and rehabilitation. 2007;88(8):1022-9.

204. Kang YJ, Ku J, Han K, Kim SI, Yu TW, Lee JH, et al. Development and clinical trial of virtual reality-based cognitive assessment in people with stroke: preliminary study. CyberPsychology \& Behavior. 2008;11(3):329-39.

205. Jie S, Haoyong Y, Chaw TL, Chiang CC, Vijayavenkataraman S. An Interactive Upper Limb Rehab Device for Elderly Stroke Patients. Procedia CIRP. 2017;60:488-93.

206. Pohl PS, Kemper S, Siengsukon CF, Boyd L, Vidoni ED, Herman RE. Dual-task demands of hand movements for adults with stroke: a pilot study. Topics in stroke rehabilitation. 2011;18(3):238-47.

207. Yang Y-R, Chen Y-C, Lee C-S, Cheng S-J, Wang R-Y. Dual-task-related gait changes in individuals with stroke. Gait \& posture. 2007;25(2):185-90.

208. Yang $Y-R$, Wang $R-Y, C$ en $Y-C$, Kao M-J. Dual-task exercise improves walking ability in chronic stroke: a randomized controlled trial. Archives of physical medicine and rehabilitation. 2007;88(10):1236-40.

209. Choi YM, Sprigle SH. Approaches for evaluating the usability of assistive technology product prototypes. Assistive Technology ${ }^{\circledR}$. 2011;23(1):36-41.

210. Ambrosini E, Ferrante S, Rossini M, Molteni F, Gföhler M, Reichenfelser W, et al. Functional and usability assessment of a robotic exoskeleton arm to support activities of daily life. Robotica. 2014;32(8):1213-24.

211. Nijenhuis SM, Prange-Lasonder GB, Stienen AH, Rietman JS, Buurke JH. Effects of training with a passive hand orthosis and games at home in chronic stroke: a pilot randomised controlled trial. Clinical rehabilitation. 2017;31(2):207-16.

212. Nijenhuis SM, Prange-Lasonder GB, Amirabdollahian F, Infarinato F, Buurke JH, Rietman JS. Feasibility of a second iteration wrist and hand supported training system for selfadministered training at home in chronic stroke. ROLL UP YOUR SLEEVES! 2018:131.

213. McAuley E, Duncan T, Tammen VV. Psychometric properties of the Intrinsic Motivation Inventory in a competitive sport setting: A confirmatory factor analysis. Research quarterly for exercise and sport. 1989;60(1):48-58.

214. van Gemert-Pijnen JE, Nijland N, van Limburg M, Ossebaard HC, Kelders SM, Eysenbach G, et al. A holistic framework to improve the uptake and impact of eHealth technologies. Journal of medical Internet research. 2011;13(4).

215. Krabbe D, Nijland M. Novalab: de brug tussen wetenschap en praktijk. CVA kennis netwerk Nederland - Mee(r) doen na een CVA. Zeist, the Netherlands 2015. 



\section{Summary}

Not only are our hands essential to perform daily life activities, they are often used to express respect, love, and anger via non-verbal communication as well. After stroke, many patients have impaired hand and arm function. The use of rehabilitation robotics has shown to be useful to overcome such impairments. However, evidence of a transfer of robotic training effects towards improved functionality in daily life remains limited. After the rehabilitation period, limitations in performance of daily activities affects the independence and quality of life of stroke patients. Stroke patients can increase functional independence if they are supported directly by technology during activities of daily living ( $A D L)$ in their homes. However, in practice it appears to be difficult to apply such smart technology in ADL of stroke survivors possibly because the needs and preferences of the user are not adequately reflected in the devices. The ironHand glove, a wearable soft-robotic glove, was developed according to a user-centered design in order to support grip strength during daily life activities of people experiencing hand function problems. This thesis aims to increase our understanding on user requirements of assistive technology (AT), and on the usability and effectiveness of the ironHand system, to contribute to the development of assistive technologies to support the hand of stroke patients during ADL at home that meet the needs and preferences of the end-user. To reach this goal, the following research questions have been proposed in chapter 1 :

1. What defines the user preferences concerning assistive technology designed for the upper limb of stroke patients?

2. Does a wearable soft-robotic glove improve task performance directly?

3. Does prolonged, unsupervised, use of a wearable soft-robotic glove during ADL at home improve task performance of stroke patients?

4. Considering the limitations of pressure sensing as control input for the device, could the control of the grip-supporting glove be improved?

\section{User preferences and user experience}

We started with a systematic review in chapter 2, to get more insight into the factors that can bring the design of AT to higher levels of satisfaction and acceptance. User perspectives from focus groups, questionnaires and interviews of nine studies were gathered. Barriers and enablers influencing the adoption of AT for the upper limb after stroke emerged within 5 overarching, but highly interdependent themes: (1) promoting hand and arm performance; (2) attitude toward technology; (3) decision process; (4) usability; and (5) practical applicability. The interdependency of the identified themes implies that all aspects influencing user perspectives of AT need to be considered when developing AT, and not just few of them, to enhance its chance of acceptance. 
In chapter 3 user requirements of AT for the upper limb were identified through a focus group. The focus group results showed that unobtrusive support, intuitive use, and adaptability to the individual user and his or her disease severity are key for stroke patients. Opinions with respect to specific preferred control- and support options were diverse. After use, user preferences of four different modalities to control the soft-robotic glove, namely force, muscle activation, voice and wink, were gathered in chapter 4. User perspectives greatly varied, with four participants favoring force control, while each of the other modalities were chosen as favorite by two participants. System Usability Scale (SUS) scores were, on average, 70 or higher for force and voice control while muscle activation and wink received scores between 50-70. Usability of the ironHand glove, activated by pressure sensors, was assessed after one-time use (chapter 6) and prolonged use (chapter 7). In both studies, promising scores on the SUS were found (mean 71.6 (chapter 6), median 87.5 (chapter 7)). The SUS was supplemented with a semi-structured interview in chapter $\mathbf{6}$. In general, participants were positive about the glove. Several participants appreciated the glove having two glove-free fingers, which helped to maintain sensation during object manipulation. Also, the experience that the glove actually supports grip strength increased their perceived grip assurance and confidence when moving, which was appreciated. Contrary, the limitation of movement, the glove being not water resistant, and the loss of sensation were mentioned as points for improvement.

\section{Direct effect of the soft-robotic glove}

Feasibility of each of four control modalities (force, muscle activity, voice and wink) was tested to supplement subjective information in chapter 4 . The number of attempts needed to control the glove did not significantly differ, and although highly interdependent, time needed to perform a reach-and-grasp task was significantly lowest with force control. In general, participants were positive about the control modalities, however, a weak correlation of subjective outcomes and objective measures was found.

We aimed to assess the direct effect of the ironHand glove on functional performance of the hand and arm in older adults (chapter 5) and the stroke population (chapter $6 \&$ 7). Maximal pinch strength was positively influenced by the ironHand glove (chapter 6). In chapter 7, the direct effect of the glove was assessed with the Jebsen-Taylor Hand Function Test (JTHFT) in stroke. In line with previously performed studies assessing the effect of the ironHand glove on performance of the JTHFT in elderly, we did not find a positive effect of the glove on JTHFT performance. To better understand its influence on movement, in chapter 5 and chapter 6 kinematics of the upper limb were assessed during a reachand-grasp task in which a cylindrical object had to be placed onto a platform at shoulder height with and without using the glove. In older adults with self-reported hand function 
limitations, the glove did not improve total movement time. When looking at kinematics in more detail, relative time needed to transport the heavy object was shorter with glove, while relative time needed to grasp the heavy object was longer. Additionally, transporting light objects occurred with a lower peak velocity and larger elbow extension, and grasping of the object involved a larger hand opening as compared to without glove. In stroke, alterations in joint excursion were particularly found in the wrist and shoulder, with corresponding larger compensatory movements of the trunk. In extension to the study with older adults, participants also wore the glove without being activated as an additional condition to assess the transparency of the glove. Activating the glove itself did not affect movement performance negatively, as compared to the glove on - support off. In contrast, negative effects on movement execution with activated glove use were related to wearing the glove itself.

\section{Effect of prolonged use of the soft-robotic glove at home}

Five stroke patients successfully used the ironHand glove four weeks at home. The effect of prolonged home-use the glove on JTHFT performance was assessed in chapter 7. Before and after home-use, the JTHFT was tested in a lab-environment. The prolonged use of the glove resulted in improved supported, and even unsupported, functional performance during tasks related to activities of daily life, as measured with the JTHFT.

\section{Control of the soft-robotic glove}

Based on the findings of the preceding chapters that focused on the user requirement identification, usability and effectiveness of the ironHand system, the idea arose to research the possibility to detect the intention to grasp earlier than the pressure sensors of the current version of the ironHand system in both healthy subjects (chapter 8) and stroke patients (chapter 9). In both studies, promising user-dependent accuracies were found $(98.2 \%$ in healthy and $96.8 \%$ in stroke). In a user-independent situation, accuracies of $91.4 \%$ and $83.3 \%$ were achieved in healthy subjects and stroke patients respectively. In the offline setting, grasp could be classified accurately in more than $90 \%$ when using only half of the movement length in stroke. This would allow for an earlier detection of grasp of 750ms.

\section{Discussion and conclusion}

The main findings of the studies are discussed in conjunction in chapter 10, giving rise to recommendations for future research and development. In general, AT should be designed according to a holistic approach, taking the interdependencies of factors related to the adoption 
of AT into account. This thesis also indicated a clear need for personalization of devices, sufficient familiarization time with AT during research, and the need for suitable outcome measures when assessing their effects. Provided that the abovementioned recommendations are considered adequately, this thesis demonstrated the potential of the ironHand glove to be used both as assistive and therapeutic device, increasing hand strength directly and improving unsupported hand function after only four week use during ADL. 



\section{Samenvatting}

Onze handen zijn niet alleen onmisbaar voor het uitvoeren van dagelijkse activiteiten, we gebruiken ze ook om respect, liefde en woede uit te drukken via non-verbale communicatie. $\mathrm{Na}$ een beroerte hebben veel patiënten een verminderde hand- en armfunctie. De inzet van revalidatierobotica kan het mogelijk maken om het herstel van hand- en armfunctie te stimuleren. Er is echter beperkt bewijs dat effecten van robottrainingen vertaalbaar zijn naar functionaliteit in het dagelijks leven. Beperkingen tijdens dagelijkste activiteiten, die na de revalidatie periode worden ervaren, hebben een negatieve invloed op de onafhankelijk van patiënten en beïnvloeden daarmee de kwaliteit van leven. Patiënten zouden baat kunnen hebben van technologie wanneer ze in hun thuissituatie ondersteund kunnen worden tijdens dagelijkse activiteiten. In de praktijk blijkt het echter moeilijk te zijn om dergelijke robotica toe te passen in algemeen dagelijkse levensverrichtinge (ADL), mogelijk omdat de behoeften en voorkeuren van de gebruiker niet worden weerspiegeld in de technologie. De ironHand, een draagbare zacht-robotische handschoen, is ontwikkeld volgens een gebruikersgericht ontwerp om de grijpkracht te ondersteunen tijdens de dagelijkse activiteiten van mensen met handfunctie beperkingen. In dit proefschrift willen we meer inzicht krijgen in het effect van het huidige ontwerp van de ironHand op de taakuitvoering en op de factoren, in termen van gebruikersperspectieven en het ontwerp, die kunnen bijdragen aan de verbetering van de ontwikkeling van ondersteunende technologie (OT) ter ondersteuning van de hand van beroerte patiënten tijdens dagelijkse activiteiten. Om dit doel te bereiken zijn in hoofdstuk 1 de volgende onderzoeksvragen voorgesteld:

1. Wat bepaalt de voorkeur van de gebruiker met betrekking tot OT voor de hand en/of arm voor patiënten na een beroerte?

2. Verbetert een draagbare zacht-robotische handschoen de uitvoering van bewegingen direct?

3. Is het uitvoeren van taken door CVA patiënten beter na langdurig thuisgebruik van een zacht-robotische handschoen tijdens dagelijkse activiteiten?

4. Wat kan, gezien de beperkingen van druksensoren als controlemechanisme voor het apparaat, de controle van de handschoen die de greep ondersteunt, verbeteren?

\section{Gebruikersvoorkeuren en gebruikerservaring}

We zijn begonnen met een systematische review in hoofdstuk 2, om meer inzicht te krijgen in de factoren die het ontwerp van OT naar een hoger niveau van gebruikerstevredenheid en acceptatie kunnen brengen. Gebruikersperspectieven van negen studies zijn verzameld waarbij 
focusgroepen, vragenlijsten en interviews zijn gebruikt. Barrières en faciliterende factoren die de adoptie van OT voor de bovenste ledematen na een beroerte beïnvloeden, zijn onderverdeeld in 5 overkoepelende, maar sterk van elkaar afhankelijke, thema's: (1) het bevorderen van hand- en armprestaties; (2) houding ten opzichte van technologie; (3) besluitvormingsproces; (4) bruikbaarheid; en (5) praktische toepasbaarheid. De onderlinge afhankelijkheid van de geïdentificeerde thema's impliceert dat bij de ontwikkeling van OT rekening moet worden gehouden met alle aspecten die van invloed zijn op de gebruikersperspectieven van OT om de kans op acceptatie ervan te vergroten.

In hoofdstuk 3 zijn de gebruikerseisen van OT voor de hand en arm geïdentificeerd met een focusgroep. De resultaten van de focusgroep toonden aan dat onopvallende en nietbelemmerende ondersteuning, intuïtief gebruik en aanpasbaarheid aan de individuele gebruiker en de ernst van zijn of haar ziekte cruciaal zijn voor patiënten na een beroerte. $\mathrm{Na}$ gebruik van de zacht-robotische handschoen werden de gebruikersvoorkeuren verzameld van vier verschillende modaliteiten om de handschoen aan te sturen, namelijk kracht, spieractivatie, stem en knipoog, in hoofdstuk 4. De perspectieven van de gebruiker liepen sterk uiteen, waarbij vier deelnemers de voorkeur gaven aan aansturing door krachtsensoren, terwijl alle andere modaliteiten door twee deelnemers als favoriet werden gekozen. System Usability Scale (SUS) scores waren gemiddeld 70 of hoger voor kracht- en stemaansturing, terwijl de scores voor spieractivatie en knipoog tussen de 50-70 lagen. De bruikbaarheid van de ironHand handschoen, aangestuurd door krachtsensoren, werd ook gescoord na eenmalig gebruik (hoofdstuk 6) en langdurig gebruik (hoofdstuk 7). In beide studies werden veelbelovende scores op de SUS gerapporteerd. In hoofdstuk 6 is de informatie van de SUS aangevuld met een semigestructureerd interview. Daaruit bleek dat de deelnemers over het algemeen positief zijn over de handschoen. Verschillende deelnemers waardeerden dat de handschoen twee handschoenvrije vingers heeft, wat hielp om het gevoel te behouden tijdens het manipuleren van objecten. Ook de ervaring dat de handschoen de grijpkracht daadwerkelijk ondersteunt, waardoor zelfvertrouwen bij het bewegen vergroot werd, werd op prijs gesteld. Daarentegen werden de beperking van de bewegingsvrijheid, het feit dat de handschoen niet waterbestendig is en het verlies van sensatie genoemd als punten voor verbetering.

\section{Het directe effect van de ironHand handschoen}

De toepasbaarheid van elk van de vier aansturingsmodaliteiten (kracht, spieractivatie, spraak en knipoog) is getest om de subjectieve informatie in hoofdstuk 4 aan te vullen. Het aantal pogingen dat nodig was om de handschoen aan te sturen verschilde niet significant tussen de verschillende modaliteiten, en hoewel sterk van elkaar afhankelijk, was de tijd die nodig was 
om een reik- en grijptaak uit te voeren het laagst wanneer de handschoen weer aangestuurd door de krachtsensoren.

Het directe effect van de ironHand handschoen op de functionele prestaties van de hand en arm hebben we onderzocht bij ouderen (hoofdstuk 5) en bij mensen die een beroerte hebben gehad (hoofdstuk 6). In beide studies werd de kinematica van de hand- en arm gemeten tijdens een reik- en grijptaak waarbij een cilindrisch voorwerp op een platform op schouderhoogte moest worden geplaatst. Deze taak werd zowel met als zonder handschoen uitgevoerd, en met een lichte en zware cilinder. Bij ouderen met zelf-gerapporteerde functiebeperkingen van de hand werd geen positieve invloed van de handschoen op de totale bewegingstijd gevonden. Bij de uitvoering met handschoen was de relatieve tijd die nodig was om het zware object te vervoeren korter, terwijl de relatieve tijd die nodig was om het zware object vast te pakken langer was. Bovendien vond het verplaatsen van de lichte cilinder plaats met een lagere snelheid van de hand en een grotere ellebooghoek, en lieten mensen een grotere handopening zien bij het vastpakken van de cilinder dan wanneer de taak werd uitgevoerd zonder handschoen. Bij patiënten na een beroerte werden vooral veranderingen in de gewrichtsbeweging van pols en schouder gevonden, waarbij evenredig grote compensatiebewegingen van de romp te zien waren. In tegenstelling tot de studie bij ouderen hebben we in de studie bij mensen die een beroerte hebben gehad ook een derde conditie opgenomen waarbij de deelnemers de handschoen droegen zonder dat deze geactiveerd was. Er werden geen negatieve invloeden van de geactiveerde handschoen zelf gevonden op de bewegingsprestatie in vergelijking met de niet-geactiveerde handschoen. De gevonden negatieve effecten worden dus waarschijnlijk veroorzaakt door het dragen van een handschoen op zichzelf. In hoofdstuk 7 werd het directe effect van de handschoen gemeten met behulp van de Jebsen-Taylor Hand Function Test (JTHFT). Wederom werd geen positief direct effect van de handschoen op de bewegingstijd gevonden. Daarentegen werd de maximale knijpsterkte wel positief beïnvloed door de handschoen (hoofdstuk 6).

\section{Het effect van langdurig thuisgebruik van de ironHand handschoen}

De handschoen is met succes vier weken thuisgebruik door vijf beroerte patiënten. Het effect van langdurig thuisgebruik van de handschoen op de prestatie van JTHFT werd beoordeeld in hoofdstuk 7. Zowel voor als na thuisgebruik werd de JTHFT getest in een lab-omgeving. Het langdurig gebruik van de handschoen resulteerde in een verbeterde ondersteunde (met handschoen) en niet-ondersteunde (zonder handschoen) functionele prestatie tijdens taken die verband houden met activiteiten in het dagelijks leven, zoals in deze studie gemeten met de JTHFT. 


\section{Aansturing van de ironHand handschoen}

Aangezien (delen van) taken trager worden uitgevoerd met de handschoen, wat te wijten kan zijn aan het verminderde gevoel door het dragen van de handschoen, werd onderzocht of het mogelijk is om de intentie tot grijpen eerder te detecteren dan de krachtsensoren kunnen. Dit is onderzocht bij zowel gezonde proefpersonen (hoofdstuk 8 ) als bij mensen die een beroerte hebben gehad (hoofdstuk 9). In beide studies werden veelbelovende nauwkeurigheden gevonden wanneer het model getraind was op dezelfde persoon $(98.2 \%$ bij gezonde personen en $96.8 \%$ bij beroerte). In een gebruikersonafhankelijke situatie werden gemiddelde nauwkeurigheden van $91.4 \%$ en $83.3 \%$ gevonden bij respectievelijk gezonde proefpersonen en patiënten na een beroerte. In de offline setting van onze studies kon de intentie tot grijpen van patiënten na een beroerte in meer dan $90 \%$ van de voorspellingen nauwkeurig worden gedetecteerd wanneer slechts de helft van de bewegingslengte werd gebruikt. Dit zou een eerdere detectie van grijpen van 750 ms mogelijk maken ten opzichte van de krachtsensoren.

\section{Discussie en conclusie}

De belangrijkste bevindingen van de studies zijn gecombineerd en besproken in hoofdstuk 10, waardoor aanbevelingen voor toekomstig onderzoek en ontwikkeling opgesteld konden worden. In het algemeen moeten OT worden ontworpen volgens een holistische benadering, waarbij rekening wordt gehouden met de onderlinge afhankelijkheid van factoren die verband houden met de toepassing van OT. Dit proefschrift wees ook op een duidelijke behoefte aan personalisatie van apparaten, voldoende tijd om vertrouwd te raken met een OT, en de behoefte aan geschikte subjectieve en objectieve uitkomstmaten. Studies in dit proefschrift hebben de potentie van de ironHand handschoen laten zien om zowel als ondersteunend hulpmiddel als therapeutisch hulpmiddel te kunnen gebruiken, waardoor de kracht van de hand direct wordt verhoogd en de niet-ondersteunde handfunctie wordt verbeterd na slechts 4 weken gebruik tijdens $A D L$, op voorwaarde dat de bovengenoemde aspecten voldoende worden meegenomen in het verdere ontwerp. 



\section{Dankwoord}

Een dag voordat het proefschrift naar de drukker gaat. Ja, ik ben één van de mensen die het schrijven van het dankwoord uitstelt totdat alles klaar is. Best gek, want het is juist leuk om stil te mogen staan bij de mensen die het mogelijk gemaakt hebben om dit deel van het proefschrift te kunnen schrijven. Onvoorstelbaar hoeveel mensen een onmisbare bijdrage hebben geleverd, inhoudelijk of juist vooral niet, heel erg bedankt!

Aan het einde van mijn afstudeeropdracht bij RRD zei Jaap tegen mij: "Ik zou het leuk vinden als je eens met Gerdienke gaat praten..." Best spannend want zo vaak hadden we elkaar nog niet gesproken. Gerdienke, wat hebben we ons in die vier jaar na ons gesprek vaak verbaasd over de gebeurtenissen en ontwikkelingen binnen het eNHANCE project. Van faillissement tot inbraak, het is te veel om op te noemen. Wat fijn dat jij, op je zwangerschapsverloffen na, een vast gegeven bent geweest. Zonder jouw nuchterheid, steun en positiviteit zou dit proefschrift er niet zo staan zoals het nu staat, en had ik mij de afgelopen jaren niet zo kunnen ontwikkelen. "Ik heb veel geleerd" was vaak een manier om mezelf moed in te praten, maar niet minder waar wanneer ik het heb over jouw manier van verwoorden. Meer dan eens heb je mij het zetje gegeven om te verwoorden wat ik nou precies bedoelde. Jouw deur staat altijd open, niet alleen voor inhoudelijke zaken maar ook voor alle andere onderwerpen. Bedankt!

Hans, vaak begonnen we onze overleggen over de miserie en diepe dalen binnen het eNHANCE project. De eerste drie jaar was ieder overleg een pijnlijk reflectiemoment; kijken we nou naar plan B, C, of D?? Gelukkig had jij meer vertrouwen dat mijn promotie wel goed zou komen en kreeg je het elke keer voor elkaar om mij een stukje meer gemotiveerd en vastberaden het overleg uit te laten gaan. Ik heb genoten van jouw enthousiasme en hoe jij het voor elkaar krijgt altijd de behoefte van de patiënt centraal te houden. Zelfs tijdens inhoudelijke discussies samen met Peter. Wat heb ik geluk gehad om jullie als combinatie van klinisch en technisch achter me te hebben staan en te sturen. Peter, ik heb veel mogen leren van jouw technische blik en kennis. Ik vind het knap dat elke zin die je uitspreekt of schrijft de lading dekt. Bedankt voor alle feedback en gesprekken.

Jaap, ook al sta jij niet formeel in het rijtje van promotoren, ook jij hebt een groot aandeel gehad in dit resultaat. Naast onze inspirerende inhoudelijke overleggen wil ikje ook ontzettend bedanken voor het geven van allerlei kansen en leuke uitstapjes buiten het project. Leren 'nee' zeggen is misschien wel één van mijn grootste uitdagingen geweest. Alhoewel, vakantie opnemen was ook elk jaar een vast punt op het functioneringsgesprek... 
Ik heb mij vaak verbaasd over de wendingen van het eNHANCE project, maar ik heb mij nog meer verbaasd (wel positief dit keer) over de motivatie en belangeloosheid van deelnemers van alle onderzoeken. Geen enkel experiment in mijn proefschrift heeft een directe bijdrage kunnen leveren aan de handfunctie van de deelnemers. Toch stonden de deelnemers elke keer weer klaar, vaak voor lange en uitputtende experimenten, hopend dat ze een bijdrage kunnen leveren aan iets dat uiteindelijk andere CVA patiënten kan helpen. Ontzettend bedankt. Ook ontzettend bedankt voor gezellige gesprekken, met vaak heftige verhalen. Naast jullie onmisbare bijdrage in het verzamelen van data, hebben de gesprekken mij keer op keer geholpen om alles te relativeren.

I would like to thank the members of my graduation committee for the willingness to read and evaluate my thesis and be part of my PhD defense.

Marcel, Martijn en Laura, wat fijn dat jullie medestanders waren binnen het project. Bedankt voor alle gezellige uren in het lab. Martin, Mario and Jackie, I would like to thank you as well for the nice collaboration.

Natuurlijk wil ik ook even stilstaan bij alle collega's van RRD. Wat voel je je snel thuis bij RRD, bedankt daarvoor. In het bijzonder dank aan Inger, Gerda, Hester, Wies en Sandra; jullie hebben een gave om tijd te vinden in agenda's waarin al 3 of 4 dubbele afspraken staan. Inger en Brigitte, bedankt voor jullie oprechte interesse in alles en iedereen.

Leendert?! Waarschijnlijk hoor je al precies hoe ik het zou zeggen als ik wéér eens je kamer binnen kwam. De ontelbare keren dat je weer eens ad hoc een creatieve oplossing moest bedenken voor een lab opstelling of een analyse, of dat je de tijd nam om mijn gezucht en gesteun aan te horen. Het feit dat ik wist dat jij altijd achter me stond om mee te denken heb ik ontzettend gewaardeerd, bedankt.

Jos - wat hebben we verrassend leuke gesprekken gehad over allerlei zaken zoals data veiligheid. Ik ben heel blij en dankbaar dat jij het design voor de voorkant van dit proefschrift wilde maken.

Erik - ook jij verdient hier een plek omdat ik zonder jouw ontcijfer-kunsten de helft van de feedback van Hans niet had kunnen lezen...

Sharon - Ook al hebben we maar 1 jaar samengewerkt bij RRD ben ik ontzettend blij dat we nog contact hebben. Ik heb veel gehad aan je nuchterheid en bedankt voor alle tips en weetjes.

Kamergenoten - Josien, Sanne, Wander, Stefan, Wiebe, Erik, Marijke en Fanny! Ik kon mij geen beter gezelschap (en lekkere snoepjes) wensen. Ook al was een kamer met 6 personen minder 
goed voor iedereens productiviteit, ik had het niet willen missen. Later een kamer met Fanny alleen, niet veel beter voor mijn productiviteit moet ik zeggen. Naaktslak. The first Dutch word you learned at RRD. This probably indicates the level of our conversations at the time. I couldn't imagine having a better roommate than you. I have countless memories of smelly cheeses, your slicer, and the best conversations. My level of French curse words has gone up tremendously.

Marijke - Ook al zijn we maar kort kamergenoten geweest, vind ik het bewonderenswaardig hoe vastberaden en gestructureerd je bent.

Wat was het een genoegen om te mogen samenwerken met studenten met verschillende achtergronden. Liesbeth, Tineke, Nynke, Anne, Ben en Thijs, bedankt dat jullie een bijdrage hebben willen leveren aan dit proefschrift.

Laura, Tineke, Bob, Carmen en Ben, dankzij jullie bevat dit proefschrift uiteenlopende artikelen. Ik ben blij dat we samen aan artikelen hebben kunnen schrijven.

Een speciaal woord van dank aan het samen eten eten eten groepje. Sanne, Josien, Mirka, Jan-Willem, Wander, Fanny en Stefan. We zijn allemaal een andere kant op gegaan na onze tijd bij RRD, ik ben blij om te zien dat iedereen op zijn/haar plek zit. Bedankt voor jullie oneindige interesse en gezelligheid. Stefan - Op de een of andere manier heb je vanaf het begin onvoorwaardelijk vertrouwen gehad dat ik het allemaal wel ging redden. Ook al is dat behoorlijk overdreven en nergens op gebaseerd, moet ik bekennen dat het op sommige momenten fijn is te horen: je kan het, ik geloof in je. Sanne - Je zei het vaak tegen mij: "Let it go", in de laatste fase van het schrijven van dit proefschrift heb ik hier vaak aan gedacht. Bedankt voor alle schrijfweekendjes in Lent. Geheel toevallig dat deze samenvielen met Nijmeegse vierdaagse (feesten). Ik ben trots op je, hoe je je als mens de afgelopen jaren hebt ontwikkeld, en hoe je nu in het leven staat.

Jochem - We zijn elkaar tegengekomen in een compleet andere setting, maar onze promotietrajecten liepen in fase. Jouw positieve blik op onderzoek heeft mij tot veel inzichten gebracht, bedankt hiervoor.

Wiepke - Jou wil ik bedanken voor de ontspanning, alle avonturen met de paarden, en de gezelligheid tijdens de zaterdagochtenden. Het waren mooie momenten om het onderzoek eens in alle rust te reflecteren. Het heeft mij zowel als persoon als mijn onderzoek verder gebracht.

Mijn collega's van Ecare hebben het laatste stukje van dit traject meegemaakt. Ook al heeft 
mijn huidige werk niets te maken met onderzoek en een promotietraject; jullie zijn altijd betrokken en geïnteresseerd in mij en de status van mijn proefschrift. De product specialisten en team noord, ontzettend bedankt!

En dan, mijn paranimfen Jule en Fanny. Jule, we hebben elkaar leren kennen op het meest hectische moment van mijn promotietraject. Ik vind het onvoorstelbaar hoe kalm jij altijd bent, en hoe je dit (meestal) ook op mij weet over te brengen. Fanny, it feels right to mention you twice. Your enthusiasm and support are the best. Ik ben ontzettend blij dat jullie naast mij staan op 31 oktober.

Pap, mam, bro en sis, ik hoop dat ik jullie niet via dit boekje hoef te vertellen hoe dankbaar ik ben. Mam, jij hebt er al die jaren voor gezorgd dat ik goed voor mezelf ben blijven zorgen. Je hebt geen idee hoe belangrijk het is dat jij vertrouwen in mij hebt. Pap, het was fijn om het regelmatig samen te hebben over het doen van promotieonderzoek. Jij snapt als geen ander welk traject ik heb doorgemaakt. Je snapt alleen niet waar ik mij zo druk over maak altijd. Vaak een terecht punt, maar het is makkelijker gezegd dan gedaan...

Lajla en Edgar, ik heb mij vanaf het begin thuis gevoeld in de familie Jip. Bedankt voor de gezelligheid en jullie oprechte interesse.

Bas, jouw onvoorwaardelijke steun en knuffels zijn van onschatbare waarde. Zonder jouw positiviteit had ik dit niet voor elkaar gekregen. Het was fijn om samen in hetzelfde schuitje te zitten. Jij je master naast je werk, ik mijn promotieonderzoek. Ik ben trots op je dat jij je master hebt afgerond! Na het harde werken hebben we vanaf nu eindelijk samen weekend zonder te denken 'maar eigenlijk moeten we nog...', het is klaar! 



\section{Author contributions}

\section{Journal articles}

de Vries JC, van Ommeren AL., Prange-Lasonder GB., Rietman JS., and Veltink PH (2018). Detection of the intention to grasp during reach movements. Journal of Rehabilitation and Assistive Technologies Engineering, 5, 2055668317752850.

van Ommeren AL., Smulders LC, Prange-Lasonder GB, Buurke JH., Veltink PH., and Rietman JS (2018). Assistive Technology for the Upper Extremities After Stroke: Systematic Review of Users' Needs. JMIR rehabilitation and assistive technologies, 5(2).

van Ommeren AL, Radder B, Kottink AIR, Buurke JH, Prange-Lasonder GB., and Rietman JS (2019). Quantifying Upper Extremity Performance With and Without Assistance of a SoftRobotic Glove in the Elderly Population: a Kinematic Analysis. Journal of Rehabilitation Medicine, 51(4), 298-306.

van Ommeren AL, Sawaryn B, Prange-Lasonder GB, Buurke JH, Rietman JS., and Veltink PH (2019). Detection of the intention to grasp during reaching in stroke using inertial sensing. Accepted for publication in Transactions on Neural Systems \& Rehabilitation Engineering.

\section{Conference papers}

van Ommeren AL, Prange-Lasonder GB, Rietman JS, Veltink PH., and Buurke JH (2017). Preliminary extraction of themes from a review about user perspectives on assistive technology for the upper limb after stroke. In Converging Clinical and Engineering Research on Neurorehabilitation II (pp. 323-327). Springer, Cham.

van Ommeren AL, lodice M, Main M, Pitchforth JM, Muntoni FM, Prange-Lasonder GB, Rietman JS, and Buurke JH (2018). Identification of User Requirements for Assistive Technology for Support of Upper Limb Daily Life Interactions from Stroke and Duchenne Muscular Dystrophy Patients. The Tenth International Conference on eHealth, Telemedicine, and Social Medicine, 4,45 .

van Ommeren AL, Radder B, Buurke JH, Kottink AIR, Holmberg J, Sletta K, Prange-Lasonder GB, and Rietman JS (2018). The Effect of Prolonged Use of a Wearable Soft-Robotic Glove Post Stroke-a Proof-of-Principle. In 2018 7th IEEE International Conference on Biomedical Robotics and Biomechatronics (Biorob) (pp. 445-449). 


\section{Conference contributions}

van Ommeren AL, Prange-Lasonder GB, Buurke JH, Veltink PH, Faisal AA, Bergsma A, and Rietman JS. eNHANCE - intention based enhancement of reaching and grasping during daily life tasks in the homes of stroke patients. Oral presentation at the Vereniging voor Bewegingswetenschappen Nederland PhD Day, 24 september 2015, Enschede, the Netherlands, oral presentation.

van Ommeren AL, Prange-Lasonder GB, Rietman JS, Veltink PH, and Buurke JH. Preliminary extraction of themes from a review about user perspectives on assistive technology for the upper limb after stroke. Oral presentation at the International Conference on NeuroRehabilitation, 18-21 October 2016, Segovia, Spain.

van Ommeren AL, Prange-Lasonder GB, Buurke JH, Veltink PH, and Rietman JS. Preliminary results of the direct effect of a soft-robotic glove as assistive device on movement execution in stroke. Poster presentation at the Dutch Congress on Rehabilitation Medicine, 9-10 November 2017, Maastricht, the Netherlands.

van Ommeren AL, lodice M, Main M, Pitchforth JM, Muntoni FM, Prange-Lasonder GB, Rietman JS, and Buurke JH. Identification of User Requirements for Assistive Technology for Support of Upper Limb Daily Life Interactions from Stroke and Duchenne Muscular Dystrophy Patients. Oral presentation at eTelemed, 25-29 March 2018, Rome, Italy.

van Ommeren AL, Radder B, Buurke JH, Kottink AIR, Holmberg J, Sletta K, Prange-Lasonder GB, and Rietman The Effect of Prolonged Use of a Wearable Soft-Robotic Glove Post Stroke-a Proof-of-Principle. Poster presentation at IEEE Biorob, 26-29 August 2018, Enschede, the Netherlands. 



\section{Progress range}

The following publications have been published in the Progress range by Roessingh Research and Development, Enschede, the Netherlands. Copies can be ordered, when available, via info@rrd.nl.

1. Pot JWGA, Boer $H$, van Harten WH, Hermens HJ, Seyde IER. Comprehensive NeedAssessment. Ontwikkeling van een meetinstrument voor zorgbehoeften en kwaliteitsbeoordeling door patiënten. September 1994, ISBN 90-25452-01-22

2. van Leerdam NGA, Hermens HJ. Revalidatietechnologie in Euregio. July 1995, ISBN 9075452-02-0

3. Duda L, van Noort LO, Röseler S, Greitemann BOL, van Harten WH, Klazinga Ns. Rehabilitation in Germany and the Netherlands, A comparison of two rehabilitation systems. August 1995, ISBN 90-75452-03-9

4. Hermens HJ, Nene AV, Zilvold G. Electrophysiological Kinesiology, Precossedings of the 11th congress of the International Society of Electrophysiology and Kinesiology in Enschede, the Netherlands 1996. October 1996, ISBN 90-75452-04-7

5. van Harten WH. Bouwen aan een kwaliteitssysteem in de revalidatiezorg. Een poging tot constructieve technology assessment van een kwaliteitssysteem in een gezondheidszorginstelling. December 1997, ISBN 90-75452-07-1

6. Baardman G, ljzerman MJ. Design and evaluation of a hybrid orthosis for people with paraplegia. November 1997, ISBN 90-75452-8-X

7. Hutten MMR. Lumbar Dynamometry: A useful method for assessment of patients with chronic low back pain? November 1999, ISBN 90-75452-13-6

8. van der Salm A, van Harten WH, Maathuis CGB. Ketenkwaliteit Cerebrale Parese Zorg. Een beschrijving van de cerebrale parese zorg en mogelijke verbeteringen hierin. April 2001, ISBN 90-75453-19-5

9. Nederhand MJ. Muscle activation patterns in post traumatic neck pain. March 2003, ISBN 90-75452-27-6

10. Jannink MJA. Usability of custom-made orthopaedic shoes in patients with degenererative disorders of the foot. September 2004, ISBN 90-75452-28-4

11. Blokhorst MGBG. State-dependent factors and attention in whiplash associated disorder. January 2005, ISBN 90-365-2111-4

12. Buurke JH. Walking after stroke co-ordination patterns \& functional recovery. Roessingh Research and Development, the Netherlands, February 2005, ISBN 90-365-140-8

13. van der Salm A. Spasticity reduction using electrical stimulation in the lower limb of spinal cord injury patients. Roessingh Research and Development, the Netherlands, October 2005, ISBN 90-365-2253-6

14. Snoek GJ. Patient preferences for reconstructive interventions of the upper limb in tetraplegia. Roessingh Research and Development, the Netherlands, December 2005, ISBN 90-365-2255-2

15. de Kroon JR. Therepeutic electrical stimulation of the upper extremity in stroke. Roessingh Research and Development, the Netherlands, December 2005, ISBN 90-365-2269-2

16. van Dijk H. Motor skill learning, age and augmented feedback. Roessingh Research and 
Development, the Netherlands, March 2006, ISBN 90-365-202-9

17. Mes CAJ. Improving non-optimal results in chronic pain treatment. Roessingh Research and Development, the Netherlands, January 2007, ISBN 90-365-2435-0

18. Voerman GE. Musculoskeletal neck-shoulder pain: a new ambulant myofeedback intervention approach. Roessingh Research and Development, the Netherlands, March 2007, ISBN 90-365-2460-1

19. Kallenberg LAC. Multi-channel array EMG in chronic neck-shoulder pain. Roessingh Research and Development, the Netherlands, March 2007, ISBN 90-365-2459-8

20. Huis in 't Veld MHA. Work-releated neck-shoulder pain: The role of cognitive-behavioural factors and remotely supervised treatment. Roessingh Research and Development, the Netherlands, December 2007, ISBN 978-90-365-2584-8

21. Fleuren JFM. Assessment of Spasiticity: From EMG to patients' perception. Roessingh Research and Development, the Netherlands, October 2009, ISBN 978-90-365-2869-6

22. Reenalda J. Dynamic sitting to prevent pressure ulcers in spinal cord injured. Roessingh Research and Development, the Netherlands, October 2009, ISBN 978-90-365-2884-9

23. Prange GB. Rehabilitation Robotics: Stimulating restoration of arm function after stroke. Roessingh Research and Development, the Netherlands, October 2009, ISBN 978-90-3652901-3

24. Vos-van der Hulst M. Prognostic factors and underlying mechanisms in chronic low back pain. Roessingh Research and Development, the Netherlands, February 2010, ISBN 97890-365-2881-8

25. Kottink-Hutten AIR. Assessment of a two-channel implantable peroneal nerve stimulator post-stroke. Roessingh Research and Development, the Netherlands, February 2010, ISBN 978-90-365-2959-4

26. van Weering MGH. Towards a new treatment for chronic low back pain patients. Roessingh Research and Development, the Netherlands, May 2011, ISBN 978-90-365-3305-8

27. Gulmans J. Crossing boundaries: improving communication in cerebral palsy care. Roessingh Research and Development, the Netherlands, February 2012, ISBN 978-90365-3305-8

28. Molier BI. Influence of augmented feedback on learning upper extremity tasks after stroke. Roessingh Research and Development, the Netherlands, March 2012, ISBN 97890-365-3293-9

29. Dubbeldam R. Towards a better understanding of foot and ankle kinematics in rheumatoid arthritis. Roessingh Research and Development, the Netherlands, October 2012, ISBN 978-90-365-3407-9

30. Evering RMH. Ambulatory feedback at daily physicial activity patterns. Roessingh Research and Development, the Netherlands, April 2013, ISBN 978-90-365-3512-0

31. Malhorta S. Does spasticity interfere with functional recovery after stroke? Roessingh Research and Development, the Netherlands, November 2013, ISBN 978-90-365-2567-0

32. Tabak M. Telemedicine for patients with COPD. New treatment approaches to improve daily activity behaviour. Roessingh Research and Development, the Netherlands, February 2014, ISBN 978-94-6108-590-0

33. Trompetter HR. ACT with pain. Measurement, efficacy and mechanisms of Acceptance and Commitment Therapy. Roessingh Research and Development, the Netherlands, September 2014, ISBN 978-90-365-3708-7

34. op den Akker H. Smart Tailoring of Real-Time physical activity coaching systems. Roessingh 
Research and Development, the Netherlands, October 2014, ISBN 978-90-365-3762-9

35. Jansen-Kosterink SM. The added value of telemedicine for physical rehabilitation. December 2014, ISBN 978-90-823196-0-6

36. Velstra IM. Advanced insights in upper limb function of individuals with cervical spinal cord injury. December 2015, ISBN 978-90-365-3929-6

37. Kloosterman MGM. Keep on rolling. Functional evaluation of power-assisted wheelchair use. June 2016, ISBN 978-90-365-4299-9

38. Prinsen EC. Adapting to change. Influenc of a microprocessor-controlled prosthetic knee on gait adaptations. December 2016, ISBN 978-90-365-4206-7

39. Wolvers MDJ. A coach in your pocket. On chronic cancer-related fatigue and physical behavior. March 2017, ISBN 978-90-365-4299-9

40. Cabrita M. Active and pleasant ageing supported by technology. November 2017, ISBN 978-90-365-4407-8

41. Haarman JAM. TIBAR - Therapist Inspired Balance Assisting Robot. November 2017, ISBN 978-90-365-4407-8

42. Nijenhuis SM. Roll up your sleeves! Technology-supported arm and hand training at home after stroke. April 2018, ISBN 978-90-365-4510-5

43. Cranen K. Acceptance of telerehabilitation in chronic pain: the patients' perspective. June 2018, ISBN 978-90-365-4555-6

44. Boerema ST. Sensing human activity to improve sedentary lifestyle. September 2018, ISBN 978-90-365-4604-1

45. Radder B. The wearable hand robot - supporting impaired hand function in activities of daily living and rehabilitation. November 2018, ISBN 978-90-365-4658-4

46. Krabben T. A reaching hand - towards an active therapeutic device for the upper extremity following stroke. December 2018, ISBN 978-90-365-4660-7

47. Timmerman JG. Cancer rehabilitation at home - the potential of telehealthcare to support functional recovery of lung cancer survivors. January 2019, ISBN 978-90-4701-7

48. Nikamp-Simons CDM. The sooner the better?! - Providing ankle-foot orthoses in the rehabilitation after stroke. May 2019, ISBN 978-90-365-4747-5

49. Achterkamp R. Towards a balanced and active lifestyle. June 2019, ISBN 978-94-6323656-0

50. Engbers C. Keep Cycling! - How technology can support safe and comfortable cycling for older adults. September 2019, ISBN 978-90-365-4848-9 



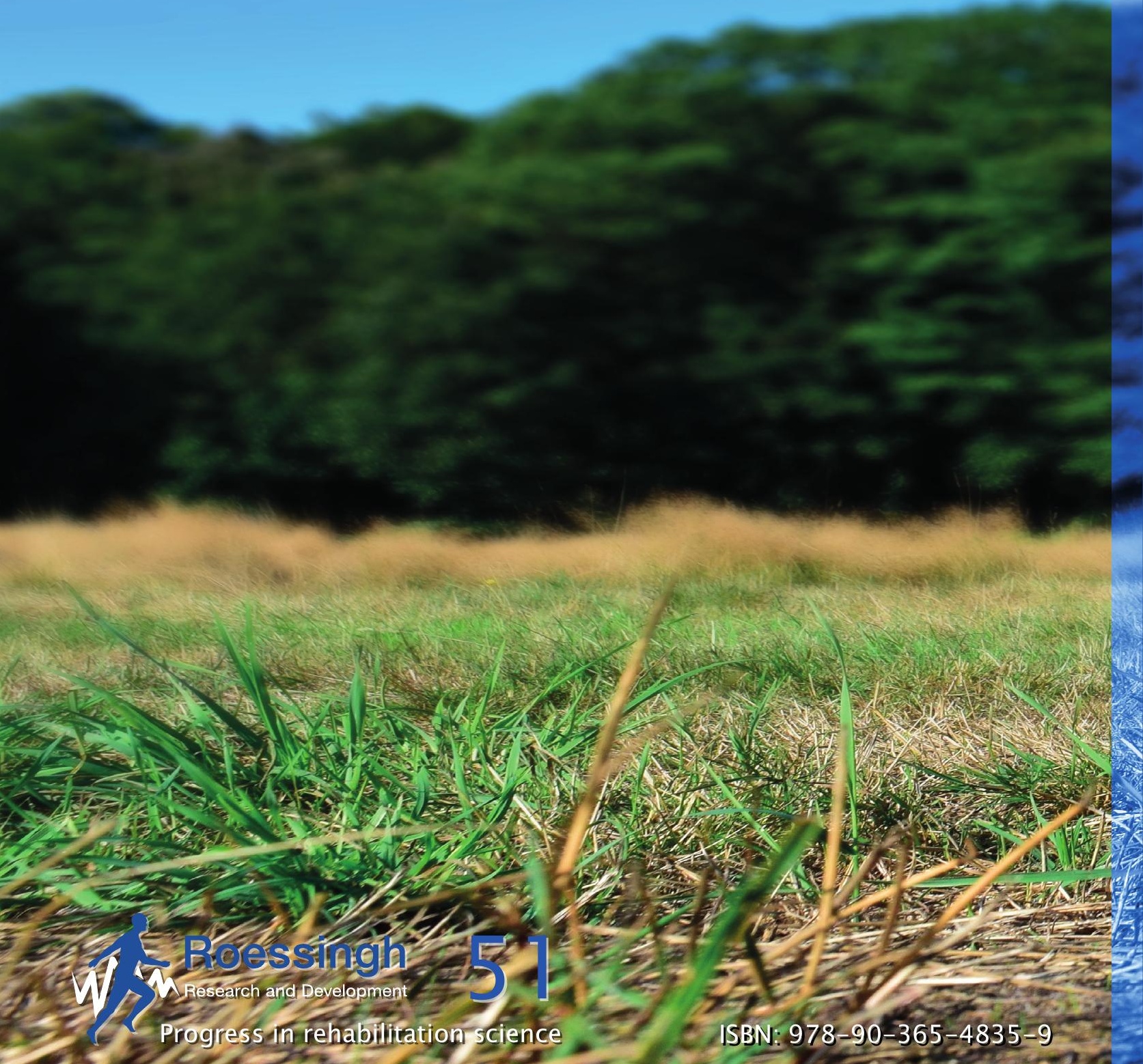